Portland State University

PDXScholar

Fall 12-1-2017

\title{
A Phenomenological Study of the Lived Experiences of Parents of Young Children with Autism Receiving Special Education Services
}

Donna Marie Barrow

Portland State University

Follow this and additional works at: https://pdxscholar.library.pdx.edu/open_access_etds

Part of the Special Education and Teaching Commons

Let us know how access to this document benefits you.

\section{Recommended Citation}

Barrow, Donna Marie, "A Phenomenological Study of the Lived Experiences of Parents of Young Children with Autism Receiving Special Education Services" (2017). Dissertations and Theses. Paper 4035. https://doi.org/10.15760/etd.5919

This Dissertation is brought to you for free and open access. It has been accepted for inclusion in Dissertations and Theses by an authorized administrator of PDXScholar. Please contact us if we can make this document more accessible: pdxscholar@pdx.edu. 
A Phenomenological Study of the Lived Experiences of Parents of Young Children with Autism Receiving Special Education Services

by

Donna Marie Barrow

A dissertation submitted in partial fulfillment of the requirements for the degree of

\author{
Doctor of Education \\ in \\ Educational Leadership: Special and Counselor Education
}
Dissertation Committee:
Randall L. De Pry, Chair
Ingrid Anderson
Christina Nicolaidis
Jana Meinhold

Portland State University

2017 
(C) 2017 Donna Marie Barrow 


\begin{abstract}
Over the past two decades reported rates of autism have steadily risen. The current incidence is 1 in 68 children. While autism can be reliably diagnosed at 18 months in most children with the condition, specialized autism treatment rarely begins before a child's third or fourth birthday. As screening and diagnosis procedures improve so does the need for effective early interventions for autism. Researchers and professionals have expressed a growing concern over the need for effective early interventions for infants and toddlers with autism. At the same time, there is a dearth of qualitative research exploring the needs and experiences of parents with a very young child with autism. Employing a phenomenological framework, the purpose of this study was to investigate the lived experiences of parents of a young child with autism receiving early special education services. Unstructured interviews and photo elicitation were used to generate rich, detailed descriptions of the phenomenon. Data analysis from photographic images and narrative dialogues illuminated six essential themes across participants: (a) parents as pioneers: forging the way for future families; (b) making the journey as a family; (c) navigating uncharted service systems; (d) overcoming challenges and obstacles; (e) resilience, ingenuity and hope; and (f) reflecting on the first three years and looking forward. Participants expressed that they felt this study gave them a "voice" in the research literature. This study is one of the first to investigate the lived experiences of parents as they seek and secure autism services for their child under five with autism.
\end{abstract}




\section{ACKNOWLEGEMENTS}

First and foremost I would like to thank the four participants who so generously shared their life experiences and knowledge with me. I would also like to thank my committee for their guidance and support of my research. Finally, I know all of this would not have been possible without the understanding, love, and encouragement from my husband, Ben and my daughter, Sophie. 


\section{TABLE OF CONTENTS}

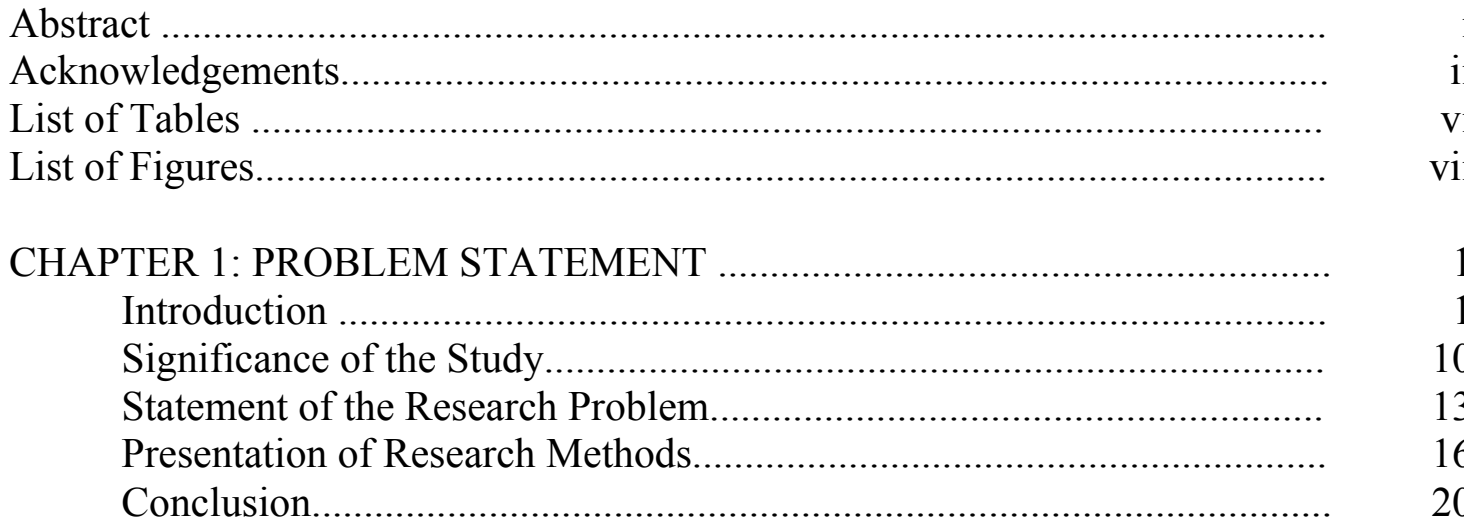

CHAPTER 2: LITERATURE REVIEW …………...................................... 21

Introduction.............................................................................. 21

Theoretical Frameworks..................................................................... 24

Phenomenology..................................................................... 24

Ecological Systems Theory........................................................ 26

Review of Research Literature on Early Interventions for Autism ........... 29

Review of Research Literature on Caring for an Infant with Autism ....... $\quad 55$

Review of Research Literature on Autism EI and IDEA, Part C .............. $\quad 66$

Review of Research Literature on Family Perspectives, Values,

Priorities \& Resources......................................................................... $\quad 72$

Review of the Methodological Literature................................................. $\quad 80$

Application of Research Literature to Present Study ................................ $\quad 89$

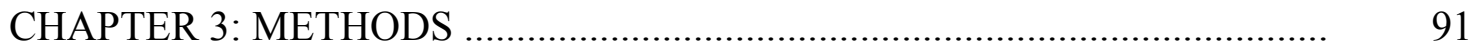

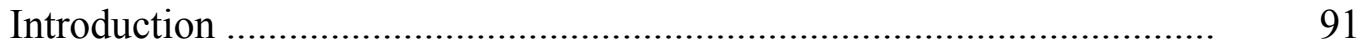

The Phenomenological Research Paradigm .............................................. 91

Research Questions ........................................................................... 95

Participant Recruitment ..................................................................... 95

Participant Selection Parameters …………………………........... 95

Snowball Sampling ……….......................................................... 97

Data Collection Methods ..................................................................... 101

First Interview Procedures ........................................................ 108

Second Interview Procedures ...................................................... 111

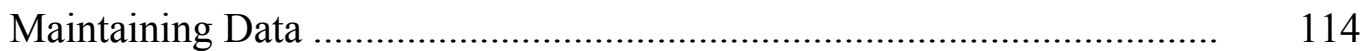

Role of the Researcher......................................................................... 115

Data Analysis............................................................................... 118

Addressing Research Validity.............................................................. 120

Chapter 3 Summary: ............................................................................ 123 
CHAPTER 4: RESEARCH FINDINGS AND DISCUSSION ........................ 124

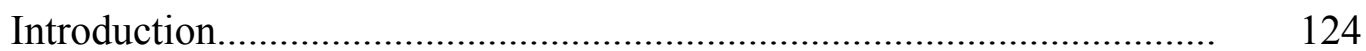

Section One: The Four Participant Narratives....................................... 131

Narrative 1: Caroline's Lived Experiences .................................. 132

Narrative 2: Emily's Lived Experiences .................................... 146

Narrative 3: Jessica's Lived Experiences ................................... 164

Narrative 4: Karen's Lived Experiences .................................. $\quad 180$

Section Two: The Essential Themes ................................................... 203

Essential Theme 1: Parents as Pioneers ...................................... 207

Essential Theme 2: Making the Journey as a Family ................... 212

Essential Theme 3: Navigating the Undeveloped New World

(Autism EI Service Systems) .................................................. 215

Essential Theme 4: Overcoming Obstacles and Challenges ........ 226

Essential Theme 5: Ingenuity, Resilience, and Perseverance ....... 232

Essential Theme 6: Reflecting on the First Three Years and

Looking Forward ................................................................ 235

Limitations ............................................................................... 237

Chapter 4 Summary ................................................................... 239

CHAPTER 5: REFLECTIONS, CONCLUSIONS AND IMPLICATIONS ....... 241

Introduction......................................................................................... 241

Section 1: How My Study Results Addressed the Research Questions

Research Question (1a): What are Parents Experiences of

Having a Young Child Recently Diagnosed with

Autism?.

Recognition of Early Markers and Facing Invalidation ....

248

Feelings about Accessing Services for Their Child ......... $\quad 252$

Navigating Services Birth to Age Three ......................... 254

Social Media Through Early Concerns and Diagnosis

Phases

Research Question (1b): What are the Structural Essences of

the Phenomena of Parenting a Very Young Child with Autism in the Context of EI Services?

Pioneering (Essential Theme One) ............................... 260

Making the Journey as a Family (Essential Theme Two). 261

Navigating the Undeveloped New World of Autism

Services for Very Young Children (Essential Theme

Three)

Overcoming Obstacles and Challenges (Essential Theme

Four).

Ingenuity, Resilience, and Perseverance (Essential

Theme Five)..

Reflecting on the First Three Years and Looking

Forward (Essential Theme Six) 
Section Two: Implications, Future Directions and Limitations

The Study's Findings in the Context of Bronfenbrenner's

Ecological Systems Theory

Testing Hypotheses Generated from the Results of this Study..

Replication with Other Populations

Appendix A: Recruitment Materials for Snowball Sample

Appendix B: Recruitment Email [to participant]

Appendix C: Recruitment Flyer [to participant].................................................... 308

Appendix D: Initial Phone Script ………………............................................ 309

Appendix E: Resource List ..................................................................... 312

Appendix F: First Interview Script \& Protocol .............................................. 315

Appendix G: Informed Consent \& Child Assent .............................................. 317

Appendix H: Second Interview Script \& Protocol ............................................ 323

Appendix I: Artifact Catalogue ……………………...................................... 325

Appendix J: Audit Trail and Auditor Attestation ............................................. 326 


\section{LIST OF TABLES}

Table $1 \quad$ Rhetoric Describing ABA Practices................................................. 49

Table $2 \quad$ Participant Reported Response from Pediatrician .......................... 209

Table $3 \quad$ Participants Concerns, Wait times, Services (in months) ............... 216

Table $4 \quad$ Services Participants Were Receiving at the Time of the Study.... 218

Table $5 \quad$ Participant Activities to Support Child Engagement ...................... 234

Table $6 \quad$ Essential Themes and Subsidiary Themes ....................................... 247

Table $7 \quad$ Participants' Report of Child's Earliest Manifestations of Autism 249

Table $8 \quad$ Recommended Service Levels for Oregon Children ....................... 265 


\section{LIST OF FIGURES}

Figure 1 Organizing Principle for Literature Review..................................... 21

Figure 2 Bronfenbrenner's Ecological Systems Theory................................ 27

Figure $3 \quad$ Family Systems Intervention Model.............................................. 29

Figure 4 Themes in ASD-EI Literature...................................................... 80

Figure $5 \quad$ Photograph C-2: Caroline's family .............................................. 135

Figure 6 Photograph C-3. Caroline's son Nathan and his infant brother....... 136

Figure $7 \quad$ Photograph C-5 Caroline's son after ear surgery ........................... 140

Figure $8 \quad$ Photograph C-7 Nathan with his occupational therapist ................ 145

Figure 9 Photograph E-1 Emily's children with their aunt ........................... 147

Figure 10 Photograph E-2 Emily's children in a hotel while on vacation ...... 148

Figure 11 Photograph E- Emily's husband standing in the driveway ............. 149

Figure 12 Photograph E-4 Emily's children on Christmas morning .............. 151

Figure 13 Photograph E-5 Emily's children with their grandmother ............. 152

Figure 14 Photograph K-1 Karen, her husband, and Carter ............................ 182

Figure 15 Photograph K-2 Carter's $3^{\text {rd }}$ birthday ............................................. 183

Figure 16 Photograph K-3 A family beach day ............................................. 187

Figure 17 Box display of comparison between photographs K-4 and K-5 ..... 189

Figure 18 Photograph K-4. Karen is holding Carter at 12 months ................. 196

Figure 19 Box display of Karen's last five photographs ................................ 201

Figure 20 Box display of email from Karen .................................................. 202

Figure 21 Graphic representation of participants' journeys ............................ 206 


\section{CHAPTER 1 \\ PROBLEM STATEMENT}

\section{Introduction}

This dissertation was a phenomenological study that investigated the lived experiences of parents with a young child with autism and their experiences with special education early intervention services (EI). Phenomenological research methodology was used to capture the lived experiences of the participants and the essence of the phenomenon under investigation (Husserl, 1970). By doing so, the research findings of this study offer insight into parents' perspectives about EI for their very young child with autism. This outcome is particularly important because the field of autism early intervention (AUT-EI) is an emergent area of study and practice (Camarata, 2014; Zwaigenbaum, 2015) and few qualitative studies have investigated parenting and this context (Grindle, Kovshoff, Hastings, \& Remington, 2009). This study utilized two theoretical frameworks (a) phenomenology and (b) Ecological Systems Theory (ECST). The phenomenological framework allowed me to analyze relevant literature from various fields of study and engage in reflective research activities. The ECST lens allowed exploration and analysis of the many ecological contexts influencing the experiences of parents with a young child receiving autism related services. These ecologies included family life, early intervention, and medical services.

There is little research related to the lived experiences of parenting young children (i.e., under age 5) with autism. Early intervention models for children under 3 with autism are in the research / pilot stages of development (see Rogers \& Vismara, 2014). Early intervention services are available for the small percentage of infants and toddlers 
diagnosed with autism, however family-centered EI are most often individualized intervention plans and not specialized AUT-EI models for emergent autism (Stahmer \& Mandell, 2007). While markers of autism are evident around a child's first birthday, the research indicated that children with autism do not receive specialized treatment until after age 3 (Baio, 2012; Elsabbagh \& Johnson, 2007). Identifying AUT-EI was described as a high priority in the field by predominant researchers (Rogers \& Vismara, 2010; Zwaigenbaum et al., 2015).

Very few studies have explored the unique lived experiences of parents of young children with autism as they navigated the educational service systems. This study illuminated the lived experiences of parents of children under age 5 with autism. The results provided insight into the phenomena of parenting and experiences with EI and early childhood special education (ECSE). The dissertation was conducted using two data collection methods (a) unstructured interviewing (Vagle, 2014) and (b) photo-elicitation interviewing (Harper, 2002) with four mothers of young children with autism. The children's mean age was 42 months. All four mothers were married. All participants were recruited from a large metropolitan region in the Pacific Northwest. I conducted two interviews with each participant. In order to capture one participant's full lived experiences I added a third interview with that participant. The phenomenological paradigm is a flexible, reflective, and iterative method for exploring lived experience (Vagle, 2014). As a result, the study was amended to add a group universal member check meeting after the completion of data collection. This group process served to 
confirm participant acceptability of the essential themes that emerged from the narrative and photographic data.

The participants and I met individually for the first interview. First interviews lasted between 1 to 2 hours. I had instructed each parent to collect 10 family photographs prior to our meeting. I structured the first meeting using the photographs and also with written prompts. The intent of the first meeting was to establish a trusting rapport and begin exploring each parent's lived experiences. Consistent with the photo-elicitation research, the parents in this study used the images as a tool as they reflected about their family lives and experiences with their child. The first interviews generated fine-grained, qualitative data (Cridland, Jones, Caputi, \& Magee, 2014).

The participants and I met individually for the second interviews. I reviewed our conversation from the first interview and gave the participants an opportunity to return to the photographs and elaborate. I again used prompts that I developed based on first interview conversations. During the second interview, I also engaged with the participants in discussions about services and interventions. Interestingly, service experiences dominated most of both interviews. As one participant noted "you cannot separate services from parenting. It's the ever-present elephant in the room with autistic kids."1

\footnotetext{
${ }^{1}$ Some researchers including authors with autism prefer "autistic" over the person-first convention "person with autism." In accordance with APA and the precedent in the field of special education research and practice I use person-first language in this dissertation. However, participants in this study sometimes used "autistic" when referring to autism characteristics.
} 
The conversations with each parent flowed naturally and generated over 300 pages of transcripts from which the essential themes emerged. The methods and procedures for these research activities will be discussed in detail in Chapter 3 .

The purpose of this chapter is to provide a background on emergent autism, early intervention services for young children with autism, and parenting experiences with children with autism between three and five years old. This chapter will also present the research problem, significance, the study's research questions, and data collection methods. Chapter 1 will conclude with a discussion of the study's limitations.

\section{Background}

Characteristics of autism. Autism is a neurodevelopmental condition affecting 1 in 68 children in the United States (CDC, 2014). Autism is characterized by qualitative impairments in social and communication behavior and restricted interests/ repetitive behaviors. Autism is lifelong condition with an age of onset sometime after 12 months (Dawson, 2008). Autism is heterogeneous and, as a result, symptom presentation can vary across several core areas of development (i.e., social, language, patterns of interests) (American Psychological Association [APA], 2013). Until recently autism was considered a spectrum of disorders. The most recent diagnostic criteria classified autism as one heterogeneous condition (APA, 2013). The core features of autism are marked impairments in social communication and markedly restricted repertoire of activity and interests (APA, 2013). Although to date there is no reliable index of cognitive global functioning for individuals with autism (Rao, Raman, Thomas, \& Ashok, 2015), a number of studies found subgroups of children with autism ranging from significant to 
minimal impairments (e.g., Flanagan et al., 2015; Kim, Macari, Koller, \& Chawarska, 2015). To date there is no known medical screening for autism and no known cure. However, there have been recent advancements in the areas of physiology, neurology and genetics that point to underlying neurodevelopmental causes (Courchesne, Campbell, \& Solso, 2011; Dawson, 2008; Elsabbagh \& Johnson, 2010).

Characteristics of autism are also described as strengths. Prizant (2015) advocated for intervention strategies that view some characteristics of autism as coping strategies in a world that feels chaotic and overwhelming. Prizant described this new conception of autism as shift away from the long-standing deficit view whereby autism characteristics are seen as developmental problems to be corrected. Instead, Prizant promoted building on a child's individual strengths, including features of autism, as part of the intervention process. This burgeoning ideological shift in the mainstream literature is a relatively new way of conceptualizing the characteristics associated with autism. Disability rights advocates and researchers preceded this shifting paradigm. They are vocal objectors of a deficit view of autism (Robertson, 2010). There is increasing number of articles from the neurodiversity framework for understanding autism in the disability rights literature (e.g., Fenton \& Krahn, 2009; Robertson, 2010). This area of research acknowledges the strengths, gifts, and talents of people with autism living in a world socially designed for people without autism (ASAN, 2009; Prizant, 2015; Robertson, 2010).

Emergence of autism. Since the 1980s, there has been considerable research on the symptoms of autism in infants and toddlers (ages 0 to 3 ). Seminal retrospective studies including first birthday home-movie analysis, as well as parent reporting, were the 
first to identify differences in babies that later went on to receive a diagnosis of autism (e.g., Adrien et al., 1993; Baranek, 1999; Osterling \& Dawson, 1994; Osterling, Dawson \& Munson, 2002).

Over the last two decades, researchers turned their attention to the emergence of autism's markers in infants with an older sibling already diagnosed with autism (INFSIBS), the only identified high-risk group (e.g., Landa, Garrett \& Mayer, 2006; Zwaigenbaum et al., 2005). Infants with an older sibling already diagnosed with autism have close to a $20 \%$ chance of developing autism (Elsabbagh, \& Johnson, 2010; Ozonoff, 2011). Prospective INF-SIBS research confirmed early markers of autism and the atypical developmental trajectory in INF-SIBS.

As a result of national autism screening initiatives (Johnson \& Myers, 2007) and assessments validated in toddlers as young as 18 months (i.e., Autism Diagnostic Observation Schedule (ADOS), Lord, Rutter, DiLavore, Risi, 1999), the number of infants / toddlers with autism in need of effective interventions is increasing and projected to continue (Rogers \& Vismara, 2014). Only one first-generation autism-EI model (i.e. Wetherby, et al., 2014) is congruent with federal requirements and best practices in EI services (DEC, 2014; IDEA, 2004; Keilty, 2010). This congruence involves family support planning as well as intervention strategies targeting core symptoms of autism. Interventions for autism will be discussed later in this section along with a critique of clinical assumptions about the role of AUT-EI in improving outcomes early in the trajectory or even ameliorating core symptoms of autism altogether. 
Treatments for children with autism. Autism treatment models for older toddlers and preschool age children (i.e., 36 to 60 months) are available and evidencebased. Research indicates these treatments positively affect developmental outcomes in children with autism as young as 18 months, particularly in social-communication (e.g., Rogers \& Dawson, 2010 ; Prizant, Wetherby, Rubin, \& Laurent, 2003). Comprehensive treatment models for young children with autism are typically clinic- or school-based and often delivered by a clinician or teacher. These curricula are based on the principles of applied behavior analysis (ABA) within a developmental framework. Several models for

young children are widely available and have a strong evidence base. The following list of models is not exhausted but an examples of those with an evidence base: Early Start Denver Model (ESDM; Rogers and Dawson 2010), Reciprocal Imitation Training (RIT; Ingersoll and Schreibman 2006), and Social Communication/Emotional Regulation/ Transactional Support (SCERTS; Prizant et al., 2003)

\section{Early Intervention Models for infants and toddlers with autism. First-} generation AUT-EI models (i.e., birth to 36 months) are still in the pilot phase and are not available outside of the research context. Recently, specialized comprehensive models more appropriate for infants and toddlers were developed and began reporting on pilot data. First-generation AUT-EI comprehensive treatments addressed emergent autism symptoms, involved parents in the implementation, and many were delivered in natural family settings (Rogers \& Vismara, 2014; Zwaigenbaum et al., 2015). Parent involvement and intervention in embedding goals in natural family routines are distinguishing features of AUT-EI. 
Treatment efficacy is one priority in designing interventions for infants and toddlers with autism (Rogers \& Vismara, 2014). Another is congruence between specialized autism EI models and the Individuals with Disabilities Education Improvement Act, Part C ([IDEA, Part C], IDEA, 2004). IDEA Part C is the federal regulation for all EI services for infants and toddlers with special needs from birth through age 2. IDEA, Part $\mathrm{C}$ requirements incorporate practices with demonstrated efficacy with parents and young children with other disabilities (Copple \& Bredekampm, 2009; DEC, 2014). Research has found that interventions for infants and toddlers are most effective when (a) parents are equal partners in the EI process, (b) family priorities are considered in planning, (b) and interventions are implemented at home by parents within family home routines (Bruder, 2000; Dunst, 2000; Farell, 2009; Kalyanpur, Harry, \& Skrtic, 2000). The small number of first generation AUT-EI model did not describe treatment components consistent with family-centered EI procedures (Rogers \& Vismara, 2014; Zwaigenbaum et al., 2015).

Some research teams expressed concern over the lack of congruence between first generation AUT-EI models and IDEA, Part C (Camarata, 2014; Dawson, 2008; Rogers Zwaigenbaum et al., 2015). Some of the AUT-EI models involved parents in the treatment, but in all cases, the parents implemented strategies based on child-centered curricula and did not participate in collaboration activities with interventionists to develop a family plan to meet child goals (e.g., Rogers et al., 2012; Schertz, Odom, Baggett \& Sideris, 2013; Wetherby et al., 2014). Piloted first-generation AUT-EI treatments were situated in family routines, however, without collaborative information 
gathering about family priorities, resources and goals, the natural contexts within which interventions were situated may not have reflected routines preferred by parents (McWilliam, Casey \& Sims, 2009). Upon analyses of AUT-EI study methodologies and operationalized procedures for this proposal, none of the AUT-EI studies found in the literature reported parent collaboration activities in a manner consistent with EI for all eligible infants and toddlers (see Woods \& Brown, 2011).

Parenting and service experiences with a young child with autism. The quality of relationships and attachment between a parent and a young child with autism is as strong as with other populations of parents / young children (Buerkens, Hobson, \& Hobson, 2013; Siller \& Sigman, 2002). In some ways, parenting a child with autism adds uniquely positive experiences to family life (Hunt-Jackson, 2007; Lutz, Patterson, \& Klein, 2012). At the same time parenting a child with autism is associated with increased stress. More information is needed about the lived experiences of parents with infants with autism receiving EI. There is evidence that parenting a child with autism is associated with greater stress than parenting children with developmental delay (DD) or typical development (TD) (Estes et al., 2009; Griffith et al., 2010). Some of the reported sources of stress included (a) severity of child's autism symptoms (Stuart \& McGrew, 2009), (b) infant's ability to self regulate (Davis \& Carter, 2008), and (c) feelings of stigmatization and lack of support (Osborne, McHugh, Saunders, and Reed, 2008). Qualitative investigations of parent perspectives suggested that there is increased stress related to the amount of services involved in treating autism and the ways information about autism are provided (Grindle et al., 2009; Patterson \& Smith, 2011; Webster et al., 
2004). Relationships with service providers also contributed to parent satisfaction and stress (Coogle, 2013). Applequist and Baily (2000) found parents showed a strong desire for involvement in intervention and felt uncertainty in their role in developing EI plans for their child. Parents of children with autism desired social networks and relationships with other families with young children with autism (Grindle et al., 2009).

There were few studies directly investigating experiences of parents with a young child with autism as they navigate the EI system. This parenting experience may be very different than that of parents with young children with other types of disability.

\section{Significance of the Study}

Children with disabilities benefit more from interventions during the first three years of life than any other time in development (Dawson, 2008; Elsabbagh \& Johnson, 2010) because of early brain plasticity and the rapid physiological changes (Courchense, Campbell \& Solso, 2011). Despite recent efforts to identify and treat autism in the first few years of life, most infants and toddlers with autism remain undiagnosed for years and do not receive autism-focused treatment until after their third birthday (Shattuck et al., 2009; Zablotsky et al., 2017). Zablotsky and colleagues (2017) reported that heterogeneity of early autism influences the age of diagnoses. Only one in five children with autism are diagnosed before their third birthday (Zablotsky et al., 2017). Some very young children later diagnosed with autism received EI services for developmental delay or speech and language service (Stahmer \& Mandell, 2007). There is a two-year lag time between emergence of autism markers and specialized treatment. Most infants and toddlers with autism are overlooked and do not receive family supports afforded to many 
other infants and toddlers with disabilities. Federal legislation guarantees the right to EI for infants and toddlers with or at risk of a disability, as identified in IDEA, Part C (2004).

Nearly all AUT-EI research teams expressed that identifying effective AUT-EI is a high priority (Camarata, 2014; Dawson, 2008; Elsabbagh \& Johnson, 2007; Rogers \& Dawson, 2010; Zwaigenbaum et al., 2015). However, effective treatment for autism in children under three have not been established. The current AUT-EI models focused on identifying treatments that increase social-communication as early as possible infant with autism. First generation AUT-EI models incorporated principles of applied behavior analysis (ABA), naturalistic strategies, and developmental models. Some of the predominant research groups described first-generation AUT-EI models as "scaled down," adapted models that were designed for older children with autism (Rogers \& Vismara, 2014; Zwaigenbaum, 2015). Many first generation AUT-EI models included collaboration with families to identify the best routines and gather information pertinent to implementation of specialized curricula. However, the AUT-EI intervention studies lacked information on family social, racial, and economic demographics. The models also did not include methodology to gather information on family resources, priorities, and culture. The Federal requirements under IDEA, Part C (2004) requires gathering of these family data. Family involvement is the cornerstone of both research-based practices and federal EI service requirements under Part C (Keilty, 2010). Without congruence with Part C, even effective AUT-EI treatments would not likely be accessible to most families with an infant / toddler with autism. AUT-EI research teams are seeking to remedy this 
problem and align treatment models with Part C (e.g., Vismara et al., 2009; Wetherby et al., 2014).

Researchers still know little about service needs of parents with young children with autism (Coogle, Guerette \& Hanline, 2013; Karst, Vaughn, Van Hecks, 2012). In a recent review, Reed and Osborne (2014) described the AUT-EI research on parent perspectives as poorly documented. Qualitative caregiving studies with older children with autism were also limited but indicated that a number of autism-related and sociocultural factors shaped parent perspectives on services and daily, lived experiences. Contemporary child development theories emphasized that development is contextualized and influenced by biological, proximal, and distal influences (Brooks-Gunn, 1995). Early intervention philosophy for all families with very young children with disabilities takes these factors into account when developing family early intervention plans (Bruder, 2000; Dunst \& Trivette, 2009; IDEA, 2004). In response to the lack of parent perspective research, Karst, Vaugh and Van Hecks (2012) recommended an evaluation protocol for proposed AUT-EI models that incorporates effects on both parent (e.g., self-efficacy, stress, mental health) and child outcomes.

Over the past two decades, reported rates of autism have steadily risen. The current incidence is 1 in 68 children, and 1 in 42 boys (Centers for Disease Control and Prevention [CDC], 2014). Some studies reported that the U.S. the prevalence of autism increased by $119.4 \%$ between 2000 and 2010, making autism the fastest growing disability (CDC, 2014). It is still unclear whether this increase in autism is a result of improved diagnostic practices or if it is, in fact, an unusual epidemiological phenomenon. 
Either way it is expected that more infants and toddlers with autism will be entering the EI system. This underscores the need for AUT-EI that is acceptable to parents.

This study investigated the unique experiences of parents with a very young with autism and EI services. I initially intended to limit the participants to parents with infants and toddlers (i.e., birth through 2) with autism. However, early in the recruitment process it was became evident that there were few or no families meeting the recruitment criteria because children were not receiving autism treatment until close to their third birthday. As a result, I expanded the child's age parameter to age five.

The results of this study provided insight into contextual influences on parenting a child with autism and the meaning parents make of their experience in that context. The lived experiences of the parents in this study and the essential themes add a new understanding of service needs for parents of young children with autism. Recently, some autism research groups voiced a need for this type of qualitative investigation into parenting young children with autism (Bolte, 2014; Cridland et al., 2014).

\section{Statement of the Research Problem}

This study sought to give voice to parents of very young children with autism. The first three years from diagnosis to autism services represents a time when family experiences are largely unexamined. This is an important research problem to address for several reasons. First the literature indicates a two-year lag time between when parents of children with autism first notice concerns over their child's development and initiation of services. Lived experiences during this lag time are absent from the literature. However, rich descriptions of these experiences may add insight into treatment needs of very young 
children with autism and their families. Secondly, hearing from parents in their own words about their lived-experiences during this time may inform services and also advocacy efforts. Thirdly, the participants' experiences have the potential to touch the lives of other families and those who work with them, countering the effects of isolation and discouragement. The remainder of this section focuses on the gaps in qualitative research in this area and explains the research problem in greater detail.

Phenomenological investigation was appropriate to illustrate a deeper meaning of parenting a very young child with autism and the role of EI in family life. As a methodological approach, phenomenology allows for examination of parental lived experiences associated with many ecological influences (e.g., family culture, personal history and societal views of autism). There is growing interest in qualitative investigation in the field of autism (Bolte, 2014; Cridland et al., 2014). There is also an increasing acknowledgement of a need for multiple research methodologies in special education (Odom, Brantlinger, Gersten, Horner, Thompson, \& Harris (2005).

I found no phenomenological investigation of caregiving an infant / toddler with autism in the research literature. There is also a dearth of qualitative investigation of experiences parenting an infant or toddler with autism and receiving EI. There is a need to address these gaps. Extending qualitative research to gain insight into parents' lived experiences during the EI process may shed light on this unique parenting context. This aim is particularly important in the field of EI because the most effective interventions for infants and toddlers with other disabilities involve parents acting as equal partners in intervention. A strong collaborative relationship between parent and provider is a 
prerequisite for effective treatments, delivered in the natural family environments (DEC, 2014; Dunst \& Trivette, 1994; Dunst \& Trivette, 2009; IDEA, 2004; Keilty, 2010). Autism intervention has a tradition of child-focused, clinician-implemented, specialized, and intensive intervention practices (National Research Council [NRC], 2001).

The marginal role of qualitative investigation found in the AUT-EI literature may have implications for the quality of life for parents, their child with autism, and other family members (Reed \& Osborne, 2014). Investigations into parental lived experiences may illuminate effects of early autism and EI services on parenting in a way in which quantitative investigations cannot. An understanding of what parenting an infant with autism means and what experiences with EI means, would be difficult to capture in quantitative investigation. Phenomenological research may provide nuanced insight into the effects of emergent autism, the emotional impacts on family, and the joys and hardships.

In the field of EI for all infants and toddlers, child outcomes are associated with the role parents play in their children's treatment (Ketelaar, Vermeek \& Hart, 1998; Skonkoff \& Hauser-Cram, 1987). In these other EI populations, links between familycentered practices and later outcomes included child development scores (Mahoney, Boyce, Fewell, Spiker, \& Wheed, 1998), parental sense of efficacy, and effectiveness in implementing interventions (Peterson, Luze, Eshbaugh, Jeon \& Ross-Kanz, 2007; Trivette, Dunst \& Hamby, 2010). 


\section{Presentation of Research Methods}

Using photo-elicitation (Collier, 1957; Harper 2002) and unstructured interview methodology (Vangle, 2014), I explored the lived experiences of parents of young children with autism. I had proposed selecting participants with a very young child diagnosed with autism (24 to 36 months). However, it became clear during the recruitment process that due to this study's research problem (i.e., lack of early intervention services for infants and toddlers with autism) I had to extend the recruitment criteria to include children up to age 5 with an autism diagnosis and receiving special education services. The children in this study were between four and five years old. The mean age parents sought help from pediatricians about their children's development was 12 months. The mean age of autism diagnosis was 29 months.

The purpose of this study was to gain insight into the lived experiences of parents of infants and toddlers with autism. This involved more than documenting the parent's perspective, although this played a role. The aim of this study was to capture the essence and nuances (Van Manen, 1997) of life as a parent of a young child with autism.

The following were the research questions for this dissertation:

1. What are the participants' lived experiences as parents with a young child (under age five) with ASD as they relate to early intervention services?

1a. What are the participants' experiences of having a young child diagnosed with ASD within the past two years?

$1 b$. What are the structural essences of the phenomena of parenting a very young child with ASD in the context of EI services?'

I employed qualitative, phenomenological methodology (Vagle, 2014; Van Manen, 1997) to investigate my research questions. Phenomenological methodology was well suited to explore the meaning parent participants make of their experiences. 
Phenomenology emphasizes investigation of the manner in which individuals construct their lives and make meaning of their experiences (Cridland et al., 2014; Meadan, Stoner \& Angel, 2010). This study revealed essential themes associated with the lived experiences of parenting a young child with autism and navigating early service systems.

There are many qualitative research traditions. Examples include: case study (Stake, 1995), ethnography (Weisner, 1996), and grounded theory (Strauss \& Corbin, 1994). Phenomenology is an interpretive, open, flexible and responsive method allowing the researcher to deeply and thoughtfully settle into and sit with the phenomenon under investigation (Vagle, 2014). Van Manen (1997) described this process as reflective, one in which the researcher deeply contemplates the concrete ways in which phenomena are lived. One's lived experience is a philosophical construct that delineates meaning independent of personal preconceptions and bias (Vagle, 2014).

\section{Research Activities}

Screening and Interviews. Prior to scheduling the interview I conducted 15minute phone screenings with seven parents. Two parents opted out of the study and one did not meet the inclusion criteria. Four participants met the inclusion criteria and participated in the interviews and the group universal member check meeting.

I had proposed two interviews with each parent participant and conducted two interviews with three of the parents. One participant expressed a desire for a third interview because she felt she had more to share. Prior to the first interview, I asked the parent to gather a set of 10 family photographs and explained how the photographs would be used during the interview process. Both interviews were structured using specific 
prompts related to the phenomenon and research questions. The first interview was guided by a discussion of the pictures. First interview data (i.e., narrative interview transcripts and photographic data) were analyzed and used to prepare for the second participant interview. During the second unstructured interview, we reviewed the first interview conversation and kept the photographs available to reference if needed. After reviewing notes from the first interview as a member checking procedure, the participant and I engaged in a deeper conversation about navigating the service system from diagnosis to treatment. During both interviews service experiences emerged as an essential theme. Throughout the interview process I used prompting strategies to explore topics that addressed the study's research questions. I audio recorded the interviews, transcribed, coded, and implemented three member-checking strategies to insure I accurately captured the participants' lived experiences and to neutralize my own biases and preconceptions. I maintained a flexible researcher stance (Grafanaki, 1996) and as the research study progressed, the participants and I realized the data gathering process needed to be expanded to include a group member check meeting so the participants had a chance to confirm the essential themes and study's conclusions. I amended and resubmitted the Institutional Board Review (IRB) human subjects application. Portland State University's IRB committee approved the proposed changes.

\section{Data analysis.}

Essential themes emerging from the data. Given that the goal of the present study was to understand more about the lived experiences and perspectives of parents and their daily life with their children (i.e., parenting and early intervention experiences), the 
phenomenological approach was very effective. Each parent presented a unique personal experience imbued with perceptions about self, their child, treatment, and ecological systems influencing family life. In-depth conversations yielded the seven essential themes shared by all participants.

- Parent's as pioneers. Parents forged the way for other families with infants and toddlers with emergent autism. Because parents encountered little awareness of early manifestations of autism, they experienced invalidation. All four parents continued to seek answers. They shared their experiences with other parents through social media groups and connection with other families with children with autism.

- Navigating the undeveloped world of autism services. While the parents in this study received early intervention services approximately a year after their first concerns for their children's development, they did not receive specialized autism services until their children were close to age 3 ( $\mathrm{m}=32.5$ months). The lag time between first concerns ( 9 months) and commencement of specialized autism services represents a journey into unchartered territory.

- Overcoming challenges and obstacles. During the first three years of their children's lives parents in this study faced and overcame extremely difficult obstacles. Stress and worry over children's undiagnosed developmental problems was one example. Lack of sleep, children's health problems, and unexpected interruptions were common lived experiences across parents.

- Resilience, ingenuity, and hope. All of the parents in this study withstood uncertainty in getting help for their child. They used ingenuity in piecemealing services across systems. Ingenuity also took the form of accommodations and advocacy for their children's' autism. Finally, there were moments of hope that acted as inspiration or beacons that helped them navigate their way.

- Making the journey as a family. Family stood out as an essential theme. The immediate family members related to each other and were bonded by family culture. Participants described family traits, likeness, and belonging among all members including their children with autism. Extended family also played a role during the journey to diagnosis and autism treatment. Parents advocated to extended family members. The participants expressed respect, understanding, and patience when addressing extended family members' doubts about their children's autism diagnoses.

- Reflecting on the first three years and looking forward. The parents in this study arrived in their new life when their children were receiving services and participating in typical childhood experiences. Parents had learned to navigate the systems, help their children engage in the world, and advocate to the community. Parents had forged a trail for future families and shared their knowledge through social media autism parenting groups. 
The findings of this study contextualized the complexity of family life and its impact on parenting, as well as the unique experience of parenting a young child with autism who is receiving EI services.

\section{Conclusions}

In this chapter, I introduced my research study. This study addressed the need for understanding of parents' lived experiences during the lag time between first concerns and autism services. The intent of the study was to gather rich, detailed information about everyday lives of participants in order to give them a voice in the research literature and for their voice to reach other families and providers. In doing so the findings of this study may inform parent support initiatives, AUT-EI development, and advocacy. In telling their stories the participants themselves may have felt validated.

In Chapter 2 I will present the theoretical frameworks that guided the study. Specifically, I discuss the ecological systems theory and phenomenology as they pertain to the lived experiences of parents with an infant / toddler with autism receiving EI. I will also explore the AUT-EI research literature in Chapter 2. Phenomenology guided my research and effectively isolated the phenomena through every phase of the process. Because phenomenology requires a researcher's continuous, reflective engagement with data and analysis, I isolated the phenomena and continually reflected on my own biases and preconceptions. The ecological systems theory allowed me to maintain a contextualized view of autism research and also informed the study's activities through acknowledgement of bidirectional influences of ecologies that influence parenting and child development (Bronfenbrenner, 1978). 


\section{CHAPTER 2 \\ LITERATURE REVIEW}

"Variations in developmental processes and outcomes are considered a joint function of the environment and of a developing person." Bronfenbrenner (1993)

\section{Introduction}

This chapter presents the literature on parenting a young child with autism receiving EI and Early Childhood Special Education (ECSE) services. To understand participants' experiences as parents and recipients of early special education services, I reviewed both the parenting and the intervention literature. Four major themes emerged from the literature: (a) early intervention and autism, (b) caring for an infant with autism, (c) autism and the Individuals with Disabilities Education Act, Part C (IDEA, Part C), and (d) parent perspectives. These four prominent bodies of research intersected and, as a whole, represent the most salient research findings about parents with a very young child with autism. This literature review is organized around these four themes. Figure 1 illustrates the organizing principle for this chapter.

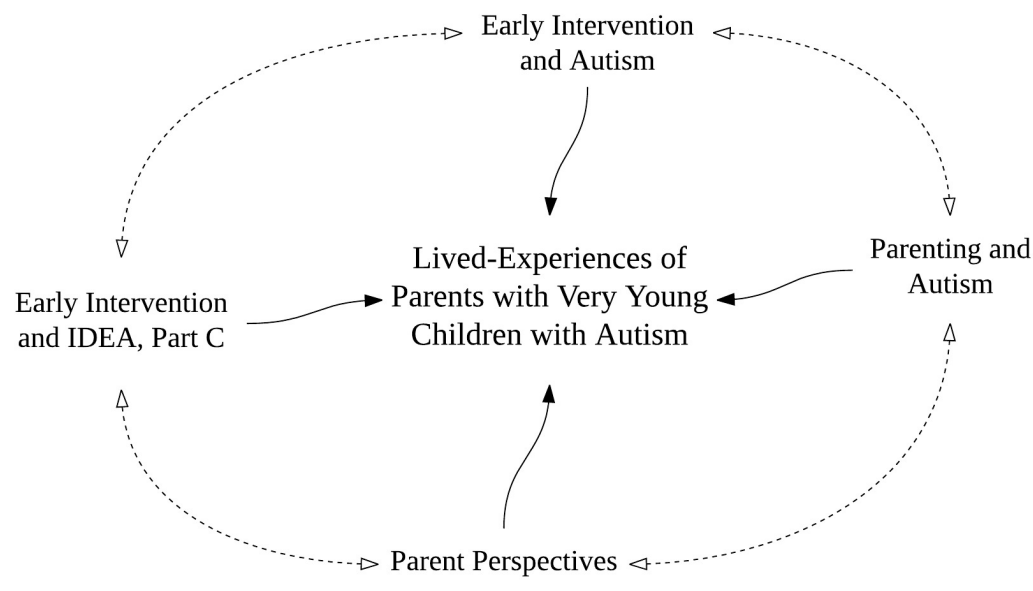

Figure 1. Organizing principle for review of AUT-EI research literature. 
Theme 1: Early intervention and autism. Early intervention and autism (AUTEI) was a prominent theme in the research literature. I present this area of research first because it serves to define autism services in the context of the larger field of early intervention (EI). As I reviewed the literature on autism and young children, I found that nearly all research teams in the field emphasized the need for early identification of autism and effective interventions as early as possible. Interventions studies focusing on emergent autism (i.e., birth to 3 ) and the need for better understanding of how to treat autism in the first few years of life stood out in the research. I also found that most of the first-generation early intervention models (AUT-EI) were not congruent with familycentered models for young children with other disabilities.

Theme 2: Caring for an infant with autism. The second theme, Caring for an Infant with Autism, was selected for its relevance to the lived experiences of parenting an infant/toddler with autism. In the broader EI framework, caregiving activities and attitudes are part of the intervention process and plan. In this literature review, I sought to synthesize available research on the unique caregiving needs of infants with autism. I used the following question to guide analysis of this body of literature, "How does parenting an infant with autism contrast with parenting an infant with typical development?" This line of inquiry allowed me to situate family needs in the context of available special education services for young children with autism.

Theme 3: EI and autism, Individuals with Disabilities Act, Part C (IDEA, Part C). Very recently special education researchers concerned with EI for infants and 
toddlers with autism emphasized the need to resolve the incongruence between proposed EI models and federal legislation requirements under the Individuals with Disabilities Education (U.S. Department of Education, 1999). This review focused on the studies in the dominant autism research paradigm (i.e., special education and behavioral research) but will offer critiques from other peer-reviewed, scholarly sources. The dominant autism research groups expressed a growing concern over the lack of congruence between proposed first-generation AUT-EI treatment models and IDEA, Part C (Rogers \& Vismara, 2014; Zwaigenbaum et al., 2005). To date, only one AUT-EI model was congruent with IDEA, Part C (Wetherby et al., 2014). As a result, this review included an investigation of IDEA, Part C in the context of autism EI. Several subthemes emerged from this body of research including (a) a definition of IDEA, Part C, (b) family-centered practices, and (c) autism and IDEA, Part C.

Theme 4: Parent perspectives. Parent perspective was the fourth theme within the AUT-EI literature. Although there were few studies investigating parent perspectives with infants and toddlers with autism, two trends stood out in overall perspectives on EI practices and stress resulting from parenting a child with autism. Parent perspectives on EI practices were consistent with the larger body of EI research. As in other interventions for infants and toddlers with disabilities, parents' perspectives about services positively correlated with a number of service dimensions including the relationship with an interventionist, service coordination, and treatment efficacy (Corcoran et al., 2015). Studies repeatedly found stress associated with parenting a child with autism was greater than parenting a child with typical development (TD) or developmental delays (DD). 


\section{Research Questions}

This study was guided by the following research questions:

1. What are the participants' lived experiences as parents with a young child (under age five) with ASD as they relate to early intervention services?

1a. What are the participants' experiences of having a young child diagnosed with ASD within the past two years?

$1 \mathrm{~b}$. What are the structural essences of the phenomena of parenting a very young child with ASD in the context of EI services?

\section{Theoretical Frameworks}

While there were many theoretical frameworks that could have been used to structure the discussion on parent lived experiences with young children with autism, I used two theoretical frameworks. The first was phenomenology. Phenomenology was well suited to examining lived experiences of parents of young children with autism receiving special education services. I also utilized Bronfenbrenner's Ecological Systems Theory (1979) to provide a context for the ways in which early development is embedded and influenced by many ecologies.

\section{Phenomenology}

Phenomenology is both a philosophy and a research methodology. "Phenomenology is an encounter, a way of living, and craft" (Vagle, 2014, p. 11-12). It is a way of "looking at what we usually look through" (Sokolowski, 2000, p. 12). Phenomenology as a philosophical movement was in direct response to empirically bound ideas about the meaning of the human experience and the world. Instead of investigating objective agreed-upon constructs, phenomenology emphasizes isolating truth as it is experienced by participants and elucidating phenomena through emergent essential themes. These two constructs, lived experiences and essential themes are 
philosophical constructs but grounded in qualitative research methods (Vagle, 2014). The researcher investigates the phenomena and isolates meaning of lived experiences through research activities. The researcher must bracket (Husserl, 1970) their conceptions to avoid misconstructions placed on the experience in advance. These preconceptions and impositions may originate in religious, cultural, or socio-political traditions. They also may be drawn from personal bias. In phenomenology, there are no a priori hypotheses, freeing the philosopher or researcher from prejudice. Phenomenology is a school of thought that rejects all dogmatisms, research traditions, and externally imposed methods.

[Phenomenology] is particularly suitable for research where the focus is on the uniqueness of a person's experiences, how experiences are made meaningful, and how these meanings manifest themselves within the context of the person both as an individual and in their many roles. (Shaw, 2001, p. 48)

Therefore, the focus of this methodological paradigm is the subjective experience with an examination of participants' detailed lived experience as a way of making sense of the world. The intent of a phenomenological investigation is "to uncover the meanings [of lived experiences] in everyday practice in such a way that they are not destroyed, distorted, decontextualized, trivialized or sentimentalized" (Benner, 1985, p. 6).

As a research orientation, phenomenology adheres to a social constructivist philosophy of human development and experience with sociocultural and historical processes central to one's interpretation of his/her own life (Eatough \& Smith, 2008). The methodology is an iterative process that seeks to understand the participant's point of view. Modes of interpretation may be empathetic and descriptive to develop a rich picture of the lived experience of each participant. Interpretation may also require critical analysis of the ways in which the participant was unable or unwilling to share something 
hidden.

Phenomenology as a theoretical framework fit with the objectives of this study for several reasons. This literature review yielded no studies focusing on the lived experiences of parents of infants/toddlers with autism receiving EI. Contemporary special education and behavioral research with infants with autism and their parents were predominated by a quantitative, controlled research designs. Most AUT-EI studies I found in my literature review lacked qualitative investigations into the participant's' daily lived experiences and parent perspectives.

\section{Ecological Systems Theory}

In the 1950's, developmental researcher Urie Bronfenbrenner (1979) introduced a new conceptualization of child development. Unlike predecessor stage-theorists, Bronfenbrenner proposed that child development occurred in the context of many interrelated social, economic, and cultural ecologies that exerted influences on the family and child. These contextual influences (i.e., ecosystems) bi-directionally interact. Figure 2 illustrates these processes and provides examples of factors that influence child development. 


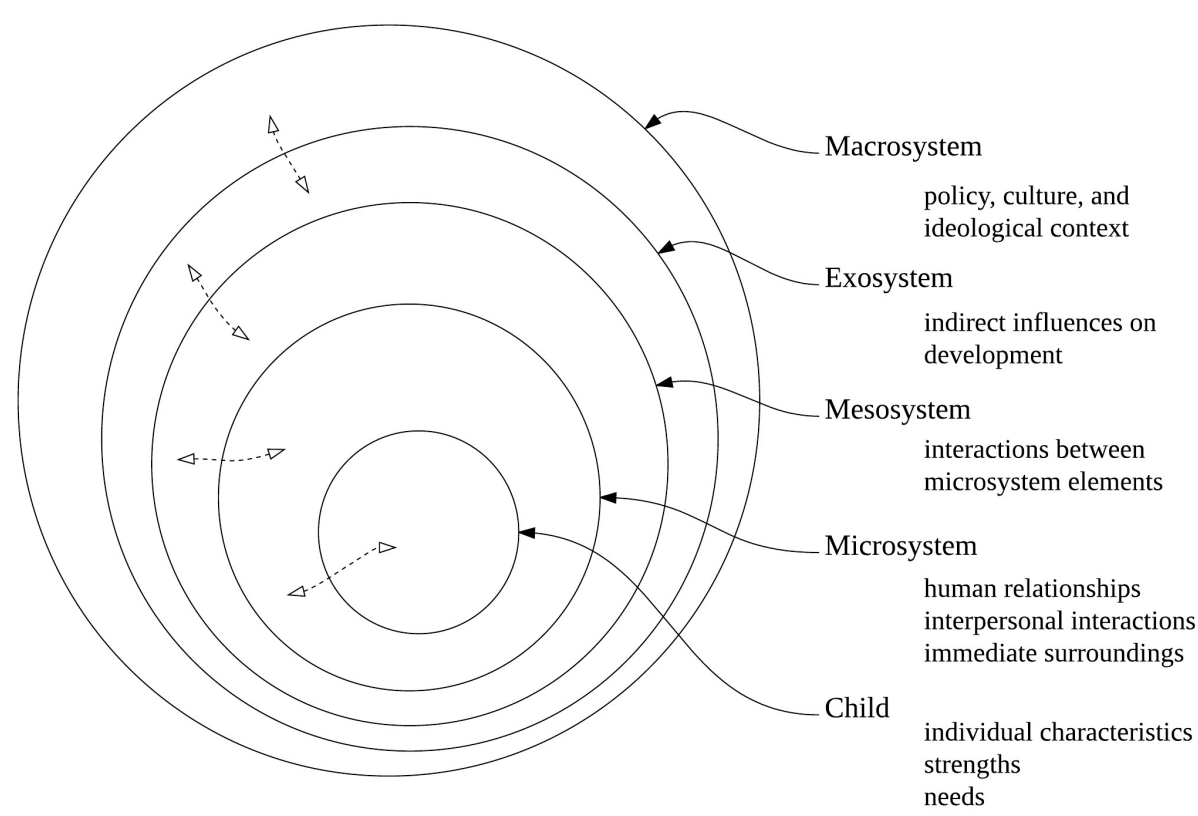

Figure 2. Bronfenbrenner's Ecological Systems Theory.

Prior to Bronfenbrenner, the normative view of child development was that it followed a series of predictable stages with little emphasis on the role of proximal and distal influences in determining child developmental outcomes. However, over the past forty years, a wealth of studies repeatedly supported Bronfenbrenner's theory (e.g., Hart \& Risely, 2004; Sameroff et al., 1993; Sameroff et al., 1987). Because of Bronfenbrenner's Ecological Systems theory and subsequent research, the power of environment on child development is now well established.

Bronfenbrenner (1979) proposed a theory that weighted environmental influences on child development and described these influences as nested ecological systems. Ecosystems exert bidirectional effects. The contexts described by Bronfenbrenner enhanced or diminished a parent's capacity to support their child's development. 
Proximal influences (i.e., microsystems) include supports and environments within which families are directly involved (e.g., church, daycare family, playgrounds). However, distal contexts (i.e., macrosystems) also contribute to healthy child development. Culture, attitudes, and mass media all shape each family's unique ecological profile. Further, the larger system operates through interconnections (e.g., child's temperament, socioeconomic status, and family culture) so that bio-ecological systems are continually and bi-directionally influencing each other.

Human development takes place through processes of progressively more complex reciprocal interaction between an active, evolving bio-psychological human organism and the persons, objects, and symbols in its immediate external environment. To be effective, the interaction must occur on a fairly regular basis over extended periods of time. Such enduring forms of interaction in the immediate environment are referred to as proximal processes. (Bronfenbrenner \& Morris, 1998, p. 996)

The Family Systems Intervention Model. Dunst and Trivette (2009) elaborated on Bronfenbrenner's ecological systems theory in their seminal research with familycentered practices in EI. The Family-Systems Intervention model situates child development in the contexts of intervention practices that increase parent self-efficacy and capacity to care for their child. Notably and consistent with IDEA, Part C, evidencebased practices that build parent capacity to support child development require an understanding of (a) family strengths, (b) family concerns and priorities, and (c) supports and resources. Dunst and Trivette's work led to evidence-based practices in early intervention and this gave rise to the foundation for best practices in EI. The Family Systems Intervention model demonstrated how building family capacity within the most proximal systems increased child development outcomes. In this model intervention 
involves identifying family strengths, concerns and priorities, and resources. Two principles guide interventions: capacity building. This elaboration on Bronfenbrenner's theory bridges theory and practice in EI with families and young children and is depicted in Figure 3.

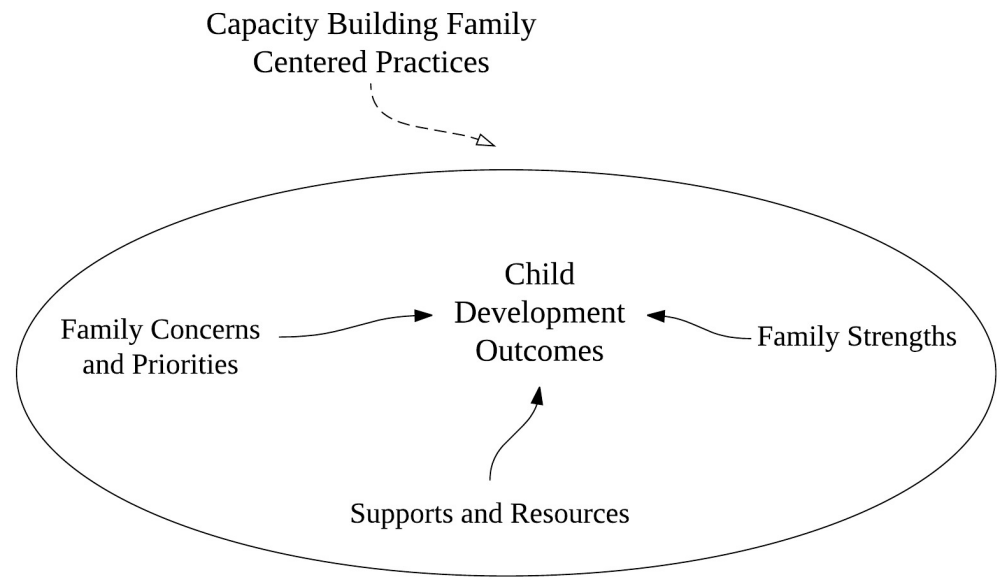

Figure 3. Family Systems Intervention Model (Dunst and Trivette, 2009). Building family capacity through family centered practices positively influences child developmental outcomes.

\section{Review of Research Literature on Early Interventions for Autism}

\section{What is AUT-EI?}

In response to the confirmation of autism markers in infancy and theories of cascading effects of prodromal characteristics in later development, predominant autism intervention researchers began developing the first EI models to address autism in infants and toddlers. The first-generation AUT-EI models adapted focused intervention practices and comprehensive treatment models with evidence in improving development and reducing problem behavior in older children.

This section discusses emergent autism and reviews the intervention research 
literature. This section also critiques the AUT-EI comprehensive models, focused interventions, and the ABA treatment paradigm for children with autism.

\section{How Early Autism Emerges}

Infant symptoms of autism (birth to three). Since the late 1980s, there has been considerable research on the onset of autism and markers in the first two years of life. Seminal retrospective studies including first birthday home-movie analysis, as well as parent reporting, were the first to identify differences in babies that later went on to receive a diagnosis of autism (see Saint-Georges et al., 2010). More recently, researchers studied the emergence of autism markers in a high-risk population (i.e., infant siblings of children already diagnosed with autism; INF-SIBS; Rogers, 2009). Early INF-SIBS studies focused on confirming markers and sequencing possible atypical trajectories in very young children with autism. The following section summarizes the literature on autism symptoms during infancy.

Retrospective studies. In the 1980s, a small group of researchers turned their attention to studying whether symptoms of autism were evident before school age (e.g., Adrien et al., 1993; Baranek, 1999; Osterling \& Dawson, 1994). Historically, autism had not been identified until full manifestation of the syndrome (i.e., language impairments, marked differences in social functioning, and restrictive interests and/ or repetitive behaviors). Until recently, infants and toddlers with autism were largely overlooked and a diagnosis was delayed until school age (Mandell et al., 2002; Stone \& Rosenbaum, 1988). The earliest studies of infant markers involved parent interview methodologies (e.g., Dahlgren \& Gillberg, 1989; Stone \& Lemanek, 1990). These research studies confirmed that early 
autism behaviors crossed several developmental domains including: social skills, communication, affective expression, and sensory responses (Dahlgren \& Gillberg, 1989). Because of parent report studies, a veritable explosion of research into early manifestations of autism ensued.

Several seminal studies using retrospective analysis of family home movies (Baranek, 1999; Dawson \& Munson, 2002; Osterling \& Dawson, 1994) led to a new wave of research on infant symptoms of autism. Research teams analyzed home movie footage of children before they were diagnosed with autism. At that time, autism was not typically diagnosed until school age. Because of the delayed diagnosis, there was no infant population to study. In response to this research dilemma, in a seminal study, Osterling and Dawson (1994) conducted one of the first infant autism home movie studies in which clips were selected to control for child age. They identified a number of infant behaviors that discriminated infants with autism from those who were typically developing.

Retrospective studies have repeatedly found infants under 24 months show differences in: smiling at others (Adrien et al., 1993; Maestro et al., 2002), response to their name being called (Baranek, 1999; Osterling \& Dawson, 1994; Osterling, Dawson \& Munson, 2002), looking at others/joint attention (Adrien et al., 1993; Maestro et al., 2002; Osterling \& Dawson, 1994; Osterling et al., 2002; Zwaigenbaum et al., 2005;), and imitative play (Zwaigenbaum et al., 2005). Several studies noted early sensory-motor deficits in infants later diagnosed with autism (Baranek, 1999; Dallgren \& Gillberg, 1989; Wimpory, Hobson, Williams, \& Nash, 2000).

Prospective Studies. Prospective studies of infants with an older sibling 
diagnosed with autism confirmed and increased understanding of social-communication impairments in infants/toddlers with autism. Early Interventions for Autism researchers selected INF-SIBS because of their increased genetic risk for developing autism. Close to $20 \%$ of infants with an older sibling with autism developed the condition compared to only $2 \%$ for infants with an older sibling with typical development (Ozonoff et al., 2011). Prospective studies of INF-SIBS offered autism researchers a way to conduct larger, experimentally rigorous investigations of the emergence and course of autism in infancy. Studies of INF-SIBS of children already diagnosed with autism confirmed and expanded previous retrospective research findings (see Rogers, 2009). Prospective research studies reported that infants with autism exhibited differences in orienting to name, imitation, affect, gesture use, social smiling, social interest, and sensory behaviors (e.g., Bryson et al., 2007; Ozonoff et al., 2011; Presmanes, Walden, Stone, \& Yoder, 2007; Zwaigenbaum et al., 2005).

The prospective literature findings suggested a possible trajectory of autism starting in infancy. Infant social-communication in autism began to diverge from typical at around 12 months of age. By age three, young children with autism engaged in less functional and symbolic play, exhibited language and social skill impairments, and displayed repetitive and restrictive interests (Barbaro \& Dissanayake, 2013). Landa, Gross, Stuart, and Faherty (2013) studied trajectory patterns in INF-SIBS with early symptom onset, late symptom onset, and no autism. The results indicated that the three groups were indistinguishable at six months but both autism groups exhibited atypical developmental trajectories thereafter. The early onset groups showed more severe 
impairment than the late onset group, but the two autism groups were comparable after 36 months.

\section{Theories of Impact of Gaps in Services for EI on Later Development}

The social motivation theory. Retrospective and prospective studies confirmed social-communication differences in emergent autism. This research led to hypotheses about the effects of infant autism symptoms on later development. Some major research groups in AUT-EI operated under the theoretical assumption that early deficits in socialcommunication associated with autism interfered with interest and orientation to socially relevant stimuli. Rogers and Dawson (2010) described their theory underlying AUT-EI:

Consider, then, how having autism might affect how the brain develops. Infants with autism respond differently to the environment, many from the first year of life. The infant is less responsive to social stimuli and does not initiate social interaction. Thus, he or she has fewer social interactions over the course of a day than would be typical...brain connections and neural response patterns are likely also diverging, and this may be another source of ongoing brain differences. ( $\mathrm{p}$. 12)

This theoretical assumption guiding some researchers and interventionists evolved into overlapping theories on the developmental effects of infant social-communication defects in autism. Elsabaggh and Johnson (2007) described a disruption in the bias towards social orienting in infants with autism. Similarly, the Social Motivation Hypothesis is theoretical basis for Dawson et al.'s (2005) intervention models. The Social Motivation Hypothesis proposes that infants with autism experience core impairment in social motivation that interfered with typical brain development. Social motivation was described as a set of "psychological dispositions and biological mechanisms biasing the individual to preferentially orient to the social world, to seek and take pleasure in social 
interactions, and to work to foster and maintain social bonds" (Chevallier et al., 2012, p. 231).

The theoretical assumptions underlying the social motivation hypothesis may have implications for how researchers and interventionists view and treat children with autism. In a rather unsettling review of the research literature on autism related social impairments, typical social development, and brain function, Chevallier and colleagues (2012) described social motivation in humans and primates and went on to explain social impairments in autism as a lack of interest in forming and maintaining human relationships. Chevallier et al. attributed the intact attachment and sexual drive in individuals with autism as evolutionarily advantageous. According to the authors the theory of social motivation and autism, instinctual survival mechanisms are spared in individuals with autism. The researchers linked a hypothesized lack of social motivation and interest in relationships with others with differences in brain structures associated with control emotional and social functioning. The researchers concluded that the social motivation theory is a credible framework to explain the social impairments in autism.

According to the social motivation theories, early deficits associated with autism have downstream effects on an infant with autism's developmental trajectory in a number of ways (Chevallier et al., 2012; Dawson, 2005). The infant's lack of social orienting disrupts early social interactions with parents that, in the immediate context, reduce salient social input. This stimuli is a necessary condition for the development of later, more sophisticated language and social skills. According to these theories, the result of deprivation of social-communication is an increasingly divergent atypical trajectory. The 
primary effects of autism had an immediate impact on interactions, but also led to cascading effects that altered later child developmental outcomes, particularly in language and social development.

\section{Critique of the social motivation theory underlying social-communication in}

autism. While social communication differences are confirmed in infants who go on to receive an autism diagnosis (Rogers, 2009), no evidence was found in the literature that confirmed the social motivation hypothesis. Many researchers acknowledged that mechanisms underlying early autism are largely unknown (Camarata, 2014; Elsabaggh \& Johnson 2010; Zwaigenbaum et al., 2015). So, while the social communication markers of autism manifest as marked differences in early social orienting, it is not known whether the babies find social interaction aversive or if some other mechanism is responsible. In an alternative theoretical model, Talay-Ognon and Wood (2000) proposed that negative sensory experiences have a cascading effect on social development.

Consistent with the phenomenological philosophy, literature from disciplines in addition to behavioral and dominant intervention research paradigms offered insight into another possible explanation for social communication deficits in infant autism. Sensory processing problems in autism might play a role in withdrawal from early social interactions.

Recent research found sensory processing dysfunction is correlated with autism in a very young children with autism (Wigham, Rodgers, South, McConachie, \& Freeston, 2015). Sensory processing is an impairment in modulating environmental stimuli (Dunn, 1997; Wigham et al., 2015) and sensory input can be experienced as "heightened" and 
"unpleasant" or alternatively as "reduced" (p. 934). Poor sensory registration are manifest in problems registering sensory stimuli do to a high neurological threshold. In low neurological thresholds (high sensory registration) children are highly sensitive to sensory stimuli.

Sensory processing issues are associated with discomfort and have been widely reported in children with autism (Ben-Sasson, Cermak, Orsmond, \& Tager-Flusberg, 2007; Kern et al., 2006; O’Donnell et al., 2012). Sensory processing problems in autism include low registration and high registration (Dunn, 1997). There was evidence that early social functioning is associated with low sensory registration. Dunn (1997) proposed a conceptual model of sensory processing linking poor registration with social behaviors. Behaviors associated with poor registration included (a) doesn't express emotions, (b) doesn't perceive body language or facial expressions, (c) doesn't have a sense of humor (d) doesn't seem to notice when someone touches arm, and (e) doesn't notice when people come into the room.

In a study of sensory modulation with toddlers with autism, Ben-Sasson (2007) found sensory differences between children with autism and children with TD. In children with autism, low-registration was the most prevalent and extreme sensory behavior. Children selected for the study included one hundred toddlers with autism and 101 controls with matched mental age and 99 with matched chronological age. Children and parents were involved in the research and were assessed on autism symptoms, sensory measures, and infant / toddler social emotional assessment measures. The researchers found sensory processing differences between toddlers with autism and 
matched controls. Low registration was associated with the high rate avoidant behaviors and the social aspects of these behaviors. Children with also presented with mixed high and low registration suggesting problems with overall sensory modulation in autism.

Green, Ben-Sasson, Soto, and Carter (2012) found greater activation in the sensory areas of the brain in toddlers with autism. There was also an increase in activation of the brain's emotional regulating system indicating increased anxiety in toddlers with autism. Earlier studies found associations with facial processing and activation of the amygdala, a brain structure associated with fear (Adolphs, Sears \& Piven, 2001; Dalton et al, 2005). Schultz (2005) found associations between eye gaze and activation of the amygdala in individuals with autism. While a comprehensive review of the sensory processing literature is beyond the scope of this proposal, sensory processing problems associated with autism offer an alternative theory of the etiology of social communication deficits in early autism.

\section{Clinical implications of theories underlying social communication and infant}

autism. While no studies were found to confirm or challenge the social motivation hypothesis, attributing social impairments in autism as a deficit in a bias towards social orienting may have implications for the approach parents and interventionists use when delivering autism interventions with young children. If there is a different etiology of social communication deficits in infant autism then some of the assumptions about how best to treat emergent autism may be incorrect or in need of adjustment. If we believe children with autism inherently don't want or don't like social relationships, the focus of intervention may be to find ways amplify saliency of dyadic interactions and focus on its 
reinforcement value. Whereas if we attribute these social deficits to sensory discomfort and not to preference for objects then our approach would be to make young children more comfortable so they can access social interactions that would otherwise be reinforcing. There is evidence that parents have been recognizing the connection between sensory processing issues and intuitively adapting parenting behaviors to make their child

more comfortable. (Dickie, Baranek, Schultz, Watson, \& McComish, 2009). Green et al. (2012) recommended that sensory processing be addressed in childhood interventions. This recommendation was to reduce the effects of early sensory processing dysfunction and prevent anxiety problems later on.

\section{Early Intervention and Infants with Autism}

Overview. This section will discuss and critique available treatments for infants and toddlers with autism. This is an emergent area of intervention study. This section will review and critique (a) comprehensive treatment models, (b) focused intervention practices, and (c) the ABA paradigm.

Comprehensive treatment models. Comprehensive AUT-EI treatment models are systematic, structured, interventions that target developmental goals. These models differed from focused intervention practices that teach a discrete skill or address shortterm goals. In contrast, comprehensive treatment models (CTM) for AUT-EI were models spanning over longer periods of time with manualized curricula and addressed core impairments of autism and developmental goals. All CTMs retained ABA pedagogic practices. Dawson (2010) outlined a set of best practices in efficacious CTM for infants and toddlers with autism (a) parent coaching on use of intervention strategies in daily, 
home contexts; (b) frequency and length of intervention; (c) individualized activities designed to meet the developmental needs of each child; (d) beginning the interventions as early as possible; and (e) increasing parent responsivity and sensitivity to infant cues. While there were clear benefits to AUT-EI treatments, most first-generation AUT-EI models had little impact on child developmental outcomes (Camarata, 2014; Warren et al., 2011).

Most of the CTM in the literature review for this dissertation proposal incorporated principles of ABA combined with developmental frameworks for intervention (Schreibman et al., 2015; Zwaigenbaum et al., 2015). The CTM intervention literature was predominated by quantitative experimental research. This trend was consistent with traditions in autism research and intervention practices with older children (NRC, 2001; Wong et al., 2015). Comprehensive treatment model studies adhered to the same scientific rigor in an effort to identify treatment variables that moderated efficacy. Eclectic and non-ABA approaches to intervention were discouraged by expert groups and did not have an evidence base in the literature (Howard, Sparkman, Cohen, Green, \& Stanislaw, 2005; Wong et al., 2015; Zwaigenbaum et al., 2015). There were few qualitative studies found in the CTM literature and none examined the lived experiences of parents directly or ancillary to CTM curricula under investigation. The vast majority of CTM research focused on identifying evidence-based interventions for infants and toddlers with autism.

\section{Comprehensive treatment models for young children with autism selected for}

the literature review for this proposal. This review identified six recent CTM 
intervention studies that met criteria consistent with early intervention best practices for all infants/toddlers with special needs (BP-EI). To be included in the AUT-EI review for this proposed study, the studies had (a) a mean age of infant $<30$ months, (b) parent mediated methods, and (c) interventions situated in natural family routines. All other AUT-EI models were excluded. The following studies met the inclusion criteria for this review of the research literature:

- Video Interaction to Promote Positive Parenting (Green et al., 2015)

- Focused Playtime Intervention (Kasari et al., 2006)

- Early Start Denver Model-Parent Intervention (Rogers et al., 2012)

- Joint Attention Mediated Learning for Toddlers with autism (Schertz et al., 2013)

- Pivotal Response Treatment for Infants At Risk for Autism (Steiner, Gengoux, Klin, \& Chawarska, 2013)

- Parent Implemented Social Intervention for Toddlers with Autism (Wetherby et al., 2014)

Description of treatments selected for this proposal review. Only one model reviewed was appropriate for infants under 12 months (Green et al., 2015). This intervention targeted parent/infant interactions with strategies to enhance parent responding and infant bids for social interaction. The remaining five intervention studies reported on treatments for toddlers with or at risk of autism (i.e., between 12 and 31 months). Consistent with the autism infant symptoms research, all interventions targeted social-communication in infants/toddlers.

All of the studies except Green et al. (2015) used manualized parent training curricula. Parents were coached in ABA strategies to support their child's socialcommunication development in parent / child routines. Schreibman et al. (2015) described manualized practices as: 
Having clear procedures carefully described in their respective intervention manuals. Accurate implementation of an intervention requires clearly stated procedures. Manualization helps with training and consistency of treatment implementation among treatment providers (professionals, parents, etc.). (p. 2418)

Parent training and support ranged from video feedback, guided practice, reflection on intervention, worksheets, and assignments. Intervention coaches recorded parent fidelity of implementation for all six studies reviewed in this section. All treatment models were designed for implementation in the home context. All but one of the intervention studies situated parent training in the home (Rogers et al., 2012).

None of the six models reported a qualitative component to solicit information on parent priorities, concerns or resources, a requirement of IDEA, Part C (US-DOE, 1999). The Parent Implemented Social Intervention for Toddlers with Autism (Wetherby et al., 2014) included a measure of family-centered practice. However, this program's curriculum was more consistent with child-centered than family-centered practices.

Given the early onset of autism and effects on social communication, predominant researchers theorized that intervening in infancy had the potential to ameliorate infant autism's effects on later development. However, despite support for CTM some researchers voiced criticism about individual study data and effects on child development (Camarata, 2014; Warren et al., 2011). Many research groups noted that autism's heterogeneity and problems with identification in infancy posed a threat to generalizability of AUT-EI research findings (Bradshaw et al., 2014; Camarata, 2015). Comprehensive treatment model research teams also reported mixed effects on child development outcomes (Camarata, 2014; Warren et al., 2011). Only one parentimplemented a home-based CTM to date (Wetherby et al., 2014) reported significant 
improvement of the child on standardized developmental scores in language. However, nearly all AUT-EI studies had positive effects on parent fidelity, parent/infant responding, and some child social communication or play behaviors (Rogers \& Vismara, 2014). The remainder of this section presents a critique of the AUT-EI research.

Generalizability of results of comprehensive treatment research. Camarata (2014) was one of the first researchers to question the generalizability of first-generation CTM studies' results. Camarata also reported on a number of confounding issues with methodologies used across studies and cited problems with comparisons across studies given the variability in ages, length of treatments, and intervention approaches. Other researchers cited similar methodological barriers to evaluation of current CTM study outcomes (Warren et al., 2011; Zwaigenbaum et al., 2015). The small number of first generation treatments rendered meta-analysis impossible. Other researchers noted problems with the identification process and studies of earliest behavioral criteria (Dawson, 2008; Elsabbagh \& Johnson, 2007).

Autism is a heterogeneous condition throughout the lifespan. Heterogeneity was also evident in infant symptoms. In addition to varying symptom expression and stability, the course of autism was inconsistent and unpredictable, particularly in younger infants. Camarata (2014) reported that stability of diagnosis was a problem unique to CTM. Autism stability of diagnosis varied among subgroups of the autism spectrum until after age five (Camarata, 2014). Rondeau et al. (2011) conducted a meta-analysis of stability rates in autism and pervasive developmental disorder, not otherwise specified (PDDNOS). Pervasive Developmental Disorder, Not Otherwise Specified is a variable and 
often less pronounced autism condition. Rondeau et al. (2011) reported diagnostic stability under age three varied by condition. In children with autistic disorder (i.e., classic autism presentation), there was $76 \%$ stability of diagnosis. In children diagnosed with PDD-NOS under age three, only $35 \%$ retained an autism diagnosis.

Many research studies reported a wide range of behavioral profiles and sometimes-unexpected developmental courses of autism. In a review of autism infant research, Rogers (2009) reported self-correction in some infants with markers specific to autism (i.e., not observed in infants who were typically developing or with developmental delay). These infants "outgrew" autism by school age. There was no explanation for this unexpected developmental course. Studies also confirmed regression in a small subgroup of infants with autism. These infants were reported to have developed typically until shortly after 12 months when there was a rapid loss of language and social skills (for review see Rogers, 2004). For these reasons, Camarata (2014) argued that CTM efficacy data needed further examination. Both Warren et al. (2011) and Camarata (2014) argued for more research examining subgroups of infants/toddlers with autism and identifying which interventions and strategies best fit across groups with different characteristics. In 2015, an international panel of autism experts reported consensus on a number of CTM related issues (Zwaigenbaum et al., 2015). The published executive summary stated heterogeneity of autism in infancy has ramifications for the interpretation of AUT-EI research as well as for clinical practice.

\section{Reported effects of comprehensive AUT-EI treatments on child development}

outcomes. Warren and colleagues (2011) questioned the accuracy of reported effects of 
AUT-EI on child development outcomes, arguing that while the research community agreed on a need for AUT-EI, there was little evidence for effectiveness of current interventions. Warren et al. (2011) reviewed parent-mediated, home-based AUT-EI literature. The review yielded evidence for methodological problems and poor treatment outcomes. Some studies failed to include baseline conditions (e.g., Aldred, Green, \& Adams, 2004; Wetherby \& Woods, 2006). Many AUT-EI studies positively affected parent/infant interaction outcomes (Carter et al., 2011; Green et al., 2015; Kasari, Gulsrud, Wong, Kwon, \& Locke, 2010; Wetherby et al., 2014). Most reported little or no improvement in child social, language, or cognitive skills (Aldred et al., 2004; Drew et al., 2002; Osterling et al., 2010; Stahmer \& Gist, 2001). Ironically, Drew et al. (2002) reported children in the treatment group lost IQ points whereas the children in the control group did not. There was no data on long-term effects of AUT-EI beyond five years.

\section{Comprehensive review of first-generation CTM and recommendations for}

practice. Zwaigenbaum et al., (2015) conducted a review of AUT-EI treatments for young children under age 3 . These models were a combination of behavioral and developmental approaches, involved families, and focused primarily on social communication. Twenty-four studies were included in the review and included randomized control, quasi experimental, and open label designs. Six comprehensive treatment models and four teaching methods exhibited significantly improved outcomes compared to control groups. The remaining exhibited less than moderate quality of evidence. Based on the findings of the review, Zwaigenbaum and colleagues (2015) made recommendations for clinical practice with infants and toddlers with autism. The group 
recommended that behavioral-developmental practices and including parents in intervention. Consistent with limitations noted by other AUT-EI research groups, 7 of the 10 recommendations addressed the need for a better understanding of moderators of treatment, clinical presentation of autism, biological and behavioral heterogeneity, and socio-cultural factors. Zwaigenbaum and colleagues (2015) recommended future research identifying active components of interventions (e.g., agent implementing intervention, consistency of service provision across environments, duration of treatment and hours per week). Zwaigenbaum et al. stressed that future AUT-EI research should examine biological and behavioral heterogeneity in order to better understand intervention outcomes. This recommendation was consistent with both Camarata's (2011) and Warren et al. (2011) findings from their reviews of first-generation AUT-EI intervention studies.

\section{Focused evidence-based intervention practices in autism interventions.}

Evidence based practices (EBP) in the field of special education represent interventions and models that have a scientific evidence based for improving student outcomes. Odom and colleagues (2010) define EBP as general and special education practices with scientific evidence of efficacy. The goal of identifying practices with scientific evidence of effectiveness is to inform practice and insure the most effective interventions are used by teachers and clinicians. By employing EBP in applied settings the aim is to safeguard against the use ineffective or potentially harmful treatments (Vismara \& Rogers, 2010). Evidentiary criteria of research methodology are used to determine which intervention studies are deemed to have evidence of improving outcomes. A number of research and policy organizations developed criteria to determine which research methodologies meet 
quality standards. Evidence based practices in the field of interventions for children with autism are the basis upon which interventionists and teachers design their educational programs (Odom, Collet-Klingenberg, Rogers, \& Hatton, 2010).

Focused interventions are a separate category of practice from CTM (Wong et al., 2015). Focused intervention practices are operationalized and typically focus on a single skill or goal (e.g., discrete trial teaching, pivotal response, prompting). Focus interventions are the foundation for and most salient features of comprehensive treatment models and eclectic intervention programs. Examples include discrete trial teaching, pivotal response training, prompting, and video modeling (Wong et al., 2015).

The most recent and comprehensive study on EBP in autism intervention reported on 27 focused intervention practices with scientific evidence of improving developmental outcomes in children with autism (Wong et al., 2014). The review activities reflected the philosophy and standards of the earlier movement towards scientifically-proven practices in medicine (Wong et al., 2015). Consistent with the paradigm, the Council for Exceptional Children established criteria for evaluating evidence of efficacious autism intervention studies. The National Professional Development Center for Autism Disorders (NPDC) employed an adapted research design quality indicator (Chambless et al., 1996) to determine articles to include in the review. The NPDC group identified 24 focused intervention practices that met the criteria for being evidence-based. Interventions incorporating the principles of $\mathrm{ABA}$ represented $58 \%$ of the evidence-based practices. The remaining $42 \%$ may have included ABA practices but the defining features of these were structured procedures (e.g., Parent Implemented Intervention, Peer- 
mediated instruction).

To be included in the intervention research review focused interventions had to be behavioral, developmental, and / or educational in nature. Studies needed to meet the following criteria to be considered evidence-based (a) supported by two high quality experimental or quasi-experimental design studies conducted by two different research groups or (b) five high quality single case research designs by three research teams with 20 participants across studies or (c) a combination of designs according to the review's criteria.

Wong et al. (2015) reported on EBP by age and intervention outcomes. The majority of studies meeting criteria for EBP were single case designs with children ages 6-11. Studies with infants and toddlers involved considerably less participants, reflecting the emergent stage of AUT-EI intervention research.

Limitations of Wong et al.'s (2015) outcomes on EBP in autism intervention. Wong and colleagues (2015) reported limitations in their comprehensive review of EBP in autism intervention. These limitations reflected validity issues reported by other research studies and reviews (Camarata, 2014; Zwaigenbaum 2015).

- The compressive review did not include demographic data in the analysis of intervention efficacy. Race, ethnicity and socioeconomic status were not factored into review as possible moderators of treatment outcomes.

- A minority of the studies meeting the experimental design criteria included data on comorbidity. Most studies did not include co-morbidity data and of those that did $25 \%$ reported intellectual disability.

- The inclusion criteria for studies was an autism diagnosis (as defined at the time of the review: autism, Asperger's syndrome, PDD-NOS) but not subgroups of participants were analyzed for response to treatment. Autism heterogeneity and potential selection bias has been a concern across autism intervention research and that issue was not addressed in Wong et al.'s EBP report. 
- A reported limitation was the need to translate results into intervention practices.

- The relative strengths of EBPs were not reported. As a result a practitioner would not have information to determine to what degree implementing the practice with fidelity will produce determined outcomes.

- Intervention outcome data focused primarily on core symptoms of autism (i.e., social-communication, challenging behaviors). The studies lacked information on strengths and abilities.

- Most of the intervention studies included in the review were with boys. Little is known about the effects of focused interventions on outcomes for girls.

Critique of ABA as dominant treatment paradigm for autism. This section will discuss the role of ABA in proposed AUT-EI treatments and offer a critique of the reported evidence-based focused intervention practices (Wong et al., 2015). However, while $\mathrm{ABA}$ dominates intervention research and practices, other interventions have shown positive effects on child development. Lindgren and Doobay (2011) conducted a review of interventions for autism and while they concluded $\mathrm{ABA}$ interventions are the "gold standard" for autism treatment, several other intervention practices also reported evidence for effectiveness (e.g., augmentative and alternative communication, picture exchange, visual supports). These strategies are often used in conjunction with ABA based interventions (Wong et al., 2015). In a review of focused intervention practices for autism, Wong and colleagues (2015) also indicated that there are currently a number of practices showing promise but not meeting the criteria for evidence based practice in their comprehensive review of focused autism interventions.

ABA as the dominant paradigm in autism research and intervention. Within the peer-reviewed journals in the larger field of autism intervention research, ABA has been described as the "gold standard" (Lindgren \& Doobay, 2011) in autism intervention both for comprehensive treatment models as well as focused intervention strategies. The 
prominence of $\mathrm{ABA}$ in intervention research and practices is widely accepted and explicitly stated in the intervention rhetoric. The evidence for ABA treatments for autism and a critique will be discussed later in this section. Table 1 presents examples of rhetoric found in articles that define ABA as the treatment of choice. These descriptions were gathered from peer-reviewed articles and book chapters by the leading research teams in the field (i.e., researcher teams who have developed, implemented, and tested interventions in wide use today).

Table 1

Rhetoric Describing ABA Practices in the Field of Autism Treatment.

$\begin{array}{cc}\text { Descriptions of ABA as Treatment of Choice in Autism } & \text { Research } \\ \text { Team }\end{array}$

Although there are a large and growing number of scientifically questionable treatments available for children with autism spectrum disorder (autism), intervention programs applying the scientific teaching principles of applied behavior analysis (ABA) have been identified as the treatment of choice.

It is clear that intensive, highly structured programs based on the principles of applied behavior analysis are the gold standard for autism treatment.

While there is a substantial research base supporting the effectiveness of behavioral interventions across the lifespan of autism, empirical data on the efficacy of interventions that meet the needs of toddlers with autism have begun to emerge only recently.

Most of these studies of toddler intervention are based on behavioral interventions that utilize more "naturalistic" approaches and developmental orientations than traditional applied behavior analytic (ABA)-based behavioral interventions.

[Regarding a comprehensive review of pilot AUT-EI intervention models for young children ages 4-30 months] most of the reviewed studies discussed behavioral principles as a foundational element of intervention (Carter et al. 2011; Dawson et al. 2010; Drew et al.
Rogers \&

Vismara, 2010

Lindgren \& Doobay, 2011, p. 20

Schreibman et al., 2015 (p. 2412)

Bradshaw et al., p. 786) 
2002; Koegel et al. 2013; Rogers et al. 2012; Steiner et al. 2013;

Wetherby and Woods 2006). Behavioral components that provided a framework for intervention included: providing a learning opportunity (antecedent), waiting for the child to respond (behavior), and delivering appropriate reinforcement (consequence). Further, all interventions used naturalistic approaches such that the infant's daily routines, natural interests, or preferred activities were infused into treatment procedures.

It is now widely acknowledged that the forms of treatment with the (NRC, 2001) most empirical validation for effectiveness with individuals with autism are those treatments based on a behavioral model.

Definition of $\boldsymbol{A B A}$. Applied Behavior Analysis refers to methods that change behavior in systematic and measurable ways (Steege, Mace, Perry, \& Longenecker, 2007). ABA models emphasize several principles of learning (e.g., reinforcement, modifying the learning environment, antecedent modification) These principles, along with many others, are the foundation for strategies and interventions under the "ABA umbrella" of intervention strategies (e.g., task analysis with prompting, incidental teaching, discrete trial, pivotal response training). Applied behavior analysis includes focused interventions, combinations of individualized interventions, and CMT built on ABA techniques. Applied behavior analysis has a large body of published empirical evidence for positive effects on developmental outcomes.

ABA as a sociocultural phenomenon. In addition to the prominence of $\mathrm{ABA}$ in autism treatment options, there is another aspect of $\mathrm{ABA}$ and autism that related to the lived experiences of parents with an infant or toddler with autism. Researchers and clinicians view ABA as the gold standard and treatment of choice for autism (NRC, 2001; Rogers \& Vismara, 2010). Applied behavior analysis may be viewed as both a set 
of treatment principles and a treatment monopoly.

This section provides a critique of ABA as a tradition and ideological institution. Philosophical discourses in the last century have linked educational systems and theories with power dichotomies. Some scholars argue that definitions of pathology and recovery are socially constructed and ultimately serve an exclusionary function for people with disabilities. Most notably, Foucault's philosophy on power and education offers a lens through which a critique of widely accepted models like ABA are possible:

A critique is not a matter of saying that things are not right as they are. It is a matter of pointing out on what kinds of assumptions, what kinds of familiar, unchallenged, unconsidered modes of thought the practices we accept rest (Foucault, 1988, p. 154)

The following sections offer a cursory critique of the ABA paradigm as a educational / socio-cultural phenomena.

Applied behavior analysis outside of North America. While ABA is the largely uncontested paradigm for treating autism in the U.S. and Canada, not all countries adopt it as unilaterally (Keenan, Dillenburger, Doherty, Byrne, \& Gallagher, 2010). As evidenced in the rhetoric of the predominant researchers in the field, $\mathrm{ABA}$ is considered the gold standard of ABA treatment. Government, advocacy, and multinational corporations promote ABA as the practice of choice for autism. The Surgeon General recommends $\mathrm{ABA}$ for treatment of children with autism (ABCA, 2016). Forty-four states fund ABA through government or health care systems as 'treatment as usual' (Autism Speaks, 2016). Multinational companies such as JPMorgan, Apple, Goldman Sachs fund ABA treatment for autism (Autism Speaks, 2014).

Rhetorical analysis of ABA discourse. Descriptive phenomenology requires an 
ongoing reflective engagement with the phenomena under investigation. Bracketing activities allow for identification of preconceptions about the phenomenon (Vagle, 2014). Bracketing requires identifying, isolating, and suspending preconceptions or assumptions about the phenomenon (Gearing, 2004; Vagle, 2014). This stance is evident throughout the research process. Internal and external suppositions must be suspended much like operations in mathematics are suspended outside of an equation and then returned to once the parenthetical mathematical problem has been solved (Husserl, 1970).

Applied behavior analysis philosophy and intervention is the dominant paradigm in autism treatment. However, the paradigm extends beyond intervention approaches. Applied behavior analysis has a history of defining what behaviors are acceptable and those in need of correction. In an effort to bracket this paradigm and its assumptions from my research, I re-examined my experiences designing and implementing ABA programs. I consulted with colleagues and participants. Finally, I spent a great deal of time examining the ABA literature from varying perspectives. The disability right's literature offered an alternate perspective on ABA.

In a disability rights article Broderick (2011) examined the history of autism and the influence of $\mathrm{ABA}$ research and intervention in creating a deficit model of autism. Broderick conducted an analysis of the rhetoric in applied behavior analysis discourse. Broderick used rhetoric to understand the relationship between language and power in normative views about autism. This investigation was consistent with rhetorical tradition; in this view, language creates meaning. In the case of individuals with disabilities, a way to approach new interventions and services is to analyze the way "the disabled have been 
and are inscribed in language and culture" (Linton, 1988, p.4). In the context of my investigation using descriptive phenomenological methods, Brocerick's rhetorical analysis allowed me to examine and bracket underlying assumptions about autism linked to the ABA legacy.

Broderick (2011) analyzed three "watershed moments" in autism discourse. The first was the publication of Lovaas's (1987) treatment effect study, the second was Maruice's (1993) publication of her own biographical experience implementing the Lovaas method, and the third was the 2005 founding of Autism Speaks. These watershed moments created a rhetoric that has defined the view and treatment of autism. I will focus on the first watershed moment, Lovaas's publication of his 1987 treatment for autism. This seminal study began the concept of "recovery" from autism to normalcy. Broderick (2011) argues that the concept of recovering from autism would not remain so resonant and enduring if it didn't tap into parents' hope that their child too can be normal. Broderick also points out that the use of the phrase recovery does not reflect the actual outcomes as they appear in the data. More accurately, half of the children in Lovaas's original study successfully completed third grade. Broderick describes Lovaas's repeated use of recovery and normalcy as profoundly impacting contemporary autism discourse. Broderick illuminates a contradiction or falsehood between Lovaass use of the term recovery and its operational definition. According to Lovaas' (1987) study, the children described as recovered tested at a normal IQ and successfully completed first grade. Broderick argues that if instead of using the term recovery (i.e., to normalcy) Lovaas reported that half of the children completed first grade, a different and less provocative 
view of autism treatment would have been realized. Instead Broderick argues:

Lovaas's rhetorical construct of "recovery [to normalcy]" has proven to be so powerful and so culturally resonant for nearly 25 years now precisely because it taps into parents' hopes for their children while simultaneously tapping into parents' fears, grounded though they may be in ableist cultural values (n.p.).

\section{Section Summary}

The review of the AUT-EI literature confirmed that markers of autism emerge around 12 months of life, sometimes sooner. Autism in infancy presents as prodromal behaviors in the areas of social-communication (e.g., response to name being called, social smiling, joint attention, and gesture use). Predominant AUT-EI researchers agreed that early autism interrupted development of later language, cognition, and social skills (Elsabbagh \& Johnson, 2007; Rogers \& Dawson, 2010; Zwaigenbaum et al., 2015). The research community emphasized that identification of effective AUT-EI treatment models was a high priority (Zwaigenbaum et al., 2015). A number of research teams reported on first-generation CTM treatment models. Early Interventions for Autism treatments focused on increasing social communication in infants with or at risk for autism. Interventions were delivered by parents trained in specific methodology and delivered in the home context. Some researchers in the field criticized AUT-EI study reports for a lack of evidence-base and methodological issues (Camarata, 2014; Warren et al., 2011; Zwaigenbaum et al., 2015). At the time of this review, AUT-EI was an emergent area of intervention research and these first-generation treatments had a limited evidence-base. 


\section{Review of Research Literature on Caring for an Infant with Autism}

\section{Introduction}

There was a large body of child development literature examining the experiences of providing care for very young children. Much of this literature was qualitative and informed by Bronfenbrenner's (1979) ECST . While caregiving studies were abundant in child development research literature (Hart \& Risely, 1995; Phillips \& Shonkoff, 2000; Piaget, 1960) and overall EI literature (Bailey, Hebbeler, Scarborough, Spiker, \& Malik, 2004; McWilliam et al., 1995), there was limited research into the experiences of caregiving an infant with autism. A number of studies reported on caregiving experiences with older children with autism (e.g., Grindle, Kovshoff, Hastings, \& Remington, 2009; Patterson \& Smith, 2011; Rodger, Keen, Braithwaite, \& Cook, 2008; Webster, Feiler, Webster, \& Lovell, 2004), but this number was also relatively small compared to the predominant quantitative traditions autism research.

Cocoran, Berry, and Hill (2015) conducted a meta-synthesis of fourteen qualitative investigations of lived experiences of parents of children (i.e., over age three) with autism from 2001 to 2012 . None of the studies reviewed directly involved parents of infants and toddlers with autism, some offered retrospective accounts of early symptoms, diagnosis, and navigating service.

Caring for an infant with autism may be substantially different from those with an older child with autism. Parenting an older child may involve less family adjustment and greater fluency in navigating autism service systems. Because of the gap in infant/toddler research, two areas of related research were used to garner insight into the themes related 
to caring for an infant/toddler with autism. This section is divided into (a) research indirectly related to caring for an infant/toddler with autism and (b) findings from qualitative caregiving studies with older children with autism. This section concludes with a summary and discussion of trends.

\section{Parent Studies Focusing on Caring for an Infant/Toddler with Autism}

Age parents had concerns about their infant's development. Until the last

decade, infant markers of autism were largely unrecognized by the research and medical establishments. Early autism infant research examined the age at which parents noticed problems and expressed concerns about their baby's development (Baghdadli, Picto, Pascal, Pry, \& Aussilloux, 2003; Dahlgren \& Gillberg, 1989). At the time of this literature review, parental concerns about their infant's development preceded autism diagnosis by several years. While this review found no research on the caregiving experiences of parents during that lag time, a number of seminal autism infant studies examined the age of parent recognition of symptoms (AOR) in their infants who later went on to receive an autism diagnosis. Parents noticed symptoms of autism between 18 and 24 months during emerging verbal and dyadic interactions (Baghdadli et al., 2003; DiGiacomo \& Fombone, 1998; Goin \& Myers, 2004). Most parents did not express concerns before six months and when parents did, it was not predictive of a later autism diagnosis (Ozonoff et al., 2009). Almost all parents reported recognizing a problem sometime between 18 and 24 months (e.g., Baghdadli et al., 2003; Charwaska et al., 2007, DeGiacomo \& Fombonne, 1998; Ozonoff et al., 2009; Rogers \& DiLalla, 1990;). Some studies reported parental AOR in the first year (DeGiaccamo \& Fombonne, 1998). 
Concerns arose earlier in children with lower cognitive functioning (DeGiaccamo \& Fombonne, 1998). Nearly all AOR studies reported parent's first concerns in social skills or speech and language development (Charwaska et al., 2007; DeGiacomo \& Fombonne, 1998). While these studies suggested possible impacts of parental concern for a child's development and lack of professional support, AOR research confirmed a lag period from initial concern to commencement of EI services when parents received little or no support for their child or family.

Quality of interactions between parent/infant with autism. It has been long established that the quality of parent/infant interaction during the first three years plays a powerful role in later developmental outcomes in infants with TD (Brooks-Gunn, 1995; Landry, Smith, \& Swank, 2006; Tamis-LeMonda, Bornstein, \& Baumwell, 2001). Recent research reported similar findings with infants with autism (Perryman, et al., 2013; Wan et al., 2013). Quality of dyadic interaction is bi-directionally influenced. The child and adult both make contributions that promote or impede positive functioning (Bornstein \& Tamis-Lemoda, 1997; Brazelton, Tronick, Adamson, Als, \& Wise, 1975). Given that autism is a condition that affects early social communication, a number of researchers explored the impact of infant autism on the parent/infant dyadic functioning. Parents and infants later diagnosed with autism exhibited disruptions in interaction synchrony and quality (Rozga et al., 2011; Yirmiya et al., 2006). Some studies reported differences as early as six months (Saint-Georges et al., 2011). Wan et al. (2012) found lower infant liveliness, higher parental directiveness, and differences in infant positive affect/attentiveness in dyads with infants with autism. Apicella et al. (2013) compared the 
behavior of dyads comprised of parents and infants with autism with dyads comprised of parents and infants with TD. After reviewing family home movies and analyzing the dyadic interactions, the researchers found that early in the first year of life, infants with autism showed less motor activity and fewer vocalizations. By the end of the first year, parents of infants with autism engaged in shorter periods of involvement and demonstrated a reduction in affectionate touch.

As early as the first year of life, parents with infants with autism engaged in behaviors that could be an intuitive response to early social communication deficits. Parents of infants who went on to receive an autism diagnosis became more directive (Wan et al., 2012) and provided more verbal scaffolding (Sigman \& Siller, 2002). These compensatory parenting responses correlated with onset of autism indicators in very young children.

Despite disruptions in parent/infant interactions, the quality of the relationship was similar to that of parents of young children who were typically developing and with developmental delays (Siller \& Sigman, 2002). Buerkens, Hobson, and Hobson (2013) attempted to tease out the relationship between autism severity and parent-child relations using a self-report questionnaire to capture parental views on caregiving. Measures included satisfaction or the amount of pleasure derived from parenting, communication. or parent's perceptions of the effectiveness of their communication. Beurkens et al. (2013) found an increased severity of autism impaired parent-child interactions. Child communication and social interaction abilities predicted outcomes on parent-child interaction measures. However, severity of child autism features had no effects on 
interpersonal relatedness between child and parent. While the social and communication deficits interfered with parent-child dyadic interplays, parents' feelings about their children and quality ratings of their relationship with their child did not differ from parents of children who were typically developing. Other research supported this finding. Hoffman, Sweeney, Hodge, Lopez-Wagner, and Looney (2009) found attachment was intact between parents and infants with autism. This study reported that parents of infants with autism felt as close to their babies as parents of typically developing children.

\section{Parent experiences with autism during the lag time between onset and} services. DePape and Lindsay (2015) conducted a metasynthesis of qualitative research on parenting experiences caring for a child with autism. The study searched peer reviewed journal articles using subject headings related to autism, parenting, sociological concepts, qualitative research, and phenomenology. After applying inclusion criteria 31 studies were selected. Using constant comparison analysis six themes emerged from the literature: (a) prediagnosis, (b) diagnosis, (c) family life, (d) adjustment, (e) navigating the system, (f) parental empowerment, and (g) moving forward. The study used a Family-Life Cycle Theoretical Framework (FLC) (Author/Date) and in doing so identified themes that spanned parenting during and after infant / toddler years. By using a FLC, the study offered insight into an evolution of caregiving and early intervention experiences from the parent's point of view. Several themes from DePape and Lindsay's study are particularly relevant to understanding caring for infants and toddlers with autism in the context of service delivery experiences. Results from the metasynthesis offered insight into caring for infant and toddlers with autism during the lag time between 
AOR and service commencement.

During the pre-diagnosis phase some parents in DePape and Lindsay's study reported that their infants displayed typical development and remembered celebrating typical milestones. Other parents felt there was something atypical about their child's early development. Some parents associated these concerns with autism behaviors (e.g., not responding to his name, delayed verbal communication). Many parents referenced their child's behavior against other children and received feedback from neighbors and friends.

DePape and Lindsay found that once parents recognized concerns they began searching for answers. After receiving a diagnosis parents expressed both relief and devastation. Some parents reported having mixed feelings. During the initial stages of diagnosis parents felt feelings of guilt and blame, looking for causes and explanations for their child's autism.

Based on the analysis of parenting themes in caregiving a child with autism, DePape and Lindsay (2015) made recommendations for improving services. More information from service providers about early autism would help parents recognize autism markers in their infants. Based on qualitative reports on adapting interventions, the researchers recommend incorporating culturally relevant activities into the interventions. During the advocacy phase parents learned all they could about autism and voiced concerns when they disagreed with service providers. The role of parent advocate was challenging to authority figures in the medical model such as doctors who historically have been gatekeepers of knowledge. 
Parent studies focusing on caring for older children with autism. The review of the research literature for this study found relatively few qualitative studies investigating caregiving and the lived experiences of parents of children with autism (see Corcoran et al., 2015). Most published studies involved parents of older children with autism (i.e., ages three and up). The majority of caregiving and autism studies examined through this review were limited to mothers' experiences with most studies investigating caregiving experiences of middle-class, Caucasian families with children with autism. In an effort to garner insight into lived experiences of parents for infants and toddlers with autism, the parameters for the caregiving literature search in this study were expanded to include older children, retrospective accounts, and international studies. Four themes emerged from the review of the research literature for this section: (a) accessing services, (b) feelings of inadequacy and stigmatization, (c) impact on family, and (d) adaptation.

Parents wanted more information. Lived experiences of parents of infants/toddlers with autism begin at the first concern by parents about their child's development (Osborne \& Reed, 2008). As parents of children with autism begin to navigate the system of care, they may encounter issues that promote or hinder positive associations with health care and social service practitioners (Meirsschaut, Roeyers \& Warreyn, 2010; Osborne \& Reed, 2008). Osborne and Reed (2008) conducted a focus group study with three groups of parents from southeast England. The groups were comprised of parents with (a) preschool-aged children, (b) primary age children, and (c) secondary-age children. Parents were excluded if their child received a diagnosis less than six months prior. The parents of preschool age children recently diagnosed with 
autism overwhelmingly reported wanting as much information about autism up front even when they recognized it might be overwhelming or difficult to absorb. Parents in Osborne and Reed's (2008) study also reported dissatisfaction with the speed of diagnosis, "the diagnosis comes too late" (p. 217). Other suggested improvements included information on autism and what to expect, standardization and speed of diagnostic process, and information about organizations and services. Eighty-four percent of preschool parents stated that autism information should have been given immediately after diagnosis. When asked what services they wanted available, $40 \%$ of parents of preschool aged children with autism wanted ABA type treatment packages and 20\% wanted educational help from specialists, teachers, or an early childhood program placement. Parents expressed frustration and dissatisfaction with speed of services, information available, and inconsistent practices.

Parents' frustration with services. Corcoran and colleagues (2015) conducted a systematic review and meta-synthesis of lived-experience research caregiving for children with autism. The researchers did not indicate the children's ages. Several themes emerged from the data. Parents reported an array of feelings surrounding their experiences with service providers for their children with autism. Consistent with other research in the field, parents often experienced stress and frustration when accessing and navigating services (Brogan \& Knusse, 2003; Goin-Kochel, Mackintosh, \& Meyers, 2006). Some sources of frustration related to the process of diagnosis and availability of services. Parents reported difficulty navigating services and overwhelmed and lost with the quantity of service options and time commitment. They desired one route for 
accessing information. Some participants reported that once service needs were identified they experienced difficulty accessing services and a lack of availability of services. Participants also reported on experiences with service providers. They felt there was often little direction and support from providers, a lack of patience from some professionals, and some participants felt pediatricians and specialists minimized their complaints.

Autism as the center of the family. Hoogsteen and Woodgate (2013) conducted a phenomenological study of lived experiences of 28 families living in rural communities. The researchers summarized the parent's lived experience as "making the invisibility of autism visible" (p. 136). Parents reported that once their child was diagnosed with autism, the disability became the center and focus of family life. Parents felt they filled multiple roles (e.g., parent, advocate, organizer, tutors, and problem solvers). Many parents reported exhaustion, fatigue, and burnout. Because they were isolated geographically, these feelings were exacerbated because parents felt they had no one to turn to who truly understood their experiences.

Impact on family. Parents expressed feelings of exhaustion and frustration in managing the number of doctor's appointments and services for their children. This was reported in most of the qualitative investigations of caring for a child with autism (see Corcoran et al., 2015). Keeping appointments required an extraordinary amount of time. Hours of therapy, travel time, and paperwork also had implications on parent ability to earn an income thus compounding the problems associated with the high costs of raising a child with autism (Phelps, Hodgson, McCammon, \& Lamson, 2009). 
Positive experiences as parents of a child with autism. Parents also had positive associations with having a family that included a child with autism. They reported learning to appreciate life in a different way and seeing things in ways that parents of children who were typically developing could not (Hunt-Jackson, 2007; Lutz, Patterson, \& Klein, 2012). Many parents described their children as a joy and despite the difficulty and stigmatization; they would not change things (e.g., Corcoran et al., 2015; Hutton \& Caron, 2015).

Some research groups, particularly those from the disability rights perspective, challenged research focusing on grief and stress associated with parenting a child with autism. Ryan and Runswick-Cole (2008) describe a no win situation experienced by mothers of children with autism. This group --by default-are either devalued because of their child's disability or assumed to be grief stricken because of it. Both scenarios reduce the complex experience of parenting a child with a disability to homogeneous and narrowly socially constructed. However, even dominant paradigm research reported a complex and nuanced experience of mothering a child with autism. Nelson (2002) conducted a metasynthesis of qualitative studies on mothering children with disabilities. While Nelson reported stress and increased intensity in the mother/child relationship in parenting a child with a disability, mothers also placed great value on their relationship with their child and found parenting rewarding. The meta-synthesis revealed that while grief, stress, and fear were present, those feelings were often associated with the initial phase of adjusting to the child's diagnosis. In a mixed-method study, Green (2007) found mothers were satisfied with their relationship with their children with disabilities, but felt 
burdened by socio-cultural constraints. Disability rights groups challenged a circumscribed and largely pathological view of parenting a child with a disability. Greif associated with having a child with autism may reflect the attitudes about normalcy more than the authentic experience partnering a child with autism. Alternatively, parental grief may reflect internalized stigma associated with disability and not loss of having a "normal" child. Ryan and Runswick-Cole (2008) argued, "Dominant discourses do not allow us to see beyond this position of negativity, and there is little engagement with the other dimensions of mothering a disabled child."

Feelings of stigmatization. A number of qualitative studies of caregiving with older children with autism reported that having a child with autism could be a stigmatizing experience. Some studies found that parents engaged in strategies to inform others about their child's condition, both to inform service providers but also to increase understanding by others in their daily lives (Hoogsteen \& Woodgate, 2012; Meirsschaut et al., 2010; Neely-Barnes, Hall, Roberts, \& Graff, 2001). Lutz et al. (2012) reported that some parents felt the negative reaction from others caused a sense of discomfort while other parents used judgments and negative interactions as an opportunity to advocate for their child so that he/she could feel like a part of society.

\section{Section Summary}

The literature reviewed on caring for infants, toddlers, and children with autism resulted in several themes. There was little research with children under age three. Parent recognition of symptoms research provided insight into the gap in services, a time when parents were without support during the first three years of their children's lives. 
Parent/infant interaction research with infants and toddlers with autism shed light on the impact of infant social-communication deficits on early parent/infant interactions. Despite disruptions in dyadic functioning, research indicated no negative effects on attachment and quality of relationship between parents and their infants with autism.

A number of themes emerged within the research with older children with autism. Parents expressed frustration over the amount of time and energy required in accessing services, appointments, and other caregiving activities associated with their child's autism. Parents also reported feelings of stigmatization and inadequacy. Despite the hardships reported in families with an infant with autism, research indicated parents appreciated their children for whom they were and derived joy from the experience.

\section{Review of Research Literature on Autism EI and IDEA, Part C}

\section{What is IDEA Part C?}

The Individuals with Disabilities Act, Part C. In 1987, the federal government recognized an urgent and substantial need for educational intervention services for infants and toddlers (birth to age three) with disabilities. As a result, Part $\mathrm{C}$ was added to the Individuals with Disability Education Act (IDEA, 1997). Prior to 1987, IDEA addressed the special education needs of children between three and twenty-one with no provisions for infants and toddlers (i.e., birth through age 2). However, in light of evidence regarding the powerful impact of intervening before age three on child developmental outcomes, IDEA Part C was adopted and made special education services available for infants and toddlers. As a result, the federal government offered monetary incentives to states providing special education EI programs (IDEA, 2004). As of this review, all states 
participate in IDEA, Part C (Early Intervention, 2015).

Individuals with Disabilities Education Act, Part C outlined requirements for Individualized Family Service Plans (IFSPs) to meet the developmental needs for infants and toddlers with disabilities. The cornerstone of the IFSP is family-centered planning in which parents are equal partners with interdisciplinary teams in the development and implementation of IFSPs.

Individuals with Disabilities Education Act, Part C was strongly informed by best practices in EI (BP-EI). The purpose of IDEA, Part C is to (a) enhance the development of infants and toddlers with disabilities, (b) reduce educational costs by minimizing the need for later special education through EI, (c) minimize the likelihood of institutionalization, and maximize independent living, and (d) enhance the capacity of families to meet their child's needs (Early Intervention, 2016; IDEA, 2004). Unlike earlier clinician led practices, the guiding principle for IDEA, Part C was the level of parent control in their child's EI planning and implementation. Individuals with Disabilities Education Act, Part C required the development of IFSPs for all infants and toddlers with disabilities. Individuals with Disabilities Education Act, Part C outlined the IFSP process as:

[The IFSP] contains information about the services necessary to facilitate a child's development and enhance the family's capacity to facilitate the child's development. Through the IFSP process, family members and service providers work as a team to plan, implement, and evaluate services specific to the family's concerns, priorities, and available resources. A service coordinator then helps the family by coordinating the services outlined in the IFSP. (Early Intervention, 2016, (n.p.)

Definition of family centered practices. Murphy, Lee, Turnbull, and Turnbull 
(1995) outlined the essential ingredients for effective EI according to BP-EI (a) include families in decision-making, planning, assessment, and service delivery at family, agency, and system levels; (b) develop services for the whole family and not just the child; (c) insure plans are guided by families' priorities for goals and services; and (d) respect families' choices regarding the level of their participation (Murphy et al., 1995). The cornerstone of family centered practice is treating families with dignity and respect. Consistent with Bronfenbrenner's (1979) Ecological Systems Theory, EI needs to be sensitive to the diversity of families including cultural and socio-economic differences. When professionals are sensitive to family perspectives they provide choices, support families in decision-making, and provide resources. By engaging in these help giving practices, early interventionists can empower families and increase parental capacity to enhance their children's' development (Bruder, 2000).

Impact of family-centered practices in child outcomes. Best Practices in EI research linked family-centered practices with infant/toddler developmental outcomes. When parents felt supported and respected, they were committed to following the service plan (Dunst \& Trivette, 1989; Dunst et al., 1994; Noon \& McCormick, 1993). When parents correctly practiced goals with their infant/toddler, child developmental outcomes improved. When goals were aligned with family culture and parent priorities, parents were more likely to meet the intensive practice schedule required for maximum gains and promotion of generalization of new skills in infants/toddlers with disabilities (Bernheimer \& Keogh, 1995). Best practices in EI research repeatedly demonstrated that with collaborative support, parent involvement increased intervention effects (Ketelaar, 
Vermeer, Helders, \& Hart, 1998; Shonkoff \& Hauser-Cram, 1987). There were also correlations between the level of support families received and intervention effects (Mahoney, Boyce, Fewell, Spiker, \& Wheeden, 1998). Bernheimer and Koegh (1995) argued that parents failed to implement and sustain implementation when strategies did not fit daily activities of the family or if the strategy was not compatible with the parents' values, beliefs, or goals. Other studies found that building the capacity of the parents in the home environment enhanced child development outcomes (Peterson et al., 2007; Trivette, Dunst, \& Hamby, 2010).

\section{Autism and IDEA, Part C}

Overview. Given the lag time between onset of autism and intervention, most infants and toddlers with autism and their families do not receive treatment. By the time children are diagnosed with autism, they have aged out of EI services under IDEA, Part C. Instead, they are eligible for early childhood special education services (see IDEA, Part B). Early childhood special education practices (ECSE) for this age group is very different methodologically from EI. Early childhood special education services are most often child-centered, delivered in a school or clinic setting, and do not directly involve the family in planning and treatment (USDOE, 2005). As the numbers of infants/toddlers receiving a diagnosis of autism increases, there is a greater need for AUT-EI.

While first-generation AUT-EI research was on the rise, there was also growing concern over the incongruence of these models with Part C (Dawson, 2008; Rogers \& Vismara, 2014; Zwaigenbaum et al., 2005). Rogers and Vismara (2014) reviewed the first generation AUT-EI studies and examined the incongruence between AUT-EI treatment 
models and IDEA, Part C regulations. Rogers and Vismara found (a) 48\% of AUT-EI studies did not use practices to support parent/child interaction, (b) $41 \%$ did not use strategies to build family capacity to support child skill acquisition, and (c) $63 \%$ implemented interventions outside of natural family or community. Rogers and Vismara's findings highlighted the growing consensus among experts and researchers in the field that AUT-EI treatment assumptions and designs need re-examination (Camarata, 2014; Dawson, 2005; Warren et al., 2011).

Early autism screening and identification. In recent years, there were strategic screening efforts aimed at identifying infants who might be displaying early signs of autism. The American Academy of Pediatrics recommended early surveillance during well-check baby visits beginning at nine months. At minimum, pediatricians were instructed to screen for autism at 18 and 24 months (Johnson \& Myers, 2007). These efforts were aimed at identifying infants/toddlers at risk for autism as early as possible so they could begin treatment to ameliorate the effects on development. The gold standard diagnostic protocol for autism in young children is a combination of assessments, observations, and clinical opinion.

IDEA, Part C services for infants/toddlers with autism. Stahmer and Mandell (2007) conducted a survey of state EI for infants and toddlers with autism from identification to treatment. It was one of the few studies examining IDEA, Part C programs and autism. Stahmer and Mandell found inconsistencies among U.S. states in implementation of IDEA, Part C for infants and toddlers with autism. The states varied widely in identification practices, standards for professional qualifications. Diagnostic 
criteria for eligibility varied considerably depending on the state special education program. Sixty-five percent of states reported that they did not offer autism-specific services for infants and toddlers. Forty percent of the states without autism specific programs stated they provided individualized treatment to meet the unique needs of each child. Most states with reported autism-specific programs employed eclectic models using a combination of recommended treatments based on the unique needs of the child. Many treatment approaches had a strong evidence base with preschool age children with autism (e.g., Early Start Denver Model (Roger \& Dawson, 2010), SCERTS method (Prizant et al., 2003), Treatment and Education of Autistic and related Communicationhandicapped Children (TEACCH; Mesibov, Shea, \& Schopler, 2005) incidental teaching (Warren \& Kaiser, 1986). However, there was little evidence-base for these models with infants and toddlers.

\section{Section Summary}

Individuals with Disabilities Education Act, Part C outlines requirements for providing EI services for all infants and toddlers with special needs. These services, although individualized to meet the developmental needs of the child, require a familycentered approach and implementation in natural contexts. To date, there is a dearth of efficacy research for AUT-EI that meets IDEA, Part C requirements. Most states do not provide autism specific EI services and rely instead on eclectic, individualized approaches. There is a need for effective AUT-EI treatment models that meet IDEA, Part $\mathrm{C}$ requirements. At the time of this literature review, there are few studies examining this issue. 
Review of Research Literature on Family Perspectives, Values, Priorities, Resources Introduction

As discussed earlier, parent perspectives play a central role in the design of IFSPs for infants and toddlers with special needs. Parent perspectives about caregiving are influenced by a myriad of nuanced and practical realities of daily lived experiences. Bronfenbrenner's (1979) Ecological Systems theory is particularly relevant to parent perspectives in AUT-EI research. Issues such as socioeconomic status, family support, culture, infant's health, parent's health all contribute to the unique perspectives of each parent. Few studies within the AUT-EI literature investigated parent perspectives specifically focused on caregiving for an infant/toddler with autism. Autism EI research had a strong quantitative representation. Experimental design studies predominated the literature. To address the lack of AUT-EI parent perspective literature, this review was expanded beyond the study parameters. In addition to qualitative parent perspective research in the AUT-EI literature, this review included other EI studies and qualitative research related to parenting older children with autism. Two themes emerged from this expanded review of research literature, (1) parent satisfaction with services and (2) mental health issues associated with caring for an infant/toddler with autism. These themes are discussed in the subsections that follow.

\section{Parent Satisfaction with Services}

Parent perspectives on the relationship with interventionist. A number of studies reported that parents working with early interventionists felt satisfied when these individuals were nonjudgmental, sincere, caring, creative, supportive, and responsive 
(Applequist \& Bailey, 2000; Bailey, Scarborough, \& Hebbeler, 2003; Hurtubise \& Carpenter, 2011; Jackson, Traub, \& Turnbull, 2008; Wade, Mildon, \& Matthews, 2007). Parents sometimes reported frustration with the lack of consistency among professionals when determining the most effective intervention approach. There was also frustration when service providers seemed inflexible, unorganized, unknowledgeable, unreliable, and unresponsive to individual families' informational, support, and emotional needs (Grindle et al., 2009; Patterson \& Smith, 2011; Rodger et al., 2008; Webster et al., 2004). Parents reported dissatisfaction when interventionists failed to provide direction and support (Kalash \& Olson, 2012). When EI services were delivered in an unsupportive, non-individualized manner, families reported negative experiences with interventions that felt invasive and disruptive, resulting in increased family stress. Parents became overwhelmed, isolated, and incapable of providing intervention (Beals, 2004; Grindle et al., 2009; Patterson \& Smith, 2011). Some parents of children with autism reported often feeling that they were not listened to when first reporting concerns about their child's atypical development. Parents and professionals often had differing opinions about the needs of children with autism, as well as about strategies and outcomes even within a family-centered service model (Carbone, Behl, Azor, \& Murphy, 2010; Dillenburger, Keenan, Doherty, Byrne, \& Gallagher, 2010; Sperry, Whaley, Shaw, \& Brame, 1999). Parents felt supported when they were involved in decision-making and collaboration with the EI team (Beals, 2004; Patterson \& Smith, 2011; Webster et al., 2004). Parent satisfaction with AUT-EI was associated with effective interactions with service provider (e.g., expressing compassion, effective listening, communicating in a supportive, non- 
judgmental manner, cultural sensitivity, and building on family strengths (e.g., Applequist \& Bailey, 2000; Coogle et al., 2013)

Effects of early interventionist behaviors and attitudes on parent satisfaction may be robust across cultures. Applequist and Bailey (2000) interviewed 52 Navajo parents of young children receiving EI services. On average, the families had been receiving services for 15 months and received home visits twice per week. The researchers used an interview protocol developed for the study that came from a composite of several client satisfaction questionnaires. Applequist and Bailey reported that most Navajo families receiving EI services were satisfied. The study also reported that program characteristics, not provider demographics (e.g., providers of same cultural background) predicted satisfaction. One of the strongest predictors of satisfaction with EI was how much the parents perceived the programs to be family-centered. The researchers used the Family Orientation of Community and Agency Services measure (FOCAS, Bailey, 1991) to determine parental perception of family-centered services. Questions on FOCAS centered on family participation, program philosophy, and family needs. A discrepancy between a typical experience and ideal experience was used to determine how participants perceived family-centeredness.

Services that enhance family quality of life. Epley, Summers, and Turnbull (2011) conducted a survey of families receiving EI services through IDEA, Part C. Seventy-seven families responded to a quality of life survey through an anonymous web link. The parent responses to the survey were primarily positive. However, satisfaction with EI services predicted early childhood and family outcomes. Further, the more EI 
services met families' perceived needs, the greater the reported family quality of life. The child and family outcomes were defined as: (a) families know their rights and advocate effectively for their child, (b) families understand their child's strengths, abilities, and special needs, (c) families help their child develop and learn, (d) families have support systems, and (e) families are able to gain access to desired services and activities in their community (Epley, Summers, \& Turnbull, 2011).

Parent's desire for involvement. Parents of infants/toddlers with disabilities including autism showed a strong desire for involvement in interventions (Applequist \& Bailey, 2000). They expressed a desire to be more involved and reported uncertainty regarding their role in developing IFSP options (Jackson et al., 2008). Parents of young children with autism valued being included in decision-making, having access to resources, receiving services in the home, allowing for flexibility in services, and utilizing a team approach (Beals, 2004; Patterson \& Smith, 2011; Webster et al., 2004). Parents reported valuing opportunities to establish social networks and relationships with other families of young children with autism (Grindle et al., 2009) and to learn new intervention strategies that could improve the child's communication, play, and social skills (Grindle et al., 2009; Patterson \& Smith, 2011; Webster et al., 2004).

Parent perspectives on quality of services. A number of qualitative investigations found that parents had practical concerns over services. These concerns translated into an overall perception of service quality. Parents were concerned about cost of service delivery. They reported frustration with the length of wait-time between EI referral/assessment and determination of edibility/service delivery (Bailey et al., 2003; 
Bailey et al., 2004; Grindle et al., 2009; Haring, Lovett \& David, 2001; Shannon, 2004;

Wade et al., 2007; Webster et al., 2004). Practical matters of service delivery also factored into parents' reports of satisfaction. Some parents expressed that services should be flexible so they could find a convenient time and location (Coogle et al., 2013).

Parents reported dissatisfaction in the absence of social supports and service options (Jackson et al., 2008). Families also desired services that resulted in explicit, practical, individualized, and child-friendly interventions based on identified learning goals for the child (Beals, 2004; Grindle et al., 2009; Patterson \& Smith, 2011; Rodger et al., 2008; Webster et al., 2004). A recent study of parent perspectives on intervention services for infants/toddlers with autism found that increased services and resources resulted in greater child outcomes (Bradshaw et al., 2015). Parents of infants/toddlers with autism viewed EI as effective when they were provided information about autism, child development, and community services and resources (Cridland et al., 2014).

Section summary. The emerging qualitative AUT-EI research supported the need for a richer understanding of the myriad of issues associated with parenting a child with or at risk for autism (Cridland et al., 2014; Seligman \& Darling, 2007). Existing research about parent-perspectives within AUT-EI literature and related research underscored the importance of positive relationships with interventionists, collaboration on service plans, and access to information and resources. These themes were evident across most studies and were linked to child development outcomes. These findings were consistent with best practices in early intervention and the theoretical frameworks for this study.

\section{Parent Mental Health and Parenting Infants/Toddlers with Autism}


The second theme within the parent perspective literature was the effects of stress in parents of an infant/toddler with autism. There was an abundance of research related to stress and mental health problems associated with parenting a young child with autism (e.g., Estes et al. 2009; Griffith, Hastings, Nash, \& Hill, 2010; Hamlyn-Wright et al. 2007; Hoffman, Sweeney, Hodge, Wagner, \& Looney, 2009; Kasari \& Sigman, 1997). This stress was found to be greater in parents of infants/toddlers with autism than those with TD or DD (e.g., Estes et al., 2009; Griffith et al., 2010). It was also reported that parents of infants, toddlers, and young children with autism experienced more mental health issues than those with young children with TD or DD (Baker-Ericzén, BrookmanFrazee, \& Stahmer, 2005; Kasari \& Sigman, 1997; Schieve, Blumberg, Rice, Visser, \& Boyle, 2007; Yamada et al., 2007).

Effects of infant autism on parent stress. A child's autism may have implications for parental stress particularly when behavior and development are greatly impacted. Stuart \& McGrew (2009) reported that child autism severity negatively impacted parental physical and mental health and closeness and attachment between mother and child. In general, the greater the severity the more strained the family relationships became (Stuart \& McGrew, 2009). Significant delays in infant social behavior and self-regulation behavior (e.g., sleeping, eating, and emotional regulation) also increased parental stress (Davis \& Carter, 2008). Phelps, McCammon, and Weundsch (2009) conducted surveys with 80 parents of children with autism. Stress of caregiving for a child with autism correlated with some measures of overall enrichment and quality of life. Based on the results, three researchers made recommendations for EI 
practices that accounted for parent stress and increased parent self-confidence and selfefficacy through stress-informed support and information.

This literature review yielded few studies investigating ecological factors influencing stress in parents with children with autism (e.g., feelings of stigmatization, and lack of support). Osborne, McHugh, Saunders, and Reed (2008) proposed that life conditions likely moderated parental stress (e.g., fatigue, intensity of intervention, and competing priorities associated with family life). Most of the AUT-EI research linked parent stress with infant disability factors (e.g., symptom severity or behavioral problems) or parent responses to their child's condition (e.g., grief). Hayes and Watson (2013) conducted a meta-analysis of parent stress studies and reported that parents of children with autism had much greater overall stress than parents of children who were typically developing and parents of children with developmental delays. This finding suggests that factors associated with having a child with autism were inherently more stressful.

Within the larger body of BP-EI research, other influences on parenting correlated with stress and parental mental health problems. Social support stood out as a mediator of stress associated with parenting a child with a disability (Dunst, Trivette \& Hamby, 2007). Parental perspectives (e.g., view of autism and goals for their child) also can moderate family stress (Cunningham, 1987; Marfo, 1991). The BP-EI research offered insight into the transactional effects of stress, parenting, and child progress. Research also demonstrated that as family risk factors increased, child outcomes declined (Sameroff et al., 1993). 
Section summary. Two themes emerged from the parent perspective literature (a) perspectives on services and (b) parent mental health associated with parenting an infant/toddler with autism. Service experiences influenced parent perspectives on AUTEI for their young child. A major trend was the importance of a positive relationship between parent and interventionist. Parents reported positive experiences when providers listened, treated them with respect, and provided information. Wait time for diagnosis, cost of services, and availability of resources also influenced parent perspectives.

Parenting an infant with autism was associated with increased stress. Many quantitative and qualitative studies reported on the stress associated with caring for an infant/toddler with autism. Parenting a young child with autism was associated with more stress than parenting a child with typical development or developmental delay. There were no studies within the birth to three years of age parameters that discussed the effects of caring for an undiagnosed infant while waiting for diagnosis. Few studies explored the effect of ecological influences as a moderator of parenting stress and caring for an infant/toddler with autism. Most research was limited to parental feelings about their child's diagnosis, behavior, or service needs. There was a dearth of research exploring perspectives on parenting an infant or toddler with autism.

\section{Summary of Lived-Experience Literature Review}

Four themes emerged from the AUT-EI research literature review (a) What is AUT-EI?, (b) IDEA, Part C and AUT-EI, (c) Caring for an infant/toddler with autism, and (d) Perspectives of parents of infants/toddlers with autism. Figure 4 summarizes the trends within these four larger themes. 


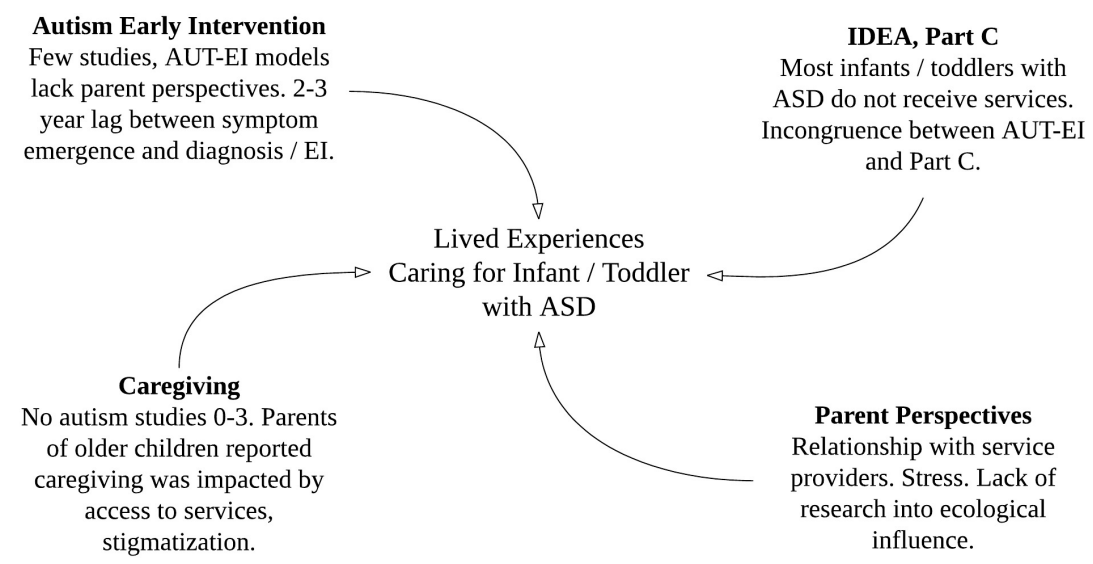

Figure 4. Themes found in the AUT-EI literature.

\section{Review of the Methodological Literature}

\section{Phenomenological Research Methodology}

Descriptive phenomenology. The methodological design for this study is discussed in more detail in Chapter 3. In this section, I offer an introduction to the methodology and appropriateness of the study. In order to investigate my research questions. Towards this end, I selected Colazzi's (1978) descriptive method, a subset of Husserl's (1970) phenomenological approach. Phenomenological research was an appropriate choice given the emphasis of my inquiry on re-examining the things "we tend to assume we know; things we think we have settled" (Vagle, 2014, p. 56). The lived experiences of parents of young children with autism remains an area of research with few investigations to date. I conducted two interviews with each participant to explore the lived experiences of parents of young children with autism. The first interview was an unstructured, family story conversation guided by family photographs (i.e., photo 
elicitation method) and written prompts. The second interview built on the conversations from the first interview and was guided by prompts. My study employed descriptive phenomenological and photo-elicitation methods. I review these methodologies in the following sections.

Colaizzi's (1978) approach is a subset of descriptive phenomenology (Husserl, 1970) and falls under the qualitative research paradigm. Qualitative research is an appropriate choice when the focus of investigation is on gathering fine-grained data to understand unique experiences (Merriam, 2009). The underlying assumption of all qualitative traditions is that meaning is socially constructed as a person interacts with the world (Merriam, 2009). The qualitative research process involves the researcher's active participation in both data collection and analysis. Descriptive phenomenology is an interpretive, open, flexible, and responsive process allowing the researcher to deeply and thoughtfully settle into and sit with the phenomenon under investigation (Vagle, 2014). This process is reflective, one in which the researcher deeply contemplates the concrete ways in which phenomena are lived (i.e., in this study, caregiving an infant/toddler with autism) and not how they are represented or conceptualized.

Phenomenological investigations allow for deep exploration of research questions and a means to gather the evidence necessary to capture both individual lived experiences and the essences of the phenomena of these lived experiences. Descriptive phenomenology, unlike other branches of phenomenology (e.g., hermeneutic, existential, and generative historicist), emphasize describing human lived experiences to describe universal essences (Wojnar \& Swanson \& Schonwald, 1988). Essence is a concept in 
phenomenological research referring to universal human constructs derived from collective lived-experience phenomena.

Phenomenological research studies often use a number of possible sources of data. These range from standard research techniques (e.g., interviews and observation) to creative forms (e.g., drawing, collage, and creative writing; Vagle, 2014). A key principle underlying phenomenological data gathering is a researcher's adherence to an open mindset. The researcher is embedded in the research process and through reflection, conceptualization, and engagement with participants. Data gathering methods are rooted in the phenomenon under investigation. An important concept in descriptive phenomenological research is bracketing, which is an investigator's attempt to remove preconceptions about the phenomena under investigation (Wojnar \& Swanson, 2007). Phenomenology adheres to ideological assumptions that investigators have preconceived notions, biases, and opinions likely influence their interpretation of qualitative data. Colaizzi's (1978) method includes strategies for bracketing preconceptions and bias, an important aspect of phenomenological research. Given that the goal of the present study is to understand parents and their daily lived experiences of parents of an infant/toddler with autism; Colaizzi's method is very appropriate. Colizzi's (1978) descriptive method involves a 7-step framework for data analysis and interpretation (Wojnar \& Swanson, 2007). This framework will be discussed in detail in Chapter 3.

\section{Limitations of phenomenological methodology.}

Validity. Validity is of concern in all qualitative traditions. Lincoln and Guba (1985) described and defined four concepts of validity in qualitative research (1) 
trustworthiness is confidence in the 'truth' of the findings, (2) transferability is showing that the findings are applicable to other contexts, (3) dependability shows that findings are consistent and could be replicated, and (4) confirmability is neutrality and the extent to which the findings are shaped by participants not researcher interests or a priori assumptions. Colaizzi's (1978) data analysis method addresses the four concerns associated with qualitative research validity. This will be discussed further in Chapter 3 .

Time and quantity of data. Another limitation with all qualitative methodologies and, phenomenology in particular, is the length of the data collection process and the copious amounts of narrative data generated. Lester (1999) pointed out that while the depth of inquiry lends itself to rich, fine-grained data from which perspectives could be inferred, some participants may not be comfortable with the time or emotional commitment of the process. As a result, many researchers have difficulty with the large quality of qualitative data generated (e.g., interview notes, audio recordings, observation notes, and diaries). Analysis can also be messy with many ways of linking different portions of discussions. (Lester, 1999).

Sample size. Consistent with other qualitative traditions, phenomenology focuses on a limited number of participants (i.e., small sample size). One of the main limitations with smaller sample sizes is a lack of generalizability (Merriam, 2009). A larger sample size would have been inconsistent with answering the research questions of this study. To investigate the experience of parenting an infant/toddler with autism more deeply, fewer participants, and qualitative data collection was most appropriate.

Summary of limitations. The strength of phenomenological research is its 
usefulness in understanding experiences that are often overlooked by traditional research paradigms. By definition, phenomenological methodology is concerned with subjective, lived-experience when investigating a phenomenon. As a result, small sample size, unstructured data collection, reflective researcher practices, and bracketing are the predominant practices in data collection and analysis. Phenomenological research was a good fit for my study and the benefits of this methodology outweighed the limitations. There is an unfortunate history of marginalization and stigmatization of parents of infants/toddlers with autism and their voices remain minimally present in the predominant research literature. Phenomenological methodology addressed this problem in a way that quantitative or more structured research methods could not.

Phenomenological Literature Review. The literature review in this chapter yielded very few AUT-EI qualitative studies of parent perspectives and no phenomenological studies of the lived experiences of parents of infants/toddlers with autism. However, the utility of phenomenological research in informing clinical practice has a precedent in the health care fields. Of particular relevance was the research in the field of nursing (Beck, 1994). In the following paragraphs, I describe phenomenological studies conducted in neonatal nursing. These examples will further support the use of this methodology in investigating the lived experiences of parents of infants/toddlers with autism.

There are similarities between the parents with a child recently diagnosed with autism and those with a premature infant or one in critical care. Parent adjustment to the situation may cause great stress (Bergström, Wallin, Thomson, \& Flacking, 2012; 
Lefkowitz, Baxt, Evans, 2010; Watson 2011). Stress associated with parenting and autism was well represented in the literature. In neonatal family-centered services, a stated goal was to facilitate infant/parent bonding (e.g., McGrath, 2001, Mok \& Leung, 2006, Fegran et al., 2008). Similarly, AUT-EI treatment objectives involved increasing and enhancing parent/infant interactions to minimize the effects of social-communication disruptions associated with autism in infancy. Because phenomenological research aims to gain insight and understanding of lived-experience at a deep and personal level, it was well represented in the field of neonatal nursing. Rich and nuanced themes emerged from the phenomenological research data. These findings then informed nursing practices with parents and their infants. Neonatal healthcare phenomenological studies reported that parents experienced difficulties during a period of adjustment as they assumed responsibility for their care of the infant. This transition often required additional support from the in-home nursing staff (Murdoch \& Franck, 2012; Nicolaou, Rosewell, Marlow, \& Glazenbrook, 2009; Rehm \& Bisgaard, 2008).

Dellenmark-Blom and Wigert (2014) conducted a phenomenological study with 200 parents transitioning from the Neonatal Intensive Care Unit (NICU) to home care. The study employed using the reflective lifeworld approach (Dahlbert et al., 2008). The lifeworld approach employs phenomenological methodology to understand the lived experiences of everyday phenomena. Through shared narratives and interviews, rich and nuanced insights emerged about parents' thoughts, emotions, and activities. The study found that parents felt insignificant when they brought their babies home after the NICU. When nurses spent time in conversations about parents' feelings, parents reported 
perceiving themselves with increased significance to the nurse and infant. A repeated theme in Dellenmark-Blom and Weigert's study was the role of nurses in empathetic support to parents as they assumed responsibility for their child's care. This responsibility included adjustment to their child's healthcare requirements, changes in the family routine, and emotional feelings. Parents' experiences with a baby coming home for the first time from the NICU, was an inner emotional journey. As infants moved from the NICU to home, parents began to perceive themselves as parents of their own infant. This theme related to the concept of self-efficacy in parenting an infant and toddler with disabilities. Parent self-efficacy is linked to intervention success. Dellenmark-Blom and Weigert's phenomenological study illustrated that neonatal home care must be structured around parents needs following discharge from the NICU and not simply the medical needs of the baby.

Landgren and Hallström (2011) conducted a phenomenological hermeneutic study of parents' experiences of living with a baby with infantile colic. The study found colic overshadows everything. Qualitative investigations of parenting a child with autism reported similar findings (Beals, 2004; Grindle et al., 2009; Patterson \& Smith, 2011). Landren and Hallström used phenomenological methods for interviewing and analysis with 25 parents of babies with colic. At the start of the interview, parents were asked to share their experiences of having a baby with colic. Participant answers were followed up by the researchers with questions that encouraged elaboration on the lived experience (e.g., "How did you feel then?" "What did you mean?"). After transcription, the researchers read the participant responses and formulated a naive understanding of the 
lived experiences. A structural analysis was then conducted by dividing the text into meaning units and themes and subthemes were generated. The authors reviewed the original text and analyzed meaning using their own imaginations/associations, the literature, and the themes generated during the analysis. Upon analysis, the authors used several metaphors to describe the experience of parenting an infant with colic (e.g., journey on a stormy sea, keeping a stiff upper lip, tried every trick, life world filled with loneliness, and powerlessness). These results were used to inform nursing practice. The stormy sea metaphor helped nurses find new ways to help parents cope during this difficult time. By reassuring parents that colic would not harm the baby and the situation would improve, the researchers hypothesized that a parental sense of hopelessness could be alleviated and attachment between parent and baby preserved.

\section{Photo-Elicitation Interview (PEI) Studies.}

There were no studies found in the AUT-EI literature that employed PEI methodology. Photo-elicitation research was reportedly used to augment qualitative interviewing in other areas of research. Clark-Ibanez (2004) used PEI to augment ethnographic research examining academic success in urban schools in Los Angeles. Data collected through PEI generated participant responses that went beyond the scope of interviews using words alone. Clark-Ibanez reported that while the images in the photographs may not contain new information, they might have triggered meaning that would have otherwise been missed. In one interview, Clark-Ibanez discussed a participant's photographs of her kitten. Once the data had been collected, it was apparent that the content of the photographs were not important. Instead, the participant described 
how her parents had given her the kitten when she moved to a Watts neighborhood and started a new school. The kitten represented comfort in the face of losing friends and having to make new ones. The conversation went on from there with the participant offering detailed descriptions about being an immigrant and moving from Mexico to Los Angeles.

In a recent study, Rosemann, Palicharla, Rampton, Dyches and Freeborn (2015) used PEI to explore symbols in the lives of children with siblings with autism and Down Syndrome. This research team used photographs taken by the participants to determine differences according to disability, age, and gender of the sibling. The results shed light into the nuanced perspectives of siblings with a disability. By using PEI, the results were not fixed in time or restricted by preconceived constructs. Rosemann et al. found that siblings of children with Down Syndrome took more photographs of people and family members than did siblings of children with autism. Older children took more photographs of the sibling with a disability than did younger children. Sisters photographed people more than brothers. The study's findings facilitated communication with siblings of children with disabilities about meaningful symbols in their lives.

\section{Summary of Methodology Review}

Phenomenological methodology and photo-elicitation interviewing fit well with the aims of this study. Phenomenological research informed nursing practices for parents and infants facing health issues. This research to practice model translated well to the field of AUT-EI. Since the 1950s, PEI has been used to examine individual experiences. Photo-Elicitation Interview offers a means to trigger specifics about memories in a way 
that words alone cannot. Because of its symbolic nature, it also sheds light on deeper personal meaning associated with a phenomena. I selected the Photo-Elicitation Interview for my study to aid my understanding of the participants' lived experiences and special education services. I expected that PEI methodology would trigger meaning of lived experiences that may not be elicited otherwise.

\section{Application of Research Literature to Present Study}

This review identified gaps in the EI-autism literature, particularly the lack of studies examining lived experiences of parents of infants/toddlers with autism. However, the studies on EI, IDEA, Part C, caregiving, and parent perspectives also supported further research into lived experiences in EI-autism. The incongruence between the firstgeneration AUT-EI treatments and IDEA, Part C was an increasing concern among predominant researchers. Caregiving and parent perspective studies indicated that family life was strongly affected by a child's autism. Primary influences included access to services, managing service needs, and quality of interactions with service providers. An understanding of the unique experience caregiving an infant/toddler with autism may offer insight into EI needs and give a voice to parents during a period in their child's development when there is little support or awareness.

My study directly addressed the gaps in the literature and has the potential to inform future intervention efforts for young children with autism in Oregon by describing lived experiences that may be associated with treatment efficacy. This is especially important giving the emergent status of AUT-EI. This study was one of the first to examine the unique lived-experience of parents of young children with autism receiving 
special education services in Oregon. Chapter 3 describes the methodologies and procedures used for this study. Chapter 4 presents the results of the study and Chapter 5 discusses the findings and conclusions. 


\section{CHAPTER 3 \\ METHODS}

"Whoever is searching for the human being must first find the lantern" Nietzsche

Introduction

Chapter 3 presents the methodological procedures undertaken in this study. As an introduction to this chapter I review the theoretical framework and the research questions that guided my inquiry. The emergent nature of phenomenology and engagement between the participants and myself set a context for deep exploration of the phenomena of parenting a young child with autism and the early special education service system. Husserl's (date) descriptive phenomenology provided the methodological structure to bracket my own preconceptions and biases during the research process. Following the review of phenomenology, the remainder of the chapter presents the methods and procedures I employed.

\section{The Phenomenological Research Paradigm}

Overview. Phenomenological methodology is a subset of the qualitative research paradigm. Qualitative research is most appropriate for measuring complex issues such as family dynamics, parent needs, and unique cultural or familial attitudes (Cresswell, 2005). This is in contrast to the role of quantitative methodology in testing a priori hypotheses and seeking empirical evidence to support findings using statistically valid methods. Both methodologies have value in increasing understanding and addressing AUT-EI issues. However, the AUT-EI literature lacked qualitative investigations that may offer insight into parenting a young child with autism and in turn add to existent research and practice. The phenomenological methodology was a suitable approach given 
my goal of gaining insight into the lived experiences of parents with a very young child with autism. Vagle (2014) described phenomenology as a reflective and inductive methodology. Phenomenological research methods involve garnering insight into a person's past, lived experiences as they recollect them. Vagle's distinction between phenomenology and other qualitative approaches helps define this qualitative tradition. Phenomenology explicates the meaning people attribute to everyday experiences. It is in contrast to ethnography (i.e., the study of meaning within specific cultures) and sociology (i.e., the search for meaning in specific social groups). The critical question in phenomenology is "what do we know as persons?" (Reiners, 2012, p. 1).

Descriptive and interpretive phenomenological traditions. Phenomenology is both a philosophy and a research paradigm. As a philosophy, phenomenology can be divided into two major branches, descriptive and interpretive. The German philosopher Edmund Husserl (1859-1938) founded descriptive phenomenology (Reiners, 2012). Husserl's descriptive phenomenological viewpoint was a reaction against positivist views of the late 1800s. Husserl defined individuals as connected meaningfully with everything else in the world (Vagle, 2014). Husserl's descriptive phenomenology aimed to identify the meaning of an individual's lived experiences or to extract meaning from their everyday life. Husserl rejected the assumption that objects existed independently from the subject. However, while a phenomenon is defined by the meaning it has for its subject, anything outside of the immediate experience must be disregarded (Fouche, 1993). To isolate the phenomenon under investigation and interpret the meaning of lived experiences, descriptive phenomenology involves bracketing. Bracketing is the 
suspension of all suppositions to isolate an individual's true lived experience (Vagle, 2014).

Martin Heidegger (1889-1976), a student of Husserl, diverged from the school of descriptive phenomenology and proposed interpretive phenomenology, an ontological theory that addresses the nature of what is being. Heidegger proposed that our understanding of the everyday world is linked to our interpretation of it. Unlike Husserl, who argued that biases and preconceptions must be bracketed to isolate the lived experience of a phenomenon, Heidegger's phenomenology was context bound. As a result, interpretive phenomenology rejects bracketing because the object cannot be separated from the subject's interpretation of it (Groenewald, 2004). Instead, interpretive phenomenology emphasizes co-creation of interpretations between the researcher and participant because all humans share context, culture, and language (Wojnar \& Swanson, 2007).

Phenomenology as a research practice was not fully defined until the 1970s when phenomenological psychologists established a methodology derived from the philosophical tradition (Groenewald, 2004). Phenomenological researchers were concerned with how ordinary people went about their daily lives (Gubrium \& Holstein, 2000) and "understanding the social and psychological phenomena from the perspectives of the people involved" (Welman \& Kruger, 1999, p. 189). Phenomenological data are the participant's descriptions and perspectives related to the phenomenon to be researched (Groenewald, 2004). Gorgi (2009) modified Husserl's descriptive phenomenological philosophy for utility in conducting social science research (Vagle, 
2004). According to Gorgi (2009) the term "description" refers to the narrative data collected from participants about the phenomenon under investigation (p. \#). It also refers to how the researcher analyzes and crafts the narrative data. The participants offer raw data in the form of everyday stories, natural conversation, and reflections about their lived experiences. Descriptive phenomenology involves the researcher reducing the lived-experience information into the phenomenological essences through bracketing, reflection, and data analysis (Vagle, 2004). Swanson-Kauffman and Schonwald (1988) asserted that descriptive phenomenology is the best phenomenological methodology when an inquiry seeks to identify universal qualities of a phenomenon not completely conceptualized in prior research.

Lived experiences and essences. Lived experiences and essences are two constructs in phenomenological research (Vagle, 2014). Lived experiences, as the participant describes them, are used to define the universal structures (i.e., essences) of the phenomenon (De Chesnay, 2014). Lived experiences are the ways in which people live in relation to a phenomenon. Vagle (2014) used the following example to distinguish between other qualitative data and lived experiences. Other qualitative traditions might describe how a tree looks (e.g., the bark, the branches, the leaves). Phenomenologists would be more concerned with how an individual experiences the tree, the personal meaning they associate with it.

The concept of essence found in phenomenological theory and research is described as the universal qualities of a phenomenon under investigation. It is the essential structures of a phenomenon (Vagle, 2014). Essences may be loosely analogous 
to "themes" generated from data in other qualitative methodologies. However, phenomenologists view essences as universal truths that transcend social context rather than trends generated from specific research questions.

\section{Research Questions}

Based on literature review and the two theoretical frameworks for my study (i.e., ecological systems theory and phenomenology), the following research questions guided my inquiry:

1. What are the participants' lived experiences as parents with a young child (under age five) with ASD as they relate to early intervention services?

1a. What are the participants' experiences of having a young child diagnosed with ASD within the past two years?

$1 \mathrm{~b}$. What are the structural essences of the phenomena of parenting a very young child with ASD in the context of EI services?

I selected descriptive phenomenology as the research paradigm for my proposed study because my research questions were concerned with gaining insight into the lived experiences of parents who have very young children with autism. Given a long history of bias and misunderstanding in the field of autism research and practice (Shakespeare, 2013), descriptive phenomenological inquiry was appropriate to make sense of those experiences as they existed without external meaning, bias, or preconceptions. Before introducing the research methodological practices and tools, I present the recruitment strategy and activities undertaken for this study.

\section{Participant Recruitment}

\section{Participant Selection Parameters}

To be selected for this study, participants needed to meet a variety of criteria. Four parents participated in this study. All participants were over the age of 18. Due to 
issues related to ethical concerns regarding children and human subjects requirements, parent participants under age 18 were excluded from the participant recruitment. Early on in the recruitment process selection parameters for child age was problematic. The original study set the age parameter at between 24 and 36 months. However, no parents responded to the recruitment with children in this age range. As a result the child age parameter was expanded up to 60 months. Children of the participants ranged between 40 and 55 months. Consistent with the literature review, parents in this study reported they had difficulty getting an autism evaluation and services prior to age 3 .

All participants were parents of very young children diagnosed with autism. Parents as defined by this study were over age 18 and lived with another primary caregiver and the child with autism. Participants were the primary adults caring for the child and managing day-to-day family life. Parents were also defined as biological or adoptive parents who have been the primary caregivers since birth. There were no racial or ethnic parameters because the focus of this study is the phenomenon of parenting an infant or toddler with autism and the universal essences captured in descriptive data. However, I only included participants whose first language is English. Because I am monolingual in English, a translator would have disrupted the flow of the unstructured interview and compromised the descriptive data collected. A replication study with a multilingual researcher would be more appropriate to address this methodological drawback.

For inclusion in the study, infants were between 24 and 60 months. This age range reflected the current understanding of emergent markers of autism and average age of 
autism diagnosis and commencement of treatment. This criterion was selected to capture the lived experiences of parents with young children with autism who received in EI services. To be included in this study the child with autism had to be the only child in the family with a disability. This restriction narrowed the investigation in order to focus on the phenomenon of first experiences with the EI system with an infant/ toddler with autism. In order for parents to participate in this study, their children must have received EI services through Oregon Education Service Districts (ESDs). Educational Service Districts are Oregon's regional agencies providing of a wide array of educational services to school districts (OAESD, 2016). Early intervention programs through IDEA, Part C are delivered through ESDs. Due to the need to expand the recruitment parameter all children had received EI services, but were currently enrolled in special education services up to age 5 .

\section{Snowball Sampling}

I employed purposeful, snowball sampling (Given, 2008) to select participants. Snowball sampling is frequently used in qualitative research. Qualitative sampling is distinctly different from quantitative sampling methods. While quantitative analysis often involves large, random samples, qualitative investigation involves selection of small, purposefully selected samples. The difference stems from the intent of sampling. Quantitative studies are concerned with generalization and qualitative sampling allows for collecting rich, detailed and thick data (Patton, 1990). As a result the sampling method from this study enabled me to understand and describe rather than control or measure (Miriam \& Simpson, 2000). 
Snowball sampling is an intentional sampling strategy that identifies participants by sampling people who know individuals who generally fit within the parameters set forth in the study. The process begins by asking key, well-situated people who have knowledge about potential participants (Babbie, 1995; Crabtree \& Miller, 1992). Once participants are identified they may refer new participants to the study.

Seven families contacted me and underwent screening for the study. Two participants declined and one did not meet the study's inclusion criteria. I included 4 participants in the study. All participants were mothers of a young child with autism and responsible for primary parenting activities. The small size (i.e., 3-5 participants) was consistent with qualitative research traditions (Cresswell, 2005) and recommended in phenomenological inquiry (Vagle, 2014). By focusing on a small number of participants, I generated extensive narrative data and gained an in-depth and detailed picture of participants' lived experiences. In all the transcribed narrative data totaled over 300 single-spaced pages.

\section{Screening and Participant Selection}

To begin the participant referral process, I sent an email to two ESDs and to five professionals I knew in the field working with families receiving EI services through the ESDs (Appendix A). One of the education program directors I spoke with suggested I contact Autism Society of Oregon as they have online message boards and family support resources. I contacted Autism Society of Oregon and they posted a flyer to their Facebook page. Within two hours I received my first email from an interested participant. This participant proved to be instrumental in my recruitment process. She reposted the 
flyer on her Facebook page and an Autism Mom's Facebook page. Within a week I had seven interested potential participant. The flyer posted on the Facebook pages included the study and my name, telephone number, and email (see Appendices B and C). Recruitment materials outlined the study content, time commitments, and contact information.

I conducted an initial phone screen with the seven individuals who contacted me with interest in participating in my study (Appendix D). The initial phone screen lasted approximately 15 minutes. At the outset of the initial screen, I clarified that my research was not in any way associated with early special education services or ESDs. I also described my role as a phenomenological researcher and a graduate student. Once I explained the study and my role I asked participants if they would like to continue with the initial phone conversation.

In the initial phone screening, I obtained basic demographic information, including verification of child's age (24-60 months), child's enrollment in special education services through Oregon ESDs and an autism eligibility under IDEA. I verified that the potential participant was the parent of the child and responsible for primary caregiving. I also confirmed that the parent was over the age of 18 . I confirmed that there were no other children with disabilities under the parent's' care. Six of the seven participants met the required demographics. I asked the following questions to further screen for study inclusion criteria:

1. Are you currently the parent who is the primary caregiver for the child?

2. Are there any other children in the household who are receiving services for disabilities?

3. Can you provide 10 family photographs of your choice that include the child to 
be discussed during the first interview?

4. Are you available for two 60-90 minute interviews?

5. Are you comfortable with being audiotaped during the interviews?

One participant's answers excluded her from the study; her son was over 60 months. I thanked her for her time and offered a resource list of support agencies for Oregon parents with young children with autism. She declined.

Six parents met the inclusion criteria and expressed interest in participating. I then provided details about the study and its methodological approach, including an overview of photo-elicitation, interview, and phenomenology. Within the methodological approach to the study, I asked participants about their feelings about sharing and discussing family photographs as part of the interview process. Participants were again offered an opportunity to elect out of participation if they felt uncomfortable with the process.

None of the parents had questions or needed information beyond the scope of the study. I offered a resource list either via email or phone (Appendix E) but none requested the list. As part of the initial phone interview, I notified participants that their information would remain confidential and the study was in no way associated with their special education services. A resource sheet was made available to the parents with a list of contacts for family support, information about early intervention and autism (Appendix E), and other agencies supporting families with young children with autism. From the screening of the initial phone interview, I selected four participants and no alternates.

Two of the remaining parents dropped out of the study. One parent participated in the first interview but stated she hadn't collected photographs due to time limitations. We rescheduled the first interview but she did not respond to follow up emails. I thanked her 
for participation. A second participant met the study's criteria during the screening and expressed interest in the study. However, she sent me an email dropping out because she felt that participating in an autism study might jeopardize her privacy and she expressed concerns about the possibility of her child's identity being revealed to family members.

The four remaining participants met the study criteria and agreed to participate I scheduled the first interview sessions with each participant within a week of the screening call. I offered to meet participants in their home or a location convenient to them. I met two participants in their home. I met one participant in a private community room in her apartment building. I met the fourth participant in a private room on campus of a university where she is attending graduate school. I asked each participant to bring at least 10 photographs of their family at the first interview. I asked them to select pictures that included an image of their child but I set no other parameters for photo selection.

\section{Data Collection Methods}

A tenet of Husserlian phenomenology (Author/Date) is that the meaning of a lived experience can only be captured through one-to-one transactions between the researcher and the participant. This involves active listening, interaction, and observation to capture the pure representation of reality in favor of the biased, preconceived representation (Wojnar \& Swanson, 2007). I selected two data collection methods consistent with descriptive phenomenology. I employed photoelicitation, a methodology involving the use of family photographs as part of the interview process. I also conducted unstructured interviews, a preferred method of data collection in phenomenology (Vagle, 2014) because of its utility in gathering rich descriptions from the participants. The following 
sections will discuss these data collections in more detail.

\section{Participant Interview Sessions}

Overview. I conducted two interviews for this study. The first was an unstructured interview guided by family photographs collected by the parent in advance and brought to the first interview. The parents were asked to collect 10 photographs of

their family. The only parameter on photo selection was that the photographs included the child with autism. The participant and other family members or friends may also be in the photograph.

The second interview was a longer, unstructured interview again using prompts to guide the discussion. I prepared for the second interview by reviewing and coding the photoelicitation data and developing individualized prompts to explore the research questions in greater detail. The first part of the second interview was a member check and review of the notes from the first interview. I gave the participants an opportunity to elaborate or clarify our previous conversation. The photographs were available during the second interview and the participants often referred back.

\section{Rationale for using the photoelicitation interview method in this study.}

Photoelicitation has been used in anthropology, special education, and other human sciences (e.g., Byrne, Daykin, \& Coad, 2016; Dennis, Gaulocher, Carpiano, \& Brown, 2009; Latta, 2014). In a review of photoelicitation research, Van Auken, Frisvoll, and Stewart (2010) identified three qualities related to the use of photoelicitation methodology during in-depth interviews: (a) it provides the stimuli for "deep" interviews, (b) it can produce different types of information than other social science techniques, and 
(c) it addresses concerns about power relations between the researcher and subject. All three of these qualities made photoelicitation a good fit for researching the lived experiences of parents of young children with autism. I anticipated that while talking about the pictures they have chosen of their family, parents will offer information about family life and parenting rather than focusing on services and the child's disability alone. While photo-elicitation did elicit deep feelings about family and parenting, service experience played a dominant role in interview content. Talking about photographs offered an opportunity for participants to reflect and make meaning of their world. Photoelicitation interviews often trigger richer conversations about memories, reflections, and experiences than traditional interviewing methods alone (Clark-Ibáñez, 2004; Harper, 2000). Photographs can act as a bridge between cultures (Harper, 2002), making it useful in communicating the family's cultural interpretations of caregiving of a very young child with autism.

In a seminal research study, Collier (1957) found photoelicitation added depth to qualitative interviews and that photoelicitation interviews were longer and more focused than verbal interviews alone. Photographs may trigger responses that would otherwise be missed, enabling the participant to recall specific experiences and sharpen their ability to reflect on and describe their lived experiences and perspectives (Berlin, 1998; Collier, 1957; Harper, 2002; Rose, 2001; Stewart \& Floyd, 2014). Photoelicitation has been described as an anchor between researcher and participant because the in-depth interview is "anchored in the photographic image and understood by both parties." (Harper, 2002, p. 20) that stimulates past experiences in different ways than the verbal interview. This 
allows the researcher access to information that would not have been discussed without the photographic cues (Clark-Ibáñez, 2004). Collier (1957) also noted that photoelicitation mediates the effects of fatigue associated with long verbal interviews. Other fields have used photoelicitation as a way to address concerns related to power relationships often occurring between researcher and participant (Van Auken et al., 2010). Van Auken et al. (2010) pointed out that because participants choose images to be discussed, they retained an element of control over the direction of the interview and topics discussed. As a result, photoelicitation encourages trust between researcher and participant.

Photoelicitation in my study. My study is one of the first to explore parents' lived experiences of parenting their young children with autism and receiving special education services. It is one of the first to employ photoelicitation interviewing. Using photographs offered an alternative way for parents to describe the personal meaning attached to their experiences. The results of the study offer an alternative to preconceptions associated with autism's history of stigmatization in past research and practice (Glynne-Owen, 2010; Shakespeare, 2013).

Photographs as data. In addition to aiding participants in recalling and relating family experiences, photoelicitation allowed for another perspective on the lived experience. The photographs themselves were analyzed for content (Mandleco, 2013). The selected photographs offered insight into family culture and priorities through analysis of photographic content (e.g., places, individuals, events). In addition, the photographs were linked to the interview transcripts and analyzed to identify why the 
participants chose each photograph was and the meaning they attributed to the image.

Unstructured interviewing in phenomenology. Unstructured interviews are the most popular interview method in phenomenological research (Vagle, 2014).

Unstructured interviews are dialogic, conversational, and open. Like phenomenological theory, unstructured interviewing is in contrast to quantitative design, which relies on distance and control. A fundamental goal of in-depth interviewing is understanding lived experiences. Unstructured interviews were well suited to my proposed study given the generative nature of unstructured interviewing and my study's goal of understanding as much as possible about the lived experiences caregiving for an infant/toddler with autism. By engaging in open conversations with the participants, I was able to gather information about how they make meaning of their lived experiences (Seidman, 2013).

Larossa and Wolf (1980) described the unstructured interview in the participant's home as a context for discussions with the participant that generates information that might not have otherwise been revealed. Unstructured interviews aid in collecting rich information and provide an opportunity for clarifications and addressing misunderstandings. Given the goal of examining the participants' meaning of their own lives, unstructured interviews were a good fit for my study. Unstructured interview were selected over semistructured methods because of the unique characteristics of unstructured interviews. Corbin and Morse (2013) compared the two interviewing methods

[During the unstructured interview] the participant has the control over the pacing of the interview, what will be disclosed (the amount of detail, scope of the interview, etc.), and the emotional intensity. [During the semistructured interview] the participant may withhold important information because the 
relevant question was not asked, may answer in a perfunctory manner, or not fully cooperate. (Corbin \& Morse, p. 340)

Despite the conversational, dialogic nature of unstructured interviewing, the interviews themselves were not without focus and intention. Vagle (2014) addresses the myth that unstructured interviews are wide open and without boundaries. Rather, the interview needs to be disciplined by the research questions themselves and a constant orientation toward the phenomenon under investigation. In order to maintain focus on the phenomena I used a set of prompts that structured the dialogue between the participant and myself. I used the same prompts with all participants (Appendix F) While specific questions structured the interview, unlike semi-structured questions, the participant selected from the set of questions to begin the dialogue (Anderson, 2015). There was no set order to the questions and participants were asked to elaborate on their ideas and meaning. While the data collection methods were unstructured, the prompting strategies kept the focus of the conversation on the phenomenon and supported participants in exploring lived experiences in a dialogic, open, and conversational manner consistent with the phenomenological paradigm (Vagle, 2014).

An important distinction between phenomenological research methodology and empirical study of the human experience is the dialogical data collection process. In the case of unstructured interviewing the participant takes a significant role in determining what is said. During the conversations I engaged the participants through questions like "tell me more about that," or "I have an understanding of that phrase you used, but can you tell me what it means to you?" (Vagle, 2014, p. 81). This encouraged the participants to elaborate on topics generated through the prompting system described above. 
Limitations of interview methodology. There were limitations to using interviews as a data collection method. The interviews were time intensive and required extensive participant involvement. Each interview lasted between 1 and 1.5 hours and one participant and I met on three occasions, our conversations totaling more than 5 hours. There was a risk that as participants discussed their personal narratives about their family; they may have felt vulnerable or exposed. I asked participants throughout the interview process if they needed a break and informed them that they did not have to answer any questions they didn't want to. Further during the second interview I asked them about their experience with the process of photo-elicitation interviews. All participants reported it as a positive experience. None of the participants in this study reported feeling exposed but it remained a limitation of the unstructured photo-elicitation methodology. A third limitation of the interview methodology is self-reported information is subject to biases both on the part of the participant and the researcher. Merriam (2009) points out that the relationship between the interviewer and participant is a complicated one. Biases, predispositions, attitudes, and preconceptions can affect the data's validity. Descriptive phenomenology offered a means to address these complexities through bracketing methods to identify researcher biases and preconceptions (Vagle, 2014). I used strategies to enhance the participant's trust and comfort. I was respectful, and engaged in active listening (Yin, 2014). All of the participants reported that they enjoyed the interview process and in addition to contributing to a cause they felt worthy they felt the process was positive on a personal level. 
Audio recording the interviews. Each interview was audio recorded. The recording started after I reviewed the informed consent at the beginning of each interview. The audio recording concluded at the end of the participants answer to the final prompt. All recordings were time and date stamped. The audio was transcribed verbatim. Only I, as researcher had access to the transcriptions and they were coded with participant pseudonyms and date.

\section{First Interview Procedures}

Interview structure, script, and interview prompts. The first meeting with the participant was an unstructured interview guided by photographs. During the first interview I became acquainted with the participant, family life, values, and culture. A copy of the first interview script can be found in Appendix F.

I set up the interview according to the following design. I met the participants in locations convenient to them. Two participants invited me to their home. The other two preferred a quiet meeting place other than their home. Once I reviewed the informed consent and offered an overview of the meeting structure, I asked the participants to spread out the photographs however they liked. I placed the printed prompts randomly on the table. One parent shared photographs from her cell phone. I photographed and noted the order of the pictures for data analysis. We used the same prompts as with the other three participants.

Once we settled in I provided an introduction to the study and I reviewed the informed consent document (Appendix G). Each participant received a copy of the informed consent form before we started the first interview. I provided another copy of 
the informed consent at the second interview. On each occasion I reminded the participant that we can stop at any time. I reminded them that they can drop out of the study at any time for any reason. I also let the participant know that if there have questions outside of the scope of the study, I will offer resources. I gave each participant a copy of the resource sheet.

I explained that photographs were to be used as a way for them to share their parenting experiences and that this method might help me understand their lived experiences in a deeper way than with talk interview alone. I explained that photoelicitation interviewing is a way to tell the story of their child's life and their experiences as a parent. I intentionally informed them that there are no correct or incorrect responses and that I was interested in the unique way they tell met about their experiences.

I placed four prompts on the table to help to focus the participants. These prompts were:

1. Pick a photograph and tell me what's happening in the picture?

2. Select one of the photographs and tell me why did you select it to talk about during our interview?

3. Select a photograph and tell me about the people in it and their relationship with your child?

4. Select a photograph of an event that you remember well, tell me about that day and what your family was doing?

Once participants had a chance to read over the prompt questions for interview 1 , I asked them to select a photograph and choose a prompt to begin our conversation. As part of the process, I often asked parents to elaborate on an idea or to confirm my understanding of their statements.

Prior to ending the first interview I scanned the photographs. Three of the 
participants offered to let me keep the original copies of the photographs. I accepted as I felt it would aid in my analysis of the photographic data. These images were stored with the other research materials in a locked file cabinet in my home office. At the end of the interview I thanked the participant for meeting with me. I also informed them that they are welcome to call or email me in between sessions if they think of anything they wanted to add or correct. Before leaving I scheduled our second interview for approximately two weeks later. All artifacts from the first interview were coded and recorded with an artifact catalogue and stored in a locked file cabinet in my home office (see Appendix I).

As part of the research process, I reflected on the participants' experiences during the first interviews and on the information they shared with me. I used a research journal and included my thoughts on the study activities. I also used the journal for bracketing activities that explored my assumptions and potential biases. I reflected on biases that may have influenced some of the participants' responses. I engaged in reflective activities consistent with phenomenological research methods. This included bracketing and journaling about the content of the interviews and the feelings I had about the process. It also included debriefing and conversations with a phenomenological expert. Additionally, I found I began to have a phenomenological mindset, engaging in self reflection about the process outside of the formal methodological practices. These reflective activities are described later in this chapter.

I transcribed the audio recordings, analyzed the photographs, and continuously engaged in reflective activities to help me understand participants' experiences and 
explore each experience's essence. As the essential themes emerged from the data I began exploring essential themes within the context of several metaphors. Originally, I envisioned the model for the phenomena as "parents as super heros" and their experiences within a good vs. evil narrative. I spent quite a while exploring this metaphor. However, after the second interviews were completed I found that the parenting experiences was not a static world where the parent is always "fighting for good." Yes, these parents remained "super" to me. They demonstrated resiliency and fortitude beyond that of parents of neurotypical children. And while their world was indeed one made for neurotypical children, the parents were also on a journey. They were forging their way as pioneers, blazing a trail for future parents of very young children with autism. When my advisor suggested the Oregon Trail I could see the metaphor fit, even in its reference to Oregon because each state has its own early special education systems and these parents were indeed navigating with their families to a better place.

\section{Second Interview Procedures}

Interview structure, script, and interview prompts. The second interview involved deeper discussion about the parent's lived experience as the primary caregiver for their young child with autism. I used the first part of the second interview as a member check, reviewing my notes from the previous interview. I did this as a bracketing strategy to make sure I captured the parent's personal meaning. I also used it to give the parents an opportunity to reflect on their own responses. I used notes from the first interview and created individualized prompts to more deeply explore parents' lived experiences with EI services. A copy of the second interview script can be found in 
Appendix H.

During the second interview, I met the participants in locations convenient to them. I met with three participants in their home and one participant in a meeting room in her apartment building. We spread out prompts printed on white sheets of paper. We also had the photographs from the first interview available and referenced them. I started the audio recording prior to the beginning of our interview. The recording ended after the participant finishes answering the last question.

Once we settled in, I outlined the structure of the interview and reiterated that we can stop at any time in the process. I structured the second interview as follows:

1. I restated the objectives of the study, the informed consent procedures. I reminded the participant that we could stop at any time. I gave the participants a second copy of the informed consent form. I let the participant know that if there were questions they have outside of the scope of the study, I would provide a resource sheet. I offered them another copy of the resource sheet but none of the participants requested a second copy.

2. I reviewed the themes from the first interview and asked participants if I captured their story accurately. I gave the participants an opportunity to elaborate or change answers. They often elaborated during this member checking procedure.

3. I then placed several prompts on the table to help to focus the participants. The prompts were individualized for each participant based on the data collected and analyzed from interview 1 (see Chapter 4 for additional information on individualized prompts). The prompts focused on following three themes:

a. Elaborations on family events or experiences (e.g., At our first interview we discussed can you tell me more about that experience?).

b. How EI services impacted your family life. (e.g., The last time we spoke you told me a story about when your child was diagnosed with autism can you tell me more about that event?)

c. What it means to have a child with autism. (e.g., You have mentioned several times that parenting a child with autism is a journey. Can you tell me more about that?) 
Once participants had a chance to read over the prompt questions for interview 2 , I asked them to choose a prompt to begin our conversation. As part of the process, I asked parents for more information or if there is anything else they would like to add.

Prior to ending the second interview I thanked participants for their participation. Before leaving I asked them if it would be all right if I contacted them for more information. I also let them know I would email them their transcripts within a month so they can review to confirm I understood their responses and perspectives. All artifacts from the first second were coded and recorded in a an artifact catalogue and stored in a locked file cabinet in my home office (Appendix I).

As part of the research process, I reflected on the participants' experience shared with me during the second interview. I engaged in phenomenological activities alone and in partnership with an expert in phenomenology. I transcribed the audio recordings, and combined first and second interview data. I engaged in creative and reflective activities to help me understand participants' lived experiences. This involved looping and revisiting the data. It was a dialogic process whereby the lived experiences untangled themselves from the larger body of data. I identified essential themes across participants. A part of this process was to engage in activities to bracket out my preconceptions and biases and also identify possible biases and preconceptions by the participant. These reflective activities are described later in this chapter.

Additional data collection. I added two data collection activities so I could gain a better understanding of participants' lived experience. These activities emerged as a result of engagement with the first round of data collection and analysis. I added (a) a 
third interview with one of the participants (i.e., participant 005, "Karen") and (b) a universal member check meeting.

Third meeting with Karen. The fourth participant "Karen" and I talked for over 3 hours during our first two interviews. We had only discussed 5 of the 10 photographs and we both felt a third interview was needed in order for me to understand her lived experiences as a mother of a very young child with autism receiving early special education services. The third interview proved valuable. The last five photographs and conversations they generated contrasted with the first five. Karen had selected this group of pictures to share the change in her parenting experiences from hardship to joy.

Universal member check group meeting. By the second interview sessions, I had already begun analyzing the interview data. As I did I realized how much value a universal member check would add to the validity of the essential themes. I asked the participants individually if they would be interested in meeting with the other four participants and reviewing my results from the study. All participants were eager to do so. I amended the study's Human Subjects application, submitted it to Portland State's Institutional Review Board, and obtained approval prior to the universal member check meeting. All four participants attended a group meeting at private room in a pizza place in a central location close to their homes.

\section{Maintaining Data}

I scanned the completed informed consent forms and uploaded them to a password-protected computer. I destroyed the original forms. I scanned the family photographs following the first interview and uploaded the images on a password- 
protected computer. The participants offered to let me take the photographs for analysis. These were stored in a locked cabinet in my home. I did not include photographic images in the dissertation. Instead I replaced them computer generated sketches with unidentifiable faces. To protect their identities and insure privacy, I used pseudonyms for each participant in the final dissertation. I used a numeric coding system during the data analysis process. I never used their names, names of their children, or names of other individuals, or places they mention in the course of our conversations unless I was communicating with them directly.

I stored the transcribed audio data is in a password-protected file on my computer (along with the interview notes and digital family photographs). I scanned all hard copies connected with the project and stored them in a password protected file on my computer.

Digital uploads will be destroyed within six months after the data analysis is completed. All notes will be destroyed after the dissertation defense or within five years, whichever comes first. All consent forms will also be kept for five years.

\section{Role of the Researcher}

As a researcher for this study, I was committed to the highest ethical standards (Yin, 2014). These standards include honesty, responsibility, competence, and credibility. I was also committed to respect and empathy toward the participants with whom I worked. With these aims in mind, I also acknowledge that qualitative research is fundamentally a human endeavor and as such I was subject to bias. I do not have a child with a disability, but have studied and worked with families of children with autism for over 10 years and in special education for 25 years. I am also committed to advocacy and 
strengths-based EI philosophies, which is very much aligned with phenomenological investigation. I have conducted two research studies examining parent perspectives in families with young children with autism. These studies provided a background from which I developed the research questions for this study. I have worked directly with children with autism and their families. I have also coached early childhood education teams working with young children with autism. Further, I held research positions on both qualitative and quantitative studies related to parent/infant interaction, early autism, and social-communication and play in autism. I am aware of the quantitative research and treatment precedents in AUT-EI and the need for a greater understanding of the lived experiences of parents of young children with autism. I hold a Master's degree and Oregon Teaching license in Early Intervention/Early Childhood Special Education. I currently teach early childhood development, early intervention, and infant mental health courses at Portland State University. I have experience advocating for children and families with disabilities having worked as an educational consultant, family advocate, and expert witness. My background is well suited to conducting this study. I have expert knowledge on this area of research and am strongly committed to supporting and advocating for families. Phenomenological research is a good fit for me because of my desire to give voice to parents of young children with autism.

In my work in special education and mental health as a consultant, advocate and instructor, I have observed issues related to problems from research to practice. These problems of practice inform my research. I do not assume that programs are implemented with fidelity or teachers or providers have received training in person-centered planning, 
collaborative practices, and strengths-based assessment. I hold the opinion that a flaw in $\mathrm{ABA}$ and other structured, specialized strategies is a vulnerability to inflexibility in implementation. My observations and opinions are consistent with other experts in the field. I have observed problems with fidelity of implementation in educational teaching practices. Applying ABA in home or school settings requires a nuanced, contingencydriven, and flexible approach to teaching. I have observed inexperienced or untrained teaching staff applying specialized practices in such a way that is aversive to the children because it is being delivered as a set of prescribed procedures despite the experience for the child. I've frequently witnessed children 'crying through' intervention activities, held down during assessments, and ignoring bids for communication. I've also supported families who have felt marginalized by interventionists and educational programs without the resources or skills to practice collaboration and strategies to support family capacity and self-efficacy. As a college instructor, I have recently taught students in the last year of their coursework who were not familiar with collaborative practices required by the Individuals with Disabilities Education Act.

These experiences informed my work as a qualitative researcher. In selecting phenomenology, I was committed to a methodological paradigm that isolates a phenomena and its meaning from the researcher and participants' preconceptions. This was a reflective process and I engaged in activities to interact with the data collection and analysis through many lenses. I maintained a personal commitment to remain open to findings that challenged to my personal beliefs. I feel I was flexible and reflected on my beliefs, opinions, and expectations as I gathered new information. 


\section{Data Analysis}

I used two methods of data collection (photoeliecitation and unstructured interviews). I synthesized these two sets of data to determine the lived experiences and essences of the phenomena of caregiving for an infant or toddler with autism. I used a method described by Mandleco (2013) to analyze the photographic data and Colaizzi's (1978) method for bracketing and analyzing the interview narrative data. These two data analysis methodologies are discussed in the following sections.

\section{Mandleco's Method for Photographic Data Analysis}

Mandleco (2013) described a system for analyzing photoelicitation data. This method involves (1) numbering each photograph, (2) using open coding to sort the photographs according to image composition, (3) using interview transcripts to assign participants' meaning associated with each photograph (e.g., we love family activities or her brother always makes her laugh), (4) using the transcript to determine the identity of the individuals and location of the photograph, (5) determining and verifying categories using qualitative methods (Denizn \& Lincoln, 2000), and (6) analyzing the importance of the photographs to the participant.

\section{Analyzing Unstructured Interview Data}

De Chesnay (2014) outlines four steps in the analysis of data in descriptive phenomenological research: (a) bracketing, (b) analyzing, (c) intuiting, and (d) describing. This section will describe each step in this process.

Bracketing. I engaged in specific activities that served to neutralize my personal biases and preconceptions. I used bracketing strategies throughout the phenomenological 
research process. Bracketing activities were reflective and included keeping a diary about my perceptions and feelings, recording field notes, and seeking critiques from a methodological expert in descriptive phenomenology (Vagle, 2014). In addition I read and re-read interview transcripts and reflected on the meaning of the participants' lived experiences and reflected on possible biases held by the participants' and myself. Additionally, as the rapport between the participants and myself evolved, I shared my preconceptions as they came up naturally in our conversations. I gave the participants an opportunity to comment. Three participants sent emails after the interviews. These emails helped them clarify their perspectives and elaborate on experiences.

Analyzing. I employed Colaizzi’s method for data analysis (as cited in De Chesnay, 2014). Colaizzi's method is commonly used in phenomenological research analysis and involves seven steps (pp. 147-148).

1. Reading and rereading the participants' descriptions of the phenomenon to acquire a feeling for their experience and to make general sense of their experience.

2. Extracting significant statements that pertain directly to the phenomenon under study.

3. Formulating meanings for these significant statements to illuminate hidden meanings.

4. Categorizing the meanings into clusters of themes and confirming consistency between the emerging findings and the participants' stories without giving in to the temptation to ignore data that do not "fit."

5. Integrating the findings into an exhaustive description of the phenomenon under study; describing includes coding segments of text for topics, comparing topics for consistent themes, and bridging themes for their conceptual meanings, which leads to creating a prototype of a theoretical model about the phenomenon studied.

6. Validating the findings by returning to the study participants to ask how the universal description compares with their personal experiences.

7. Incorporating any changes offered by the participants into the final description of the phenomenon. 
Intuiting. Intuiting is a reflective activity whereby I tried to understand the experience from the participants' point of view and begin to consider the commonalities in the participants' descriptions of their lived experiences. This was achieved through active listening and reflective practices (e.g., reflection following a conversation with the participant, recording thoughts in a diary, conferring with another phenomenological researcher). I engaged in intuiting by withholding final interpretation yet allowing opinions to emerge about the universal essences in the participants' narratives.

Describing. The final stage of data analysis in descriptive phenomenological research is presenting a model of the structure of the phenomena under investigation. If the true essences are captured in the model, then anyone who has experienced the phenomenon should be able to recognize the features of their lived experience in the model or description.

\section{Addressing Research Validity}

I modified Lincoln and Guba's (1989) model to address validity in my study. I selected data analysis methods consistent with the researcher activities described by Lincoln and Guba (1989). I focused on three areas to establish validity in my study: (a) credibility, (b) transferability, and (c) dependability.

Credibility. Credibility is confidence in the "truth" of the findings. In phenomenological research, the "truth" is an accurate model or description of the universal structure of participants' lived experiences. Lincoln and Guba (1989) described researcher activities that promote credibility in qualitative research. I used several of the research activities consistent with Guba and Lincoln's standard: (a) persistent 
observation, (b) triangulation of data, and (c) peer debriefing.

Persistent observation. I use unstructured interviews and maintained a flexible researcher stance. These strategies allowed me to gather rich and detailed information about the participants lived experiences parenting a young child with autism. I retained an open stance and utilized additional data collection methods to fully explore the phenomena under investigation. I conducted reflection and data analysis within and across participant data (transcripts and photographic data). I was able to identify relevant data and bracket out irrelevant (non-essential) observations (Onwuegbuzie \& Leech, 2007). An example of looping back and excluding irrelevant themes is when I analyzed the photographic for chronological patterns. While participant data as a whole revealed a relevant chronological trend about participants' parenting experiences, not all of the participants selected or presented photographs chronologically. I analyzed the participants' photographic presentations and reviewed the transcripts to confirm this trend across participants despite the ways the participants chose to share their photographs.

Triangulation. Triangulation involves a combination of methodologies to study the same research problem (Onwuegbuzie \& Leech, 2007). I employed triangulation in my study design in order represent the phenomenon as accurately as possible by obtaining corroborating evidence. I employed two types of triangulation in my study (Denzin, 1973): (a) data triangulation (i.e., photographic, interview, group interview) and (b) theory triangulation (i.e., phenomenology and Ecological Systems Theory).

Peer debriefing. I worked closely with a phenomenological researcher to discuss 
my interpretation of the data and participants' experiences. These deep conversations included bracketing and debriefings and helped me identify and bracket my own biases and preconceptions about participant experiences. These meetings also served to explore lived experiences extracted from the data and the universal essence or phenomenological model.

Transferability. Transferability is showing that the findings are applicable to other contexts. This concept is similar to generalizability in quantitative research. In phenomenological research, the goal is to arrive at a description of the universal structure of a phenomenon. Because it is a unique phenomenon under investigation, it is not expected that the findings will transfer to other phenomena. However, if the phenomenological researcher has been successful, any individual who shares that lived experience should be able to recognize the model or description (De Chesnay, 2014). However, because phenomenology is less concerned with objective statements about theories or trends, transferability beyond the phenomena under investigation is not a goal. I addressed transferability through a universal member check procedure whereby the participants confirmed the essential themes (i.e., the strands of the universal structure) from the data analysis.

Dependability. Dependability refers to rigorous and organized research practices. The methodological practices I selected for this study involved clearly stated and organized research procedures. An independent auditor conducted a dependability audit of my research practices at every phase of the process. The auditor reviewed my research activities and confirmed consistency between proposed methods and research activities. 


\section{Chapter 3 Summary}

I conducted a phenomenological investigation of the lived experiences of parents of very young children with autism. The study employed photoelicitation methods and unstructured interviews to gather fine-grained data about the participants' lived experiences. I used two rigorous methods for data analysis: Mandleco's (2013) method for analyzing photographic data and Colaizzi’s (1978) data analysis method for descriptive phenomenological research. Throughout the research process I (a) engaged in research activities that fostered trust between the participant and me, (b) employed bracketing activities to neutralize biases and preconceptions, and (c) implemented research strategies consistent with Guba and Lincoln's (1989) standards for qualitative research validity. 


\section{CHAPTER 4 \\ RESEARCH FINDINGS AND DISCUSSION}

\section{Introduction}

Chapter 4 explains how participants expressed their experiences parenting their young child with autism and navigating early intervention services. The literature review in Chapter 2 revealed the complexity of parenting a young child with autism and the influences of nested ecosystems as defined by ecological theories of development (Bronfenbrenner, 1978). Using Husserl's (1970) descriptive phenomenology the exploration of each participant's individual lived experience led to an examination of essential themes related to parenting a young child with autism and interacting with the myriad of early autism services.

Phenomenology differs from other qualitative research traditions. Rather than focusing on themes emerging from empirical data analysis, descriptive phenomenology applies reflection and rigorous analysis of lived experiences in order to identify a universal structure of the phenomenon under investigation (Vagle, 2014). Phenomenological themes are not generalizations. Rather, they are strands that form the larger meaning of the phenomenon. Through researcher bracketing activities and engagement with the data, researcher and participants' biases and preconceptions are bracketed out (Vagle, 2014). Thus essential themes reported by all participants describe the phenomenon in a manner that would conceivably be recognizable by anyone experiencing the same phenomenon (Vagle, 2014; (Wojnar, Swanson, \& Schonwald, 1988).

I used photo-elicitation interviews (Collier, 1957) as a way to support 
participants' memories and descriptions of lived experiences. Each of the four stories in this chapter represents a unique lived experience shaped by participants' culture, family composition, worldview, and support resources. As the participants' stories took shape during the research and analyses, essential themes became apparent. These essential themes echoed findings from the literature review, specifically the prominence of service navigation in everyday lives of parents of very young children with autism (Osborne \& Reed, 2008). These will be discussed in more detail in chapter 5 .

The use of photographs as part of the interview process allowed the participants' to reflect on emotional, as well as, practical aspects of parenting a young child with autism and navigating service systems. The photographs "anchored" (Loeffler, 2005, p. $345)$ the participants' experiences in milestone events for their child and their journey to autism treatment.

Throughout the interview and research process my experiences were woven with the participants'. I employed bracketing strategies (Vagle, 2014) to address my past experiences with autism research and my preconceptions about early intervention services for children with autism. Bracketing is a concept in descriptive phenomenology whereby the researcher engages in reflective activities to identify and "bracket out" preconceptions and biases (Vagle, 2014). Through phenomenological research methods I grew to know the parents on a personal level; I came to understand their lived experiences and meaning beyond both of our preconceptions about parenting and early intervention service models.

Chapter 4 is organized in two sections. Section One is a presentation of individual 
participants' narratives. This includes the lived experiences and personal meaning participants attributed to the phenomena. Section one addresses the study's research questions and reflects my bracketing practices that isolated the true meaning of the participants' lived experiences. The participants shared their lived experience in prompted but unstructured interviews aided by participant-selected family photographs. Each participant collected at least 10 photographs. I paid careful attention to the order of the photographs, the participants' descriptions, and emotional reactions to events depicted therein. Participants presented, in their own words, examples of parenting a young child with autism and navigating early intervention services. The participants reported that the photographs aided in provoking and capturing their unique family experiences. Throughout the narratives, I provide my personal-researcher reflections drawn from my bracketing journal and reflective methodologies. I recorded my bracketing activities in my journal but they represented not merely reflections but also evidence of the manner in which I set aside suppositions or held in "abeyance assumptions surrounding the phenomenon" (Gearing, 2004, p. 1433). Descriptive phenomenology requires isolating the phenomenon through identifying and bracketing out bias and preconceptions on the researcher, participant, and socially constructed levels (Vagle, 2014). The goal of descriptive phenomenology is to describe the phenomenon without "interference of interpretative influences“" (Gearing, 2004, p. 1436).

The four narratives represent the interviews, bracketing and reflection, listening to audio recordings of the interviews, and repeated transcript review. In addition, I engaged participants in reviewing transcripts and interview summaries as a safeguard against 
misrepresentation of their experiences and to correct biases I missed during bracketing activities.

In Section Two I present the essential themes that illumined themselves during the research and data analysis. These essential themes formed the structural model of the phenomena. The concept of essence or essential themes found in phenomenological research is described as the universal qualities of a phenomenon under investigation. It is the essential structure of a phenomenon. While Husserl (1970) viewed essences as philosophical universal truths that transcend social context, contemporary phenomenologists view essential themes as an accurate description of the meaning of a human experience without interference from outside interpretations (Gearing, 2004; Vagle, 2014).

I engaged in intuiting by withholding final interpretation yet allowing opinions to emerge about the universal essences in the participants' narratives. I patiently waited and reflected as I engaged in looping and revisiting the data. It was a dialogic process whereby the lived experiences untangled themselves from the larger body of data. I often wrote in my reflective bracketing journal "the shared lived experiences seemed to float to the top of the narratives." As a researcher I maintained continuous engagement and reflection with the data by listening to the audio recordings, reading, and summarizing the transcripts, and member checking essential elements of the phenomena. A final universal member check confirmed validity of the themes as I interpreted them. The participants and I met as a group and they confirmed the universality of the essential themes. I present the essential themes in section two as strands in a universal model of 
the phenomena. After identifying essential themes through a series of reflective and creative research activities, the participants' lived experiences resulted in a woven narrative of shared experiences. The parents' journeys consisted of discrete phases, temporally and experientially. The unchartered territory of raising a very young child with emergent autism and navigating the early intervention systems led parents into a new role and they blazed a trail for the next generation of parents. The trail from embarking on the unknown to establishing EI services emerged as the phenomenological paradigm: (1) Parents as Pioneers, (2) Making the Journey as a Family, (3) Navigating the Undeveloped (New World) of Autism Early Intervention Services, (4) Challenges and Obstacles, (5) Ingenuity, Resilience and Perseverance, and (6) Reflecting on the first three years and looking forward.

\section{Review of Methods}

The four mothers participating in this study contacted me via email after seeing a flyer for my study posted on social media. Initially, the flyer was posted on a local chapter of Autism Society. However, the flyer had been reposted to several Facebook groups and other social media sites frequented by parents of children with autism. All four mothers participated in an initial phone screening followed by two interviews. During the phone screening I asked the participants questions to confirm they met the study's criteria and requirements.

All four mothers participated in a photo-elicitation interview and follow up interviews. Additionally, I added a universal member check group meeting to the original study design to strengthen the validity of my conclusions essential themes and 
the phenomenological structure. I engaged in bracketing activities throughout the process. This included journaling immediately following each interview. I used reflection to analyze topics, participant responses, and to isolate preconceptions I brought to the process. I immersed myself in the audio and photographic data. I compared the parents' responses with my own personal experiences parenting a young child. I drew comparisons to the literature and returned frequently to the research questions.

\section{Research Questions}

1. What are the participants' lived experiences as parents with a young child (under age five) with ASD as they relate to early intervention services?

1a. What are the participants' experiences of having a young child diagnosed with ASD within the past two years?

$1 \mathrm{~b}$. What are the structural essences of the phenomena of parenting a very young child with ASD in the context of EI services?

\section{The First Interview}

I structured the first interview using a set of 10 family photographs the participants collected in preparation for our interview. One function of collecting photographs as preparation for the interview was to orient the participants to the study's investigation. Photographic interviewing can serve as memory anchors (Loeffler, 2005, p. 345) as participants recall the moment in the photograph. The photograph aids in accurate recall of past events and allowing participants to access memories in a way that cannot be captured through verbal responses alone (Collier, 1957). Thus, family photographs yielded rich and detailed qualitative descriptions. Participants used the photographs as references for their lived experiences. I used a series of written prompts to guide the interview process. Participants placed the photographs on the table in any order. I 
randomly placed the written prompts around the table. The four prompts were:

1. Pick a photograph and tell me what's happening in the picture?

2. Select one of the photographs and tell me why did you select it to talk about during our interview?

3. Select a photograph and tell me about the people in it and their relationship with your child?

4. Select a photograph of an event that you remember well, tell me about that day and what your family was doing?

\section{The Subsequent Interviews}

Following the first interview, all parents participated in subsequent interviews. Three participants met with me for a total of two interviews while the fourth participant and I met on three occasions. The second interview functioned as a member check of experiences as I interpreted them from the first interview data. The participants and I also continued our conversations about their lived experiences aided by an individualized set of prompts. We referenced to the photographs during all interviews.

\section{The Universal Member Check Meeting}

Once the data collection and first round of member checking was completed, I realized that a universal member check meeting would add validity to my essential themes. I asked each participant individually if they would be interested in meeting as a group to talk about the study's results. I told them there was no obligation to do so. Once they all expressed an interest I added an addendum to the Human Subjects Application. Upon approval, I scheduled a meeting with the four participants in a location convenient to everyone. We met in a private room at a pizza place. I shared the universal themes and talked with them to insure I captured their experiences and to confirm the themes as universal across all participants. 


\section{Section One: The Four Participant Narratives}

\section{Introduction to Section One}

In section one I present the four participant narratives about parenting their young child with autism and early special education services. I used pseudonyms for the participants and other family members. Many of the participants' photographs are presented with the narratives but at times only descriptions of photographs are used at a participant's request. All photographic images were digitally altered to obscure participants' identities. The benefits of including recognizable photographic images in the findings did not outweigh the risk of breaching participant privacy.

The narratives in Section 1 address the study's first research sub-question: (1a) What are parents' experiences of having an infant or toddler recently diagnosed with ASD?

Each narrative is supported by the participants' own words, directly quoted from the audio transcript. I synthesized data from both interviews presented it as continuous representation of the lived experiences from which they were drawn. Indentation and single spacing is used to delineate the participants' verbatim responses. Throughout the narratives I included notes about my bracketing activities to illustrate my engagement with the data as a phenomenological researcher. Each participant narrative is organized into subsections: meeting the participant; family life; early autism symptoms and diagnosis; raising a child with autism in a neurotypical world; challenges and obstacles; and interview two: a deeper look at service experiences. 


\section{Narrative 1: Caroline's Lived Experiences}

Meeting Caroline. Caroline and I met in a meeting room at the university where she was finishing her doctoral degree in social sciences. Caroline was excited to participate in the study. She had been instrumental in my successful recruitment of participants. She said she had seen my flyer on the local Autism Society website and immediately reposted it and forwarded it by email to potential participants whom she thought met the inclusion criteria. Caroline expressed that she thought my study was important and wanted to help parents' voices get into the research. Caroline shared that she was finishing a doctorate in social sciences. I found out during the course of our interviews that she also held two master's degrees. Caroline is married and has two children, Joshua (4) and his younger brother (2).

Caroline brought ten photographs for the interview. I asked her to lay them out on the table and then I placed the prompts around the table. She told me that she preferred to talk about the photographs chronologically. Caroline lined the pictures up in a row on the table in front of us from the earliest to the most recent. She shared with me that she herself is autistic and tends to prefer order and routine. Our interview lasted about an hour. We talked with the aid of the written prompts and the photographs. Often Caroline picked up a photograph while she shared a story with me.

Family life. Caroline looked over the prompts and picked "select a photograph that you remember well and tell me about that day and what your family was doing." She picked up photograph C-1 (photo redacted) ${ }^{2}$ The photograph depicts Caroline's husband

\footnotetext{
2 Descriptions of photographs are used in cases where Caroline did not want the images used in the
} 
sitting at the desk in front of his computer. Nathan, about three-years old, has his arms around his father's neck. His feet are over the back of the chair and his body forms a bridge between the back of the chair and his father's shoulders. She told me about the picture, punctuating the story with laughter and affection. It was Christmas last year and her husband was Skyping with out of town relatives. Her son with autism, Nathan, was on the back of the chair with his arms around his dad's neck. Caroline smiled as she looked at the picture and told me about the scene and said it represented their family life so well. I got a strong sense of parental pride as she talked to me about Nathan. She told me that Nathan was so excited because of Christmas and that "he desperately needed input. It was one of those days." It was cold outside and they had been cooped up all day. This photograph represented their life and how Nathan is "doing his thing" and getting the sensory input he needs. She laughed and told me she could only imagine what they were thinking on the other end. Maybe something like "Gosh, he's hyper."

CAROLINE: Well it's so typical ...like so many things in our life. [for example] him bouncing off of the walls or just some of the accommodations that we have to do are so part of the bill for us....So that's why I chose this picture ...it shows him filling his needs in a very unusual and silly way and my husband is just kind of like it's life...

I then asked Caroline how the family members on the other end of the Skype call responded to Nathan climbing on his dad. Caroline paused for a moment before answering my question.

CAROLINE: It's... heartbreaking because his family wants to connect with him. We don't see family very often. They really want to connect with him. They want to talk to him how they would talk to typical kids and he just can't do that. We...try and provide coaching. Or we will try and provide an explanation...we

published dissertation. 
also kind of have to let them have their relationship... [this was especially true when] we got his diagnosis, lot of people were in denial in our family. They'd only met him once or twice and he seemed very typical. And he did until 18 months and the moment we got the diagnosis it was a total shock to us. You know we got over that denial stage much more quickly [than they did]...it's kind of awkward. They don't see us very often. We don't know how to tell them how to interact with them...And yet at the same time they're trying.

She told me extended family members asked, "how can [the doctor] tell he's so young. But he talks, but he hugs, but he..." She said "And he does, he's very affectionate and he does have some language." I asked Caroline to elaborate on the extended family's source of denial and why they didn't believe he had autism. Caroline looked down at the photograph for a long moment before she answered. Caroline shared that some of her own family's denial of Nathan's needs resulted from their misunderstanding of autism. Caroline has a step sister with "classical" autism and is very significantly impacted by its symptoms. Caroline shared that she was also diagnosed with autism around the time of her son's diagnosis.

Later Caroline shared another example of extended family's reaction to Nathan's autism. She said she and her husband are thinking of having another child. She always wanted three or four children. She told me that her mother-in-law asked her "if it would be fair to another child" because of Nathan's autism and his needs.

CAROLINE: [The] funny thing is that she has six kids. I was like, "Why would you go and have [six kids]?" They're Mormon. They planned on four, like "why would you have five and six? [Was it] taking away from your other four?" Like that doesn't make sense [either]. But [then] she's like, "I really like the way that you're thinking." Like I mean she thought [I was right].

Towards the end of our first interview, Caroline was told me about photograph C2 (Figure 5). She said that she chose the photograph because it depicted her whole family. 
Caroline said she picked this picture because it's not an "autistic thing" or a "mommy is autistic thing" but "But ...we are a family and we have the resources that we need."

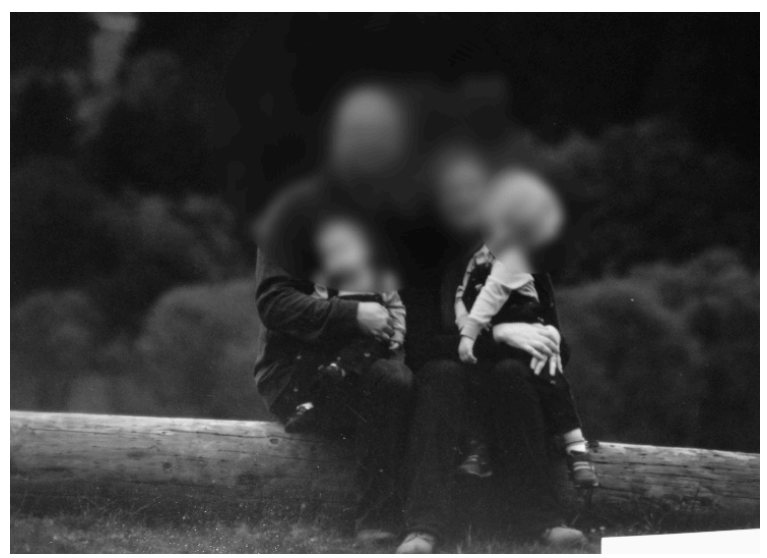

Figure 5. Photograph C-2. A photograph of the whole family: mom, dad, and both boys. They are posing outside on a $\log$ in the woods. Nathan is kissing Caroline on the cheek.

When I looked at the photograph I felt like I understood what Caroline meant. In the picture both parents are smiling. Caroline is holding Nathan and he is kissing her. His baby brother is held securely in dad's arms. It looked like a young, happy family. Caroline shared with me that their family works because "everyone gets what they need" or what she and her husband have available to give. When Caroline's in-law expressed concern over whether Nathan's brother was having to sacrifice for all of Nathan's needs." She was adamant in her conviction about what family means. She responded to her mother in laws query "No. We're just family...And sometimes you know our resources as a family are limited to what they are."

\section{Early autism symptoms \& diagnoses.}

Social interaction and early autism. Our conversation meandered a bit and the topic of social interaction and autism came up. I shared some of the dominant theories on 
social development and autism. I asked her if she agreed with a theory that children with autism are averse to social interaction or if she felt sensory issues made social interaction difficult. I explained the Social Motivation Theory (Chevalier et al., 2012), which theorizes that infants with autism have disruptions in the bias to orient to social interaction. She had a strong reaction to theories that purport people with autism don't want nor seek social interaction.

She picked up picture C-3 (Figure 6) and slapped it back down on the table. "Look at this" she said "[lack of] social orientation, my ass!"

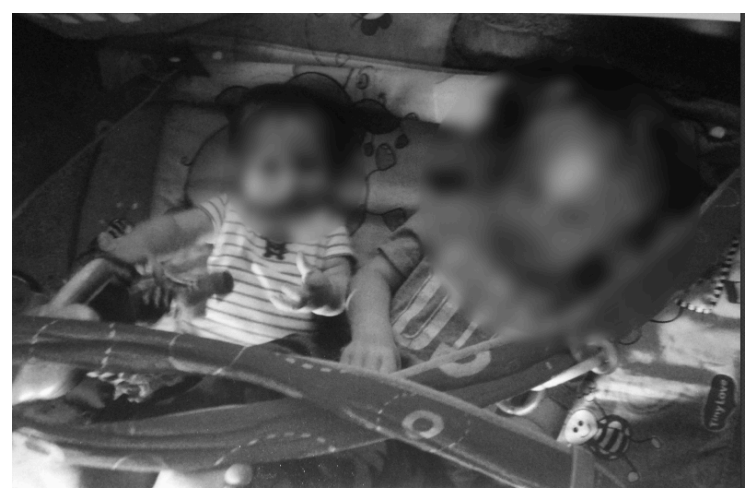

Figure 6. Photograph C-3. The photograph depicts Caroline's son Nathan with his infant brother under a baby tent toy. Nathan crawled over and lay down next to his baby brother.

She explained social interaction and autism this way:

CAROLINE: It's not that they're not having social interactions. They're interacting the way - when your brain is structured how an autistic child's brain is structured - [they] are interacting with the world the way that the world occurs to [them].

In the photograph Nathan had crawled under an infant sensory toy to be with his baby brother. Caroline pointed out that because of the infant's unpredictability it was often hard for Nathan to tolerate their interactions. The photograph represented how 
Nathan sought interaction with his brother and in his own way.

CAROLINE: Playing like this is social. He got under there. He doesn't particularly like his brother but he's next to his brother. And ... he's tolerating. You know his brother's playing with something over here. He's playing with something over here. It's parallel play but you know it's social. He could be in the other room. He could be doing any other thing.

Caroline Shook her head and looked down at the pictures for a moment. Then she selected Photograph C-4 (photo redacted). The photograph was taken at Nathan's preschool. In the picture, he and a classmate are facing each other. The playground is in the background. Nathan's arms are on the other boys shoulders in a show of affection., an approximation of an embrace.

Caroline held the photograph so I could see it. She told me his hugs don't look like other people's hugs. He "sees you, grabs you and hugs you. Then he walks away." She laughed at how cute he was. "It's so funny....He's incredibly social." She grew serious for a moment. I could see it was important for her that I understood the difference between "not wanting social interaction" and "socially interacting differently." She told me it makes a difference that others understand that he's delayed in that area. With supports he can interact and be successful. She said Nathan is beginning to interact socially with his peers and figuring out how to play with other kids. His preschool is "giving him tools," and "equipping him with ways to figure it out." She said Nathan "needs someone there supporting him. He's so calm and he does great with it. But yeah...he's a very social creature."

Road to diagnosis. When Nathan was about 18 months old, a teacher conducted an Ages and Stages Questionnaire (i.e., a developmental screening for young children) 
and Nathan had red flags in six categories. She told me, "we were like 'oh my God.' $\mathrm{He}$ is not even on the charts for three of these six categories."

The teacher also told Caroline that she was concerned that Nathan didn't seem to hear her. When Caroline called her pediatrician for a referral he suggested Caroline wait until 24 months to bring Nathan in since he'd just had his 18-month well-baby check. Caroline didn't agree. She knew something was wrong.

During that time Caroline realized, "He still didn't seem to understand things and he only turned to look at you about half the time when you called his name. So we thought maybe he did have a hearing problem." After that Caroline went against medical advice and started seeking other medical help.

CAROLINE: So I went behind his pediatrician's back and called OHSU and said "can I get a hearing test?" ...So we got a hearing test from the audiologist...At the same time I kind of looked at her and [then] said "WE-NEED-HELP!"

Raising Nathan in a neurotypical world. Caroline told me about how Nathan's accommodations have become a natural part of their daily life. In a sense, as parents Caroline and her husband pick their battles. Caroline told me this is a little hard to explain to the outside world where children interface with rules for neurotypical children.

CAROLINE: I had to take him to an appointment with me one day and he was flicking the lights on and off and it didn't even occur to me because at home it's just one of those things he likes to do it. We can't explain to him that 'no we do it in certain places.' ...The doctor was like [raises voice impersonate] 'we don't do that.'

Autism services and family life. Caroline felt strongly that parenting and navigating services cannot be separated when you have a child with autism. She told me that parents spend a great deal of time trying to get services, coordinating between 
service paradigms, and taking their children to appointments. She described it as "cracking the whip" and a parent always has to be on top of it.

Caroline's said her experiences with special education EI providers have been phenomenal in terms of the people and the philosophy. The problem is that EI isn't funded so the services ineffective. The medical establishment on the other hand is highly specialized and Caroline does not see it as the medical system's role to determine services to address the family's goals. Caroline felt that there needed to be a coordinated system of care that is "family-focused." She wasn't sure who would be responsible for coordinating services but it should be such that parents' sign consent and all services work together.

Private preschool. Caroline selected another prompt and photograph number C4. The photograph is taken during a preschool activity. About 15 children are seen standing around a long table where activities or lunch might take place. Some children are in the foreground. Nathan is in the back of the room and a teacher is kneeling next to him. She picked up one of the pieces of paper with the prompts and read "select a photograph and tell me about the people in it and their relationship with your child." Caroline told me she loves the preschool and feels the community is very accepting of Nathan and open to her advocacy on his behalf. She has been very open with the families about Nathan's autism. I realized as she spoke that advocacy and accommodation are a part of all of her interactions with the world as they relate to Nathan. Nathan is successful in part because of how in-tune she is with him and his needs. She seems to know she will be successful through a combination of accommodations and the natural course of 
development. Caroline understands the neurotypical world and the autistic world and bridging the two is integral to her parenting.

Challenges and obstacles. About halfway through our first interview Caroline looked back down at the photographs spread across the table. She grew serious and stared for a moment at picture number C-5 (Figure 7) She picked it up slowly and kept her eyes on it for a moment.

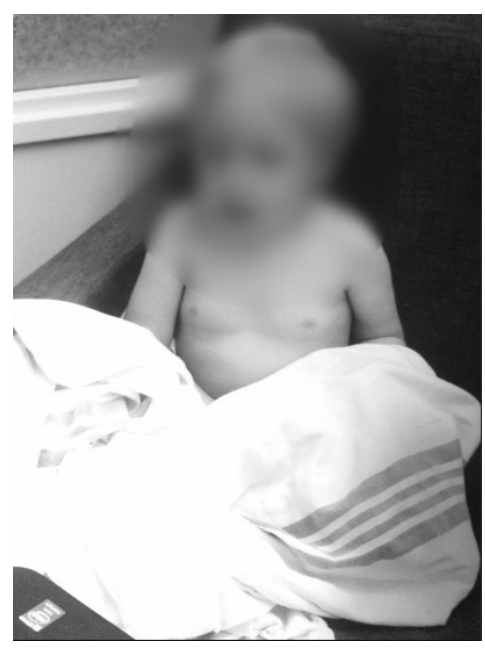

Figure 7. Photograph C-5 depicts Nathan immediately following ear surgery. It represented the exhaustion and angst Caroline sometimes feels over how much her son has to go through and how heart wrenching it can be.

Caroline told me about Nathan's medical issues. He has complex and significant medical issues including vitamin deficiencies, ear infections, tummy problems. He also has significant dental problems and has had to undergo fillings and crowns on many of his teeth. She said:

CAROLINE: He's been under general anesthesia more than his dad and I combined... The poor kid...He just generally has digestive problems...he has deficiencies and we give him twice the amount [of vitamins] you would give a normal adult and he still doesn't absorb them - we don't know the nature of his gut problems... [And] he has ear nose and throat [problems]... moderate hearing loss. He got tubes in his ears, he could hear after that...um....and just like he has 
four crowns and eight cavities and all his teeth are capped.

Caroline explained that in photograph C-5 Nathan is in the recovery room after surgery to place ear tubes.

CAROLINE: This is...sad...Of course my child is wonderful and of course I love parenting him but there are hard times too. There's both. It's amazing and it's heart wrenching and this is more of [the] heart wrenching, you know? Just how difficult it is and... he can't tell us what's going on and we can't communicate to him "no leave your IV in." Even just the look on his face... at this point I had to take off half of last year just to get him back and forth to doctor visits and ER visits. We had a really rough year last year because he can't tell us and we're constantly taking blood and we're constantly going to the ER and he's throwing up blood...

I sensed how significant this photograph was to Caroline. I reflected on it after our interview. It was a lot for a parent to handle. It wasn't just his autism but the way it intersects with all the other challenges life has to offer. I also realized how powerful photo-elicitation methodology was in translating her lived experiences. As she stared at the photograph she said "that expression on his face is like..." she paused a moment, "that's the expression I wear on my heart." The photograph captured the look on her son's face, his pain and hardship. She wears that on her heart.

Before ending our first interview Caroline stressed a point she had made several times during our conversation.

CAROLINE: this is like the third time I've said it but [autism] is such a huge part of our life. It's so central to everything that we do and how we figure out how to interact with our kiddo.

I reflected on this statement quite a bit. Caroline's was my first interview and I had expected that there would be parenting lived experiences that stood out independent from the autism or treatment. I imagined the essential theme of parenting was related but 
distinctly separate from the essential theme of navigating services. When I looked back over my notes a few days later, I considered what Caroline had shared with me at the beginning of the first interview.

CAROLINE: [Autism is] front and center. It is the elephant-I mean space-wise in this teeny tiny room, it would be the elephant literally in the room. There's so much. It influences...absolutely influences every little thing our family does.

The second interview: A deeper exploration of service experiences. Caroline and I met for the second interview in her home. I immediately recognized evidence of young children. It shared characteristics with other family homes I had been to and of my own home when my daughter was in preschool. Low shelving lined the walls of the living room where we sat. The shelves had baskets full of books and toys. The refrigerator in the kitchen was covered in letter magnets. And the bathroom had buckets of bath toys. I knew from my early childhood training that the selection and arrangement of toys would support healthy child development.

We sat on the couch together and returned to our conversations from the first interview. We again laid the photographs in front of us on the table. I offered her an opportunity to elaborate or clarify anything we discussed in the first interview. I followed up with several open-ended questions to explore service experiences more deeply. Most of the second interview focuses on autism services for Nathan.

Caroline's experiences developing an IFSP, a "road map". Nathan will be starting kindergarten next year. He is currently enrolled in a number of service programs and Caroline is skilled at managing and coordinating them. She now knows who to call, when to apply pressure, and when to let things go. She navigates the systems fluently. In 
a sense, her journey from early concerns to autism services has ended. She is now looking forward after having made it to the through the challenges of seeking help and finding AUT-EI.

In addition to managing and navigating services, Caroline understands the importance of communication between systems. All parents expressed that there is no mechanism for that in the current service model. In response Caroline uses the IFSP developed by the educational district as a "road map" for all providers. She works from the IFSP goals with all providers and sees to it that their work is done towards the goals as laid out in the IFSP. She said" --everything that we do we take his IFSP. [We bring it] to his therapists. We say 'these are the things that we're working on.' And so they typically write the therapies around [the IFSP goals]."

Early intervention services through the educational program. Caroline was dissatisfied with the educational services but not the educational specialists with whom she worked. She recognized that because there is a lack of funding, the services are ineffective. She explained her experience when Nathan was under three:

CAROLINE: Before his autism diagnosis he qualified for EI social and communication. He was like a negative 12 out of 100 you know [laughs]. He's severely delayed. You have to be 2 standard deviations below. So he qualified for 8 visits from the coordinator and 8 visits of speech a year and it's supposed to be provided by that [EI] coordinator. What kind of coordinator do they send us? An occupational therapist!... Which by the way turned out to be the biggest blessing in disguise because it turned it $-\mathrm{I}$ think most of his issues are sensory and dealing with sensory issues has been the biggest issue but we were entitled to speech-we never got speech-

According to Caroline if the educational programs had the money the specialists could do their jobs and Caroline believes they would benefit her son. However without 
the funding Nathan is not getting the service he needs and thus the services he gets from them are "absolute and utter shit. It just is." " The only reason she and her husband enrolled Nathan in the special education system was as an exercise in the IEP process in preparation for kindergarten.

CAROLINE:...The only reason we're doing it at all—with this minimal amount of services from early intervention, it's an exercise for us to get ready for actual IEPs once he goes to school because we do plan on sending him to school and so we do have IFSPs and we do have services but they're not even—like we're not even getting what we're entitled to.

She saw early intervention as "practice" for school age services and working with the education system. All participants in this study expressed this sentiment. She also explained that even if the services were delivered they would not be effective because of the current model's limitations:

CAROLINE: We didn't push it...[but in home EI specialists] don't spend enough time. Once a freaking month or less than once a month...like 8 appointments does not equal 8 appointments because you have to orient yourself to the child and get the child comfortable with you to even do any intervention. And of course they're not -they're not in the same space as they were the last time you saw them. You know they've grown and developed or...um they don't remember from the last time....So it's completely an ineffective model of services

Medical and services through private insurance. As our conversation about Nathan's services continued, Caroline picked up photograph number C-6 (Figure 8). 


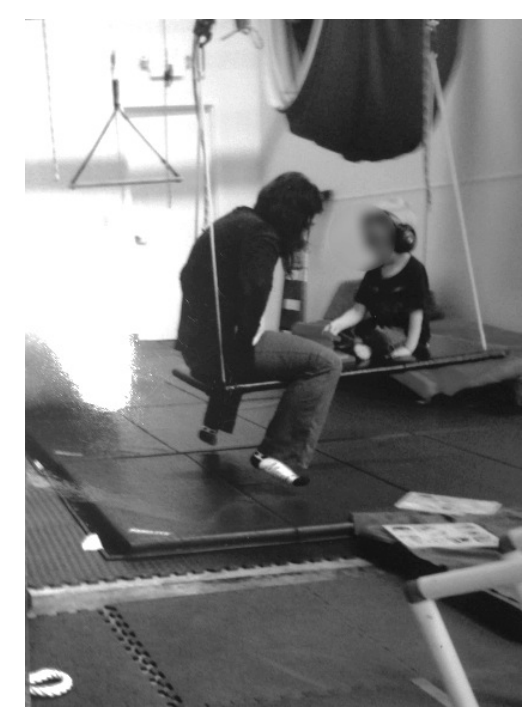

Figure 8. Photograph C-6. The picture depicts Nathan with his occupational therapist. They are sitting on a padded swing, working. He has on a pair of headphones and he is engaged in therapeutic listening, which calms him and helps him attend to therapy.

Caroline found an occupational therapy program through her family's medical insurance. Nathan's services include occupational therapy, private preschool, and the limited services provided by special education EI. Caroline felt the occupational therapy has made a "phenomenal difference" in Nathan's life. It addresses his sensory and auditory processing issues However, Caroline told me that getting and keeping this service has been a big fight with Medicare because it is a specialized program called therapeutic listening. It involves wearing headphones that play sound and music at different frequencies and has "a calming effect" on Nathan and he can "process things differently." She expressed frustration over the constant fight for services. She said, "All my life — all I am doing is constantly keeping people accountable for what they're supposed to be doing with regards to my son."

CAROLINE: It is a constant battle because you're constantly approaching these people and saying 'ok you're supposed to be doing this and you're not. Or 'what 
about this' or you know 'you dropped the ball' or 'like this.'

Summary. Caroline and I wrapped up our interviews and I encouraged her to contact me if she thought of anything or had anything to share. I thanked her for the interview. Following the second interview she sent me a long email with clarifications about our conversation related to EI services.

\section{Narrative 2: Emily's Lived Experiences}

Meeting Emily. Emily learned of my study through an autism mom's list serve. After the initial screening Emily expressed that she felt the study was important and wanted to help. She had met the study's inclusion criteria. Emily worked in administration for a large medical establishment. She expressed an interest in returning to school and perhaps studying education some time in the future. She originally grew up in Utah and had moved to the Pacific Northwest with her husband. She had been married since college where she met her husband. She has a nine-year-old boy, a six-year-old girl, and a three-year-old boy. At the start of our interview she told me the two older children were at school and that her youngest was in Applied Behavior Analysis (ABA) therapy.

Emily and I met in her home for both the first and second interviews. Emily, her husband, and their three children live in a single-family home in a suburb of a large metropolitan area in the Pacific Northwest. I immediately felt at home and welcome. Emily was outgoing and attentive during our interview. I had the feeling that she had a lot to do with work, three kids, and managing the household. A plumber came through the house during our first interview and Emily fluidly moved between our conversation and giving instructions to the contractor. I very much appreciated her making time for our 
interview. As we sat at her dining room table with her large dog on the floor near us, their family life was evident. Pictures of the kids hung on the wall and the furniture was comfortable.

I asked Emily if she was ready to begin the interview. Prior to the interview I had asked Emily to gather the 10 photographs for the interview. She said she had gone through her digital photos and selected 10 and they were on her phone. Although discussing photographs on a phone screen was a different experience than having the pictures laid out before us, it was effective. I placed the written prompts around the table. She chose 'tell me about the people in it.'

Family life.

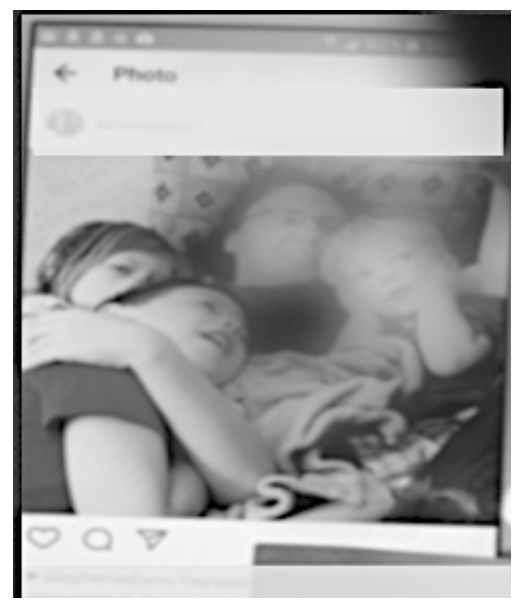

Figure 9. Photograph E-1. Emily's sister with the three kids. They are all exhausted from a day at a family wedding and cuddled on the bed together.

Emily looked into her phone screen and had photograph E-1 ready for us to discuss. The photograph is depicted in Figure 9. She smiled for a moment as she looked at the picture, then she turned the phone so I could see it. It was a photograph of her sister with her three children. They were lying on a bed in a hotel room cuddled up. She looked 
down now and then as she told me about her sister's relationship with the family.

EMILY: These are my three kids and this is my sister ...I'm from a family of 5 kids my little sister - was adopted and she's like my best friend and she's also a special ed teacher... She's kind of like walked me through everything that has happened to us. It's so nice because she's been like 'ok this is what's going to happen. This is what you're going to need to do. You need to call early intervention, blab la blah." And she's fantastic to my children. She's also my children's best friend. She's on the will that if anything happens...

The second photograph E-2 also centered on family life (Figure 10). It was a photograph of the three children on a family holiday trip to a water park hotel, a yearly Thanksgiving ritual.

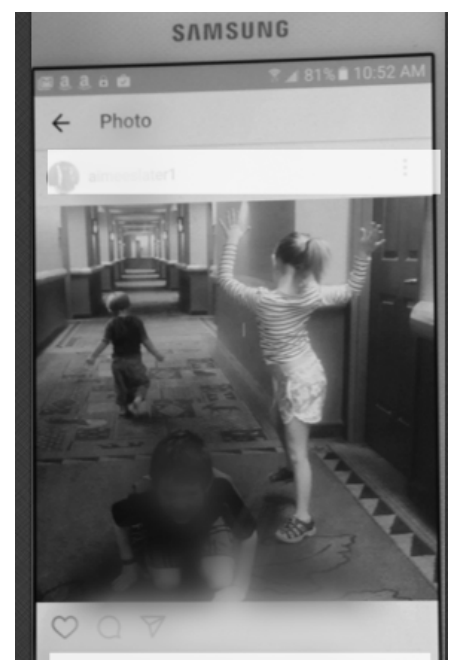

Figure 10. Photograph E-2. Thanksgiving holiday at hotel. Emily chose this picture because she felt it captured the personality of each one of her children.

As she shared the meaning of the photograph to her, I thought of how each member of their family has a unique personality and how this photograph reflected a love and affection for each of the children.

EMILY: My in laws they're retired and they live in Utah. And my familythey're in Texas so we don't have any family and no brothers or sisters or anything, in the area. So every year at thanksgiving we go to [water park]. They're in the hotel and this picture expresses their personalities very well. Because my son - my oldest always trying to be the center of attention- 
RESEARCHER: and there he is right there in the middle of the photograph. EMILY: Oh yeah. He totally, always has to be the center of attention and my daughter is always dancing so it is just kind of funny because she's dancing and he's always saying '[to his sister] don't dance you look funny. You look weird.' And Joshua [the youngest with autism] is always running away...They call it elopement? He has a huge elopement problem so we actually have [an alarm on our door]. I turned it off this morning .... and a we have a chain, chain because one morning we were upstairs on a Sunday and [Joshua] was across the street running in circles and we had no idea he left...He just leaves. all the time.

Family characteristics, likeness.

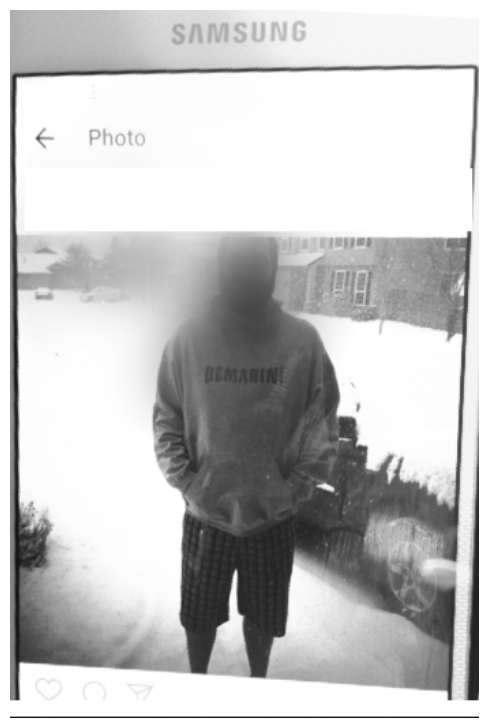

Figure 11. Photograph E-3 of Emily's husband standing in the front yard on a snowy day.

Emily looked back down at her phone then showed me picture E-3 of her husband (Figure 11). The picture depicted her husband outside in shorts. There is snow in the background. He is wearing a hooded sweatshirt and smiling. Emily told me that the picture was taken on a snow day and her husband had slipped and broken his wrist. Joshua was only 6 months old. Emily insisted that her husband go to the hospital and have his wrist checked. She had to take that Saturday off from work because he couldn't hold the baby with his broken wrist. Emily spoke of her husband with affection and 
humor. They'd been together 22 years. She described her husband to me and half joking wondered if he was "on the spectrum."

EMILY: It's funny because like my friend that has gotten me into [autism program], she has a son that's on the spectrum and she's like (joke whisper) "I always wonder about my husband." And I'm like (joke whisper) "Me too." (laughing). My husband has horrible social anxiety. He has good social skills when he like forces himself but he's like totally an engineer nerd...You know what I mean?

RESEARCHER: ...is he super involved with the kids?

EMILY: Oh yeah. He's very involved with the kids but he doesn't have a lot of [close friends] — he has like one or two close friends. He doesn't socialize a lot. Social outings are very hard for him unless it's with like really close friends or things like that. I'm like (joke whisper) "I always wonder" not that I would say anything...(laughing)

EMILY: He's shown a lot of like 'nobody talk to me. I'm going in my corner...[and] do my engineering work [and] leave everybody alone.'

Emily once told her sister she wondered if her husband was on the spectrum. Her sister told her "that's not going to change anything." But Emily told me it makes her feel better.

Later in our interview Emily shared another photograph (E-4, Figure 12) that represented family to Emily. It was a picture of the kids at Christmas all in their Star Wars pajamas. To Emily this seemed to represent her family, all together at Christmas, all loving Star Wars with their dad. 


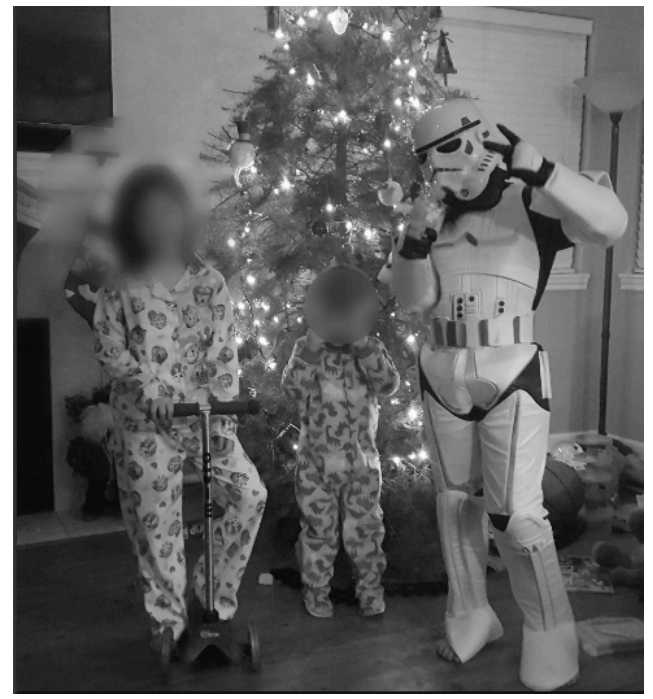

Figure 12. Photograph E-4 of Emily's children on Christmas morning. The oldest in a Star Wars Costume. Joshua is in the middle between his older sister and brother.

\section{Extended family.}

Emily and her husband united. Another aspect of family life that Emily and I discussed was extended family and how both sides felt about Joshua's autism. It was during this conversation that I saw how united Emily and her husband were and also how committed they were to bridging Joshua's needs with extended family members' expectations. It was my impression that in some ways advocating for Joshua reinforced the bond between Emily and her husband. Emily said both sides of the family were "very against us having anything to do with having Joshua on the spectrum.” But Emily and her husband were united reiterating "we think [getting help] is important for him." She told me that she's heard that having a child with autism can break up marriages, but it has "brought [ours] closer together."

Bridging extended family relationships. Photograph E-5 (Figure 13) depicts all three kids sitting on a couch with quilts Emily's grandmother made for each of them. 
Emily told me that it had been very important to her grandmother to finish the quilts for the children. Emily's grandfather had just died but she worked on the quilts so she could give them to Emily's children when they came the weekend of the funeral. Emily felt she'd inherited some of her grandmother's resilience, stubbornness, and strength. Emily's grandmother had originally been skeptical but now acknowledges Joshua's autism and also Emily and her husband advocacy and support. To me, this photograph represented how the extended family came to understand and accept Joshua's autism. I recognized the themes of family connectedness, identity, and belonging.

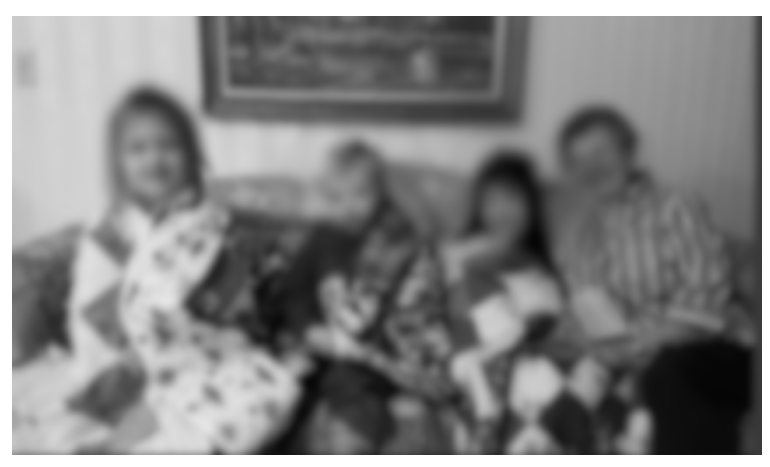

Figure 13. Photograph E-5 of Emily's children with their great-grandmother, each covered in a quilt made especially for Joshua and each of his siblings.

EMILY: It's my dad's mom. I think she is probably where I get a lot of my stubbornness. She was a WWII refugee from Denmark, and so she came at fifteen years old and didn't know any English...At first, she was like "doesn't every kid have Autism?" And now she's like, "I'm really glad that Joshua has the advocate you are," and I'm like "that's really sweet." She's really sweet. Anyway, she made each of them quilts and it was really sweet, and I had to get a picture.

Our conversation turned to extended family members' doubts and skepticism about Joshua's autism. Some thought Emily and her husband "were making a big deal out of nothing." Her mother who is a registered nurse attributed Joshua's early autism symptoms to earwax, saying, "Oh you didn't clean out his ears very well." Emily felt her 
mother's comment was an example of how often people "don't believe you" or "say 'it's nothing." Her mother in law had told her:

EMILY: ““...Back in the 70s when I was raising a kid no one really cared about that kind of stuff. Everybody's kids didn't say much and that's fine. You just put them through speech therapy-

Emily and her husband also dealt with more subtle criticisms. One of her

grandmothers still says things like "well I think it's nice you're getting him some special attention." Emily said "That's what she calls it his special attention. I'm like..ok. that's great." Emily's mother in law told her, "You know, your husband couldn't say his R's for a year." Emily said she wanted to respond, "Ok not only can Joshua not say his R's --or any other letter of the alphabet."

Early symptoms and diagnoses. Emily told me that at about 18 months she started noticing that Joshua was regressing and that was when she "suspected autism." She described her early concerns:

EMILY: [I suspected autism] probably a year, or like 18 months. I knew he wasn't picking up skills or he could sing a whole song and then he would forget it. Like he would sing Itsy Bitsy Spider... Twinkle, Twinkle was his favorite and he would sing like the whole thing and then you'd try to get him to repeat it and he wouldn't do it...He was losing skills. You know what I mean? And you could tell. I was like this is-

RESEARCHER: oh he would like know it for a couple months or something and then it was gone?

EMILY: Yeah. So he was losing skills and his I was like 'that was really weird.' And he would do something really cute or really smart and I could never get him to repeat it..and I was like this is so frustrating because as I'm trying to take a million pictures of my child, I can't because I can't get him to do anything...

Emily said Joshua "flunked" his 18-month screening by the pediatrician but the doctor felt there was nothing wrong with Joshua's development and that they should wait and see. At that time Joshua was nonverbal and had problems with fine motor skills. $\mathrm{He}$ 
also had difficulty with eye contact. Emily began researching on the internet and Facebook groups. At the 24-month visit, Joshua did not pass the M-CHAT screen but the doctor still felt that it was very unlikely that Joshua was autistic. "Because," the doctor said, "he almost passed the M-CHAT." By the time Joshua was two and a half Emily felt they really needed a referral to a developmental pediatrician. A diagnosis by a developmental pediatrician was required by her insurance in order to receive autism service coverage. The pediatrician reluctantly agreed to the referral. She described the day she insisted on a referral for Joshua.

EMILY: I took one of my kids in sick when [Joshua] was two and a half and I was like 'he's still not talking and he's getting special ed things through the schools and the schools are saying he needs to be tested.' And so the doctor is like 'I'll put in a referral but I think it's a waste of time'...because he's a really old fashioned doctor-he doesn't. to him he thinks it's over diagnosed and over treated. He thinks 'oh everyone thinks their kid has autism.

I felt a sense that Emily was forging her own way to getting the help that she intuitively sensed Joshua needed. The behaviors she was seeing in her toddler son were consistent with the literature on emergent autism. For almost two years Emily was met with doubt and disregard for her concerns over autism. Family members and medical service providers told Emily she was overreacting. Her pediatrician counseled "Joshua has other siblings that are just fine...Y Your kids are really intelligent.” Emily tried to persuade him, "he's also really intelligent but he struggles."

Even when Joshua failed two developmental screenings, the pediatrician said he did not want to jump to any conclusions. The doctor did not accept her answers to the parental questionnaire on the ASQ screening. When Emily responded never (i.e., on a 4 point scale from 
always to never) to behaviors she observed in Joshua, the doctor did not accept her response and changed it to sometimes. The doctor explained his reasoning, "well I saw him do this once [in the examination room] so that counts." He also told Emily, "you're saying never and maybe sometimes you're just not seeing it." Despite what she'd observed with Joshua's development and her intuition, when the doctor disagreed with her, she deferred to him. She trusted his professional opinion “...they're the pediatrician and we're just the parent....Sometimes you take something somebody says as bar none. It is what it is."

Around that same time Emily felt the educational EI providers were hinting that Joshua might have autism but didn't come right out and say it. She felt they weren't allowed to indicate they thought he had autism so they kept encouraging her to have his hearing tested. Joshua had already flunked two hearing tests. When Joshua was 2 years, 5 months she came right out and told the early interventionist she thought, "Maybe he has autism?" According to Emily the EI professional "had to wait for [the parent] to say it." The early interventionist told her "You should look into that." Emily felt that they do that on purpose because they don't want to scare parents away.

As she spoke to me I felt that the advice from both the doctor and the early interventionist was inconsistent with what I understood best practices to be. I tried to bracket out these preconceptions and focus on gathering more information about Emily's experiences. This was difficult to do during this portion of the interview because my understanding was that pediatric young child screenings are meant to identify children at risk and to refer them for an evaluation. I did not think the protocols were to "wait and 
see" if a child scored positive for autism concerns on the MCHAT. Further, it was my understanding that early intervention providers could talk with the families about options, information, and recommend evaluations if warranted.

As Emily continued sharing her early experiences with service providers I wondered if the providers themselves possessed knowledge about emergent autism symptoms, diagnosis, and intervention. Perhaps, the research on early markers and early assessment is not widely known in related fields of practice. Emily told me she mentioned Joshua's autism to a special education teacher at the school her other two children attend. The teacher was surprised that there were evaluations for autism at such a young age. The teacher told Emily, “'I've never heard of the two year olds getting diagnosed before. Those kids [with autism] aren't diagnosed until 7 or 8 , like when they get in the classroom and the teachers notice there's something off [about the child]."

\section{Raising Joshua in a neurotypical world.}

Navigating Services. As we talked, I got a sense of the prominent role Joshua's service coordination played in the family life. Emily and I talked in more detail about the delay in services for Joshua. Mainly, the medical side of services has long waits and can delay autism diagnosis by months if not years. Between the hesitation on the part of Emily's pediatrician and the wait time for referral and scheduling the evaluation Emily had to wait over a year and a half after failing the autism screening at 18 months.

Emily described what she viewed as a series of obstacles in a parent's way of obtaining a diagnosis and services for their child with autism. According to her, the problem with the medical system is that you can't get insurance to cover services unless 
you receive a diagnosis. You have to be referred by a pediatrician for a developmental pediatrician or other specialist who can do the evaluation. There's a long wait for an opening, often six to eight months.

EMILY: ...In August, he had his well-child, and then they said "okay, well if he doesn't get better, email me, "and so I talked to him again in October when one of my other kids was sick, and he was like "okay, I'll put in a referral to the developmental clinic, but don't worry about it." They didn't call me for two months and then when they finally called me in December, they were like we're booked out until May 12th, that's our next available. And so they're like we can... like they do a phone assessment first, and so the doctor called me in January sometime, and she said, okay, we definitely need to get him in, and then May 12th is the next one. I said, "okay." She said, you can call for cancellations, so I would call weekly for cancellations and they said "we only do this once a week, quit calling."

The problem becomes even more stressful when the importance of early intervention for autism is stressed and a parent can't access services. She said "they're the ones that have to pull the trigger. Put in a referral for services." It's not much different with the early intervention educational programs. They "make you jump through hoops before you get all of the early intervention stuff."

We also talked about some of the issues associated with the medical services themselves, once a diagnosis is made. Healthcare costs associated with autism treatment are very expensive and without insurance would not be possible for many Oregon families. An autism diagnosis opens doors to coverage for more and specialized services like occupational therapy, speech therapy, and applied behavior analysis. If Kaiser didn't cover Emily's ABA for Joshua, it would be " $\$ 12,000$ a month for 20 hours a week." Applied behavior analysis programs are not offered through the educational program, only through programs that accept medical insurance. 
Challenges and obstacles. During the last half of the second interview Emily and I talked almost exclusively about the amount of time and effort she dedicates to coordination for Joshua's autism services. She described many obstacles and the need for constant monitoring and management of services. While the educational programs lacked funding for the appropriate level of services, the medical establishment also had resource problems. Emily said she had to make accommodations to daily life to keep Joshua safe and so he could participate in typical childhood activities. She shared that they have had to put an alarm and a chain lock on the front door because of Joshua's "development problem."

Stress over possibility of losing services. Emily told me that ABA services are in shortage and the demand is high. There are strict attendance requirements. At the agency Joshua attends, a child is dropped after 3 occasions of being more than 15 minutes late. Emily hired a baby sitter out of their own pocket, paying $\$ 15$ / hour, 25 hours a week.

EMILY: [The babysitter] came in the morning and she would get my kids on the bus, and then she took [Joshua\} to ABA She studied for four hours at the library [while he was in ABA] because she was a student, and then she would pick him up and bring him home.

RESEARCHER: It would be five hours a day?

EMILY: Yes, and [ $\$ 15$ an hour] is the going rate for special needs providers... For me, it was fine, but I just kept burning through them really fast, and...so after a while I said, this is just not even worth it. It's not worth my time, and mostly my stress.

Even with a babysitter, there were days when Joshua risked being late. It was so stressful for Emily that she switched from working days to the night shift. Now Emily's husband is at home nights with the kids, and she is there during the day. It has cut into their family time and she often feels guilty that she can't spend more time with the other 
children. Sometimes her son waits up late for her. Emily expressed that both of her other children have to sacrifice for Joshua's needs. She spends so little time with the kids because of her work schedule. Sometimes her son will wait up for her when she returns from work at 10:00. She said "he talks my ear off for a good half hour, forty-five minutes." He told his dad, "I really like staying up for mom because I really like to talk to her."

Worry over missed early intervention services. At 24 months Joshua was referred for speech services. She found out later that he should have been referred to an audiologist after failing 2 newborn screenings so he had missed two years of therapy. Emily felt he should have been referred to an audiologist at 12 months and received services weekly. She worried about the critical periods he missed and the effect on his development. For that reason once he was diagnosed she pulled some strings and got Kaiser to give him two sessions a week rather than the originally prescribed once a week. She felt her ingenuity had paid off as she saw Joshua made significant gains as a result. However Kaiser caught on and said she couldn't do two sessions

Interview 2: A deeper look at service experiences. Emily and I emailed each other back and forth to schedule a second interview. The holidays delayed our meeting a week. There was also a number of snow days and both of our families had the flu at during that time. We rescheduled several times and finally met again in January, two months after our first meeting. Again we met in her home. When I arrived Emily was waiting outside with her older children for the school bus. They had missed it as it passed on its way into the neighborhood and were waiting to be picked up on its way out of the 
neighborhood. After the kids got on the bus Emily and I went inside and Joshua was still home. Joshua hadn't been picked up for preschool yet and so he spent the first few minutes with us. Their dog Star was asleep on the living room floor.

Emily ran upstairs to get Joshua's backpack and school supplies ready. He sat with me and I showed him the microphone. I said "hello, hello." Into the microphone. Joshua laughed and said “Hello. Hello. Scary Grandpa!” Emily came back down and related that Joshua associates the phone with one Skype call from his grandfather. His grandfather playfully had pretended to be a monster. She asked him to get his water bottle and told him the bus would be there soon.

To me the scene was reminiscent of any family with children, getting them off to school. The only difference I noticed was perhaps more verbal scaffolding and support with the routine with Joshua. Emily elaborated on his 'scary grandpa' story and reminded him several times to get his water bottle and snacks for school. She was affectionate with him and seemed delighted with his silliness over the family joke about grandpa. He was off to school shortly after the other children and we began our second interview.

Once we settled in for our interview, Emily shared that Joshua attends ABA 20 hours a week and special education preschool two days a week. She said liked the ABA program because it provides detailed reports of Joshua's daily activities in the program. They also communicated with her about the things he's learned. Emily felt that of all the services the ABA was the most beneficial for Joshua. Emily also told me how ABA therapy has made Joshua more verbal. At first it was just requesting but now he's begun sharing interests. 
EMILY: I love [ABA]...because I feel he's making so much progress...He says things... It's funny because they try to get him to vocalize his eating now. Like they try to get him to verbalize what he is feeling and stuff. And he'll come home and have a cup of milk and he's like 'Mm, that's delicious.' I'm like, where did this come from?

She recognized how hard it would be if she could not afford it. His development would be negatively affected. Since he's been doing ABA, his language has improved dramatically. The program systematically teaches language and social skills. He goes in the morning once a week and every afternoon. They also provide frequent progress reports where she can see the data tracking his mastery of skills. The ABA was also improving Joshua's ability to communicate at home, not just his requests but also to share what he is experiencing. Emily's insurance covers the ABA, otherwise, Joshua would not be able to receive it.

EMILY: So it's private insurance, but super expensive...He gets 20 hours a week right now. They're trying to re-up it for 30... I guess Kaiser has a contract where it's $\$ 75$ an hour, but my friend who has United, United pays $\$ 12,000$ a month....[at] the same place. I mean, Kaiser has some kind of contract where it's cheaper, but $\$ 12,000$ a month, because her son goes for 40 hours... and of course, parents pay a portion of that.

Early special education programs through the Educational Service District. Up

until this point Emily had mainly focused on services through the medical side of things. I asked her about the services she receives through special education. Emily told me that Joshua attends a preschool program twice a week through the educational service district.

EMILY: He likes riding the bus to school. He's not that verbal so when I say "how was school?" He'll say "ride the bus!" and that's like all he can say. I have no idea [how he's doing in school]. The teacher does not communicate so I have no idea if he likes school or not.

She said the teacher does not keep in contact with her. She recently asked the 
teacher what Joshua was doing in school and the teacher related the classroom routines (i.e., circle time, snack). Emily asked about specific behaviors like eye contact and verbalizing. The teacher said she didn't know. Emily told me about the school progress report:

EMILY: It's a Special Ed class for preschool. And it's funny, his report card came home and all of his goals were not met, and they were like 'his goals are not met because he does not come to class more than twice a week.' I was like, 'okay, whatever.'

The school speech therapist also could not comment on Joshua's school performance when Emily asked. She told me she figured it's not hurting him.

Experience with EI services. If her experiences with ECSE were less than satisfactory, I wondered about Emily's experience with the special education EI services that she received prior to the preschool program. She also reported negative experiences with the EI parent coaching she received. To Emily it felt like she was being "judged as a parent." She told me she stayed enrolled in EI to open more doors later. Emily described a manualized coaching model. The home visitor offered information, coached Emily on the strategies, and offered a critique of Emily's implementation.

EMILY: She would bring this packet every week, and she would be like this week we're going to work on this, this week we're going to work on this, and then every week she would try to train me. I was on the floor with her and him for an hour every Wednesday, and she would be like 'no, you're doing it wrong, you need to do it like this.' I was just like, 'I'm on the floor with my third child, okay. I don't know what to tell you'. At one point they were like, 'we're going to videotape [me] playing with him, and they critique it.'...It was just like, 'well we felt like you did this right, but you didn't... ' Anyway, Wednesdays were my day off of work, so I hadn't showered, I was in my pajamas...'

Emily had thought early intervention would be child-focused, that they'd come to the house and "play with your kid so you can do your own thing." But the interventionist 
said "no, this is for you." Emily remembers the intervention coach asking "How often do you get on the floor and play with your child." Emily said she told the interventionist "Not that often. I'm kind of busy." The interventionist told her she needed to be on the floor playing. To Emily this felt like "a guilt trip."

Like the other mothers, Emily felt like they needed the services to "open more doors." She said she "knew enough...to know that if I want to get him into a special preschool that I'm going to need to keep up with this." Karen told me "really I just wanted to bail on her."

Emily kept asking the EI person for a speech therapist and eventually one started coming to some of the home visits. Emily said it wasn't as helpful as the medical speech therapist. She also said "[the speech therapist] would use all this vocabulary that I didn't understand." Emily felt she understood some of the disconnect because "they have their standard they have to do."

EMILY: It's just funny because I know a lot of people [quit EI], and I know why. Because it's kind of offensive when they come in your house and they're like 'well, why aren't you on the floor playing with him for at least two hours a day'. I'm like, 'oh my gosh, if you lived my life.'

I engaged in repeated and in depth reflection on this portion of the transcript. I drew several conclusions from her comments. I had entered the research process aware of a bias I possessed towards educational early intervention programs for autism. I engaged in bracketing activities to address these biases and identify the lived-experience for Emily, independent of my own. In Chapter 5, I discuss the conclusions and themes with regard to EI for autism in more detail.

Support from parent groups and other social media resources. Emily told me 
that following Joshua's diagnosis she turned to social media. She said she listened to a podcast called "Autism Empowerment." She said "I started listening to that, and that was helpful. She described the ways in which other on-line groups were helpful:

EMILY: ...there is [also] Autism Support, an Autism Moms' Facebook page that's really, really big and I asked to join that, and that helped a lot... [because you can ask questions] and you can search it for all these past questions, so you can be like 'what ABA through this person or what services did you get through Kaiser [etc.] ...'

Ending our second interview. As our interview drew to a close I thanked Emily for talking with me and I explained the next steps in the process. By this time I had already been considering the possibility of a group member check meeting with all of the participants. I felt that the participants' stories were so valuable and I wanted to be sure I captured in the way that truly reflected the meaning each mom attributed to them. Emily expressed a strong interest over a group meeting and getting together with the other moms from the study.

\section{Narrative 3: Jessica's Lived Experiences ${ }^{3}$}

Meeting Jessica. Jessica and I met in her home a late morning in November. Jessica's husband was away at a medical conference. Both of her daughters were at home with her. Helen was the oldest and the child diagnosed with autism. She was upstairs during most of the interview. Jessica's two year old, Emma remained downstairs with us and engaged in a variety of developmentally appropriate activities that Jessica had set up in preparation for our interview.

Jessica's home was inviting and it felt like a home with small children. While

\footnotetext{
3 Jessica requested that all her photographs be omitted from the published dissertation in order to protect her family's privacy.
} 
everything was organized and neat Helen's drawings were framed and displayed. There were pictures of the children on the walls and toys, puzzles, and game available. Jessica offered me something to drink.

During the first interview, Jessica and I talked for about an hour and during this time Jessica responded politely to her daughters when they interrupted our conversation. She offered them gentle guidance and patience, attending their needs. I was impressed with Jessica's parenting. She was loving and respectful. I later found out that she was a trained special education teacher who had completely most of her work towards a masters degree. I could see she was experienced in child development and early childhood education. Jessica had immigrated to the United States and is fluent in English and her native language. Her husband, a medical doctor, was originally from another state.

Before we started the interview I explained the informed consent. After I reviewed the procedures for the first interview including reading the prompts out loud. Jessica spread the photographs out on the table. She had selected 17 pictures and lined them up chronologically. She told me she had spent a long time selecting the photographs and thinking of what she wanted to say.

I found Jessica's approach to sharing her story with me very interesting. Because it was chronological I had a new insight into the journey her family made from birth through diagnosis. I was already beginning to see themes emerge across the participants' lived experiences. My conversation with Jessica allowed me to organize this information as a 3-year journey into unchartered territory. While it may be said that all parents of infants and toddlers are on a journey, when I listened to Jessica I began to think that the 
journey parents of young children with autism is not well understood. In essence I wondered if they were blazing a trail without an experienced guide or an accurate map.

Jessica started our interview with a photograph from the hospital the day Helen was born. She picked up the photograph and smiled. In the photograph newborn Helen in in the hospital wearing a little newborn beanie. She is crying and turned towards the camera. The picture was taken by Jessica's husband moments after Helen's birth.

After a moment, Jessica told me it had been difficult to get pregnant and took nearly two years. She and her husband decided on fertility treatments and she got pregnant with Helen. The pregnancy was high risk because Helen had been one of twins and at 13 weeks gestation the other twin miscarried. She told me the whole experience was "an emotional rollercoaster."

Family life. Jessica and her husband feel that it is very important for the family to understand and support each other. She felt fortunate that her husband is understanding and they can talk about anything. They are on the same page and advocate for Helen. Early on her husband trusted her intuition and feelings about Helen's development even though "half of him wanted to disbelieve what was happening." For Jessica and her husband it was important to recognize that Helen is an individual and all they will do their best to help her become independent but she they also know she may not be able to live a completely independent life. They had a respect and understanding for how Helen relates to the world. They saw part of their job as parents as facilitators of Helen's development and engagement with the world. Perhaps more importantly she is a member of their "little family" and they have to understand and support each other. 
JESSICA: My husband and I always remind each other... no matter what it is, this is what we get. And you know she's individual...not our possession. So we're just going to do what is best for her as a parent...I think it's very important [that]...this little family understands each other and supports each other... I feel very fortunate that [my husband's] very understanding and you know we can talk about anything and we can come to the same page to advocate for her. So that's a very comforting...

Jessica said it was still a struggle sometimes when family members are in “disbelief” about Helen's autism diagnosis. They tell Jessica and her husband that Helen is "OK" and that as parents they are "too soft on [Helen]." I asked her why she thought this was their attitude. She attributed it partially to the generation gap. She also felt since they lived far away they didn't get to see Helen every day. This was another situation where Jessica and her husband both shared the same views about advocating for Helen. They felt the most important thing was for them to do what they "felt was right as parents." There was a family cohesion and unity that helped the extended family come to understand Helen's way of seeing the world.

JESSICA: when [extended family doubts Helen's autism] we always say 'what's important is us --we are the parent. they're not parents.' So we are the parents [and] need to be strong and really decide what's good for her. It doesn't really matter what everybody says. [My husband] always says "you can't buy the time back...if you think this is not right. You need to fix it right now. If you think this is the right thing to do for her you have to do it now.. And if you think you have to try, you have to try now."

Autism symptoms \& diagnoses. The second photograph Jessica showed me was a picture from Helen's second birthday party. It was at Gymboree, a preschool-age play gym. It was Helen's third birthday party. Six children stand in a circle as a teacher directs an activity clapping hands. In the picture Helen is wearing a crown and the parents stand back behind the children. 
JESSICA: [when the Gymboree teacher brings out bubbles]...Helen just shuts down and she sleeps. So I was thinking like hmmm this is not really like what is supposed to be they usually wake up - [that would be a more] appropriate reaction.

The picture had meaning to Jessica's lived experiences on several levels.

First, it represented the early days of the journey when it seemed Jessica was the only one who recognized that Helen had developmental issues. Jessica said, "I kind of knew from 6 months that Helen might be autistic." Jessica said she noticed "sound sensitivity" at places like Gymboree or mom's groups. A year earlier when Jessica and her husband went to visit family in on a tropical vacation, Helen had a similar sensory aversion to the sand on the beach. She said, "We took a picture and she was a year old and touching sand ... like the same sort of reaction." She looked over the photographs then pointed at one of the family on the beach. Helen was just under two-years-old. Jessica and her husband are looking at the camera smiling. Helen is looking down at her hands covered in sand.

When it came to seeking help from the pediatrician, Jessica described a similar experience as the first two participants. She recognized early markers of autism. That combined with her intuition that Helen's development was atypical, led her to seek help from her pediatrician. Although Jessica had concerns for Helen's development by 9 months she didn't bring it up to the pediatrician until 18 months. At that time the pediatrician did an M-CHAT. Helen scored borderline for risk for autism at that time. Jessica expressed her concerns but the doctor felt there might be a slight speech delay because English is Jessica's second language. Between 18 and 36 months when Jessica expressed concerns about autism, professionals told her (a) wait and see, (b) that the 
speech issues were related to the family's bilingualism, and (c) that the speech issues were a language delay and not autism. Jessica told me there were times when she unsure about trusting her own judgment over that of the professionals who were telling her Helen did not have autism.

JESSICA: But at the same time you know it felt like I'm not really [the] professional. I'm a mom so... if the a professional is saying to wait, maybe I should wait ...so I was kind of going back and forth, should I push more or should I just wait? And so it was hard but I was researching on my own, like reading the books.

Jessica did not give up trying to get help for Helen. Driven by her awareness of the importance of early intervention, Jessica finally insisted that the doctors assessed Hannah for autism when she turned three. Jessica told the doctor that ages three to five are a "critical time in development." She'd lost her patience waiting for a referral for a developmental evaluation. Her sentiment was "Let's move on and just get diagnosed." Helen was diagnosed with autism at 36 months.

Obstacles and challenges. I asked Jessica how it made her feel when the doctors and professionals wouldn't listen to her. Jessica told she would have felt more comfortable trusting her gut feeling if it had been "just one person that was saying 'don't worry." She explained:

JESSICA: ...If it was just one person ... saying 'don't worry about it,' and if everybody else was [saying] 'maybe something is there,' I would [have insisted], but what happened was, his parents, my husband, the pediatrician, ... all across the board, 'wait and see, wait and see.' So it [was] not just one person. ... I felt so little, like my knowledge and mother instinct, and all that...meant nothing basically. In looking back, I always feel what if I pushed more, what if I stood up more for her? It might have been different, but we can't take the time back.

Not only was it difficult for Jessica and her husband to get doctors to refer for an 
autism evaluation, but once they did agree to a referral to a developmental pediatrician, there was a 7-month lag between when Jessica requested a referral and the autism diagnosis. Jessica told me that in addition to getting the appropriate services for autism, a diagnosis opened doors to insurance coverage for services that would not otherwise be covered. All of the other participants reported the same understanding of the need for an autism diagnosis to access "a separate pot of money" for both educational services and medical services. In Jessica's case Helen had been receiving speech services through the medical side of things at 18 months. Helen had been diagnosed with a speech delay early on but the doctors and developmental specialists would not test for autism. As a result there was a problem with insurance and Jessica and her husband had to pay over $\$ 2,000$ and pull Helen out of speech therapy. The autism diagnosis would have covered speech and services would not have been interrupted.

JESSICA: They won't [pay for speech with a developmental delay diagnosis]. So initially when we started speech at the age of 18 months there was a miscommunication between the receptionist and insurance company and all that-they saw that there was some brain injury of her or something. But then of course there wasn't so we were told their insurance would cover. But then it came out, not...It was a lot...over $\$ 2000$.

RESEARCHER: Did you have to pay it?

JESSICA: Yeah...: I mean we were going to contest but then my husband is like "well nothing will change. We should just to pull her out immediately just to stop service and get the referral" and see what's [covered]. So when we checked with the insurance company they said that yes this speech is covered. right? but then the representative really explained what the exclusions were and the billing office at the private speech therapy. They didn't check it either. So they said it would be covered and it would be just co-payment of so and so..like other doctor's [visits]... So we took as what it is. And then the bill came and it said like denied insurance coverage. So we are like 'what's what do you mean denied?' They said 'oh there is an exclusion we assumed your daughter had some brain trauma or something but it seems like there is no you know code underneath when they were billing.'

Raising Helen in a neurotypical world. Jessica selected another picture to share 
and discuss. In the photograph Jessica's husband smiling into the camera. He has his arms around their two daughters. Helen is close to baby Emma and she is smiling and looking at the camera. They are in their family home and the girls are in pajamas. Jessica told me she included the picture because Helen rarely wants to be close to Emma and she "finally" got a family picture.

Introducing Helen to her baby sister. I asked Jessica to elaborate on the relationship between Helen and Emma. She said it was hard for Helen at first to understand having the baby around. As an infant Emma was unpredictable and that was hard for Helen. Jessica said "she was really scared in the beginning.... when I was in the hospital she never came close to me."

Jessica started laughing as she told me the strategy she used to help Helen deal with Emma and understand her baby sister. She had used their family cat as a comparison for Emma. We both found this very funny.

JESSICA: ... So [Helen] was staying in the corner of the room...with my mom who only speaks [another language]. So [it was] a huge change for her. And [Helen] was just staring [at her newborn sister] like what is this is like moving little thing? but --amazingly, she loves [our] cat... And so when we came home we [said] "don't worry this is just like another cat. She doesn't meow but it's ok." [Both laugh.]

JESSICA: And we said "[see] she has black hair. See the cat has black hair" and she's like "OK."

RESEARCHER: Oh I love it!

JESSICA: And then I just --it's almost like a cat meeting another cat. -- slowly, slowly, slowly getting into...another person's territory and [she's] like $O K$ well this one doesn't move quick. I guess I can handle it.

Sensory processing. Jessica told me about the accommodations she and her husband made in daily life to address Helen's sensory difficulties. They weren't simply to make Helen comfortable, they also functioned as supports for learning emotional 
regulation, social interaction, language, and engagement with everyday routines.

Helen's development is very impacted by sensory issues. Jessica described Helen's sensory processing as variable and fluctuating. So, accommodations must be flexible and responsive to Helen's experience.

JESSICA: ... the sensory thing, is not stable. It's changing all the time. So overall it's sensory but [unlike] some kids [where] it's always a sound...her thing is it changes. So when the sound sensitivity is better than texture becomes so much higher. And so it's a sensory thing is definitely impacting our life but sometimes it's ok. Other times we can't do the same thing that we [did] do a month ago.

Now Helen has more language skills and is able to communicate when something upsets her because of the sensory processing difficulties. However, early on it took some guesswork by Jessica and her husband. She shared an example. When Helen was younger they had to take down the vertical blinds behind the kitchen table. "If anybody touched it or it made a sound — the [heating] vent is underneath.” Jessica said Helen couldn't communicate so she would "just have a meltdown."

Jessica stopped, scanned the row of photographs and pointed at one of Helen when she was about three years old. She is clearly distressed, sitting on the floor crying. Helen is about four years old in the photograph and sitting on the kitchen floor. It is clear from the photograph that Helen is screaming and in distress. Jessica told me what a difference Helen's communication skills made to supporting Helen's sensory processing problems. She said:

JESSICA: [Back then] she shut down. But then these days... she can really explain to us [what's bothering her] so that's like super huge and easy... Like this vertical blind, [now] she could explain like "mom this is too much and I don't like the sound. Can you do something about it?" And we are like "Oh we better do something." 


\section{Supporting Helen in typical childhood activities.}

Helen's fourth birthday. During our interview Jessica used the photographs to show me occasions when Helen experienced success in everyday situations. Jessica shared photographs and stories about Helen's fourth birthday party, swim lessons, a family trip to the tulip farm, and preschool. Jessica selected picture number J-7 from Helen's fourth birthday party.

JESSICA:....we were like well it's her birthday we want her to be happy but in order to do that, [we had to figure out] how can we make her birthday more accommodating to her and in meeting her needs.

The year before at Gymboree wasn't successful because of Helen's sensory issues and difficulty following along. Helen ended up crying and having a meltdown. Jessica shared with me how hard it was and how much she wanted Helen to feel special because it was her special day. For Helen's fourth birthday she decided to help set up the party for success beforehand. Jessica and Helen practiced going through the mall, back and forth in the morning. The party was a Build a Bear, a toy store in the mall where children stuff their own teddy bears. Jessica went to the store beforehand and told them about accommodations Helen would need.

JESSICA: We really explained to them [that] Helen... has some sensitivities so please don't push. [Don't put sound makers in the toys] ...it's already too much stimulation to be in the mall.

Jessica told me the party went really well. The employees led the group as they usually would but because it ran smoothly because Jessica had explained that Helen "probably won't be able to follow along so don't worry about it, just carry on." She told them "just do things she seems to be interested in." She said they were able to do that. 
The party was successful. Jessica shared her feelings about making accommodations so Helen can participate in typical childhood activities:

JESSICA: [we] just want her to have as much experience as possible like neurotypical kids even though [there's some] things we can't provide but we want to try. I mean, if it fails like oh well. -- Just have to move on...

Picture J-7 was taken at Helen's fourth birthday party in the Build A Bear Store. Helen is holding a stuffed dinosaur and wearing a crown. Her friend is hugging her and Helen is smiling. The store and other children can be seen in the background. Jessica told me it made her feel good as a mom to see her daughter happy, smiling, and hugging her friend.

JESSICA: ...it's her best friend and then...we see that smile [to picture] and we are just like...'you know, you can't beat that.'

Jessica looked down at the picture and smiled. She looked at me again. I sensed it meant a lot to her to have been able to make Helen's birthday special. I considered how that is the goal of most parents to see their children confident and happy. I later reflected on this in my research journal; this aim was not so different for the participants in my study. For parents of children with autism parenting requires special effort, ingenuity, and an ability to advocate in order to make the neurotypical world accessible.

Swimming Lessons. We returned to the photographs and Jessica glanced over them trying to decide which one to choose. I suggested we go back to the prompts and select one to get started. I read two prompts "select a picture you remember well. Tell me about that day and what your family's doing" and "Select a photograph and tell me why you brought it to talk about." Jessica decided on a photograph of Helen in a pool, back floating with the assistance of a swim instructor who was standing behind Helen, 
supporting her as she floated. Helen's body looks relaxed and the pool appears empty except for Helen and her instructor. All of Jessica's photographs, including this one, looked like pictures of any young child participating in recreational activities.

When Jessica explained to me what went into enrolling Helen in a program where she would be successful, I was very surprised and I calculated what goes into navigating recreation programs, schools, services, and daily activities. Jessica and her husband "want to give her the opportunities but," Jessica said "there's a limitation to what we can provide." It's not as simple as having a 1:1 assistant that the community center typically provides. First of all community center assistants change all the time and Helen would not do well with that kind of inconsistency. Jessica also told me "as parents we had concerns like water safety.” They didn’t want to risk Helen's safety.

Helen really wanted to do swim classes because all the other kids were doing it. The kids talked about it all summer long. So Jessica and her husband said "ok we can try." So she researched a lot and luckily her recreation district had an adaptive aquatic class. I asked Jessica what would have happened if she hadn't found an adaptive class for Helen. She explained:

JESSICA: You really have to look into private lessons and then really pay significant amount to provide something like remotely close to what the other kids her age are doing... they actually ask a questionnaire like 'what kind of things trigger her and how can we help with that?' And so they've been very accommodating.

RESEARCHER: And she's happy? She likes it?

JESSICA: She is super happy, she just jumps in.

Interview 2: A deeper look at services.

Piecemealing a specialized autism program for Helen. Like the other parents in 
this study, Jessica became effective at navigating three major service systems for early autism (i.e., medical, educational, and county). In Jessica's case Helen did not receive autism services until 4 years old. She received a medical diagnosis at 36 months. She was diagnosed with a language delay before 36 months but had to stop speech therapy because the insurance made a mistake and Helen was ineligible. Helen was not eligible for county services because her disability was not deemed significant enough. She also was denied speech services from the educational program. Helen and her husband took an accepting attitude about the state of service systems for autism. Their goals remained on Helen's development and access to typical childhood experiences. In total Helen's current services total just under 20 hours. This level of service is consistent with recommended practices for young children with autism (Zwaigenbaum et al., 2015). Of those 20 autism-related service hours per week, Jessica and her husband pay for 16 . The early childhood special education program pays for 4.25 hours.

I asked Jessica to tell me more about the ECSE services and whether she was happy with them. She said that the special education preschool has a one to three student: teacher ratio. They work with the children on early skills and activities like circle time.

JESSICA: ...The preschool is three hours, four times a week, and then the tutor we have every week for two hours.

RESEARCHER: And then she goes to the Special Ed preschool?

JESSICA: Mm-hmm, on top of that, two hours, yes.

RESEARCHER: So the District IFSP you get four hours, and then the once a month consultant?

JESSICA: That's it, yes. Everything else is our own private.

RESEARCHER: Okay. Does she get speech too?

JESSICA: Yes, we do speech and OT.

RESEARCHER: And you pay for it, or your insurance? 
JESSICA: Yes [we pay for it privately].

Jessica and her husband felt that ABA would have been good for Helen but they couldn't afford it with all the other services. Jessica and her husband built a service plan for Helen based on "areas of service [Helen] needs and what they can afford." Originally they looked into ABA but buying an insurance plan that covered it or paying out of pocket wouldn't work. Jessica told me that the education service district doesn't offer ABA services.

Our conversation turned back to the early intervention services Helen received before she turned 3. She had received limited services for a speech delay and not autism. Jessica told me that an early intervention specialist came to their house once a month and coached Jessica. Then Jessica told me something that echoed the sentiments of the other mothers, "It is basically [to get] your name in the system. That's what it is."

Services through the educational system. Given the problem with insurance coverage I asked Jessica about the educational services Helen received before and after the autism diagnosis and if there was a way to get the speech services through the educational service district. Jessica said they didn't offer that to them because Helen did not have an autism diagnosis. She only had a developmental delay educational eligibility. Jessica said early intervention home visits were the only service offered to her family before Helen turned 3. An early interventionist visited Jessica's home once a month and conducted parent coaching. There was no speech therapy. Jessica told me that the interventionist would do coaching, get on the floor and play. During these home visits Jessica tried to point out Helen's autistic behaviors to the interventionist but again she 
was met with invalidation.

JESSICA: ... I'd been telling her, 'look this is a very autistic behavior like lining things up. '...[The interventionist's] take was like 'well my son was like this and da da da.' Anecdotal stories... I'm like 'OK well [that] might help [your son] but it really doesn't help my daughter.'

RESEARCHER: So did she give you strategies to or not anything? I mean clearly ... you're a great mom. You know how to manage everything with all kinds of activities. Does she do anything like that? Or does she help?

JESSICA: She [was] usually like, 'Oh it seems like you're doing this activity already.' Basically that kind of stuff...So it --I would say it's a meaningless but then depending on what your goal is [maybe some parents find it beneficial].

When Helen turned three, she received early childhood special education services.

Overall Jessica did not feel the early childhood educational services (i.e., ages three to five) were sufficient nor particularly beneficial to Helen's development. However, like the other parents she expressed that she saw enrollment in EI and ECSE as a way to get special education services in place so the transition to kindergarten would be easier. She told me that the educational services are "very minimum" and they "don't rely on it." She said that she and her husband's main focus is so she can have "a smooth transition to kindergarten public school."

Summary: A new way of life. During our second interview Jessica made the connection between all the supports she has put in place for Helen and how far Helen has come. She shared with me that recently Helen told her that the toilet flushing hurt her ears. It was hard for Helen to be in the bathroom because of the flushing sound. When Helen told Jessica and her husband they put in a quieter mechanism and it solved the problem. I considered the positive impact of Jessica and her husband scaffolding Helen's emerging self-advocacy and ability to recognize sensory triggers.

JESSICA: We changed the toilet too. It was the flushing sound, it's like a 
sensory trigger every time. So we changed it to quiet fill to see if it works, and it worked, and she said, "thank you, Mommy, this works fine."

Jessica said it wasn't until she looked at the photographs and talked about them with me that she realized how far Helen has come and how much her and her husband's support has helped their daughter.

Jessica shared a picture of Helen at preschool. The picture depicts Helen in her classroom during circle time. About ten preschool ae children sit in a circle while Helen stands in front of her teacher. She is wearing a special crown and the class is celebrating her birthday. It represented the ways the school and Jessica accommodated for some of the challenges autism created in Helen's life. In this photograph Helen is at school. She was just turning three and every so often the class had a special routine for kids with birthdays every month. Because Helen's birthday is in December she would have a "meltdown" every time the other kids got to wear a crown and have a special day. The photograph depicted Helen's turn for a special routine. Jessica explained this photograph had significance because it represented how far Helen had come with help and flexibility from her teachers and through her own support. Now these kinds of activities do not trigger tantrums. Jessica helped bridge the autistic and the neurotypical worlds. Now she and Helen read "social stories" before these kinds of activities and through experience at the preschool, Helen understands routines such as these.

The first half of the photographs she shared with me depicted the early symptoms of undiagnosed autism. The later photographs included descriptions of the supports that helped Helen experience success in her daily life. I found Jessica's stories very touching. I thought about what a gift it is for a parent to make a neurotypical world work for her 
child with autism. I journaled about this quite a bit after my interview with Jessica. I spent some time reflecting on the world as it must be to a child with autism. I thought of Caroline's view of the differences in the "autistic brain" and how people with autism are relating to the world the way it occurs to them. I began to see that Jessica while sharing a unique lived experience shared a number of experiences in common with the other participants.

\section{Narrative 4: Karen's Lived Experiences}

Meeting Karen. I had hoped to recruit three to five participants. After the screening process, I had three participants and settled on three for my study. I had already begun interviews with the participants when I received an email from Karen.

Hi Donna, My name is Karen M. My son, Carter is four years old. He is impacted by non-verbal Autism, diagnosed at age 2 . Caroline T. encouraged me to contact you regarding your study. Please let me know how I may be of help. Thanks, Karen

I wrote Karen back and conducted the phone screening to confirm she met the study's inclusion criteria and that she remained interested in the study. She wanted to participate. Like the other participants I felt Karen was eager to help with my research and that the goal of giving voice to parents of young children with autism was important to her.

Karen and I met in a conference room available to residents in her apartment building. She was immediately friendly and open. I set the prompts on the table and asked her to place the photographs in front of us In any order. She decided to talk about the photographs chronologically. I found out later that the first five photographs represented the first part of her journey to diagnosis and treatment for her son Carter. The 
second five photographs marked a positive transition in their family life and settling into a new way of life. Unlike the other participants, Karen and I met for three interview sessions, each one approximately 1.5 hours. This unexpected additional meeting was consistent with phenomenological methodology (Vagle, 2014) and I took measures every step of the way to insure that Karen knew she was in control of the amount of time and number of meetings. Our total interview time was nearly 5 hours compared to approximately 3 hours for each of the other participants.

From a practical perspective the amount of interview time was necessary. Her journey to autism diagnosis and services had a very difficult beginning. Karen had had a hard pregnancy and postpartum period, suffering from depression through both. Karen described the experiences during the birth process as "traumatic." She had to have an emergency C-Section and had a serious infection and complications. To make matters worse Carter had complex developmental and health issues throughout his first three years. He had and continues to have difficulty sleeping. Karen reported that she rarely has gotten more than 2-3 hours of uninterrupted sleep since Carter was born.

Karen, her husband, and Carter live in a well-appointed supported housing apartment building. Karen stays at home managing Carter's programs and coordinating services. Her husband works part time to full time. Karen told me she has completed two graduate degree programs and was a licensed kindergarten teacher for many years before becoming pregnant with Carter.

\section{Family life.}

Starting the journey as a family. Karen's photographs were printed on quality 
photographic printer paper. She presented them chronologically and she laid them out on the table. The first photograph (K-1, Figure 14) was a picture of Karen, her husband, and Carter. Karen told me Carter was only 3-4 weeks old in the picture. Karen's husband Mike was carrying him in a Baby Bjorn. Karen and Mike were smiling in the photograph. I would find out later that Karen's smile in the first several photographs was somewhat inauthentic. She described herself in the photographs as "in denial," "the face of postpartum depression.”

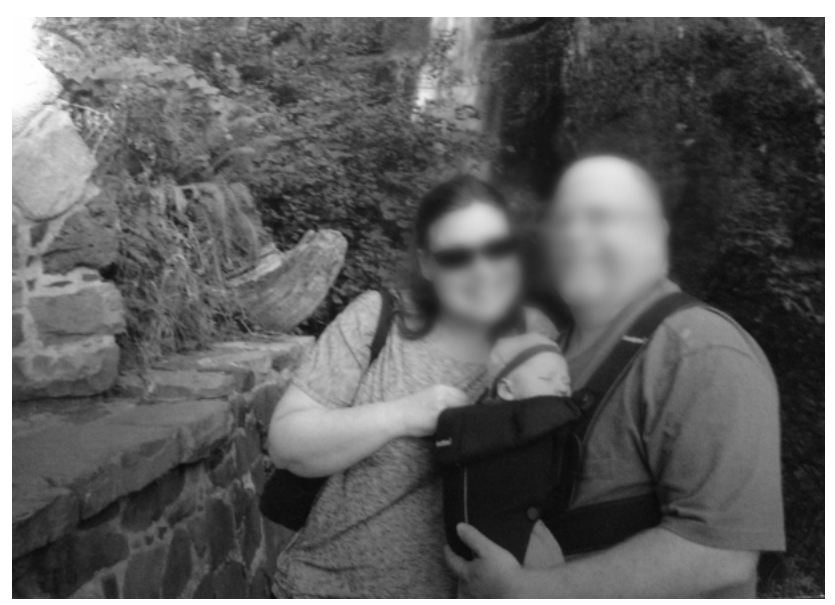

Figure 14. Photograph K-1 depicts Karen, her husband, and Carter on a family hike. The photograph was taken four weeks after a C-section and Carter's delivery. Karen climbed to the top of a waterfall trail. She describes herself in this photograph as being "in denial."

During those early days of motherhood, Karen was exhausted, depressed, and without support. Part of the isolation was because Karen and Mike had told family not to come visit until a month or two after the baby arrived. They had planned a homebirth and to take three weeks together, not leave the house and just bond with the baby.

Karen expressed that the photograph was significant to her because she it represented “... total denial... like if I could just do life how I thought it was going to be..." It was a physical feat to climb to the top of the waterfalls at a historical site an hour 
from home with her month old infant. Karen was still recovering from a C-section. I looked at the photograph for a long time. I agreed with her, it looked like a picture of a young, happy family. I couldn't help but think of her physical, emotional, and psychological strength and resilience.

KAREN: That was our first time the out of the house . And I hiked up [local water fall]. I was probably just bleeding at that time like I mean it was bad...in that picture is I'm smiling and everything looks great. And like inside. I'm like...it's so, so hard...when I look at this picture, back at the day I posted this on Facebook a while later...it was a total denial.

Karen and I talked for over 30 minutes about her pregnancy and complications.

We sat at the small round table in the empty recreation room in her apartment building. It was a typical Pacific Northwest day, raining and cloudy. As she told me about those early days both looked down at photograph S-1 often. To me it represented the start of Karen's family's journey.

Family characteristics, belonging, and likeness.

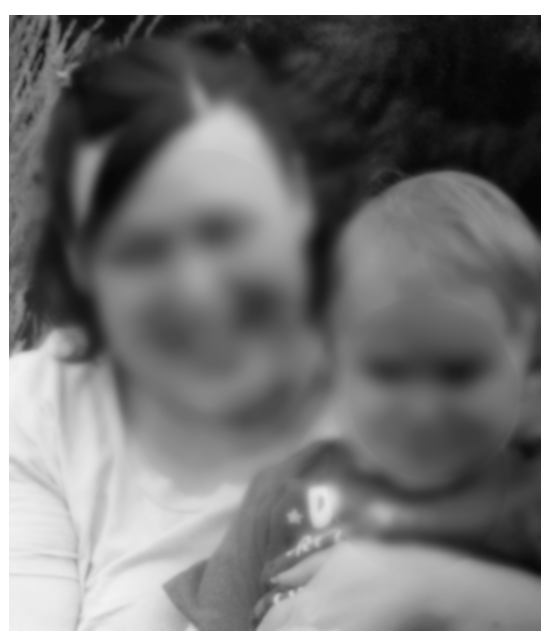

Figure 15. Photograph K-2 was taken on Carter's $3^{\text {rd }}$ birthday. Karen loves this picture because she and Carter have the same expression of disdain for the older children misbehaving in a toddler play area. She fondly calls Carter and herself in the picture "the judgment twins." 
Photograph K-2 (Figure 15) marked the end of some of the psychological challenges in parenting Carter. It marked the point where she started receiving support and finding answers. The photograph was taken at the children's museum, a noisy and busy environment that is always difficult for Carter. The picture was taken right after he received his formal autism diagnosis. It also had meaning to Karen because she and Carter shared an experience and were aligned. They'd gone to the museum for his birthday. They were in the toddler garden when some older children came into the area where they were not supposed to be. They were rough housing and ruining the fun for the toddlers. Karen loves this picture because she could see herself in her son. He'd lost all language but his feelings about the situation were clear, and Karen recognized them; they were her own feelings. To me this story represented the bond between Karen and Carter. It also represented his belonging to her and to their family.

KAREN: My sister in law was up here visiting because they come up for every birthday... And my husband was there and I think grandma was there. We're [at] the children's museum... The reason I love this picture--it's not even the best picture -it's that with the C-Section and everything that happened it seems really crazy, but for the longest time I wasn't even sure he was my child...And then I had a very difficult baby we had like bonding issues...My sister in law snapped it...You had asked about parenting about ...[photographs] that remind you of your parenting. We were in the toddler garden of the museum's outdoor space ....and there's little kids running around. ...He had just turned three and he was still kind of toddling because he had kind of lost his balance.. in the regression. He had a hard time wearing shoes. They bothered his feet...[He was] really sensitive...some older kids --like 10 year olds... showed up in the toddler garden and were acting like jerks...splashing the little kids and throwing dirt... And so I totally call this photo judgment twins because--

RESEARCHER: You are both are alike?

KAREN: Oh yes we are both looking at that and...we look like [demonstrates how looking] 'THAT is not how you behave in the toddler garden! Like really?' ...It was not a planned picture. Super candid [my sister in law] was just clicking, clicking, clicking. We have somewhere we're looking and kind of smiling you know but this one is judgment twins....And the reason I like it to you is because 
that was still during his silent period...He hadn't even started vocal stimming yet-he has a very strong vocal stim where he hums. So he hums[demonstrates humming] ]but he was even doing that yet...he just was so silent. And [looking at the picture] I was like he has an opinion I'm like. he has an opinion and it matches mine. Like he doesn't have to talk to be expressive because he is very annoyed in that photo as am I... That's what that picture is about to me.

\section{Early autism symptoms and diagnosis.}

Karen's early concerns for Carter's development. Despite Carter's early sleep issues and gastrointestinal reflux, Karen also had concerns for his development. Carter had regressive autism and at 18 months lost all of his emerging language and gesturing after a short illness. However, Karen had concerns much earlier and tracked Carter's development through the on-line Ages \& Stages Questionnaire (ASQ; Bricker, 1999). She was driven by her intuition that his development was delayed. However, while Carter lagged in early milestones, he always caught up. "He was meeting things late and it was getting later and later." She didn't necessarily think it was autism until after he regressed at 18 months, but from early on she thought he had a "physical problem." As soon as she got worried, she told me "I'd be assured by other people that there's nothing wrong with this kid. Like 'look he's looking at me and he's playing with me.”'

KAREN: And I was like wow... more kids [are] becoming autistic and... I don't know maybe [it was] like a worst fear. So it wasn't necessarily [that] I suspected he had autism... I just thought he had this physical problem.

Because Carter had regressive autism he was still engaged socially early on.

Karen described him as a social baby. His language was developing. However, When Carter was 9 months old she brought him to a new pediatrician about his GERD. During the visit the doctor referred Karen to early intervention services based on her observations of Carter's development. Even though Karen herself had a persistent 
concern about Carter's and his scores on the ASQ, she did not agree with the doctor. She did not think Carter needed EI.. In fact she became emphatic about it, angry.

KAREN: I was just super offended [by the new pediatrician who referred to EI]. We never did go back that doctor. I... felt blindsided. I was just 'oh yeah there's nothing wrong with my baby.' She [said] 'Don't worry. Just go to early intervention.' ...I don't remember a lot of that appointment. Yeah I heard her saying 'just go to early intervention...they get kids turned around If you get it done early now ... you won't even notice any differences in a few months.'... early intervention called a few times and they finally called [and said] 'If you don't call back, we're not going to call back -' and I called back [and said] 'No'-I left a message I'm like 'we don't agree with that doctor. We're going to let [Carter] develop on his own schedule...if we're concerned we'll call you back...'

Karen sought a second opinion from Carter's regular pediatrician and was

reassured.

KAREN: I was calling my doctor... [and said] 'they're saying there's something wrong with Carter' and [she's] like 'Why?... what did [the GERD doctor] see that I don't' see?' [I told her] '[the doctor] said he's not talking, he's not babbling about certain objects and he's not sitting up.. and he's not crawling yet.' --[The regular pediatrician said] 'I don't think that... that's really early....some of the things you're mentioning are actually a 12 month milestone, not a nine month. There's nothing wrong with you baby. You don't have to worry about it.'

After the referral to early intervention Karen stopped taking the ASQ's online and tried not to worry about Carter's development. She sought and received reassurance from professionals, family, and friends. She did not seek a developmental evaluation for Carter until after his dramatic developmental regression at 18 months. Carter remained a social, bubbly baby. He continued to have sleep issues and that affected Karen's physical and mental health. She said "I stopped looking for pathology in my child."

\section{Carter's early manifestations of autism.}

Sensory sensitivities. Prior to the regression, Karen had already started noticing differences in the way Carter experienced the world. Sensory processing issues were 
among the first areas that Karen noticed. As she explained his aversion for certain sensory experiences, she picked up photograph K-3 (Figure 16).

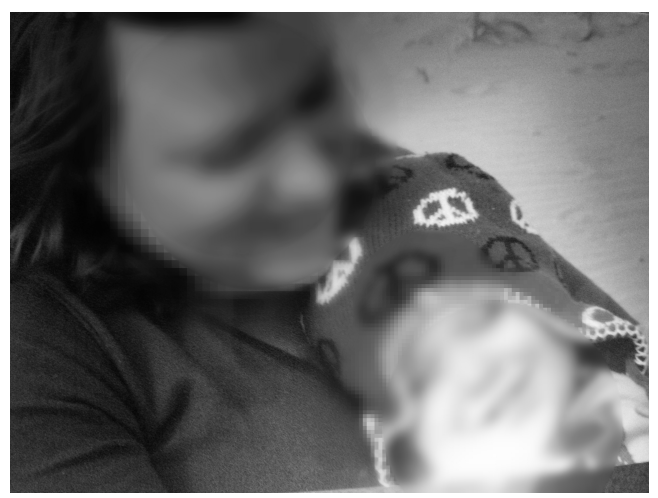

Figure 16. Photograph K-3. A family beach day. Karen remembers Carter's strong aversion to sand.

She looked at the photograph a moment then placed it in front of me. She kept her eyes on the photograph most of the time she spoke. She told me about that day at the beach. It was the last day before the sickness [that preceded the regression]. Carter was almost 20 months old in the picture. Karen remembered he started screaming when she put him in the sand. "He would be just like clinging to me like that because he didn't want anything to do with the sand." He stood there screaming and froze. He couldn't tolerate it. It was the same with walking in the grass. Karen said he was probably having symptoms of autism before the regression and they didn't know it was autism. She said he would "only walk on the carpet. He wouldn't walk on cement or grass." Karen said "this picture is actually about sand." That day in the sand was "the last day [Carter] was well." After that he became ill with a common virus and after he recovered physically, he experienced a dramatic developmental regression.

Social communication. Karen told me that before the regression Carter liked playing 
baby games and would laugh a lot during patty cake or peek-a-boo. He did not exhibit early delays in social communication.

KAREN:...he would want to play with us ... lots. We even did patty cake games and ... peek a boo games ...

RESEARCHER: Or like throwing the menu on the ground and make you pick it up?

KAREN: It was always a spoon for him. The spoon. The spoon. Or the bottle. Get bottle. Bottle. Bottle. so it was always .... lot of that. [After he regressed] we would be like 'Carter, Carter' try to get in his face [to get his attention]... Yeah and he avoided it. avoided it. avoided it.

Language. We had been talking for almost an hour. I asked Karen if she wanted a break. I realized there was a lot more to her story so I offered to stop and offered to pick up where we left off when we met again for our second interview. Karen said she had time because Carter was at his ABA class. She wanted to keep talking if I had the time. I was available and interested in hearing more so we continued. I asked Karen about Carter's language development before his developmental regression at 18 months. She told me that he had been somewhat delayed but then always caught up. Karen had been proud of his consonant sounds. She loved and she "always loved listening to his voice." "Da Da" was his first word. A while later he said "kitty." She joked "mama" was the last thing. He had started to say "mama" not long before the regression.

KAREN:... he didn't talk a lot before...he had like ma ma, da da, ba ba, kitty, $b a b y$. it was pretty much like that... He vocalized a lot... just a lot of exploring sounds. I remember thinking I was so impressed because he could make so many consonant sounds. They were in isolation but, you know he could $[t][t]$. He would sit there and play $[t][t] t[t][t],[d][d] d[d]$. You know? That kind of stuff.

Carter's developmental regression. Karen showed me another photograph. She said she included the photograph because it was the first photograph after Carter regressed and lost language, eye contact, and social skills. Photographs K-4 and K-5 (Figure 17) 
compares Carter's engagement before and after he regressed.

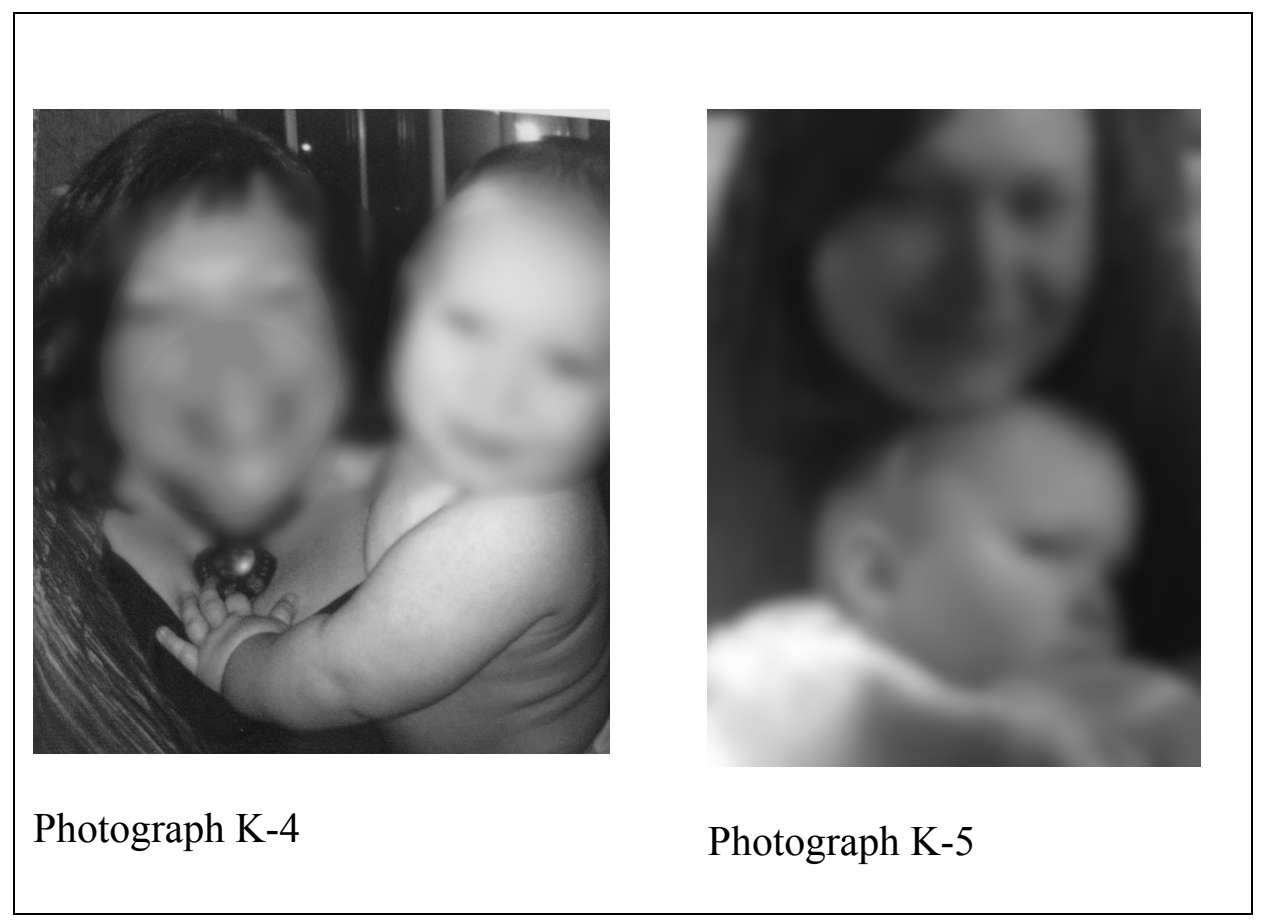

Figure 17. Box display of comparison between photographs K-4 and K-5. Photographs of Karen and Carter at 12 months and again at 20 months. The second photograph is immediately following his developmental regression. Karen described carter as "regressing inside himself." It would be a year before he smiled again.

KAREN: ... after the sickness he didn't look at us anymore. He didn't smile...that's what this I think this picture (i.e., ) is all about...he's already looking off into nowhere... He would just kind of stare off. He really regressed inside himself, really isolated himself. He's letting me hold him and snuggle him [at 20 months in picture S-5] because he needs the comfort and he's sick... but for the longest time after that we couldn't hug him, couldn't give him kisses... we couldn't touch him very much [except] to change [his diaper]...He would scream it was like touch was too much. Everything was too much after that sickness, the entire world like bombarded him. ... [that was] when everything started happening...we both got [a virus]- I had gone to Kaiser ...[because]... I remember afterwards --after we got home-- being like Oh my gosh he followed me into the bathroom at Kaiser... I didn't wash his hands and saw him touch the tile. I was really germ phobic still with him and I was like Oh we're probably going to get sick and we both did totally get sick and he ended up with a really bad virus... He was sick for almost two weeks and we were going to the doctor every day and they didn't know what was wrong with him and he had a high fever off and on. And we ended up having to take him to a hospital get rehydrated. They had to catheterize him and I remember the entire experience was so traumatic... and he was screaming and that was the last time I heard him say 'mama.' It was in the hospital screaming 
'Momma. Momma!'...After that sickness he was different...after that that I did the ASQ and I was like he can't do any of those things...To me that was the turning point because it was after he got better [from the virus]... I realized I hadn't [heard him] say anything in a while and he was starting to get into...staring at electronics and he...had a far away stare. He would just look through you and not look at you and he didn't respond to his name anymore...he starting to walk on his tippy toes. He's starting to flap his hands...I think it was the walking on the tippy toes...he had [never] done that. He started walking at 13 months. And this was 20 months already and he had walked normally that whole time...

Carter's loss of skills was dramatic. Karen and her husband weren't the only ones who noticed it. As we looked at Photograph S-5, our conversation turned to a memory Karen had around the time the photograph was taken. She said she remembered that her mom had sent Carter a wagon for his second birthday. She took a picture of Carter in the wagon and emailed it to her dad to thank them for the gift. Her father emailed her back right away. She recalled his reaction to seeing Carter in the photos. Her dad asked "What's going on with Carter in these pictures? Is he sick in these pictures? Is he scared or sad?" Karen said she broke down and cried. She told him "No dad...That's just how he is now. Nobody knows what's wrong with him. I can't get any help...for 11 months.”

When Carter was about 30 months Karen and her husband took Carter to a developmental pediatrician with their concern that Carter had autism. She told me the doctor made a preliminary diagnosis. The doctor had done an autism screening and told Karen and her husband that Carter's behaviors and score was "consistent with an autism diagnosis." Karen remembers the doctor was hesitant to use the "autism label." She wasn't sure if he was being cautious to help them adjust to the possibility of Carter having autism but something in his delivery of the preliminary diagnosis captured Karen's attention. She said she wasn't sure if he thought they "looked shocked" but 
Karen remembers telling the doctor they agreed. They saw the same developmental issues. The autism diagnosis would not be confirmed until after a standardized assessment was completed. The doctor asked Karen if she had any questions and then gave her "a lot of paperwork."

Waiting list for autism evaluation. While Karen reported relief in the preliminary autism diagnosis, she immediately encountered a major obstacle to getting help for Carter. After the diagnosis they decided to schedule another appointment with the developmental pediatrician to do the ADOS. When Karen called to make the appointment the receptionist told her that there was a one-year wait list.

RESEARCHER: Wait a minute they suspected autism and the wait was another year?

KAREN: Basically the one-hour appointment with [the developmental pediatrician]...was the developmental screening and [finding out] where do we go from there...his [doctor said] 'I suspect autism. We're going to need to do the $A D O S$ ' and his wait for that was another year ... So that was January...[Another option was] I could wait until July and...we could go see the developmental psychologist who could also give the ADOS --Those are the only two people in all of [their Health Care Provider's Network] ... qualified to do the ADOS...

Navigating service systems. Karen's initiation into service navigation for Carter had begun earlier with his health issues, but the difficulty getting the diagnosis was the first experience with the autism service system. Other participants as well as Karen all felt that one of the greatest difficulties as a parent of a child with autism is navigating the three major service systems: educational, medical, and county. Karen, like the other parents started with the medical system.

Changes in services. Karen told me that services for young children with autism can unexpectedly change or get cut off. There is a problem with consistency of professionals 
and continuity of services. This is inconvenient. It also has implications for treatment

consistency and success.

KAREN: When service years change you get cut off. You have already planned your life around the services. Like you used to have services at Providence every Friday at 11 . The year ended and you lost the spot. When you got it approved again the therapist was scheduling out six months and she doesn't have any solid recurring date so can you come one time on one week and another on another week. That happens every year...You lose that spot and then they say we need to get it approved, and that takes two months. Okay, that takes two months. 'Great, we got your approval. Okay, well it looks like your therapist is scheduling out six months from now. So then, she doesn't really have anything solid for you so you can come Tuesday the 17th at 2, and then the following Thursday at 4, and then...' and how do you live your life like that?...[That's] every year.

The connection between financial resources and Carter's development is direct and

bidirectional. Carter's autism impacts family income and opportunity.

KAREN:...We have a two bedroom now for $\$ 720$, because this is controlled through tax credits. Almost everybody here has a story. There's hardly anybody here that's like, "I'm in generational poverty. This is just my lifestyle," or whatever. People became disabled or whatever. I have a disabled son. This is how it went. If we still had to make that rent or whatever, we would not make these choices. He would be... I can't say institutionalized, because that's not how we do it any more... But he wouldn't be in the care of others constantly, and it would not be like this.

RESEARCHER: He would be forced into doing things he doesn't want to do. KAREN: And not making the progress. Who was going to say...

RESEARCHER: Who is going to say he actually does have language?

KAREN: He can spell; he does have this; he does have that. He has huge language. He's typing in sentences or whatever, words that... I taught kindergarten, and none of those kids could spell.

RESEARCHER: Right. Even with all this sleep deprivation stuff, you're seeing the shift to symbolic language.

Social media, a vital source of help. The value of social media and internet

resources came up repeatedly during our three interviews. Much of the support came in the form of encouragement and validation Karen reported that autism Facebook groups are places where people understand. 
KAREN:... to be able to go when you've had a very particular day and you're feeling like I'm failing my kid. To hear people [say], 'I felt like that too, but it's not that way.' And to get that support. Or just to go in and be like, 'my kid walked up to the potty today and lifted the lid up. He didn't sit on it.' It's such a big deal. 'Oh my gosh, my kid that will only eats crackers ate a piece of cheese' ....and they're like 'Oh my goodness!' If you were to post that on your [non autism group] Facebook, 'Carter ate a piece of cheese' they'd be like so what? Nobody gets it. But you totally go in there and you're like 'yeah my kid didn't bang his head today' or just something so specific to autism, where they're just like 'oh yeah. I had a night like this...I remember those days.'

\section{Obstacles and challenges.}

Postpartum depression, isolation, and a high needs baby. Carter's infancy was a

difficult time for the family. Carter cried inconsolably and slept very little. Karen was unable to work because Carter required constant attention day and night. That left Karen's husband to support the family so he wasn't available to help much with Carter. Her family lived far away. She was isolated and desperate for sleep. Most nights she didn't get more than 2 hours of sleep. Karen described the only way she could get a break:

KAREN: I got a little break he wouldn't be crying. He wouldn't be super needy. He could be in his car seat because the other thing is he could never to be put down-like we had hold him. hold him, hold him. hold him. or wear him up right. Always upright. Because of the GERD or... you'd put him down and he'd scream and cry, but he could be in his car seat and the road vibration would put him to sleep. He's just so super sensitive and so super perceptive. If we would stop at a red light -wake up. I mean we can stop for five seconds in the car. So that's why I'd go [and drive] 20 miles an hour on [local freeway loop] but we're moving as long as we're moving the car is vibrating, he's going to sleep. So it was like that. So like I wouldn't get a nap or whatever but he would sleep. And I would get a break and I would be like I have snacks or whatever in the car.

Carter's needs took a toll on Karen's life. She hardly ate at all, subsisting on coffee.

She was sleep deprived and exhausted. It was hard to get anything done because if she wasn't holding Carter he was screaming. She told me that sometimes she would put 
Carter in the baby carrier and go for a walk. She'd go to a grocery outlet just to be in the presence of other people. She was so alone and isolated and depressed that she felt like she couldn't connect with others because she would break down. Sometimes she felt like she'd lost her maternal connection to Carter. However, her love for her son never waned and she shared with me many times during our interview when she would come back to her connection to him, how much she loved him. Despite the relentless difficulties and lack of support, their bond was strong.

KAREN: [We would] go for a walk around the apartment community [or] WINCO...even if we didn't buy anything, [we'd] go for a walk to WINCO. Let's do this because it would be outside and walking with fresh air. Even just seeing people in the street. I didn't feel so isolated.

Karen told me that sleep was the biggest issue. If there was one thing she could change it would be the sleep deprivation. I thought it was powerful that she didn't say she would change Carter's autism but the sleep issues.

KAREN: I say [it] all the time... if I could change one thing --if I could change what autism means for us --for one thing it be...just the sleep. That's it. [When we get sleep] he's better. We're better. Life is better when we sleep.

Physical toll of Carter's sleep issues. At our third interview Karen told me that the night before Carter was up at 2:00 a.m. She described her physical health lately:

KAREN: He finally woke me up [at 3:00 a.m.). I had a migraine yesterday. it was so bad because it's been so many nights in a row. I had a migraine yesterday so bad I ended up vomiting at his OT appointment. I could barely drive us home and I'm like "tonight I've got to get some sleep.... and he did it again."

Karen's relationship with other parents in autism groups has been lasting. Recently, Carter's sleep issues worsened and Karen reached out to the parents on Facebook at 4:00 a.m. 
KAREN: I'm just desperate. I' $m$ on the phone at 4:00 in the morning out in the living room, in tears. My husband is in there with him because I had to get out of bed...I haven't had sleep in days...I was on Facebook this morning at 4:30 AM on my Autism Mom's group. Basically, I'm in tears, on it reaching out for a lifeline going like, "I need permission to get my kid on sleep meds." Because we've done everything our doctor has wanted us to do. It's not that she said he won't medicate him. I don't want my child medicated...

Limited financial resources. Karen and her husband's experiences with navigating services was complicated by their limited financial resources. As a result of Carter's complex care and service coordination needs, Karen was needed at home to care for carter and manage services. She was unable to finish her graduate degree. Prior to beginning graduate school she had been a Kindergarten teacher. Attending graduate school was a way of expanding her career opportunities. Karen had completed all the coursework and a culminating project for a master's degree in sociology. Karen and her husband now live at the poverty line so she can manage Carter's service needs.

KAREN: It's so hard to be poor. I [used to] look at it academically. We live that now...living off less than $\$ 20,000$ a year for a family of three because if you make more your SSI get's cut...don't earn too much because you're in the loophole where you're doing worse because you're making too much money...try to play that game for a while until you're desperate for your kid to go to school so you can go back to work, use your master's degree and teach everyone else's kids."

\section{Raising Carter in a neurotypical world.}

Accommodations for Carter's sensory issues at 12 months before diagnosis.

When Carter was turning one Karen recognized his need for supports for his sensitivities to sound. When her graduate school friends came for a visit just before his birthday, Karen had a "practice party" with a cupcake and candle (Figure 18). Her friends thought it was cute but Karen saw it as a necessity to make Carter's real party successful:

KAREN: He need[ed] it or he [would have] fallen apart at his birthday when 
people started singing the birthday song. He probably [would have] freaked out because it's loud...He was always very sensitive to noise. And so that's what [the picture] was about...Yeah we had a good practice birthday party...This is one of my favorite pictures that he and I. He's so happy...

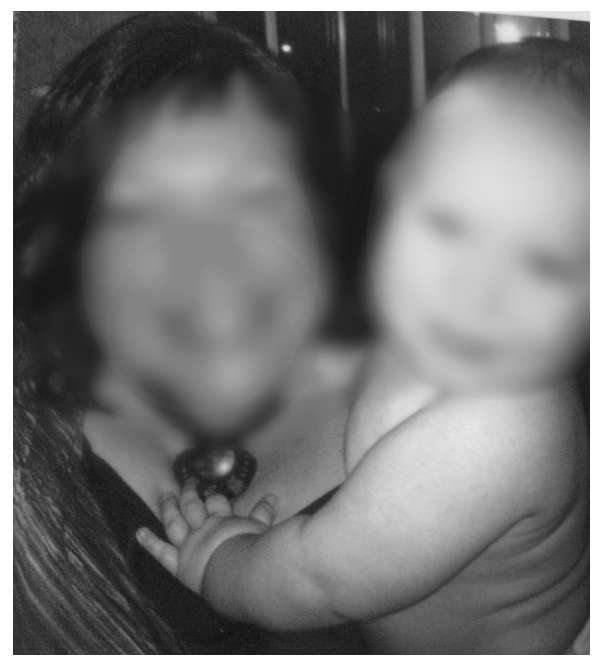

Figure 18. Photograph K-4. Karen is holding Carter at 12 months. He was still engaged in the world. Karen was struggling with sleep deprivation and postpartum depression.

In this photograph Karen is dressed up and smiling at Carter's practice birthday party. When I commented on how nice she looked and what a great picture it was Karen told me "this is the face of postpartum depression." Her comment surprised me. She looked like a happy mom to me. As we talked I came to realize how much this photograph represented to Karen and what a difficult time in her life it had been caring for a high needs baby and not having a diagnosis.

KAREN: It was the first time I had put makeup on probably since he was born...and definitely one of first times I had been dressed in weeks. I remember...I went and got a haircut because I had people coming. But this is like the face of postpartum depression...I'm totally trying to hide it. And I was happy that my friend was taking the pictures... and I And I mean it's a beautiful picture and if you looked at it I think you would never know. But...I was miserable... I can still see dark circles under my eyes in this picture...It was so, so hard having a high needs child and he didn't have any sort of diagnosis at this point. 
Seeking autism eligibility through educational services. It wasn't long after Carter's regression at 18 months that Karen sought an autism diagnosis. She was still receiving doubts or reassurances from providers. After a long developmental evaluation with the education service district, the evaluators assured her that while Carter had developmental problems, he did not have autism. However, their reasoning did not sit well with Karen:

KAREN: As they were leaving they said '...I would not worry about autism ...he is not at all autistic. He...isn't presenting any autistic behaviors. [Carter] smiled at us. He looked us in the eye.' ...As the second evaluator was leaving... she said, 'I know he's not autistic. I can tell.' ... Then she said this- this always sticks with me because she said '[he's not autistic] because I can tell he's intelligent. There's intelligence there..."And then as soon as she left my husband was... kind of relieved too and he's like 'OK they say he's not autistic.' [I told him] 'Did you hear why she said he wasn't autistic? ...because he's intelligent?' And I'm like "autistic kids can be totally intelligent...they're like any other kid and they can be gifted...'

Navigating services. Karen spends the greatest amount of her time piecemealing services, researching funding options, and planning ahead so critical services are not interrupted due to factors outside of her control. Karen shared a dramatic example of the need for parents of your children with autism to be on top of all services all at once. While Carter has almost no speech, Karen found out almost by accident that he is hyperlexic. At age three Carter began using magnetic refrigerator letters to spell words and create sentences. Carter's pediatrician confirmed his hyperlexia. Once recognized, Carter's precocious language skill helped him interact with the world and regulate his behaviors better. When Karen realized he was hyperlexic she worked with the school district's speech therapist to get an augmentative communication application and an iPad. She described it as a "fight" to get the education district to provide the augmentative 
communication services and device. She said she "went to them time and again saying he can read but he doesn't have the motor skills to write." She said at that time he didn't even have the motor skills or point action to write in the sand. Karen researched and found the best program for Carter's needs This technological tool became Carter's connection to the world.

KAREN: He uses it between home and school. He uses it in the community. That's how he communicates. He has a few signs. He has no words, whatsoever, but he's hyperlexic, so he can spell. He's been able to spell since he was two years old. So, he spells a lot and also these devices have shortcut buttons. So for things he asks for often...[He] Mostly requests. He's a big requester, so he can let us know he's hungry.... or his favorite foods to eat. He lets us know, a lot of times that he's all done with something. You know, he'll say all done when he's all done eating or he's all done with this game or he's all done with this video he's watching. He's moving into being able to share and all of that stuff. And... like if you have a kid that at first they're saying drink, or whatever. Then they're able to say I want a drink. He's starting to be able to put sentences together.

When Carter turned three the education service district needed the iPad back because other children in the EI program needed the device. He had aged out of the EI and was transitioning to the early childhood program. There was no transition for communication goals planned. Karen had to work to maintain Carter's connection to the world. She found out that there were devices available through the county program but there was a long wait list as all were in use. The days passed and soon her son was about to lose his way of to communicate. Karen went to the developmental pediatrician but he felt "If you do [give him an augmentative communication device], he'll never have any incentive to talk." Karen had to advocate for Carter. She said, "So I had to pool the research and be like 'that's not what the speech pathology research said."' Then she said, "How would I do this if I hadn't gone through an [education master's] program?" 
Karen eventually figured out a way to bridge the gap between EI program needed the taking back the iPad and the wait list for a new augmentative communication application. Despite being economically stretched thin, she and her husband scraped up enough money to buy a used iPad. Then, after researching communication applications, she found there was a free trial for a few months. Karen was able to get the free trial and that got them through until the early childhood special education services approved the $\$ 200$ cost of the application. It was stunning to think that without Karen "fighting the battle" then Carter would have lost all communication access for 6 months. At that time Carter had engaged in self-injurious behavior when he was frustrated. Karen told me "It's not sensory. It's not autism. It's that he can't communicate."

\section{A new way of life.}

Emotional exhaustion, loss, lack of support, and other nearly insurmountable obstacles marked the first part of Karen and her family's journey to diagnosis and autism services. The second part marked a change. With a diagnosis, the internet support, and an understanding of available service system Karen began to have a sense of stability and soon after felt successful as a parent. She often still had to manage unpredictability and the effects of autism on her son and their family life, but Karen had a sense of selfefficacy and that translated directly to her feelings of success as a mom.

Interviews 2 and 3: The last 5 photographs. During our third interview Karen told me that she didn't realize when she selected the photographs that they represented "before and after." Photographs 6 through 10 represent the life that she has now and also how far the whole family has come since Carter's birth. The last five photographs 
represented a new era in their lives. They had a diagnosis and services in place for Carter.

It was hard managing services and his sleep deprivation but they'd arrived at a destination.

The last five photographs (Figure 19) reflected a change in Karen, too. She began to feel confident as a mother and successful as a parent. She understood Carter's needs and each photograph shows Carter engaging with the family and the world. Each picture also includes accommodations that Karen and her husband figured out so that Carter could participate in family activities. Karen described one of the photographs as a "triumph of parenting" because she was able to help Carter engage in the world and experience happiness. (see box display, Figure 19) summarizes the significance of each of the five photographs with descriptions and transcript quotes.

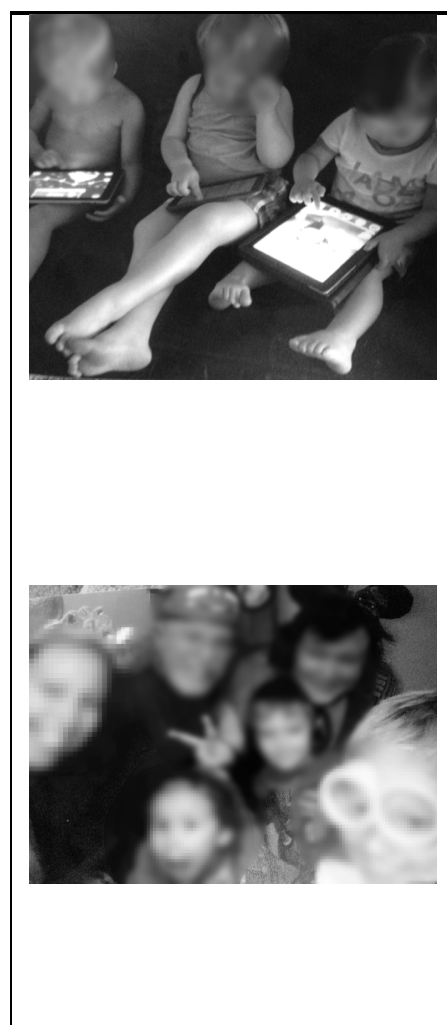

Photograph K-6. This photograph was taken during a trip to visit family in California.. Carter loved the plane ride and was comfortable and happy. The picture depicts Carter with his cousins, all of them sitting together on their own just being together. "You know. He seemed just totally typical then. And so I really love that picture. Not because he looks typical but because he's ... able to enjoy something without, like, a bunch of accommodations... The thing is I feel like other families get to do this stuff." Karen had told me in the past how the iPad helps Carter regulate. This photo suggests it bridged social interaction too.

Photograph K-7. A family picture with Karen's sister's family on a trip to Disneyland. "Yeah. It was our first... We had just gotten there, like, off the plane and it was our first night. So we were just all real tired and silly and messing around. It just reminded me of my sister and I we were little, we used to do stupid stuff like that..." Carter is wearing his headphones in this photograph to help modulate sensory processing problems. 


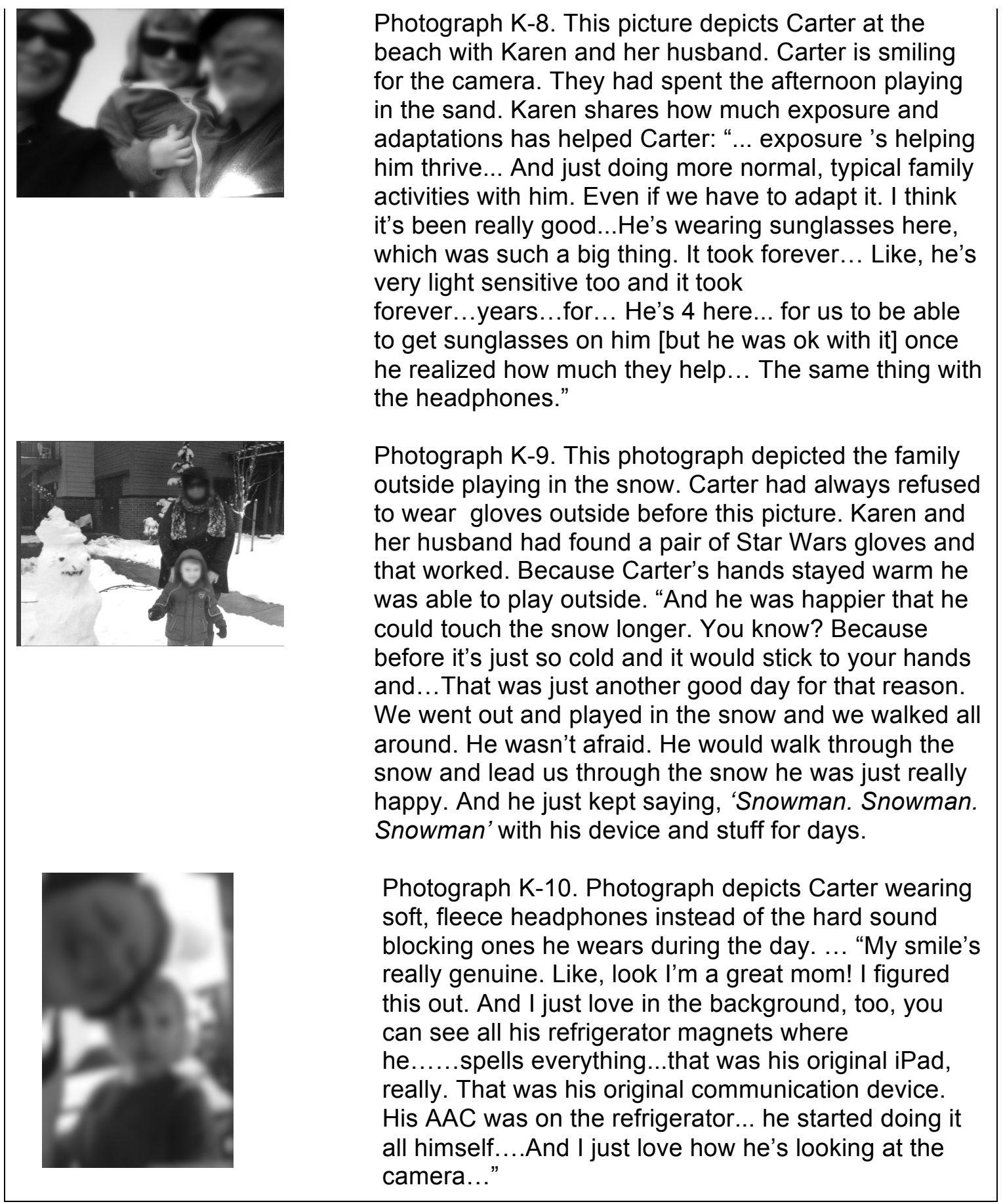

Figure 19. Box Display. Karen's last 5 photographs marking the end of her journey from diagnosis to services. Karen reported these photographs represented of parenting joy and accommodations to help Carter engage in the world. 
Summary. Karen described intense early experiences as a mother. These may not have been unique to autism but her resilience and love for her child helped her move into an empowered parenting experience. Like the other participants she navigated difficult service systems and weathered frustrating and exhausting obstacles. After the study was completed Karen sent me an email (Figure 19). I considered her correspondence important to my bracketing process. To me, her email was clarification that despite the journey, she is a happy mom and loves (and has loved) her child every step of the way. She also sent a final photograph to include in the paper:

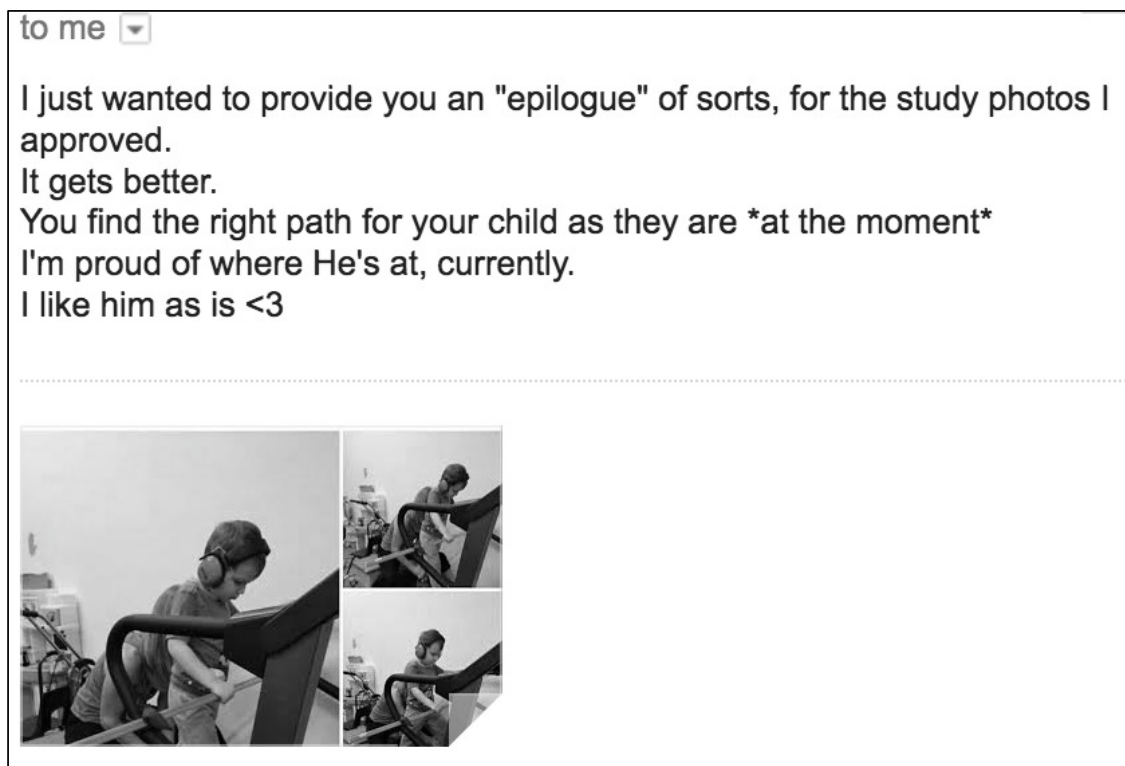

Figure 20. An email Karen sent to me after the completion of our interviews. She wished to add to her story with an epilogue to the study photographs. 


\section{Section Two: Essential Themes}

\section{A Narrative Connected by Temporally-Bound Essential Themes}

\section{Introduction}

As a phenomenological researcher I was concerned with how each participant conducted their daily lives (Gubrium \& Holstein, 2000). Welman and Kruger (1999) describes descriptive phenomenology as a method that allows for understanding the phenomena from the social and psychological perspective of the participants. I examined and explored narrative and photographic the data during each phase of the process. I also engaged in reflective activities and data analysis in order to "allow the themes to emerge from the data." I employed the process of looping back (Dahlberg \& Dahlberg, 2003), engaging in a constant comparison analysis. In doing so I followed and coded strands of lived experiences until I arrived at those essential themes that formed the structure of the phenomena. I repeatedly wrote a reflection in my journal, "the essential themes will float to the top of the data." This concept was a guidepost to me. It was a metaphor that helped me makes sense of the phenomenological data analysis and understand the ways in which the essential themes answered my study's research questions.

In addition to ongoing bracketing and reflection activities, I spent the months following the conclusion of data collection analyzing the data by looping back and insuring that the essential themes were true for every participant. Then I returned to the transcripts, coded them again and confirmed that I hadn't missed any essential themes. Throughout this process I consulted and debriefed with a phenomenological expert, refining my interpretations down to essential themes. Near the end of the study I shared 
the essential themes with the participants through a group member check meeting. All participants expressed that the essential themes accurately represented their lived experiences. According to phenomenological theory, the essential themes should be recognized by anyone experiencing the phenomena (Vagle, 2014). The parents confirmed that I had captured their lived experiences.

The essential themes formed a temporally bound model of the phenomena. The participants had been on a journey from the early days when concerns for their child began to emerge to the final phase of securing and navigating services. I journaled and contemplated the concept of emigration: Emigration is "the act of leaving one's own country to settle permanently in another." (Merriam-Webster, 2017) The concept of leaving one country (i.e., lived experience) matched my interpretation of the phenomena. While parents of children with autism did not "choose" to embark on the journey from their expected parenting experience, they nonetheless did. And it was a long, unknown quest from first concerns to diagnosis and specialized autism services.

\section{Sources of data.}

Participant interviews and photographs. In phenomenology, the participant's interviews are the raw narrative data (Gorgi, 2009). In my study this included participants' stories about everyday life and reflections about their experiences as a parent of a young child with autism. The raw data for my study also included the photographs (i.e., the images and the participants' descriptions of associated significance, memories, and feelings). As my relationship with participants grew they sent me emails with thoughts about the study, corrections, and links to articles about autism. These 
became part of the participant data.

Researcher activities as data. Phenomenology also includes research activities as a part of the data set (Vagle, 2004). My engagement in bracketing, reflection, and data analysis contributed to the reduction of data to lived experiences and essential themes. As mentioned earlier, bracketing is central to descriptive phenomenology. It is a researcher stance as well as ongoing activities that reduce the influence of interpretation on the data (Vagle, 2014). From a strictly Hurselian (1970) perspective I viewed my search for the phenomenon as a mathematical metaphor, excluding internal and external bias and supposition. My bracketing activities began before I embarked on my research activities. I selected phenomenology and ecological theoretical lenses in order to guide an investigation that acknowledges external influences on development and parenting. My literature review also sought to neutralize the effects of discourse and dominant views about autism. My activities as a were linked to the data I collected. As the study progressed I used reflective journaling and repeated engagement with the data. I returned to the participants' on several occasions when I realized I was superimposing my own interpretation of their experiences. Participants engaged in dialogues that corrected my misunderstandings through email, a group meeting, and clarifying conversations.

A timeline of the phenomenon. As each participant shared their lived experiences, several robust essential themes emerged. Early in the research study, parents laid out a temporally bound structure of their lived experiences. The essential themes had a loose chronological framework shared by all participants. Each participant described having concerns and despite seeking help, they little validation or support from providers. 
Parents spent an average of 22.5 months from first concerns to receiving early intervention support and another 10 months before obtaining autism-focused interventions. The graph in figure 20 illustrates the shared experiences from participants' first concerns to commencement of autism services for their child. The essential themes emerging from this study's data all clustered around lived experiences during the lag time. This created a temporal framework for understanding the phenomena of parenting a young child with autism receiving early special education services.

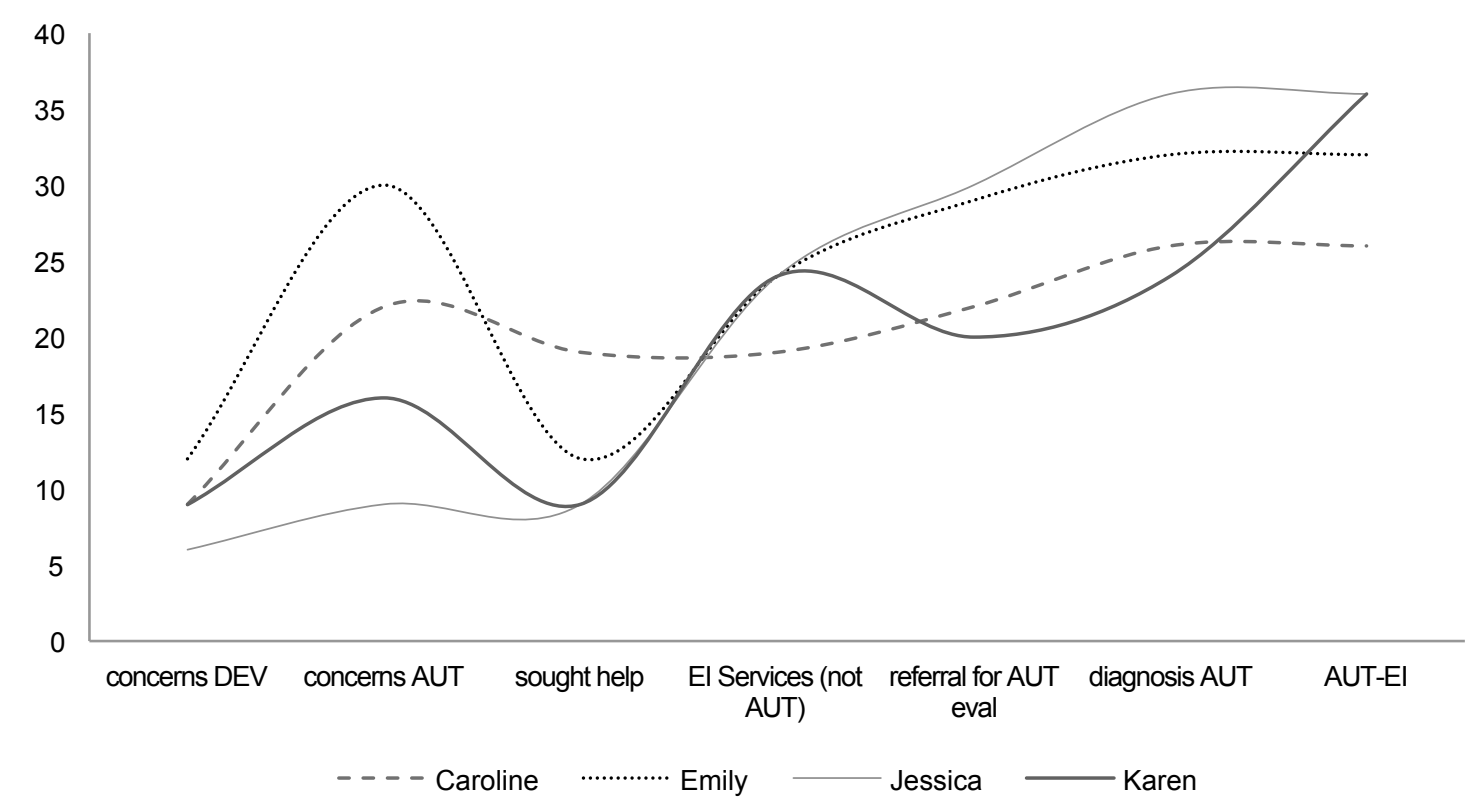

Figure 21. Graphed representation four participants' journeys from first concerns to autism diagnosis (in months). All participants had concerns for their child's development around their child's first birthday and secured autism services around their child's third birthday. The graph represents the events between 12 and 36 months.

Early on parents sought help from professionals and all were reassured that there was nothing atypical about their child's development. This marked the beginning of their journey. Their experiences during the first three years clustered into essential themes 
experienced by all participants. The final landmark in the participants' journeys was starting a new family life with services and support. Participants had forged their way to autism diagnosis and services. There had not been a clear path. All parents navigated their way using their intuition, resiliency, ingenuity, and hope. They, in turn, helped pave the way for families making the journey after them. The essences of this journey manifested across all participants: (a) pioneering; (b) making the journey as a family; (c) navigating the undeveloped new world (AUT-EI service systems); (d) overcoming obstacle and challenges; (e) ingenuity, resilience, and perseverance; and (f) reflecting on the first three years and looking forward (birth to 5). Each essential theme is discussed later in the chapter.

\section{Essential Themes}

The following six subsections presents the essential themes across all participants for the phenomena of parenting a young child with autism receiving early special education services. A discussion of each will be supported by passages the participant's own words extracted from the interview transcripts.

Essential theme 1: Parents as pioneers. Lag time between participants first concerns about their children's development and autism diagnosis was one of the most salient themes. All parents were concerned about their children's development prior to their children's first birthdays and their children were not diagnosed with autism until close to three years old. All four parents reported experiencing invalidation in a variety of forms (i.e., dismissal, reassurance, or skepticism) as they sought help from professionals, friends, and extended family. Parents sought help in a world based on neurotypical 
development and had to forge their way in order for their very young children's differences to be acknowledged and addressed. Despite often encountering doubts by doctors, family and friends, participants pressed on and continued to research and advocate to secure help for their child. This section discusses the essential theme Parents as Pioneers. There are two subsidiary themes (a) parental recognition of early autism markers and (b) invalidation from others.

Parent recognition of early autism markers. All four parents reported having concerns for their children's development by twelve months of age. Concerns clustered around sensory issues and social communication. Jessica recognized differences in sensory modulation and "maybe six months old that [Helen] might be autistic....[During Gymboree class] Helen just shuts down and sleeps." Like Jessica, Karen noticed that early on Carter had sensory processing problems. She said he was "super sound sensitive" even when he was a baby, at six months old they walked into a party, "it was a wall of sound...and [Carter] just lost it." Emily had two older children and noticed Joshua's engagement and language was marked by acquisition and loss of skills. She told me, "he was losing skills and his I was like 'that was really weird.' And he would do something really cute or really smart and I could never get him to repeat it..." Caroline reported that she "kind of knew from birth," that something was different in Nathan's development. When Caroline and her husband filled out the ASQ she said, "we were like oh my God. He is not even on the charts for three of these six categories."

Invalidation from others.

Pediatricians. All four parents reported that their first step in seeking help for 
their child was to talk to their pediatricians. Strikingly each parent was met with a similar reaction from their providers when they voiced concerns about their children's development or autism specifically. Pediatricians reassured parents or invalidated their concerns for their child's development. Table 2 presents examples of responses each participant received from their child's pediatrician.

Table 2

Participant Reported Response from Pediatrician About Concerns for Child's Development

Participant Pediatrician's Response

Caroline
[After failing the developmental screening] we called [his
pediatrician] and we're like 'we would like a referral.' And they're
like 'well he--you know he just had his 18-month appointment. Let's
wait until he's 24 months.' I'm like [no way!]
He's a really old-fashioned doctor... he thinks [autism] over diagnosed
and over treated. He thinks 'oh everyone thinks their kid has autism.'
Emily
They weren't concerned, they were looking maybe some lack of social
[skills]. They said language age delay, but then they said you [are
from another country] and this is English, and we don't know if the
delays are due to the bilingual education. I'm like, 'no, no, I don't
think so'...I had to keep pushing...
[After one pediatrician recommended EI Karen's regular pediatrician
said] 'I don't think that... that's really early....some of the things
you're mentioning are actually a 12 month milestone, not a nine
month. There's nothing wrong with you baby. You don't have to worry
about it.'

Other providers and friends. Providers and friends also reassured participants that their children did not have autism. These opinions were based on experiences with older autistic or typically developing children. When Jessica expressed her concern that Helen had autism to her early interventionist, the interventionist said "Oh my son did that too and [he's not autistic]." Caroline's family based their opinions that Nathan didn't have 
autism on their experience with Caroline's sister who has "classical autism" and is severely impacted by it. Compared to her stepsister with classical autism, Nathan "can engage and has more language than when [my sister] was little.” Karen reported that other people assured her "...there is nothing wrong with this kid like look he's looking [at me] and he's playing with me....[or] he's walking on his flat feet. He doesn't flap. He doesn't line things up."

Emily elaborated on the issue relating an interaction with an acquaintance who meant well saying, "my kid didn't talk until ...4 and [now he's] in graduate school." Suggesting that this experience of invalidation is common, Emily also told me about a coworker that she hardly knew offered this opinion "[She said] 'I think Autism is a madeup diagnosis. I think kids can act the way they choose to act. "' Emily told me her sentiment towards this attitude was: "I was like, you obviously don't have any children, do you?"

Effects of invalidation. The effect of invalidation about concerns was complex and across participants it ranged from frustration, desperation, and worry. I asked Jessica what it felt like when service providers didn't agree with her. She waited a moment before she answered, "doubtful." For Jessica it was conflicted; on the one hand she thought "maybe [Helen] will be fine." She felt almost optimistic. Another side of her doubted herself thinking, "Do [I] really see this?" She said her "knowledge and instinct...meant nothing basically.” Karen also doubted herself sometimes, “...I'd be like OK, OK--then I'd just think 'I'm just being paranoid.' I was [starting to feel like] ...a Munchausen mom like I was looking for something." 
At the universal member check meeting the participants explained that the worst aspect of invalidation is the blaming that takes place. They shared their feelings about research articles citing reason after reason that the mother's behavior during pregnancy or the early years caused the autism. Karen said that is what makes her "the most angry." Emily reported feeling angry when a female coworker told her to feed Joshua a special diet and "that was all he needed [to get over autism]." Caroline expressed her sentiment towards individuals who say her son's issues are parenting inadequacies, "people [say] 'well you know it's your parenting' and it's like 'yeah well you take him for a week and you figure it out. I'll sit back and watch.'

\section{My reflections on the essential theme parents as pioneers.}

My journal entry April 10, 2017.

Returned from meeting with the participants for the universal member check. Why had they taken such a strong position against the original metaphor super parent? Had this reflected my preconception or bias? It seems so. Everyone one of them concurred that they found it an inappropriate description of their experience. They found the pioneer metaphors more fitting. Karen stated "I didn't like being called a super parent. I don't see myself as any different from any other parent." Caroline added "yeah. We do what we have to do. We're just parents. I know lots of families who have it a lot harder than I do. I'm just a mom not a super parent." Emily and Jessica nodded in agreement. I acknowledged their feelings about this topic then asked "Does pioneer seem like it fits." They said it did.

Summary. The retrospective nature of interviewing participants about early experiences allowed a comprehensive depiction of the early experiences with their child with autism. Each parent noticed developmental issues with their child long before friends, family members, or professionals. The participants experience invalidation from others before the manifestations of autism became noticeable to others. 
Essential theme 2: Making the journey as a family. Following my first round of interviews I recognized a pattern emerging; autism and family life were inextricably woven for participants. Caroline's comment captured this aspect of her lived experience, "you cannot separate autism from parenting." The other parents echoed her sentiments. Having a young child with autism informed every aspect of family life. The essential theme, Making the Journey as a Family, is comprised of two subthemes: (a) Belonging and the Family Unit, and (b) Bridging the Relationship Between Autistic Children and Extended Family Members. Each will be discussed in the subsections below.

Belonging and the family unit. All of the parents in this study described making the journey from initial concerns to autism diagnosis as a family. This included a sense of belonging and connection. Caroline had a keen insight into the way Nathan sees the world. As a person with autism herself she related to his experiences. She said that people often think Nathan is being "stubborn" or "defiant" but she told me that wasn't the case.

CAROLINE: ... his brain is structured differently. We're in the same reality as everybody else but it lands for us differently. And our nerves pick it up differently whether it's tactile or [other autistic traits]...and that's the funny thing is my son is definitely an apple from my tree. His sensitivities are the same as my sensitivities...

Emily and Karen too commented on the "likeness" of their children to themselves or their husbands. In Emily's case, she half-joked that her husband may be "on the spectrum." Emily's view of these characteristics was positive and inclusive. While her husband experiences social anxiety and can be somewhat introverted, there is a strong relationship between her husband and their children. His love of Star Wars connects the family including Joshua. Joshua, his siblings, and his dad love the Star Wars movies. 
Emily told me "...they're obsessed [they all have] costumes and dress up every time there's a movie." I spent some time reflecting on the role of Star Wars to her family. In some ways Emily described it to me as a restricted interest and maybe as she said a "spectrummy" trait. It also struck me as a bridge between the family members. I wrote in my reflective journal "Star Wars is a context for all family members. It is an identity of sorts...one that allows all family members to [feel a sense of belonging without a social construction of ability]."

Karen's perspective reinforced this essential theme's validity. She told me that her she and her husband are "total nerds." She said they both have social anxiety and it runs in the family, "we just like concentrated those genes into this kid." She jokingly recollected that someone once told her "two pit bulls can't make a poodle." While Jessica didn't describe shared personality traits or characteristics with Helen, she confirmed the essential theme "belonging and the family unit" in the way she described family connection. To her, "the most important thing is that this little family understands each other and supports each other. Caroline's sentiments echoed Jessica's, "the people in our family get what they need or what we have available to give them. Sometimes or resources...are limited to what they are." Belonging amongst immediate family members may act as a moderator of the effects of lack of understanding and invalidation from those outside of the family.

\section{Bridging the relationship between children with autism and extended family}

members. The participants reported that while they and their husbands were on the same page and their immediate family unit was strong, sometimes other family members were 
skeptical or confused about autism, particularly the older generation. Participants described their role as advocating for their child and bridging the relationship between their autistic child and extended family. Some family members disagreed with an autism diagnosis. Caroline said her parents questioned, "How can [the doctors] tell? He's so young. He talks, hugs and he's very affectionate. He has some language." Emily felt distant relatives didn't see Joshua enough and they thought he was too young for a diagnosis. Family members told her that "[Emily and her husband] were making a big deal out of nothing." She told me that her mother-in-law said "back in the 70s when I was raising a kid no one really cared about that kind of stuff" and "everybody's kids didn't say much and [that was] fine." Both sides of the family expressed disagreement with Emily and her husband, “don't even try to get him tested. They're going to try to force medications on him." Both sides were also "very against [them] having anything to do with Joshua [being] on the spectrum." Jessica's comments reinforced the theme. She told me "we have a struggle with some family members that are still in disbelief." She said those family members insist Helen is "OK" and that she and her husband need to "let her grow up...you guys are too soft on her." While Karen did not see her parents often due to geographic distance, she told me about her father's reaction to Carter's regression. Her father did not necessarily express doubt but he was concerned about the dramatic change in Carter after the regression. Karen helped her dad understand Carter's autism. Her dad asked "What's wrong with him. Is he sick?" Karen broke down and said "that's how [Carter] is now. No one can tell me what's wrong with my baby." That conversation began a dialogue with her father about Carter's autism. 
While participants describe their roles advocating across many situations, I considered the unique type of advocacy with extended family members. Participants acknowledged the importance of family traditions and also wanted to foster closeness between their children and families. This nuanced difference between advocacy with society and family was a strand in the essential theme Making the Journey as a Family.

\section{Essential theme 3: Navigating the undeveloped new world (Autism-EI service} systems). The essential theme Navigating the Undeveloped World of Autism Services began with parent's first concerns and ended with early childhood services (i.e., after age three). This lived experience correlates with the "early intervention service period" (i.e., birth to age three). The parents in this study navigated a largely undeveloped world of service systems for infants and toddlers with autism. This section discusses the essential theme Navigating the Undeveloped New World of Autism Services for Young Children. Following a brief introduction, the remainder of this section reports on four essential subsidiary themes (a) Intersecting Service Systems, (b) Autism Health Service Systems, (c) Early Intervention Services, and (d) Social Media Support and Navigating Services.

Introduction. Service and treatment for very young children (i.e., birth to three) with autism are emergent areas of practice (Rogers \& Vismara, 2014; Zwaigenbaum, 2015). As discussed in Chapter Two, despite early markers by 12 months and diagnostic tools for a valid diagnosis at 18 months, most children with autism remain undiagnosed until after their third (Shattuck et al., 2009; Zablotsky et al., 2017). Participants in this study struggled to get their children diagnosed with autism. They began seeking help when their children were around 12 months but none of the service systems offered a 
clear roadmap for families until the children reached ages three or four years. Once diagnosed, the service systems were not prepared to treat autism as it presented in the first three years of life (e.g., emergent social-communication differences, sensory processing problems). On average it wasn't until children in this study were 32.5 months that specialized autism services became available. Table 3 displays the parent's concerns and wait times for services and diagnoses (i.e., developmental delay then autism).

Table 3

Participants Concerns, Wait times, Services (in months)

\begin{tabular}{lccccc}
\hline Participant & $\begin{array}{c}\text { Age of dev. } \\
\text { concern }\end{array}$ & $\begin{array}{c}\text { Sought } \\
\text { help }\end{array}$ & $\begin{array}{c}\text { EI Services } \\
\text { (not AUT) }\end{array}$ & $\begin{array}{c}\text { Age of } \\
\text { AUT } \\
\text { diagnosis }\end{array}$ & $\begin{array}{c}\text { Age of } \\
\text { AUT } \\
\text { services }\end{array}$ \\
\hline Caroline & 9 & 19 & 19 & 24 & 26 \\
Emily & 12 & 12 & 24 & 32 & 32 \\
Jessica & 6 & 9 & 24 & 36 & 36 \\
Karen & 9 & 9 & 24 & 24 & 36 \\
Mean & 9 & 12 & 23 & 29 & 32.5 \\
\hline
\end{tabular}

Intersecting service systems. All parents reported that they first sought help from their child's pediatrician. At nine months participants began feeling concerned about their children's development. By 12 months they expressed those concerns to their pediatricians. Participants did not receive specialized autism services until 32.5 months on average.

All parents in this study described stress and frustration associated with navigating and coordinating services. During our first interview Caroline said that because the health and education services are not coordinated, "[the system] is really failing our kids in major ways." She added, "if you aren't keenly aware of how the 
systems interact or not completely organized and understanding your rights [you will not get the services your child needs]...I have to be the one driving it and be on top of people." Karen's experiences were similar, "you can fall through the cracks... It is a constant battle because you're constantly approaching these people and saying 'ok you're supposed to be doing this and you're not...the time I spend--hugely-- is navigating [services]." Karen said this involves being "the squeaky wheel... [you] play phone tag and research what resources are out there." Jessica described her work with providers as “...to keep pushing” until she got the help Helen needed. Emily summarized the issue with the following story about calling to apply for ABA services for Joshua:

EMILY: I called every day for two weeks until someone finally called me back, and then they were like 'oh, you need to go and do this with your developmental pediatrician, ' who is impossible to get a hold of.

As I reflected on the lived experience navigating service systems, I contemplated what it must feel like to call to get help for your child every day for two weeks only to be told you had to start from the beginning again. As I began analyzing data, I made a table (Table 4) of all the services the participants navigated on a regular basis. As Karen explained when one service is interrupted, it has a cascading effect on the rest. When I looked back down at the notes in my journal, I was surprised to see the number of services participants coordinated and managed on a regular, often daily, basis. They were all related and intersecting, but the players didn't communicate or coordinate. That fell on the parents' shoulders. 
Table 4

Services Participants Were Receiving At the Time of the Study

\begin{tabular}{lcccc}
\hline \multicolumn{1}{c}{ Service } & Caroline & Emily & Jessica & Karen \\
& & & & \\
\hline Pediatrician & X & X & X & X \\
Developmental Pediatrician & X & X & X & X \\
Other Medical Specialist(s) & X & & & X \\
Speech Therapist & X & X & X & X \\
Occupational Therapist & X & X & & X \\
ABA Program & X & X & & X \\
Early Childhood Education Program & X & X & & X \\
Private Preschool & X & & X & \\
Preschool SPED Consultant & X & & & X \\
Respite Worker & & & & X \\
Oregon Health Plan Services Other & & & & X \\
Housing Services & & & & X \\
Augmentative communication specialist & & & & X \\
Autism specialist & & & & \\
\hline
\end{tabular}

Navigating service systems. All participants coordinated and navigated services

systems for their child. They stayed on top of wait lists, unexpected service interruptions, or other service problems. Each service system had its own procedures and requirements and none of the systems communicated. Karen's comments summarize the essential theme Navigating Service Systems:

KAREN:...when the year changes, it's all new approval because they only give you $\mathrm{X}$ amount per year...to make sure you're not going over. [At the end of the year] they just cut you off, start from scratch. Then you get kicked out of the system. We just got an approval on December 28th. [It] doesn't mean anything...sometimes we were already getting services, and then they would basically get cut off. That's what's so hard. You've already planned your life...He used to go do speech every Friday at 11...that's how our life was set up around that. Then, 'oh, they cut you out.' You lose that spot and then they say 'we need to get it approved, and that takes two months. [When they finally approve they say] 'Okay, well it looks like your therapist is scheduling out six months from now'... how do you live your life like that? 
Emily also experienced wait times and ways to work with the system to get services for Joshua. Emily told me, “[The developmental pediatric clinic] didn't call me for two month" and when they called in December, the office told her "we're booked out until May." Emily called weekly about cancellations. The clinic finally told her "quit calling." Emily explained that she had done a lot of research and the typical wait for an autism evaluation with a developmental pediatrician is six to 12 months. She said, “...but I mean like “...everybody does [have a wait list]...I was on the blogs and everything —or the Facebook...for autism. OHSU has [a waitlist], Footprints, everybody that does diagnosis has a six month wait—six months to a year.... to get tested to see if your kid's on the spectrum..." Once Emily secured services for Joshua Emily said she knew "little tricks" and Joshua received speech therapy twice a week for a few months so he could catch up on what he'd missed while on wait lists. The clinic eventually realized what Emily was doing and told her she couldn't bring him twice a week anymore." She reasoned I felt ... that he'd been so long without services that I was like "uhhh ok he really needs this" and it was helping so much. It was going from completely nonverbal to actually talking and now he talks in 3 or 4 word sentences which is AMAZING for him because a year ago now, he was completely nonverbal..."

Karen too felt uncomfortable with the wait list for evaluation through the developmental pediatrician so she called a speech therapist. "So we were able to get into speech... I remember we called in May...and I was able to get in for the speech evaluation in September which was still a long wait but I was able to do that." Caroline told me that she "went behind the pediatrician's back and called for a hearing test." After the hearing 
test Caroline looked at the audiologist and "WE-NEED-HELP!"

Karen's insurance covered ABA but there were no providers available in network. When she couldn't get a call back or help for Carter, she went to a local newspaper, "[After] I went to the media, I got a call the next day [from OHP] that he was approved... after fighting them for a long time."

Jessica told me that Helen's speech services were abruptly terminated due to an insurance administrative error, "[we had to] pull her out immediately and stop service....and try to get a referral." After that Jessica pushed the doctors to evaluate for autism “...it's more like I push them to diagnose.... if it is autism...we can have OT and speech ... covered."

Towards the end of the study Caroline wrote an email to me telling me that Nathan had been "dropped from ABA" but placed in a preschool for children with autism and he loves it. "Just keep this in mind "she wrote "how completely complex a life with a special needs child truly is. And ours isn't even THAT complex if you ask me :D”

\section{Educational services.}

Parents were largely dissatisfied with EI and ECSE services. Overall, the participants were not satisfied with early special education services. They all expressed there were not enough funds to make EI effective. Caroline felt the EI system was "a completely ineffective model of services" and the services they were getting was "abysmal." When Nathan was finally diagnosed with autism at 24 months he "[got] 12 visits of speech per year and 8 visits of occupational therapy." However, Caroline said

"we never saw a speech therapist or occupational therapist...and we didn't push it....but 
we're not even getting what we're entitled to."

Jessica's was also dissatisfied with educational EI services. Jessica found it frustrating that the interventionist was her only point of contact with the EI program "[I] didn't know who else to talk to." All of Helen's educational services before age three were delivered in the family home using a coaching model. She wanted an autism evaluation but instead received EI coaching. Jessica reported "I'd been telling [the EI coach]...look this is a very autistic behavior like lining things up..." The coach did not refer for an evaluation instead offering, "well my [typically developing] son was like this... "Jessica told me this was frustrating, she said "I'm like 'OK well [that] might help [him] but it really doesn't help my daughter."' Jessica also felt the parent coaching activities were of little help, "I would say [EI was] meaningless ...but then [maybe not for some] depending on what your goal is." When Helen transitioned from EI to ECSE Jessica remained dissatisfied with the amount of services, “...it's like a very minimum you know so... we, we don't necessarily rely on it."

Emily received EI services weekly. However, the coaching model was not what she'd expected and she found the approach out of touch with her family situation and at times condescending. She told me "I was on the floor with [the coach] and [Joshua] for an hour every Wednesday and she would be like 'No. You're doing it wrong. you need to do it like this..." Emily said "[the interventionist] used a lot of vocabulary that I didn't understand and I was like $O K$. Whatever." Emily had early intervention visits on Wednesdays, her day off. She joked “I hadn't showered, I was in my pajamas.” Emily remembered the interventionist asking her " How often do you get on the floor and play 
with your child? "' Emily told her, “"Well not that often, I'm kind of busy." Emily felt like it was a "guilt trip."

Caroline expressed that the educational providers were "stretched too thin." She said she "loves the educational [providers]...but we need more from them and they need more [resources] to do a good job." Caroline said the educational approach "is much better" than the medical because "their hearts are in the right spot." When in-home EI started Caroline was sent an occupational therapist to provide speech services. The IFSP service hours were never met.

Karen received in home EI for Carter. Since Carter would not engage when the interventionist was there, instead Karen received coaching on things to do during times when Carter engages outside of EI visits. Karen said the EI coach advised “...so when I'm not here and he actually wants to participate, you can read to him like this. Or you can play these kind of games." Karen was supposed to get 17 visits with the intervention coach. She told the coach, "if you only come once a month, he's not going to get seventeen visits. We're halfway through the school year and we're not even close to halfway through these visits." The interventionist was surprised by the number of service hours in the IFSP. Karen recalled, "She was like, 'seventeen? There's no way I could do seventeen visits with my caseload...I've never seen a kid with seventeen before. That's crazy to me. There's no way I can do it."'

Ancillary benefits of EI for autism. While parents felt EI was ineffective or insufficient due to lack of funding, they nonetheless chose to participate in EI services. Emily said "some parents just drop it" for the reasons she described. However, the 
participants in this study saw EI as way to prepare for kindergarten and "get their foot in the door," and give them a chance to "practice IEPs." Jessica described the main focus of EI / ECSE as "[being] in the system is so that [Helen] can have a smooth transition to kindergarten public school. Karen accessed EI services in order to get Carter's augmentative communication system set up prior to preschool, "That's what it is [EI to get foot in the door for services]. For us, we were able to go around the developmental pediatrician, who wasn't supportive of alternative communication."

Caroline wanted the autism educational eligibility in place before kindergarten. She explained "there's a separate pot of money and there's more services if they have this autism designation." She told me that if they go into kindergarten as "autistic," then they are eligible for those services immediately. Emily said she wanted to "just bail on [the early interventionist]" but she knew "he needed the services, and I knew that it would open more doors. I knew enough to know that if I want to get him in a special preschool or whatever, then I'm going to need to keep up with this," Caroline felt the amount of services from the educational program was "abysmal." She said "it's an exercise for us to get ready for actual IEPs once he goes to school." Caroline described it this way:

CAROLINE: Well this is to prepare the family and get us into the rhythm and understanding of - so we're used to the process. And to get like all of his information and to have a paper trail for when he does transition into preschool so they can see growth and where he's come from in the kind of goals we've had, and the kind of kid he's been so that we have something like-it's just really nice heavy governmental paper trail for school.

Summary of parent experiences with EI/ECSE. All participants experienced problems with educational EI/ECSE services. While they felt individuals in the field of education often had their heart in the right place, there was too little funding to provide 
any meaningful support or help. While they were largely dissatisfied participants continued with services because they felt it would have benefits later on when their children transitioned to kindergarten.

Support navigating services: Social media and Facebook. Parents in this study expressed that Facebook and other social media sites administered by and for parents of children with autism were the best resources during their journey to an autism diagnosis and treatment. The parents reported that the social media connections were of greater support and value than large autism advocacy organizations like Autism Speaks or the information by providers upon diagnosis. Karen said, “....as soon as you get that diagnosis, even the preliminary one...reach out to these people because they're the ones who helped me. The other moms. The doctors weren't the ones helping me with that."

At the universal member check meeting we revisited this essential theme. Karen explained how parents before her forged the trail and offered her encouragement.

KAREN: And what's nice now is parents of older kids, maybe not even adults on the spectrum, but teens coming back and going like, 'Just want to tell all you newto-diagnosis moms, younger kids, and whatever, those days were relentless and it was really hard, but it really does get better.; They would talk about where their kid was and where their kid is now.

Every parent in the study used Facebook groups frequently both to seek advice and to offer it. Karen, now an organizer for parent support groups, helped Caroline navigate the system when Nathan was first diagnosed. At the universal member check meeting. Caroline said "you were six months ahead of me and I remember you saying 'wow. I'm so sorry. It sucks. It's not fair. You're kid is still wonderful.' You said all the things I needed to hear.” 
Jessica told me that the autistic mom's Facebook group has helped her with navigating county, medical, and EI services. Social media groups were a guidepost early on in the process

JESSICA: “.. a lot of questions that actually come up for the newbie moms, 'where do I get this type of service and this type of education,'... there is an open communication...it helps a lot and that's the only reason why I'm on Facebook....and...you've got these other people saying 'go here, go here. And push for your child if your mother's instinct tells you to, the parent, or whoever the caregiver is, telling you if something is off. Don't give up. Just go for your child anyway for the different services."

All of the parents in this study learned to navigate services largely on their own,

particularly at the beginning when their child was first exhibiting signs of developmental delays. During the universal member check group meeting, we revisited the experiences the parents had with social media support compared to that of medical providers. The participants engaged in a brief conversation about information needs upon diagnosis. The conversation began when I asked if the parents ever went to autism advocacy websites like Autism Speaks:

KAREN: I never did. I could remember the day they diagnosed [Carter] and [the doctor] gave me this big packet and one thing ...you need to go to Autism Speaks and look at ...the 100-day diagnosis toolkit and these are all the things you have to get done within 100 days. I never even went [to their website] and read it... I was like ...no way.

EMILY: No. I know. it was like a grieving day. I don't even know what to do. KAREN. See so I'm glad I didn't do it looking back because it would have made me feel horrible and I don't think it would have made me feel--

CAROLINE. See that's funny. I looked. I read [and did] everything they told me to do.

KAREN: Did you?

CAROLINE: I did it all... the 100-day thing. It was completely useless.

[Everyone laughs]

CAROLINE: I knew half of it already--[but] what was valuable about it was that it explained different kind of therapies you could use....but that was silly because they give you like eight different-- 
EMILY: They have to give you what your insurance covers.

KAREN:-- which is nothing.

CAROLINE: For us ABA and speech and that's it [all insurance would cover]. KAREN: or only 30 minutes for us.

CAROLINE: The helpful stuff like insurance stuff was never touched upon. Other than, talk to your insurance about specialty services.

EMILY: but no one tells how it's going to be or what it's going to look like or how much-- are you going to go weekly?-- or like my son goes 6 days a week. or how much it's going to cost or how's that going change your life because it changes your life in so many ways.

Summary. Participants' experiences with early services for their children seemed overwhelming to me. During my data analysis I realized how much of the study's interview data focused on talking about service experiences. I reflected on the ways in which the time commitment, frustration, and stress associated with inadequate services may negatively influence life and family experiences. I also realized how vital social media and connections were to the participants.

\section{Essential theme 4: Overcoming obstacles and challenges. All participants} overcame emotional and physical obstacles and challenges. This experience emerged as an essential theme. I spent some time reflecting on the data and parceling out the differences between simply navigating services and overcoming obstacles. I came to see that navigation required finding one's way and blazing a trail. Overcoming emotional and physical obstacles emerged as a separate, discrete lived experiences It was navigation under emotional duress or physical stress. It is while on this trail, navigating and working with providers that parents of young children with autism are hit by unexpected crises and hardship. The participants all described events that were painful, exhausting, and overwhelming. These obstacles were unforeseen and included a child's health crisis, exorbitant unexpected charges for special services, losing or risk of losing critical 
services, and parental trauma. Caroline told me it's harder being a parent of a young child with autism than of a parent of a neurotypical child. She said it's not the autism, “[parenting Nathan is] equally if not more rewarding. You know it's just—it's not hard because of his autism. It's hard because of everything else we have to navigate due to it." This essential theme is unique to this phenomena and touches on the intersecting facets of parenting a young child with autism and navigating special education systems. This section is broken into two subsections (a) impact of service problems on child's development, and (b) emotional impact and grief.

Impact of service problems on child's development. Parents faced hardship having a very young child and navigating early intervention services systems. All participants felt stress and frustration knowing their child needed services and the difficulty they had in accessing them. Emily told me, "they push for early intervention" but then make parents "jump through the hoops before you get all the early intervention stuff. Karen felt similarly, "it's frustrating as a parent to be told get help early. ...even by the doctors 'get help early is super important. Your kid's only neuroplastic for so long.' [Karen felt] 'Great, I'm on your waitlist, is that what getting help looks like? Because that's the most you can give me?" Jessica said "you can't get the time back."

Jessica described her experiences with hardship associated with the phenomena.

JESSICA: [while] having a child with autism is obviously hard...watching your child not develop properly is really hard as a parent...but jumping through loopholes is stressful...a lot of people deal with financial stress....If you look on Facebook groups the price tag of ABA...parents just go without...which is really sad.

Emily told me that she and her husband restructured their lives over the fear of 
losing ABA services. Because of the risk of losing ABA, Emily had to work nights and now isn't around when the kids are at home. She said "this is a two year plan. I just have to remind myself that this is a short-term thing and do something better later on." Service issues caused Caroline stress and frustration, but she also worried about Nathan's health and how that impacted his quality of life. "We had a really rough year last year because he can't tell us and we're constantly taking blood and we're constantly going to the ER and he's throwing up blood and um like that— that expression on his face is like [the expression I wear on my heart]."

Towards the end of the study Caroline shared that Nathan had been suddenly dropped from his $\mathrm{ABA}$ program for logistical reasons, "the very small company took too many kids." Caroline described it as heartbreaking for Nathan and herself. She also observed the impact of the company's business needs on Nathan's development. She said "there was an opening at the educational service district for a four-day intensive ECSE classroom. He regressed socially at his daycare and wouldn't participate in social activities [the way he used to]."

\section{Emotional impact and grief.}

Emotional impact. All of the parents reported stress, frustration, or exhaustion as a consequence of having a child with autism. At times it was knowing your child is not going to develop "properly" or "normally" and what that might mean for their future. However, all four parents reported that the stress, frustration, and grief were less about having an "autistic" child and more stigmatization and lack of support. Parents also cited frustration over ignorance about autism. All of the parents had moments of physical 
exhaustion near breakdown. Karen said the lack of sleep has "led to health issues...I can't remember things from day to day." Jessica experienced stress and worry over not receiving services to help Helen. When Helen was finally diagnosed with autism Jessica said "I was actually crying at the office...thanking the pediatrician for seeing what I see..."

Caroline explained how exhausting it can be to raise a child with autism. She told me:

CAROLINE: ...our family suffers not because he's autistic but because... of the way the world is built and the lack of supports we have and ... just dealing with it all. That speaks to me of a battle ... I'm exhausted, I'm so exhausted from the fight...

Emily felt that while it's hard to have a child who is not developing typically, the biggest stressors are the obstacles to services, particularly cost for those services like ABA that are not covered by medical insurance or available through the educational programs. When I asked Emily if she felt the stress of parenting a child with autism came from the impact of autism or from the service problems she said:

EMILY: It's both. Having a kid with autism is hard, obviously. Watching your kid not develop properly is really, really hard as a parent, but the loophole, the jumping through, the stressors and the financial stress [are the biggest stressors]. Karen's early days of motherhood were marked by exhaustion, isolation, and depression.

She related her early memories of motherhood:

KAREN: Yeah. I would just start breaking down to tears because it is so isolating and so hard...I remember one of those times at WINCO looking down at [Carter in the baby Bjorn] and he looked up at me and it was like we made eye contact and I remember it was like a moment of recognition again. Like he recognized me again and I just remember being like 'he's still in there like he's still in there.' ...It was hard. I mean it's embarrassing, but I only got help for my depression like this year. 
Grief. Towards the end of the interviews I realized I had not explored the idea of grief with the families. I realized that I had not explored it because it hadn't come up as an essential theme. No participant expressed grief that their child had autism or the loss of hope for a typically developing child. However, grief is a trend in the disability literature in general and autism literature in particular (Hayes \& Watson, 2013) and I wondered during my bracketing reflection if this omission was the result of the parents' lived experiences (i.e., not involving grief) or because of my bias towards a social constructivist view of autism. I took the opportunity to discuss this with all four participants during the universal member check meeting. The participants shared common experiences in response to my question about grief and parenting a very young child with autism receiving early services. All parents expressed that the diagnosis came with a strong emotional response. However, since the parents had suspected autism years before diagnosis they were not surprised. Still, the diagnosis had emotional significance for them.

For Jessica the future is unknown because of how Helen's autism may impact her ability to be independent later in life. She told me "[it's the] the ASD piece that never goes away. You have to live with it every day." Jessica told me her husband says "Helen has her own demons and at some point will have to figure it out."

Emily also cited awareness of this possible outcome:

EMILY: Because you always hear all these things like 'my sixteen year old is still in diapers' or 'my kid is never moving out.' Or 'I have to have a little small house next to my house so my kid can have a place to live.' And it's like 'well we'll see how that works out.' 
Towards the end of our universal member check meeting I asked "is there an element of just grieving over your child having autism?" The participants shared their initial responses to receiving an autism diagnosis.

EMILY: I think there is [grief over autism] --to an extent.

CAROLINE: I think it's different [than what the literature says].

EMILY: and I think honestly if the resources were there... and there was service-KAREN: --and you didn't just get a diagnosis and sent home to read a 100 pages.

EMILY: or you didn't have to fight to get there and then get there and now what?

Caroline expressed that she wouldn't have had a sense of grief over Nathan's diagnosis "if you saw other autistic children engaging and being happy and parents who felt like they [their child had a] future [a diagnosis wouldn't have been so hard]." Karen agreed and elaborated on the stigma associated with autism. She described her feelings during the early doctor's visits before the autism diagnosis, "it sounds really bad but when the doctors doubted autism, I would get my hopes up that he wasn't autistic because...[to me] autism was like [being] institutionalized and Rain Man." She said the sense of grief would have been "totally different" if she had seen other autistic children being happy.

Karen struggled and continues to struggle with judgment from the research community and from society in general. She said that no matter what "crazy stuff" comes out of the research, "it's almost always blaming the mother...not even blaming the parents--it's blaming the mom. "Ironically, as Caroline was setting up a game on her phone to preoccupy her youngest son whom she had brought with her to the universal member check meeting, a post appeared on the phone screen. She looked up at the group and laughed. She told us, "I open my phone and what comes up? A post that says screen 
time delays speech."

Karen said she experiences grief in those moments when she "accepts [the judgment and blame] super momentarily." She said she snaps out of it pretty quickly because she knows things like "everybody has to take Tylenol." She said she wasn't sure if it was grief, but sometimes she asks herself, "would my kid have been someone different if I didn't take Tylenol or if I didn't give them screen time or you know that kind of stuff?" But she told the group, "it goes from grief to like 'are you serious? That's ridiculous."

Emily said that she feels fine until out of the blue someone judges her. She shared an experience about a co-worker confronting her saying, "Oh you know, I don't believe in autism I think like bad behavior and bad parenting. 'Karen felt that when Carter was little and younger it was easier to ignore judgment or stigmatization.. She could "play it off' when someone commented about Carter's language delay or social differences. Now because Carter is tall and looks more like a 7 year old than a 4 years old, Karen says now he's so "clearly different than his peers." She said it's "emotional and physical exhaustion...I'm too tired to feel sad [about judgment] any more...I definitely am exhausted and often angry [when] people say stupid stuff. Emily corroborated:

EMILY: ...People say stupid stuff. [For example] 'you gave your kid the 18 month vaccination there's a definite correlation [with autism].' I'm like "you are ignorant." I don't get opinionated until they start talking about autism then I'm like 'I HAVE an opinion.'

Essential theme 5: Ingenuity, resilience, and perseverance. Ingenuity, resilience, and perseverance emerged as strands in an essential theme in the phenomenon parenting a young child with autism and navigating the service systems. All four mothers 
were ingenious not only with piecemealing a service plan for their child but also in the accommodations and adaptations to support their child's successful engagement and participation in typical childhood experiences.

Accommodations. The way parents made accommodations for their autistic children was one of the most salient themes in this study. Almost as soon as parents noticed differences in their children's development, they began making changes in their environment to support engagement and emotional regulation. This was part of their role as parent. Caroline described it this way, "I don't want to say we bend over backwards, but we really do a lot to accommodate him.... and yet it's so normal [for us to adjust our lives] like he's on the potty in the kitchen." Caroline shared "we throw the rules out the window." Jessica also expressed that daily life requires accommodations. She told me that even going on a day trip, " you have to think of what will be there....even though you don't know what's going on there. Like what would be trigger point for her?"

All of the parents in this study expressed an awareness that their children needed to successfully engage in a neurotypical world in order to have the same opportunities as peers. Jessica told me "... [we] just want her to have as much experience as possible like neurotypical kids even though ... certain things we can't provide but we want to try. I mean if it fails like oh well..." Jessica said in order for the neurotypical world to be accessible, “...you have to strategically plan [every activity]...”

Table 5 provides examples of accommodations parents made to support their children's participation in everyday childhood activities and experiences. 
Table 5

Participant Activities to Support Child Engagement in Typical Childhood Activities

Participant

Description

\begin{abstract}
Caroline [Supporting Nathan in preschool social and play routines]...this to me is the quintessential [example]...he's a community member. He is an important member of this [preschool] community — and all the kids [are] ...but he needs help. He needs support to engage in this community-

Emily [Through fostering Joshua's relationship with extended family] ...It's just very telling of how, how good she is with my children and how much they love her."

Jessica [We set up a communication board]...[we] figured out... the problem, and we kept practicing and practicing, and now she can say, ' $I$ ' $m$ thirsty, I'm hungry, ' and now it's at the point where she can get rid of this program board and she can just say [what she wants or needs."

Karen [Wearing sunglasses...] ...you don't know if he's making eye contact or not... Because he's still paying attention to you and still interacting but when he's confronted with some heavy eye contact, it will shut him down...So he's able to divert his eyes and still behave... socially. Yeah. He kind of... It's part of his armor. It's part of his uniform.
\end{abstract}

Adaptations for success. All of the parents in this study described helping their children successfully engage in typical childhood activities. This required addressing contextual issues that impeded participation. Karen ameliorated some of the effects of Carter's sensory issues through providing headphones, sunglasses, gloves. Karen told me about playing with Carter in a recent snowstorm something he had been unable to do until recently because of his overwhelming sensory issues, "We went out and played in the snow and we walked all around. He wasn't afraid... He would walk through the snow and lead us... And it was really great because before... everything sensory [overwhelmed 
him]...”

In order for Helen to participate in the same classes and programs as her peers with typical development, Jessica and her husband put in hours of research and planning for adaptations. Jessica said "It's not like we [just] sign up for preschool swimming class." She told me she had to find a specific class that "knows special needs population [and]...feel confident in what they're doing." Fortunately, her local recreation department offered an inclusion program otherwise, they would "have to look into private lessons and...pay significant amount to provide something like remotely close to what the other kids her age are doing."

Emily and her husband fostered close relationships with immediate and extended family members. As a result Joshua participated in the family life in the same way as his siblings. Emily's family used social media to foster relationships between close family members and their children. She described her sister's relationship with her children, "She sends presents and calls them on the phone and talks to them. They like to Snapchat her...send funny pictures of themselves and she Snapchats [them]."

Caroline felt an important aspect of adapting environments for Nathan is to maintain open communication with the communities he is a part of. She told me her perspective is, "My kid is autistic and come talk to me about it and ask me whatever question and don't you dare feel bad about asking..." Caroline felt this not only benefited Nathan but also the community of parents, "[Nathan] is their experience with autism and they're getting it early and they're getting it every day--they're seeing it every day."

Essential theme 6: Reflecting on the first three years and looking forward. By 
the end of this study, all four participants were preparing for their children's entry into kindergarten. They had made it through the journey from early recognition of autism symptoms to securing services. Emily described her learning curve, “...a lot of times parents of kids with autism...learn to stand up for themselves because they're like 'my kid needs this service you need to offer this service to them... '”

For Karen reflection included seeing how far they've come and Carter's increasing enjoyment in life and engagement with the world, "he's [becoming] more able to enjoy something without a bunch of accommodations." Emily's comments reinforced Karen's. Emily's family is at the end of the journey from early concerns to autism services. She's noticed how far her extended family have come.

EMILY: It's funny. They're like, 'oh I talk about my grandson with Autism all the time.... ' I'm like, 'Okay, [denied it] before, just rolled your eyes'. [Now] my dad is like, 'see, he made eye contact with me.' He'll be on the webcam and say, 'See, look, I saw his eyes, he made eye contact.'

Jessica described a sense of accomplishment over how far her family has come through her perseverance. Participation in this research study helped her recognize and articulate this experience.

JESSICA: Yes, it's kind of like nostalgic. I was thinking like this and pushing so much, and now we still have so many unknowns going into kindergarten. The things I feel accomplished of, how much we came.

RESEARCHER: By looking at that and thinking about that?

JESSICA: Yes, and even how much she grew, that Hannah grew. It's nice to reflect. If I don't have this opportunity I don't reflect.

At the universal member check meeting Karen told the group that in participating in this study made her realize, "we've come so far." She said, "I was realizing it along with you (i.e., me the researcher) as we went through these photographs. Like 'oh wow, 
we really have come so far.. I don't think you feel it in your day-to-day life. just like you don't really see your kid get older, day to day..." Emily added "it would be good to follow people's paths and see where the interventions take them and where the school system takes them. I think that would be fantastic."

\section{Limitations}

Two methodological limitations associated with my study will be discussed in this section: (a) validity and (b) bias. Issues of validity are common in qualitative research. Research validity is an experimental construct more aligned with the aims quantitative research. Qualitative researchers are less concerned with generalizability than a deep understanding of participants' experiences.

\section{Validity}

Lincoln and Guba (1985) address issues of validity in qualitative research and outline methodological approaches to reduce this paradigm weakness. Further, Colazzi (1978) addresses validity problems specific to phenomenological research. I addressed research validity on several levels consistent with both models. I employed three levels of member checking and confirmed concordance of essential themes with participants. I also debriefed and reviewed themes with a phenomenological expert. In addition I triangulated data. I followed Colazzi’s method which "emphasizes any new data that emerges from [participant member checking] must be worked into the final product of the research.” (Colaizzi, 1978, p. 62), I applied rigor and adherence to my proposed research procedures.

Despite these activities I recognize that issues of validity remain, 
particularly from the perspective of phenomenological research philosophy, which is concerned with identifying the universal structure of a phenomenon under investigation. For example, Gorgi (2009) questions the usefulness of member checking with participants to address validity in phenomenological research noting, "participants are surely privileged when it comes to what they experienced, but not necessarily concerning the meaning of their experience." This view holds that phenomenologists are the experts and finding the essential structure involves a deep phenomenological analysis. If participants can describe or confirm the meaning, Gorgi argues, then why would the phenomenological researcher "go through such a long procedure and possibly not get it right? (Giorgi, 2012 p. 358 ).” Nonetheless I chose to employ Colazzi's methods to address validity with recognition of the limitations. I made this choice because the aim of my study was twofold: to describe the essential themes and to accurately represent participants' voices.

\section{Bias}

Descriptive phenomenology involves bracketing of researcher and participants' preconceptions and biases about the phenomena under investigation. From an idealist framework, "bracketing is characterized by the near-total exclusion of all internal and external suppositions" (Gearing, 2004, p. 1436). I engaged in bracketing strategies using methodological procedures described in the phenomenological literature (Vagle, 2014). Bracketing in descriptive phenomenological research is akin to mathematical bracketing where by some operations are solved first while others are temporarily suspended (Gearing, 2004). The activities I engaged in as a researcher addressed bias on a personal 
and external level. I incorporated activities into every phase of the study in order to "bracket" out preconceptions, biases, and suppositions (Vagle, 2014). Early on I researched and reflected on conflicting theories on autism research and intervention. This served to create a neutral stance through which I could begin my investigation.

Throughout the interview and data collection phases I used a bracketing journal, member checking, and dialogue with participants to challenge biases or interpretations. I debriefed with a phenomenological expert about the data and emerging themes. A final step in the bracketing process is to re-introduce the concepts that have been bracketed out. I found success in employing these strategies and believe they were effective in reducing bias and isolating the phenomenon and identifying essential themes. However, I recognize that the findings reported in this chapter have likely been influenced by biases to some degree despite my attempts to neutralize them.

\section{Chapter 4 Summary}

Reporting on phenomenological data has been described as an ethical endeavor. The goal is to represent the participants' lived experiences without distorting findings and the meaning from the participant' perspectives (Plummber, 1999). The primary aim of descriptive phenomenology is to describe rather than explain (Hurssel, 1970). As a result, I engaged in ongoing bracketing activities and returned often to the participants and data. While Caroline, Emily, Jessica, and Karen were a homogeneous sample in many ways, particularly having the resources and education to navigate the early intervention systems for their children with autism, they were also distinctly different in their lived experiences. Caroline herself was autistic. Emily had three children and married at a 
young age. Karen struggled with a traumatic birth and postpartum depression and Jessica's native language was not English. Certainly these heterogeneities held stories of their relationship with the phenomenon. However, consistent with phenomenological philosophy, because they were in contact with the same phenomenon, essences rose to the surface.

My study's findings have implications for changes in practice. Most notably essential themes from this study indicated a need for improved screening practices and service delivery. Given that early screening protocols and tools are in place, my study's findings suggested a need for improvement in provider approaches to working with families with concern for their child's development. Secondly, participants' in this study reported negative experiences with service systems. There was no coordination, access was difficult, and resources were lacking.

In sum, the lived experiences of the four participants caring for a very young child with autism and navigating the early intervention services systems provided insight into the phenomenon. The essential themes offer insight and may serve as lessons to individuals in professions who support families in autism early intervention. Consistent with the metaphor Blazing a Trail, the findings of this research may help other parents navigate early intervention systems as they begin to recognize emergent manifestations of autism in their children. Chapter 5 presents my conclusions and future directions for research in the field based on the study's findings. 


\section{CHAPTER 5 \\ REFLECTIONS, CONCLUSIONS, AND LIMITATIONS}

\section{Overview}

The greatest aim of this study was to give voice to parents of very young children with autism. This study addressed the research questions in the participants' own words. While many research methodologies shed light on the experiences of young children with autism and their families, there is a great deal of value in hearing parents describe how they first came to suspect their child had developmental problems and the subsequent experiences that eventually led to diagnosis and services. While the findings of this study cannot be generalized to all parents of children with autism (and that was not the goal), it allows an accessible view into the participant's lives. Descriptive phenomenology captured the type of data rarely presented in the literature. Through their own voices, participants invited other parents, providers, and researchers into their world. In an area of research dominated by empirical investigation and structured intervention, the findings of this study present a new perspective on parenting very young children with autism. Sokolowski (2000) wrote "[phenomenology is] a way of looking at what we usually look through" (p. 12). Phenomenology as a philosophical movement was in direct response to empirically bound ideas about the meaning of the human experience and the world. Instead of investigating objective agreed-upon constructs in the lives of participants, my study isolated truth as it is experienced by participants and elucidated the phenomena through emergent essential themes. Chapter five situates the findings of this study in the research literature and explores the implications and future directions of the results presented in chapter four. 
This chapter will be organized in the following sections (a) an introduction to findings, (b) a synthesis of the findings and discussion of how my study addressed the research questions, (c) the implications of the findings and future directions for research, and (d) a summary.

\section{Introduction to Chapter 5}

\section{Literature Review and Theoretical Frameworks}

Literature review. The aim of the study was to investigate participants' lived experiences parenting their very young child with autism. The study also sought to gain insight into parents' perspectives about early intervention services. This aim was particularly important because the field of autism early intervention (AUT-EI) is an emergent area of study and practice (Camarata, 2014; Zwaigenbaum, 2015) and few qualitative studies have investigated parenting and this context (Grindle, Kovshoff, Hastings, \& Remington, 2009). The literature review in Chapter 2 distilled the current AUT-EI research into four elemental themes that emerged from the literature review: (a) early intervention and autism; (b) caring for an infant with autism; (c) autism and the Individuals with Disabilities Education Act, Part C (IDEA, Part C); and (d) parent perspectives. These themes intersected and, as a whole, represented the most salient research findings about parents with a very young child with autism

Perhaps the greatest correlate between the literature review and my study was the emergent state of AUT-EI. At the time of the literature review first generation AUT-EI models were still in the preliminary research stage. Participants in my study reported service providers lacked awareness and understanding of infant / toddler manifestations 
of autism. This finding suggested that there is a research to practice gap. The participants in this also study reported a dearth of autism-specific services when their children were under age three. My study's findings illuminated the nuanced parenting experiences in the absence of clinical expertise and services for infants and toddlers with early manifestations of autism.

Qualitative studies of older children with autism examined and reported on determinants of service satisfaction and the effects of child autism on parenting experiences (Cocoran, Berry, \& Hill, 2015; DePape \& Lindsay, 2015). However, only a handful investigated lived experiences in these contexts (Osborne \& Reed, 2008). Lived experiences have a specific denotation in phenomenological research. Lived experiences are the meaning participants make of everyday practices "in such a way that they are not destroyed, distorted, decontextualized, trivialized or sentimentalized" (Benner, 1985, p. 6). The focus of this methodological paradigm is the subjective experience with an examination of participants' detailed lived experience as a way of making sense of the world.

The literature review yielded no studies employing a phenomenological method to explore parenting and services for children with autism between the ages of birth and three. Given that this field of research is largely unchartered, a phenomenological analysis was an appropriate choice. The results of this study may contribute to hypothesis development about supporting parents of very young children with autism. Hypothesis development is a well established aim of qualitative research (Cresswell, 2005).

The participants' described their journeys through prompted conversations with 
me about family photographs. Narrative and photographic data generated rich and deep descriptions of the participants' stories. The photographs offered an opportunity for participants to reflect and make meaning of their world in a way that traditional interviewing methods alone would not have (Clark-Ibáñez, 2004; Harper, 2000). ClarkIbanez (2004) noted that using photographs with interviews may trigger meaning that would otherwise have been missed. In my study, photographs acted as a bridge between my lived experience as the researcher and the parent's lived experiences related to the phenomenon. As we talked about the images and memories of family life I felt less like a researcher and more like another parent talking about our kids. While the prompts and research questions guided and structured the interview process, the dialogic photographic interview methodology neutralized the researcher / participant dynamic.

Essential themes and their meanings rose to the surface of the data through conversations with the participants, data analysis and reflection, and member checking activities. Through looping and returning to the data repeatedly, these essential themes illuminated the structure of the phenomenon and a discernable model recognizable by all participants. I came to see that the essential themes had a temporal association and reflected the three-year journey from the time of first concerns to autism intervention. While I found no AUT-EI phenomenological studies in the literature review, the lived experiences of participants in my study correlated with trends in the emergent s AUT-EI literature. Parallels with current research included (a) parental recognition and descriptions of early manifestations of autism and (b) reported lack of available services for their very young children with autism. My findings were consistent with Stahmer and 
Mandell's (2007) findings that EI services across 50 states were available for only a small percentage of infants and toddlers later diagnosed with autism. None of the children with autism receiving EI received specialized interventions to address emergent autism

Theoretical frameworks. In this chapter I explore and describe how this study addressed the two research questions. This exploration was informed by the two theoretical frameworks (a) descriptive phenomenology (Husserl, 1979) and (b) Ecological Systems Theory (Bronfenbrenner, 1979). Given the methodological approach and the theoretical lenses, I hypothesized that the results would reveal the contextualized lived experiences and the effects of bidirectional ecological influences on parenting a very young child with autism (Bronfenbrenner, 1979; Dunst \& Trivette, 2009). While each participant experienced the phenomena in unique and meaningful ways, essential themes emerged across all participants, illuminating the way in which the research questions were addressed.

\section{The Research Questions}

This research study sought to answer the following research question and two subsidiary questions:

(1.) What are the participants' lived experiences as parents with a young child (under age five) with ASD as they relate to early intervention services?

(1a.) What are the participants' experiences of having a young child diagnosed with ASD within the past two years?

(1b.) What are the structural essences of the phenomena of parenting a very young child with ASD in the context of EI services?

\section{Lived Experiences and Essential Themes}

Participants' lived experiences. As I spoke with parents it became clear that while lived experiences addressed the two research questions, there wasn't a discrete parenting experience and a discrete service navigation experience. This was contrary to 
my expectations as the research question suggests. I had hoped through phenomenology to untangle the association between a child's autism and parenting. I hypothesized parenting would stand out as a construct and in isolating it I would find fundamental qualities universal to all parents, independent of the child's disability status. However, the data showed autism characteristics, autism-specific services, and parenting were woven strands in of interconnected lived experiences. Early into the first interview Caroline told me, "[Autism] is such a huge part of our life. It's so central to everything we do." It is fitting that subsidiary questions (1a.) and (1b.) combined answered the predominant research question, What are the lived experiences of parents with an infant or toddler with autism as they relate to early intervention services?

Essential themes and subthemes. Each participant's lived experience revealed the multi-directional intersection of parent, child, and social context. Disability rights advocates often describe disability as "socially constructed" and therefore one's way of being as defined by arbitrary, often disempowering views of ability (ASAN, 2009; Prizant, 2015; Robertson, 2010). The phenomenological research lens allowed that philosophical concept to surface cohesively with the essential themes. The Ecological Systems theoretical lens explained the mechanisms by which ecologies of support influenced participants' capacity to enhance their child's development. Together these theoretical frameworks guided my literature review and the pursuit of the research questions.

The results presented in this chapter report on the participants' lived experiences and my engagement with the data collection and analysis. The findings reflect the way 
parents made meaning of their lived experience and the essential themes comprising the phenomena of parenting a young child with autism receiving early intervention services.

Table 6 summarizes the essential themes and subsidiary themes that emerged from my phenomenological data collection and analysis.

Table 6

Essential Themes and Subsidiary Themes

Theme

Subtheme

Pioneering

Making the journey as a family

Navigating the undeveloped (new world) of autism EI service systems

Overcoming obstacles and challenges

Ingenuity, resilience, and perseverance

Reflecting on the first three years and looking forward
- Parent recognition of early markers

- Invalidation from others

- Forging a trail for future families

- Belonging and immediate family

- Bridging the relationship with autistic children and extended family members

- Intersecting service systems

- Pediatricians / medical providers

- Educational services

- Support navigating: social media and Facebook

- Service breakdowns

- Impact of autism service issues on child

- Emotional impact and grief

- Accommodations to address the effects of autism on everyday life

- Adaptations to childhood activities

- Looking back on the journey so far

- Preparing for kindergarten 


\section{Section 1: How My Study Results Addressed the Research Questions}

\section{Overview}

This section will discuss the findings of the research study and how they addressed the two subsidiary research questions. First I will discuss findings that answered Subsidiary question 1a. What are parents' experiences of having a young child recently diagnosed with autism? This subsection described the individual lived experiences related to a recent autism diagnosis and the reported experiences and parent perspectives. The second subsection presents the study's essential themes and answers subsidiary question 1a. What are the structural essences of the phenomena of parenting a very young child with autism in the context of EI services?

\section{Research Question (1a): What are Parents' Experiences of Having Young Child Recently Diagnosed with Autism?}

The findings in this study revealed that participants' lived experiences with their young child recently diagnosed with autism affected all aspects of parenting. Participants sought answers as soon as they recognized a problem in their children's development. Several of the participants stated they had a sense from birth that their child had developmental issues. All parents had concerns by the time their children turned nine months old. Parental lived experiences with a young child with autism clustered around recognizing symptoms, seeking a diagnosis, and navigating early service provider responses.

\section{Recognition of early markers and facing invalidation from pediatricians.}

Early recognition of markers and invalidation were two of the earliest lived experiences 
reported by all participants. Participants had concerns at two points early in their child's development. First parents reported general concerns about their child's development to their pediatricians at 9 months on average. Parents began having and reporting concerns about autism specifically at 19 months on average. This age of recognition finding is consistent with the research in the field. It is established in the literature that parents noticed symptoms of autism between 18 and 24 months (Baghdadli et al., 2003; Charwaska et al., 2007; DiGiacomo \& Fombone, 1998; Goin \& Myers, 2004; Ozonoff et al., 2009).

Infant markers are also well established in the research literature. The literature review in Chapter 2 described manifestations of autism in infants and toddlers. Although there is a range of emergent manifestations, two of the most marked emergent behaviors are differences in social communication (e.g., orienting to name, imitation, affect, gesture use, social smiling, social interest) and sensory processing problems (Bryson et al., 2007; Ozonoff et al., 2011; Presmanes, Walden, Stone, \& Yoder, 2007; Zwaigenbaum et al., 2005). Parents in my study reported recognizing these differences in their infants early on. Table 7 presents participants' reports of early manifestations of autism in their children before age three.

Table 7

Participants' Report of Their Child's Earliest Manifestations of Autism

Domain Description of Early Autism Symptoms

Language Caroline - He threw himself on the ground....and started crying....something didn't go how he planned and he had no way to communicate it.

- He only turned to look at you about half the time 


\begin{tabular}{|c|c|c|}
\hline & & when you called his name. \\
\hline & Emily & $\begin{array}{l}\text { - He flunked his newborn [hearing] screening twice. } \\
\text { - } \text { [At } 30 \text { months] he didn't have any verbal skills at } \\
\text { all. } \\
\text { - He would pick up a couple of things and then he } \\
\text { would lose them. And then he would pick up a } \\
\text { couple more things and then he would lose } \\
\text { them...he just kept having that cycle }\end{array}$ \\
\hline & Akikio & $\begin{array}{l}\text { - We started...speech therapy by age } 20 \text { months or } 18 \\
\text { month-ish. And then they said 'yes there is language } \\
\text { delay and then something about the social interaction } \\
\text { but then they didn't think it was autism }\end{array}$ \\
\hline & Karen & $\begin{array}{l}\text { - He didn't talk a lot before [the regression]...he had } \\
\text { like ma ma, da da, ba ba, kitty, baby. it was pretty } \\
\text { much like that... He vocalized a lot... just a lot of } \\
\text { exploring sounds...--He lost speech...He didn't have } \\
\text { a ton of speech [but he lost it]. }\end{array}$ \\
\hline $\begin{array}{l}\text { Social - } \\
\text { Communication }\end{array}$ & Caroline & $\begin{array}{l}\text { - He comes in and out of engagement. We had to } \\
\text { learn how to engage him, and interact with him, } \\
\text { and how to get his attention and how to talk to him. }\end{array}$ \\
\hline & & $\begin{array}{l}\text {-...Before his autism diagnosis he qualified for [EI } \\
\text { for] social and communication. He was like a } \\
\text { negative } 12 \text { out of } 100 . . \text { he's severely deficient. You } \\
\text { have to be } 2 \text { standard deviations below. }\end{array}$ \\
\hline & Jessica & $\begin{array}{l}\text { - He was losing skills and I was like 'that was really } \\
\text { weird' and he would do something really cute or really } \\
\text { smart and I could never get him to repeat it. }\end{array}$ \\
\hline & Karen & $\begin{array}{l}\text { - The M-CHAT...shows that [a] child doesn't respond to } \\
\text { his name, like eye contact and all of that. It was 50/50 } \\
\text { percent [with Helen] so it was so hard to see, and so } \\
\text { that's why I was bringing her to the pediatrician. Half } \\
\text { of the time she does, half of the time she doesn't. } \\
\text { - She [would] not really look... at the camera, she was in } \\
\text { her own world. }\end{array}$ \\
\hline
\end{tabular}




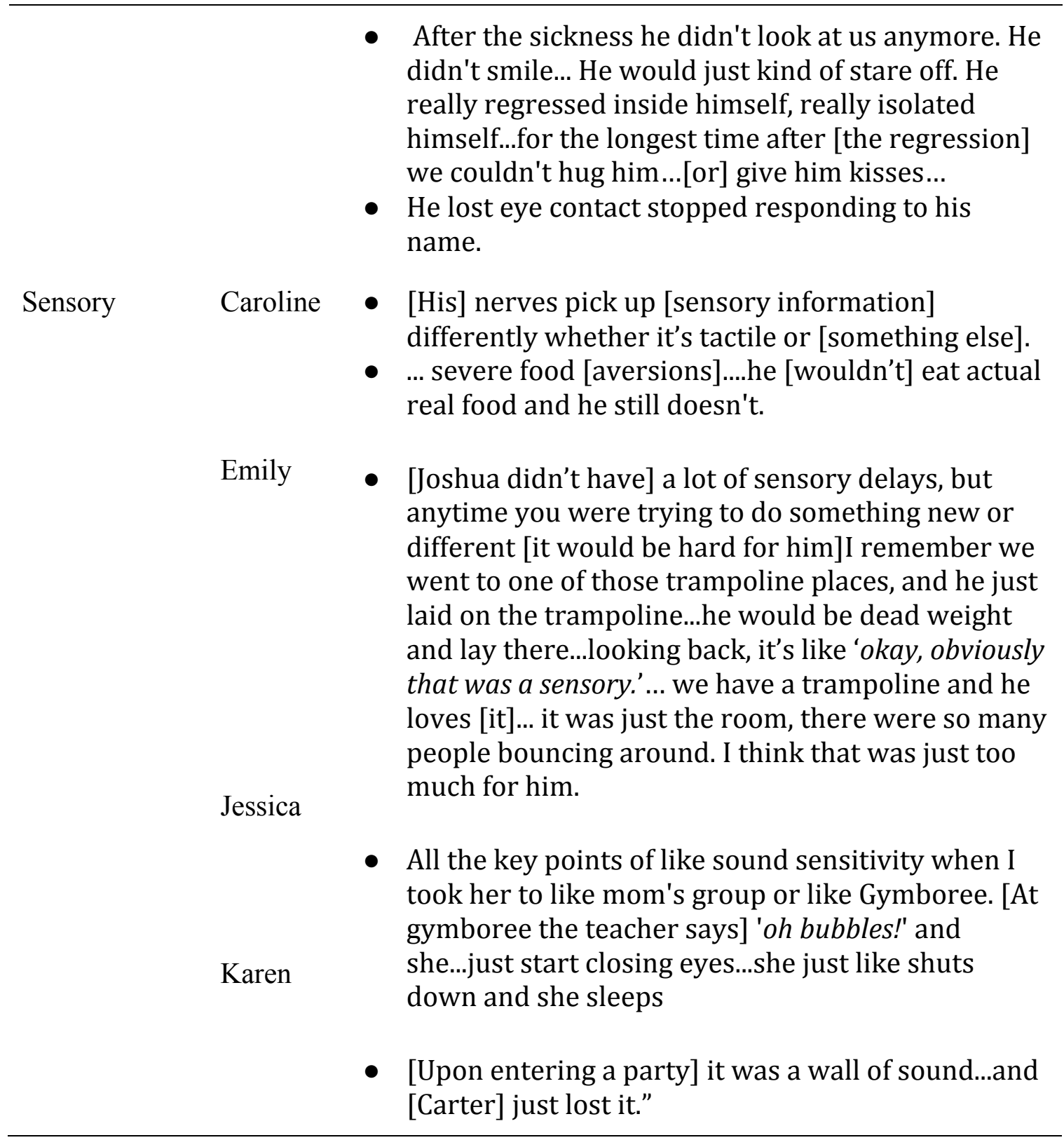

The robustness of this sub-theme suggested that while parents recognized infant markers of autism, providers did not. This finding contrasted with one of the few reviews of qualitative investigations of parents' experiences during the diagnosis phase. DePape and Lindsay (2015) found that parents needed information from service providers about early autism in order to help parents recognize ASD markers in their infants. My study 
found the opposite trend: parents were helping providers recognize ASD markers in their infants and they continued to do for an average of 22.5 months from first expressing concerns to finally receiving an autism diagnosis.

The literature review also described a lag time between age of parental concern for their child's development and commencement of autism services. In other words the research literature reported parents recognized developmental problems when their young child was 18 months on average (Rogers, 2009) and most children with autism are not diagnosed until they over age three (Baio, 2012; Elsabbagh \& Johnson, 2007).

To address the need for earlier identification of autism in young children, The American Academy of Pediatrics recommends pediatricians screen children at 18 and 24 months during well baby checks (Johnson \& Myers, 2007). These efforts are aimed at identifying infants/toddlers at risk for autism early on in the hopes of earlier intervention. All of the participants in my study reported that pediatricians disregarded failed screenings or dismissed parents' concerns about autism. Jessica and Emily reported that their children were given the MCHAT at their well baby checks and neither child passed. Nonetheless, the pediatricians told them to wait and see. The doctors would not make a referral to a developmental pediatrician for a comprehensive autism evaluation. Jessica and Emily both felt they had answered the MCHAT questions honestly and accurately yet when their children failed the screen the pediatricians expressed that their observations were inaccurate reflections of their children's behavior.

Feelings about accessing services for their child. Consistent with other research in the field (e.g., Brogan \& Knusse, 2003; Goin-Kochel, Mackintosh, \& Meyers, 
2006), participants in this study experienced stress and frustration when accessing and navigating services. Parents in my study described a complicated service system that included medical, educational, insurance providers, private, and county services. The participants described stress and frustration with the lack of resources, coordination, and predictability. These concerns were congruent with findings in the research literature and included: process and speed of diagnosis (Corcoran et al., 2015; Osborne \& Reed, 2008), difficulty accessing services (Brogan \& Knusse, 2003; Goin-Kochel, Mackintosh, \& Meyers, 2006 ), lack of availability of services (Brogan \& Knusse, 2003; Goin-Kochel, Mackintosh, \& Meyers, 2006), and providers minimizing parental complaints and concerns (Applequist \& Bailey, 2000; Bailey, Scarborough, \& Hebbeler, 2003; Hurtubise \& Carpenter, 2011; Jackson, Traub, \& Turnbull, 2008).

My study also generated new findings about stress and frustration associated with service experiences including: invalidation from others about concerns and self doubt (e.g., "I'm just a mom," "I doubted myself."), lack resources and poor educational services (e.g., "It’s abysmal," “A waste of time," “Meaningless.”), medical wait times and service interruptions due to medical system bureaucracy (e.g., "...we called in May...and I was able to get in for the speech evaluation in September..."and "when they called in December, the office told her "we're booked out until May," and parental management of a complex and uncoordinated network of service systems (e.g., "the time I spend--hugely-- is navigating [services]," “[you] play phone tag and research what resources are out there," and "[you have to] keep pushing [providers]."

Navigating services birth to age three. Also consistent with the literature and 
reinforcing the emergent state of AUT-EI (Rogers \& Vismara, 2014 ; Stahmer \& Mandell, 2007) none of the parents received autism-specific EI educational services until their children were over age 3 . The services during the EI years did not specifically address emergent symptoms of autism, a trend across U.S. state special education programs (Stahmer \& Mandell, 2007) The children in this study received IDEA, Part C services for developmental delay or language delay. Participants in this study received inhome parent coaching an average 1.75 hours per month. To compensate for insufficient services through education system programs, parents in this study sought specialized speech and occupational therapy through private medical services. However, often they experienced difficulty obtaining insurance coverage without a formal autism diagnosis. Another more disconcerting finding in my study was that parents' experiences with the IFSP process were out of compliance with IDEA, Part C. These shortfalls included: educational programs offered one size fits all service programs that were not individualized to child or family needs, no specialized AUT-EI models, and services hours well below the state's recommended minimum EI (i.e., birth to three) service dosage of one hour per week (OLIS, 2017). In my study Caroline, Jessica, and Karen received in home EI coaching one hour per month while Emily received one hour per week.

These finding suggests that Oregon's approach to EI for autism may be in need of reform, particularly in the area of resources dedicated to families of young children with autism. Participants in this study worried about the effects of delaying EI services due to provider invalidation, wait lists, or inadequate service options. I found no studies in the 
literature that directly associated delay in diagnosis with parental worry over their children's development. The qualitative studies I reviewed on the topic focused on clinical aims like determining age of onset and early indicators of autism. Most qualitative research I found described experiences with older children. This is likely attributed to the emergent status of AUT-EI research and the focus on quantitative aims of research in the field. However the qualitative studies with older children found parents experienced frustration over length of wait-time between EI referral/assessment and determination of edibility/service delivery (Bailey et al., 2003; Bailey et al., 2004; Grindle et al., 2009; Haring, Lovett \& David, 2001; Shannon, 2004; Wade et al., 2007; Webster et al., 2004). Corcoran and colleagues (2015) found parents expressed frustration with the diagnosis process, lack of service quality, and lack of direction or support from providers. There was also near consensus among dominant researchers in the field of AUT-EI that early treatment is a high priority (Camarata, 2014; Dawson, 2008; Elsabbagh \& Johnson, 2007; Rogers \& Dawson, 2010; Zwaigenbaum et al., 2015) citing missed critical opportunities for greatest impact of intervention effects (i.e., birth to three).

Stress. Parenting stress has been widely reported in the autism literature. However, the literature review for this study yielded few studies investigating ecological factors influencing stress in parents with children with ASD. Osborne, McHugh, Saunders, and Reed (2008) proposed that life conditions likely moderated parental stress (e.g., fatigue, intensity of intervention, and competing priorities associated with family life). Most of the ASD-EI research linked parent stress with infant disability factors (e.g., 
symptom severity or behavioral problems) or parent responses to their child's condition (e.g., grief). Hayes and Watson (2013) conducted a meta-analysis of parent stress studies and reported that parents of children with ASD had much greater overall stress than parents of children who were typically developing and parents of children with developmental delays. This finding suggests that factors associated with having a child with ASD were inherently more stressful.

Participants in this study reported stress and exhaustion but often emphasized it wasn't only the "autism." Rather, they reported that the service system made life stressful. The results from this study challenged the prevailing view that having a child with autism is stressful primarily because of the child's disability. As Caroline described:

CAROLINE: Our family suffers not because he's autistic but because ... of the way the world is built and the lack of supports we have and ... just dealing with it all. That speaks to me of a battle ... I'm exhausted, I'm so exhausted from the fight...

This distinction may be an important one to consider when developing programs to support families with a very young child with autism. Attributing child stress to a child's "pathology" has implications for treatment approaches.

Some studies described grief and other negative emotions over having a child with autism. Cocoran, Berry, and Hill's (2015) reported in their meta-synthesis of 14 studies on lived experiences parenting children with autism:

Caring for a child with ASD is an emotionally taxing experience and parents reported intense negative emotions. From the point of diagnosis, parents spoke about denial, grief, and disappointment. (p. 358)

Similarly, Pape and Lindsay (2015) conducted a metasynthesis of qualitative research on parenting experiences caring for a child with autism and found during the 
initial stages of diagnosis parents felt feelings of guilt and blame, looking for causes and explanations for their child's autism.

In contrast, participants in my study reported they would not have had negative feelings about their children's autism diagnoses if there wasn't such a stigma attached to autism. At the universal member check meeting all participants affirmed Caroline's assertion that "if you saw other autistic children engaging and being happy and parents who felt like they [their child had a] with a future [a diagnosis wouldn't have been so hard]." This finding might have implications for providers. Service outcomes may be mediated by this decontextualized view of parental feelings about their children's autism and possible mental health correlates. The participants responses in my study reinforced an ecological systems view and challenged the medical model paradigm of pathology.

Social media through early concerns and diagnosis phases. The literature review for Chapter 2 yielded few studies examining the role of social media in the lives of parents of very young children with autism. One of the most salient findings in this study was the powerful support role social media played in each participant's life. When asked specifically about informational sites like Autism Speaks none of the participants credited formal websites as a source of support. Instead, parents depended on Facebook autism mom's groups for every type of support imaginable through every phase of their journey. They reported they trusted the other mom's experiences and that night or day they could reach out and there was validation and advice.

JESSICA: You have [professionals] saying 'you just have to wait, don't be anxious...,' and you've got [other parents on Facebook] saying 'go here, go here...push for your child if your mother's instinct tells you to.' [On Facebook it's]...the parent...or caregiver... telling 'you if something is off don't give up. Just 
go for your child anyway... for the different services.'

Participants in this study also reported that now that they are through the diagnosis and EI phase, they help the "newbie" moms who are just starting out. Supports participants reported included:

- Restaurants and businesses that are friendly to kids with sensory issues,

- Specific therapists or teachers who are especially good,

- Who to call for Oregon health Plan coverage,

- Advice on whether to give child medication,

- Whether their children's symptoms are consistent with autism,

- Service information, and

- Information about autism symptoms.

This finding illuminated a possible disconnect between practitioners and parents of young children with autism. While providers disseminated information about autism and treatment, participants reported it as overwhelming, stressful, or useless. Social media resources were largely absent from the research literature likely reflecting the relatively recent prominence of social media and internet information in everyday life. However, Grindle and Colleagues (2009) found parents valued opportunities to establish social networks and relationships with other families of young children with autism. Studies focusing on autism services from pre-diagnosis to services for older children repeatedly found parents desired more information. The social media findings in my study add a nuanced detail about the type of information parents found useful. In a focus group study, Osborne and Reed (2008) reported parents wanted improvement in provider information provision about autism. This included what to expect, diagnostic process, and information about organizations and services. This finding was particularly salient amongst parents with preschool aged children with autism given that $84 \%$ of this group 
reported the need for improved information and resources.

The role of social media in parent access to information in my study has implications for service. Not only do groups like Facebook or parent organized on-line groups provide connection to other parents but in my study they helped parents identify and access services that grew with family needs. Participants in my study sought help when they first had concerns about their child's development and continued to do so as their child became school age. Social media connections provided immediate answers and helped participants navigate the reportedly complicated service systems. Studies found in the literature review reported service navigation, wait times, and lack of information as sources of stress and frustration (Coogle, 2013; Corcoran et al., 2015; Osborne, McHugh, Saunders, \& Reed, 2008).

This finding may be particularly applicable in rural areas or situations where families are otherwise isolated. These groups face challenges in accessing services (Mello, Urbano, Goldman, \& Hodapp, 2016). Further, medical and education programs may benefit from adopting online parent peer support and incorporate components into support resources following an autism diagnosis, a time when parents in my study reported wanting support and easy to understand information.

\section{Research question (1b): What are the structural essences of the phenomena of parenting a very young child with autism in the context of EI services?}

As described in Chapter 4, six essential themes emerged from the data analysis and interpretation. These themes were temporally bound experiences in the three year journey from first concerns to autism treatment for the participants' children with autism. 
The six essential themes are (1) Pioneering, (2) Making the Journey as a Family, (3) Navigating the Undeveloped New World (EI Autism Service Systems), (4) Overcoming Obstacle and Challenges, (5) Ingenuity, Resilience, and Perseverance, and (6) Reflecting on the First Three Years and Looking Forward. Each of the themes marked a milestone for Caroline, Emily, Jessica, and Karen as they navigated the uncharted new world of services for emergent autism. Parenting, autism, and services intertwined and strands of each were present in all six essential themes. This following subsections describe how the findings addressed research question $1 \mathrm{~b}$.

Pioneering (essential theme one). Karen, Emily, Caroline, Jessica were pioneering because they recognized symptoms and markers that are clearly established in the research literature but not recognized by the professionals they sought help from. Jessica said having an early childhood degree was a "good and a bad thing." About the early signs of autism, she said "I could see it but not everyone [else could]." She said "..if [pediatricians] don't have hands on experience specifically to autism [in young children] it may not be visible." Jessica said that even her husband who is a physician at first thought "maybe you're too quick to call [it autism]." Emily expressed a similar opinion as Jessica. She felt that a lot of people thought that autism under three years old was "too young" and often people believe "[in our society parents are too] quick to think their child has autism." A teacher told her "I've never heard of two year olds getting diagnosed [with autism] before."

As described above participants were the first to recognize markers of autism for their very young children. Participants felt their efforts and persistence had a positive 
impact on the way their service providers and community viewed early autism after the fact. At the universal member check meeting participants reported that their early efforts "forging a trail" changed the way providers viewed early autism. Karen reported, "my doctor's practice is totally different now....She's referring way early now. She's like 'you know what I don't see it but... [you should get an evaluation] "Jessica had a similar experience, “They didn't listen--And so now everybody's like oh maybe we should have listened....but we can't rewind the time. But you know at least we can go forward.” The pioneering metaphor was further reinforced once the parents shared the knowledge with other families just starting their journey to autism diagnosis. Participants transmitted their knowledge about early markers and service navigation to other parents through social media and online autism organizations run by parents. These systems of communication and support were not found in the literature review. This likely reflects the relatively recent access to social media information networks and research to publication timelines. This finding has implications for support and resource systems for parents of very young children with autism.

Making the journey as a family (essential theme two). Family belonging and cohesion emerged as two subsidiary themes centering on immediate and extended family members. Participants reported family cohesion and belonging. This experience may have had an insulating effect, protecting family members from the effects of stigmatization and invalidation. The literature review yielded some insight on to the impact of a child's autism on family life. Cocoran, Berry, and Hill (date) reported on challenges associated with logistics, scheduling, and managing services. In their 14-study 
review of lived experiences they reported parental concern for other siblings. Consistent with my study's findings, Marinez (2009) found that relationships between spouses was enhanced and strengthened by their experiences during the early years with their children with autism. Their spouses were one of the greatest sources of support. My study yielded similar findings with husbands validating and providing emotional and practical support. Emily commented that "[some people say] having a child with autism can break up marriages, but it has brought [ours] closer together."

The findings from my study indicated extended family relationships were more complicated than immediate family ones. All parents faced questioning or invalidation from extended family, particularly with older generation members. Most of the questioning or invalidation centered on a lack of understanding about early symptoms of autism. Because the children either demonstrated poor social communication or had waxing and waning engagement, extended family members, often geographically separated, questioned whether the parents were being too lenient, over reacting, or falling prey to their view that "everyone thinks their child has autism." Participants displayed patience and supported families in understanding the child with autism's unique way of interacting in the world. Towards the end of the family journey (i.e., first three years) many of the extended family members understood and showed support of the participants' advocacy and decisions for their children.

Navigating the undeveloped new world of autism service systems for very young children (essential theme three). None of the participants in my study had a central service coordinator overseeing all of their child's services. They were the de 
facto service coordinators. The participants coordinated services across medical, educational, and county systems. They also interfaced with insurance companies, the Oregon Health Plan, respite workers, parent training programs, and other agencies. Parents also worked with pre-schools and communities to help insure their child's successful inclusion and participation. They acted as advocates about autism and what to expect from their child. They educated others on adaptations and engagement strategies to make interactions successful. When Caroline said there is no way to separate parenting and autism services I came to see what she meant. As pioneers there were few systems in place for very young children with autism so their daily life involved a vigilant management of existing services and focused attention to future service needs.

Educational programs. A recent study of parent perspectives on intervention services for infants/toddlers with ASD found that increased services and resources resulted in greater child outcomes (Bradshaw et al., 2015). Parents of infants/toddlers with autism viewed EI as effective when they were provided information about ASD, child development, and community services and resources (Cridland et al., 2014). This finding was consistent with the lived experiences of participants in my study. Parents reported that educational services, both EI and ECE, were underfunded and as a result, "meaningless," "abysmal," and "a waste of time." All parents received some sort of educational EI (i.e., speech delay or global developmental delay). Participants reported that the services were not helpful and there just weren't enough resources to make a difference. Emily received weekly home visits to increase her son's play skills and social interaction. The other three parents reported receiving 1 hour a month of EI home visiting 
services. Caroline raised the question of whether EI could be effective on this schedule.

She described the problems with a sparse intervention schedule with very young children:

CAROLINE: Once a freaking month or less than once a month...like 8 appointments does not equal 8 appointments because you have to orient yourself to the child and get the child comfortable with you to even do any intervention. And of course they're not -they're not in the same space as they were the last time you saw them. You know they've grown and developed or...um they don't remember from the last time....So it's completely an ineffective model of services.

Upon reviewing state outcome data for EI / ECSE for 2014 to 2016, Oregon State Legislature (OLS) recommended one hour per week in home or community visit for EI for every child receiving IDEA, Part C services (i.e., services birth to three) (Oregon Department of Education, [ODE], 2015). However, in Oregon infants and toddlers received less that $30 \%$ of the recommended service hours from 2014-2016 (OLIS, 2017). This trend continued when the children entered special education programs (ECSE). OLS recommended service hours for this age group were tiered by level of child need. There was an increasing disparity based on level of need between recommended service hours and services received. Table 8 compares state recommendations for ECSE services in Oregon and percentage of Oregon children whose services met service level standards. As the table indicates the greater the need, the lower the percentage of children receiving state level standards. The participants children in this study received well below the recommended service level for ECSE. 
Table 8

Recommended Service Levels for Oregon Children Receiving Early Childhood Special Education, Ages Three to Five (2016 data)

\begin{tabular}{|c|c|c|}
\hline $\begin{array}{l}\text { Child Level } \\
\text { of Need }\end{array}$ & $\begin{array}{c}\text { State Recommended } \\
\text { Services }\end{array}$ & $\begin{array}{l}\text { \% OR Children } \\
\text { Receiving Service } \\
\text { Level Standards }\end{array}$ \\
\hline $\begin{array}{l}\text { Low: } \\
\text { Delayed across } 1-2 \text { areas of } \\
\text { development (e.g. speech) }\end{array}$ & - 1 x week & $60 \%$ \\
\hline $\begin{array}{l}\text { Moderate: } \\
\text { Delayed in } 3-4 \text { areas of } \\
\text { development }\end{array}$ & $\begin{array}{l}\text { - } 3 \times \text { week preschool (12 } \\
\text { hours) } \\
\text { - } 1 \text { x week preschool } \\
\text { consultation } \\
\text { - } 1 \text { x month family } \\
\text { education }\end{array}$ & $<10 \%$ \\
\hline $\begin{array}{l}\text { High: } \\
\text { Delayed in almost every area } \\
\text { of development. }\end{array}$ & $\begin{array}{l}\text { - } 15 \text { hours week } \\
\text { preschool } \\
\text { - } 1 \text { x week preschool } \\
\text { consultation } \\
\text { - } 1 \text { x month family } \\
\text { consultation }\end{array}$ & $<5 \%$ \\
\hline
\end{tabular}

Oregon EI / ECSE outcome data. Results of this study regarding quality and intensity of educational services birth to 5 may have implications for interpreting outcome data for EI / ECSE programs in Oregon.. Oregon uses annual test scores from the Ages and Stages Questionnaire (ASQ; Bricker et al., 1999) and the Assessment, Evaluation, and Programming System for Infants and Children (AEPS; Bricker, 1994) to meet the federal child outcome reporting requirements for EI/ECSE. Participants in my study reported that there weren't enough EI/ECSE resources and as a result they felt the services did make much of a difference in their children's' development. The children in 
this study participated in an average of 20 treatment hours per week. However EI/ ECSE programs provided less than $25 \%$ of these treatment hours. Parents piecemealed the other $75 \%$ of services through medical insurance covered services, private preschools, county programs, Affordable Care Act, and other programs. It is unclear how much of a child's progress in educational programs as measured by the ASQ and AEPS was the result of the services they provide. The outcome data for these programs may not provide the federal government an accurate picture of the efficacy of EI/ECSE programs.

Services associated with positive parental experiences. All of the parents had positive experiences with a speech pathologist either within the educational program or, more often, through medical insurance. Each parent reported feeling that a speech therapist understood their situation and helped them obtain evaluations or other services. Caroline said that she "went against [her] pediatrician's back" and got a speech evaluation. She said she begged the speech pathologist to assist her family by noting that "we need help!" During that appointment, the therapist called and found out about a cancellation for an autism evaluation and helped get Nathan in to an appointment sooner. Karen reported that she still contacted Carter's EI speech therapist and sought advice from her when Carter's augmentative communication system was in jeopardy. All of the children in this study received speech services before autism-specific services. This finding may be linked to the timing of service acquisition and the trajectory of autism symptom emergence. All children began services (i.e., not autism-specific) around 24 months when they would have met criteria for language delay.

Emily and Karen's children participated in ABA services through medical 
insurance. Jessica wanted Helen to attend ABA and it was not covered under her husband's insurance plan. Caroline's ABA was ended abruptly and prior to that her feelings about ABA was neutral. Emily and Karen reported that they felt ABA was a great benefit to their children.

Overcoming obstacles and challenges (essential theme four). Parents in this study expressed exhaustion, frustration, stress, and grief. These findings were consistent with other studies of parents with children with autism. The literature review in Chapter 2 yielded research (Coogle, 2013; Corcoran et al., 2015; Osborne, McHugh, Saunders, \& Reed, 2008 ) finding parents often experienced services as a source of frustration and stress, My study was the one of first to explore this topic deeply. My study offers insight into the ways complex systems, service dimensions, and attitudes contribute to dissatisfaction, frustration, and stress. Some studies in the literature reported parents showed a strong desire for involvement in intervention and felt uncertainty in their role in developing EI plans for their child (Applequist \& Baily, 2000; Coogle, 2013). Grindle et al. (2009) found parents of children with autism desired social networks and relationships with other families with young children with autism. My study yielded insight into some of the ways complex ecological systems contributed to feelings of isolation, stigmatization, and stress. Participants repeatedly stressed how service system bureaucracy, lack of funding, and lack of validation made life exhausting. Parents worried about the impact of wait times on their children's developmental outcomes. They had to restructure their family lives in response to unexpected service changes. The participants in my study were adept at navigating services across many systems and 
described it as "a constant battle" and "a fight."

Obstacles and challenges resulting from their child having autism. While participants expressed worry, sadness, or grief over their children's autism they described these as fleeting moments and not prominent in their lives. Grief and stress associated with autism emerged as a trend in the research literature with older children (BakerEriczén, Brookman-Frazee, \& Stahmer, 2005; Kasari \& Sigman, 1997; Schieve, Blumberg, Rice, Visser, \& Boyle, 2007; Yamada et al., 2007). However, the literature review yielded little research on the experiences of parents with children with emergent autism. Nelson (2002) conducted a meta-synthesis of qualitative studies on mothering children with disabilities. The meta-synthesis revealed that while grief, stress, and fear were present, those feelings were often associated with the initial phase of adjusting to the child's diagnosis. In a mixed-method study, Green (2007) found mothers were satisfied with their relationship with their children with disabilities, but felt burdened by socio-cultural constraints. These older studies may reflect the sociocultural factors and view of autism at the time of the study. Through an ecological systems lens, my participants' fleeting and momentary grief may be associated with an increased acceptance and awareness of early autism in recent years. Overall parents attributed grief to the way the "world is built" and "lack of supports."

Ingenuity, resilience, and perseverance (essential theme five). This essential theme shed light on everyday lives of parents with young children with autism. Parents moved from accommodating their child's early needs (pre diagnosis) to advocacy (post diagnosis). Parents were ingenious in the strategies they used to support their children's 
participation in typical childhood activities. This may be related to the ways in which parents recognize and intuitively respond to their child's needs. The literature provided evidence that parents of children with autism are better at recognizing sensory processing difficulties in their children than parents of typically developing children (Dickie, Baranek, Schultz, Watson, \& McComish, 2009). This qualitative descriptive study also found these parents reported higher sensory aversions in their children and linked them to autism. Studies have also found parents of infants and toddlers with autism begin compensatory strategies years before an autism diagnosis. Wan and colleagues (2012) found parents of infants who went on to receive an ASD diagnosis became more directive with their young children. Parents of very young children with autism also provide more verbal scaffolding in increased prompting of social-communication (Sigman \& Siller, 2002). These findings were consistent with participant reports in my study. Karen realized that driving and keeping the car running soothed Carter when he was unable to regulate and sleep more than two hours a night. While many parents of infants drive their child to lull them to sleep, Karen describes a more intense experience than typical. Her experience with Carter's sensory processing problems was consistent with Dickie et al.'s (2009) finding that parents of children with autism described more extreme sensory responses and unusual behavioral responses than parents of children with typical development.

Participants' scaffolding strategies were practical and ingenious. Emily had to bolt and set up an alarm on her front door because when the family wasn't looking Joshua would "bolt across the street." Caroline recognized a feeding problem, sought the right 
doctor, and Nathan had surgery to correct a congenital oral anomaly (i.e., tongue-tie). Jessica was ingenious when she used a cat analogy to introduce Helen to her baby sister. She explained:

JESSICA: [We told Helen] 'Don't worry. This is just like another cat. She doesn't meow but it's ok...[see] she has black hair. See the cat has black hair' and [Helen's] like 'OK.'--it's almost like a cat meeting another cat. -- slowly slowly slowly getting into....another person's territory.

This finding suggests that parents are finding strategies to support their children's developmental goals even before starting EI/ECSE services. A tenant of family-centered practices is to involve parents in the intervention and embed goals into family routines. First generation EI models, given the emphasis on identifying treatment variables associated with success, tended to be manualized and less flexible than EI practices with other families with children with disabilities. Harnessing parent ingenuity in identifying early autism markers, scaffolding strategies, and intuitive responding may serve to improve child development and increase parent's sense of self efficacy (Bradshaw et al., 2015; Coogle et al., 2013).

\section{Reflecting on the first three years and looking forward. (essential theme six).}

Reflection and looking ahead emerged as the final essential theme. This phase marked the end of the journey from concerns about their children's development to arriving at services. An interesting trend across parents was their shared view that one of the most valuable functions of EI / ECSE services (birth to 5) was getting their foot in the door for special education services for kindergarten and beyond. They expressed that getting their children in the system early would make the transition to kindergarten smooth. Caroline said she wanted a "paper trail" with Nathan's IFSP to seamlessly continue working on his 
developmental goals. Jessica expressed getting her foot in the door and despite some of her dissatisfaction she wanted to establish and maintain a good relationship with the school system. Karen and Emily stuck with unsatisfactory EI / ECSE services for similar reasons. This was a new finding in the literature and was surprising to me as a researcher. While the participants understood the importance of EI/ECSE they also felt it was a procedural necessity. This finding may have implications for transition planning earlier in special education programs from EI to ECSE and again from ECSE to kindergarten. Participants were thinking ahead for what their children would need to successfully begin kindergarten. Educational administrators might enhance school success by strengthening a pathway from EI/ECSE to school programs. Elementary school models may have an opportunity to address inclusion and parent partnership earlier in the process with a nuanced understanding of the role of parents in successful transition of their children with autism.

Parents in this study were concerned about the next phase of educational services for their child. Just as we were wrapping up the universal member check meeting, Karen shared that thinks families are very concerned about their children with special needs starting kindergarten:

KAREN: I just saw something online...one of the biggest things [the advocacy group FACT Oregon] gets calls about is going into kindergarten...because the parents are like super stressed...you're supposed to have a transition meeting and this that and the other...we had one even though now my kids been pulled out of school....we had one and the transition person never showed up. I've never even met [Carter's] autism specialist.... 


\section{Section Two: Implications, Future Directions, and Limitations}

\section{The Study's Findings in the Context of Bronfenbrenner's Ecological Systems}

\section{Theory}

The Ecological Systems Theory (Bronfenbrenner, 1979) offered a lens and means for situating the research problem and explaining the complexity of interacting systems in the lives of parents with very young children with autism. My study was one of the first to examine early autism in the context of ecological systems. The descriptive phenomenological methodology (Husserl, 1970; Vagle, 2014) allowed me to explore parents' lived-experiences through many lenses. The photo-elicitation and unstructured interview methodology facilitated flexible data collection as I explored the research questions. The preponderance of narrative data related to service experiences. Consistent with Bronfenbrenner's (1979) ECST and Dunst's Family Systems Model (1979), my study found that service systems exerted influence on the participants' capacity to support their child's development. Bronfenbrenner's (1979) child development model associates child development with the nested ecosystems within which the child's life is embedded. Parents in my study anecdotally connected lack of services and fears over impact on their child's development, particularly during the lag time between diagnosis and services (Baio, 2012; Elsabbagh \& Johnson, 2007). Emily summed up her feelings on the issue, "the [professionals] push parents on getting early intervention for their kids but then make you jump through hoops to get it." This section describes the implications of the findings within the ECST framework and is subdivided into proximal and distal systems of influence on child development. 


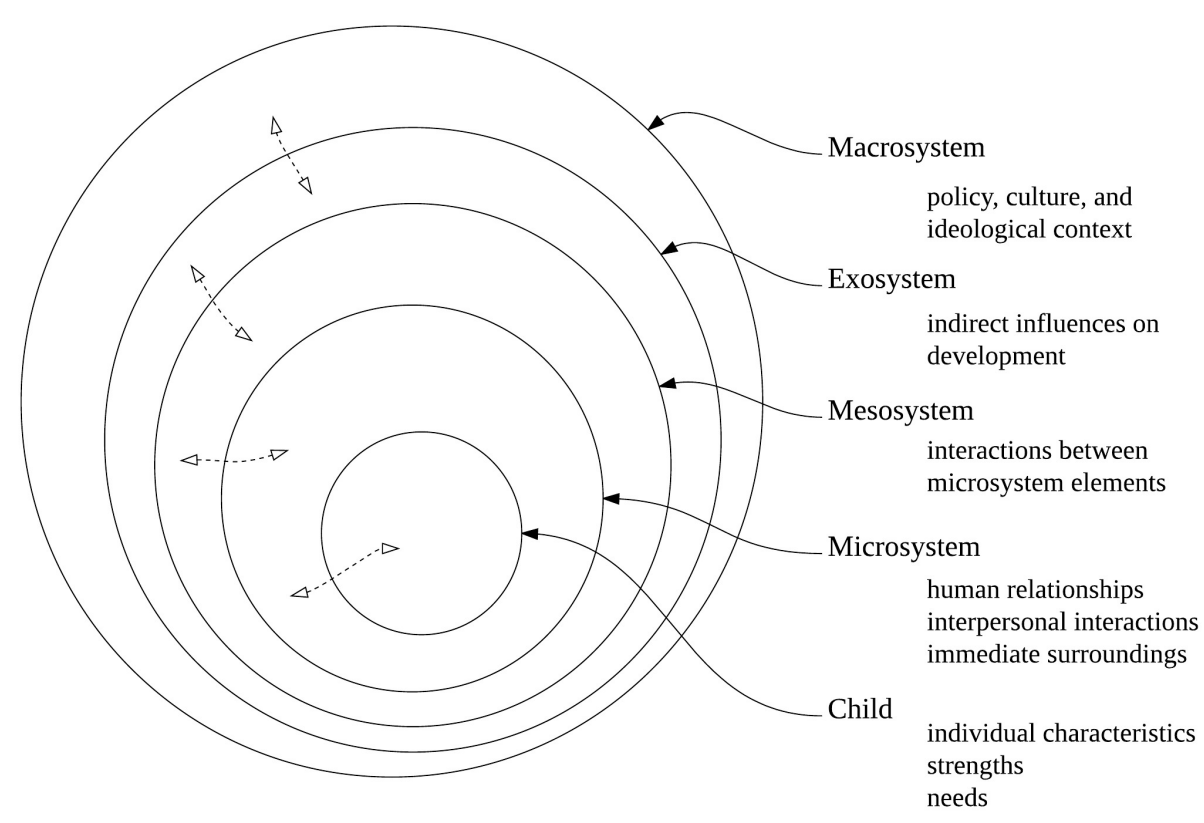

\section{Proximal ecosystems and study findings.}

Microsystem. The findings of my study described insufficient services and inadequate service systems for children with autism under age three. Consistent with the literature, parents recognized the early markers of autism. However, as parents sought help from pediatricians they did not receive support. While participants in my study received EI services for developmental delay or language delay, they did not received specialized autism services until age three. I found no research in the literature review on positive or negative effects of non-specialized EI for young children with autism.

However, parents in my study described their service experiences as largely inconsequential. In addition to EI service downfalls participants described lack of support from extended family during their child's first three years. Parents described isolation, exhaustion, and fatigue as they sought help for their child. On the other hand, parents described social media as the greatest type of support from early concerns through the 
diagnosis phase. The on-line communities (i.e., Facebook, local organization websites) provided information that may have mediated some of the effects microsystem level problem.

Mesosystem. The mesosystem represents the relationship between microsystems, typically between parent and other individuals and organizations that directly impact the child's development and access to support. Participants described the early stages of seeking help for their child as largely unsuccessful and "a constant battle." Each participant described persistence with pediatricians, continually pushing for help. They also conducted research to help advocate for their child. Once their child received an autism diagnosis the parents used the advocacy skills towards finding the right services, communicating with teachers, researching programs, and managing services. These experiences consumed a great deal of time and became the central experience in their family's life.

\section{Distal ecosystems and study findings.}

Macrosystem. The cultural and political environments exert influence on child development (Bronfenbrenner, 1979). Participants described misconceptions by others about early manifestations autism. They described a proximal and distal culture of disbelief and judgment. The literature and policy review for this study corroborated the results from participants' conversations. Stahmer and Mandell (2007) found little evidence for AUT-EI across the U.S. states. In Oregon infants and toddlers with disabilities receive only $25 \%$ of recommended service levels (OLIS, 2014). This trend continued as the children transitioned into early childhood special education programs 
(i.e., ages 3 to 5).

Chronosystem. Bronfenbrenner described the choronsystem as a distal influence on child development. The chronosystem encompasses the time in which the child is being raised and also the age of the child during which the lived-experiences occurred (e.g., child age, significant events in child's life, socio-historical context). The current emergent state of AUT-EI clearly had implications for the parents in my study. In fact, ignorance about early manifestations of autism led to invalidation of participants' concerns and denial of early intervention services for their children. Historically children with autism and their parents have been viewed from a pathology model. In the 1940s and 1950s researchers often described autism as a psychological pathology resulting from unresponsive, cold parenting practices. Some scholars argue that the vestiges of these negative attitudes continue to exert influence on parents and children's lived experiences.

Subsection summary. The results from my study demonstrated the utility of phenomenological methodology in understanding the complex and inter-related systems exerting influence on parenting a young child with autism. Just as Bronfenbrenner (1979) illustrated and described the complex environmental influence on child development, my findings reinforce a contextualized and sometimes socially constructed view of disability that influences developmental outcomes in children with autism. These influences mediate parent capacity to support their child's development. Dunst and Trivette's (2009) Family Systems Framework illustrates the role of capacity building in helping parents support their infant and toddler's development. A qualitative understanding of influences on parenting and intervening in AUT-EI may inform practices, research and advocacy 
efforts.

\section{Future Directions}

Practice implications. The lived experiences and essential themes from this study have implications for future research and practice. Given the rich and detailed stories captured by this study, there is opportunity to disseminate this information about parenting a child with autism. Other parents might benefit from reading about participants' first observations and feelings about their child's development. The stories in their candor and detail may offer a road map and reassurance to other parents; simply hearing the participant's stories of family life and service navigation may be a validating experience for others. Through publication in academic and layperson journals and magazines, the parent's voices may reach others. Given the emphasis on early intervention and the emergent nature of autism in infants and toddlers, the findings of this study offer an opportunity for providers to see the service process through the eyes of the participants. This vantage might inform practices that address lack of service availability, need for service coordination, and promote advocacy efforts to increase funding and services. There are many ways the findings of this study can be utilized to improve the lives of parents of very young children with autism including sharing participants stories in articles, training direct service providers, and creating materials to support advocacy efforts. Finally, my hope is that the process itself was beneficial to the participants. While their voice is important in the future of research and practice, it might have served as validation of their experiences. 
Testing hypotheses generated from the results of this study. Qualitative research in special education plays an important role in generating hypotheses that can later be tested and confirmed through quantitative means (Given, 2008). Given the robust service navigation themes in this study, the corroborating research from the literature, and indications that Oregon does not deliver the quantity of services recommended to improve child developmental outcomes (ODE, 2015), a quantitative design survey examining variables associated with the existing ODE findings may be an important next step in advocating for more services at the state and federal level. Based on the results of this study, it would also be worthwhile to include queries on the intersecting systems of care for very young children with autism.

\section{Replication of study with other populations. Recently some AUT-EI} researchers have turned their focus to the intersection between autism and underrepresented populations. Mandell, et al., 2009 reported racial disparities in autism recognition and diagnosis in young children. African American children with autism receive a diagnosis 1.6 years later than Caucasian children (Mandell et al., 2002). They are also 2.6 times more likely to be misdiagnosed and 5.1 times more likely to be incorrectly diagnosed with an adjustment disorder and 2.4 times more likely to be misdiagnosed with a conduct disorder (Mandell et al., 2007). My study explored the phenomenon of parenting a very young child with autism and navigating early service systems with a sample of educated, middle income parents. The parents in my study acknowledged the resources available to them because of these demographic 
characteristics. The essential themes may be very different for parents in underrepresented populations. For example, recognition of markers may come later. Advocacy efforts and acquisition of service are likely mediated by available time and resources that are commonly associated with economic factors. Generalizability is a stated limitation of my study. This issue may be especially important when extrapolating lived experiences across cultures, economic status, and other populations.

Longitudinal replication with same participants. Just as the essential themes may change across populations, they are likely subject to the influences of time. Within my study's parameters a temporal trend emerged across participants. They indeed were on a journey from recognition of symptoms to acquisition of autism services. However, as their children age, the families will likely face new challenges, triumphs, and lived experiences. Karen and Caroline both remarked on how others treat their children now that they are no longer "cute" and little toddlers. Extending this study longitudinally may add insight into the family and service needs beyond early childhood particularly since the preponderance of autism behavioral research is with preschool aged children and older children.

Family priorities and goals for children with autism evolve as children age. Successful kindergarten experiences require skills not developmentally appropriate for very young children (e.g., participating in group activities, following school routines, behavioral regulation). Middle schoolers with autism often face challenges with school cultures that are not inclusive and may result in social exclusion. Autism research drastically reduces once children are beyond school age (Wong et al., 2015). My study 
looked deeply at one phase of a parent's of a child with autism's life. It may be worthwhile to capture lived experiences during other life stages so a full picture of the phenomenon of autism and parenting may be documented and better understood.

Photo-elicitation utility in early intervention interviewing. Given the proliferation of cell phones and use of digital photography, photo elicitation offers an opportunity to incorporate family photographs into EI interviewing and data collection. Photo-elicitation offers EI specialists the opportunity to gathered nuanced information about family life, values, and priorities. In much the same way that photo-elicitation interviewing anchors participant memories and creates a connection with the researcher (Collier, 1957; Harper, 2002), early intervention professionals might benefit by incorporating family photographs into gathering information about family lifestyle and culture. Early intervention providers currently use tools to gather ecological data on family life to increase acceptability to parents and improve implementation (e.g., ecomapping (Noon \& McCormick, 1993); routines based interviewing (McWilliam, Casey, \& Sims, 2009).). Photo-elicitation interviewing would add a nuanced strategy for understanding parent perspectives. Collaboration is the cornerstone of early intervention and collection of ecological data is an established component of effective EI (Keilty, 2010) and linked to plan efficacy (Ketelaar, Vermeer, Helders, \& Hart, 1998; Shonkoff \& Hauser-Cram, 1987). Photographs may aid parents in sharing information that would otherwise be inaccessible. Further photo-elicitation methods neutralize power relationships between researchers and participants. This effect may generalize to EI professionals and result in stronger collaborative relationships with parents. 


\section{Limitations}

While the results of this study offered nuanced and descriptive insight into the lived experiences of parenting a very young child with autism and navigating the service systems, this study has limitations particularly with generalizability of the findings. The phenomenological methodology and the small sample size preclude conclusions about early autism services or parenting experiences beyond the sample. Further, the snowball recruitment strategy was effective at identifying participants with experience with the phenomena, however it also generated a group of participants who were geographically near to each other and who also shared middle-income backgrounds. The four participants were educated, with three having graduate level training. It is reasonable to assume that the lived experiences parenting a very young child with autism might be considerably different in other demographic, economic, or cultural backgrounds.

As a researcher I encountered an unexpected ethical conflict with the photoelicitation methodology. Consistent with research in the field the use of photo-elicitation in the interview process I felt the depth of information and positive effects on collaboration were valuable. However, as the study progressed I wished to use the photographs in the final presentation of results. I weighed the risks / benefits and ultimately found a way to digitally alter the photographs and amend the IRB. However, this experience reminded me of the vulnerability of this population of participants and their children. Further, I realized that there are ethical concerns with modifying consent for use of photographs at the conclusion of the study. I believe I effectively addressed this risk through a rigorous consent process and also through the trusting relationship I 
established with the participants.

I also learned about myself as a researcher. As a passionate advocate for families I sometimes risk bias interfering with my work. The descriptive phenomenological methodology and bracketing in particular allowed me to address these biases through reflection and repeated contact with the data and participants. For example, I entered the research process with criticisms of both the lack of funding for early intervention and also the what I considered the rigidity of Applied Behavior Analysis treatments. My first criticism / bias was confirmed but also more nuanced than I expected. All parents confirmed EI services they received were insufficient. However, parents expressed a desire to have a positive relationship with early education providers and felt the professionals were stretched too thin. I was also surprised that the three parents receiving ABA interventions were happy with the positive effects on their children's development, particularly language and social outcomes.

\section{Chapter 5 Summary}

The findings of this study highlighted the inextricability of autism, service delivery, and parenting during the phenomenon of parenting a very young child with autism receiving EI services. Much can be garnered from the lived experiences of participants and their tireless efforts to find the right services for their child. Many of these issues described by the participants were consistent with the research literature but there were also new findings from this study. The phenomenological and photo elicitation methodologies proved invaluable in exploring the authentic lived experiences of parents and were consistent with the photo elicitation literature. Recently, Karen sent me an 
email. She wrote "Thanks for doing this project... I really appreciate the voice you're giving us.” 


\section{REFERENCES}

Adolphs, R., Sears, L., \& Piven, J. (2001). Abnormal processing of social information from faces in autism. Journal of Cognitive Neuroscience, 13, 232-240. doi: $10.1162 / 089892901564289$

Adrien, J. L., Lenoir, P., Martineau, J., Perrot, A., Hameury, L., Larmande, C., \& Sauvage, D. (1993). Blind ratings of early symptoms of autism based upon family home movies. Journal of the American Academy of Child \& Adolescent Psychiatry, 32, 617-626. doi:10.1097/00004583-199305000-00019

Aldred C, Green J, \& Adams C. (2004). A new social communication intervention for children with autism: pilot randomised controlled treatment study suggesting effectiveness. Journal of Child Psychology \& Psychiatry, 45, 1420-1430. doi: 10.1111/j.1469-7610.2004.00338.x

American Psychiatric Association. (2013). Diagnostic and statistical manual of mental disorders: DSM 5. Washington, DC: American Psychiatric Association.

Apicella, F., Chericoni, N., Costanzo, V., Baldini, S., Billeci, L., Cohen, D., \& Muratori, F. (2013). Reciprocity in interaction: A window on the first year of life in autism. Autism Research and treatment, (2013), 1-12. doi: 10.1155/2013/705895

Applied Behavior Center for Autism [ABCA] (2016). Surgeon general endorses behavioral intervention for autism. Retrieved from: http://appliedbehaviorcenter.com/aba-endorsements/

Autistic Self Advocacy Network (ASAN) (2009). The Autistic Self Advocacy NetworkDedicated to advancing understanding, acceptance, and support for the autistic community. Retrieved from http://www.autisticadvocacy.org

Applequist, K. L., \& Bailey, D. B. (2000). Navajo caregivers' perceptions of early intervention services. Journal of Early Intervention, 23, 47-61. doi: $10.1177 / 10538151000230010901$

Autism Speaks (2016). State initiatives. Retrieved from https://www.autismspeaks.org/state-initiatives

Babbie, E. (1995). The practice of social research (7th ed.). Belmont, CA: Wadsworth. Baghdadli, A., Picto, M.C., Pascal, C., Pry, R., \& Aussilloux, C. (2003). Relationship between age of recognition of first disturbances and severity in young children with autism. European Child and Adolescent Psychiatry, 12, 122-127. doi: 10.1007/s00787-003-0314-6

Bailey, D. B. (1991). Family orientation of community and agency services [Measurement instrument]. Chapel Hill, NC: University of North Carolina.

Bailey, D. B., Hebbeler, K., Scarborough, A., Spiker, D., \& Mallik, S. (2004). First experiences with early intervention: a national perspective. Pediatrics, 113, 887896. doi: $10.1542 /$ peds. 113.4 .887

Bailey, D., Scarborough, A., \& Hebbeler, K. (2003). Families' first experiences with early intervention. Menlo Park, CA: SRI International.

Baio, J. (2012). Prevalence of autism spectrum disorders: Autism and developmental disabilities monitoring network, 14 sites, United States, 2008. Morbidity and Mortality Weekly Report [CDC]. Surveillance Summaries, 61(3), n.p. 
Baker-Ericzén, M. J., Brookman-Frazee, L., \& Stahmer, A. (2005). Stress levels and adaptability in parents of toddlers with and without autism spectrum disorders. Research and Practice for Persons with Severe Disabilities, 30, 194204. doi: $10.2511 / \mathrm{rpsd} .30 .4 .194$

Baranek, G. T. (1999). Autism during infancy: A retrospective video analysis of sensorymotor and social behaviors at 9-12 months of age. Journal of Autism and Developmental Disorders, 29, 213-224. doi: 10.1023/A:1023080005650

Barbaro, J., \& Dissanayake, C. (2013). Early markers of autism spectrum disorders in infants and toddlers prospectively identified in the Social Attention and Communication Study. Autism, 17, 64-86. doi: 10.1177/1362361312442597

Bayat, M. (2007). Evidence of resilience in families of children with autism. Journal of Intellectual Disability Research, 51, 702-714. doi: 10.1111/j.13652788.2007.00960.x

Beals, K. (2004). Early intervention in deafness and autism: One family's experiences, reflections, and recommendations. Infants \& Young Children, 17, 284-290.

Benner, P. (1985). A phenomenological perspective on explanation, prediction and understanding in nursing science. Advances in Nursing Science, 8, 1-14.

Ben-Sasson, A., Cermak, S. A., Orsmond, G. I., \& Tager-Flusberg, H. (2007). Extreme sensory modulation behaviors in toddlers with autism spectrum disorders. The American Journal of Occupational Therapy, 61, 584. Retrieved from: http://www.henryot.com/pdf/Behaviors_in_Toddlers_with_Autism.pdf

Bergström, E. B., Wallin, L., Thomson, G., \& Flacking, R. (2012). Postpartum depression in mothers of infants cared for in a neonatal intensive care unitIncidence and associated factors. Journal of Neonatal Nursing, 18, 143-151. doi: 10.1016/j.jnn.2011.11.001

Bernheimer, L. P., \& Keogh, B. K. (1995). Weaving interventions into the fabric of everyday life: An approach to family assessment. Topics in Early Childhood Special Education, 15, 415-433. doi: 10.1177/027112149501500402

Bernheimer, L. P., \& Weisner, T. S. (2007). "Let me just tell you what I do all day...": The family story at the center of intervention research and practice. Infants \& Young Children, 20, 192-201. doi: 10.1097/01.IYC.0000277751.62819.9b

Beurkens, N. M., Hobson, J. A., \& Hobson, R. P. (2013). Autism severity and qualities of parent-child relations. Journal of Autism and Developmental Disorders, 43, 168178. doi: 10.1007/s10803-012-1562-4

Bolte, S. (2014). The power of words: Is qualitative research as important as quantitative research in the study of autism? Autism, 8, 67-68. doi: $10.1177 / 1362361313517367$

Bornstein, M. H., \& Tamis-Lemonda, C. S. (1997). Maternal responsiveness and infant mental abilities: Specific predictive relations. Infant Behavior and Development, 20, 283-296. doi: 10.1016/S0163-6383(97)90001-1

Boyd, B. A., Odom, S. L., Humphreys, B. P., \& Sam, A. M. (2010). Infants and toddlers with autism spectrum disorder: Early identification and early intervention. Journal of Early Intervention, 32, 75-98. doi: $\underline{10.1177 / 1053815110362690}$ 
Bradford, K. (2010). Brief education about autism spectrum disorders for family therapists. Journal of Family Psychotherapy, 21, 161-179. Retrieved from: http://www-tandfonlinecom.proxy.lib.pdx.edu/doi/full/10.1080/08975353.2010.505529

Bradshaw, J., Steiner, A. M., Gengoux, G., \& Koegel, L. K. (2015). Feasibility and effectiveness of very early intervention for infants at-risk for autism spectrum disorder: A systematic review. Journal of Autism and Developmental Disorders, 45, 778-794. doi: 10.1007/s10803-014-2235-2

Brantlinger, E., Jimenez, R., Klingner, J., Pugach, M., \& Richardson, V. (2005). Qualitative studies in special education. Exceptional Children, 71, 195-207. doi: 10.1177/001440290507100205

Brazelton, T. B., Tronick, E., Adamson, L., Als, H., \& Wise, S. (1975). Early motherinfant reciprocity. Parent-Infant Interaction, (33), 137-154. doi: 10.1002/9780470720158.ch9

Bricker, D. (1994). Assessment, Evaluation, and Programming System (AEPS) for infants and children. Volume 1: Measurement for birth to three Years [Measurement Instrument\}. Baltimore, MD: Brookes Publishing.

Bricker, D., Squires, J., Mounts, L., Potter, L., Nickel, R., Twombly, E., \& Farrell, J. (1999). Ages and stages questionnaire (ASQ) [Measurement Instrument]. Baltimore, MD: Brookes Publishing.

Broderick, A. A. (2011). Autism as rhetoric: Exploring watershed rhetorical moments in applied behavior analysis discourse. Disability Studies Quarterly,31(3) n.p. doi: 10.18061/dsq.v31i3.1674

Brogan, C. A., \& Knussen, C. (2003). The disclosure of a diagnosis of an autistic spectrum disorder determinants of satisfaction in a sample of Scottish parents. Autism, 7, 31-46. doi:10.1177/1362361303007001004

Bronfenbrenner, U. (1979). The ecology of human development: Experiments by design and nature. Boston, MA: Harvard University Press.

Bronfenbrenner, U. (1992). Ecological systems theory. In R.Vasta (Ed.), Six theories of child development: Revised formulations and current issues (pp. 187-248). Philadelphia, PA: Jessica Kingsley.

Bronfenbrenner, U. (1998). The ecology of developmental process. In P. A. Damon \& W. Lerner, R.M. (Eds), Handbook of child psychology: Theoretical models of human development (5th ed.) (pp. 993-1028). Hoboken, NJ: John Wiley \& Sons.

Brooks-Gunn, J.M. (1995). Children in families in communities: Risk and intervention in the Bronfenbrenner tradition. In P. Moen, G. Elder, \& K. Lüscher (Eds.), Examining lives in context: Perspectives on the ecology of human development. (pp. 467-519). Washington, DC: American Psychological Association.

Bruder, M. B. (2000). Family-centered early intervention: Clarifying our values for the new millennium. Topics in Early Childhood Special Education (20), 105-115. doi: $10.1177 / 027112140002000206$

Bryson, S. E., Zwaigenbaum, L., Brian, J., Roberts, W., Szatmari, P., Rombough, V., \& McDermott, C. (2007). A prospective case series of high-risk infants who 
developed autism. Journal of Autism and Developmental Disorders, 37, 12-24. doi: 10.1007/s10803-006-0328-2

Byrne, E., Daykin, N., \& Coad, J. (2016). Participatory photography in qualitative research: A methodological review. Visual Methodologies, 4(2), 1-12. doi: 10.7331/vm.v4i2.66

Burch, R. (1944). Phenomenology, lived experience: Taking a measure of the topic. Phenomenology and Pedagogy, 8, 130-160. Retrieved from: https://journals-library-ualbertaca.proxy.lib.pdx.edu/pandp/index.php/pandp/article/viewFile/15137/11958

Camarata, S. (2014). Early identification and early intervention in autism spectrum disorders: Accurate and effective? International Journal of Speech-Language Pathology, 16, 1-10. doi: 10.3109/17549507.2013.858773

Carbone, P. S., Behl, D. D., Azor, V., \& Murphy, N. A. (2010). The medical home for children with autism spectrum disorders: Parent and pediatrician perspectives. Journal of Autism and Developmental Disorders, 40, 317-324. doi: 1007/s10803-009-0874-5

Carter, A. S., Messinger, D. S., Stone, W. L., Celimli, S., Nahmias, A. S., \& Yoder, P. (2011). A randomized controlled trial of Hanen's 'More Than Words' in toddlers with early autism symptoms. Journal of Child Psychology and Psychiatry, 52, 741-752. doi: 10.1111/j.1469-7610.2011.02395.x

Center for Disease and Control (CDC). (2014) Prevalence of autism spectrum disorders among children aged 8 years: Autism and developmental disabilities monitoring network, 11 sites, United States, 2010. MMWR Surveillance Summaries 63(2): 122.

Chawarska, K., Paul, R., Klin, A., Hannigen, S., Dichtel, L. E., \& Volkmar, F. (2007). Parental recognition of developmental problems in toddlers with autism spectrum disorders. Journal of Autism and Developmental Disorders, 37, 62-72. doi: 10.1007/s 10803-006-0330-8

Chevallier, C., Kohls, G., Troiani, V., Brodkin, E. S., \& Schultz, R. T. (2012). The social motivation theory of autism. Trends in Cognitive Sciences, 16, 231-239. doi: 10.1016/j.tics.2012.02.007

Clark-Ibáñez, M. (2004). Framing the social world with photo-elicitation interviews. American Behavioral Scientist, 47, 1507-1527. doi: $10.1177 / 0002764204266236$

Colaizzi, P. F. (1978). Psychological research as the phenomenologist views it. In R. S. Valle \& M. King (Eds.), Existential-phenomenological alternatives for psychology (pp. 48-71). New York, NY: Oxford University Press.

Collier, J. Jr. (1957). Photography in anthropology: A report on two experiments. American Anthropologist, New Series, 59, 843-859. doi: 10.1525/aa.1957.59.5.02a00100

Coogle, C., Guerette, A. R., \& Hanline, M. F. (2013). Early intervention experiences of families of children with an autism spectrum disorder: A qualitative pilot study. Early Childhood Research \& Practice, 15, 1-12. Retrieved from: https://eric.ed.gov/?id=EJ1016155 
Coogle, C. G., \& Hanline, M. F. (2014). An exploratory study of family-centred helpgiving practices in early intervention: Families of young children with autism spectrum disorder. Child \& Family Social Work, 21, 249-260. doi: $10.1111 / \mathrm{cfs} .12148$

Copple, C., \& Bredekamp, S. (2009). Developmentally appropriate practice in early childhood programs serving children from birth through age 8. Washington, DC: National Association for the Education of Young Children.

Corbin, J., \& Morse, J. M. (2003). The unstructured interactive interview: Issues of reciprocity and risks when dealing with sensitive topics. Qualitative Inquiry, 9, 335-354. doi: 10.1177/1077800403009003001

Corcoran, J., Berry, A., \& Hill, S. (2015). The lived experience of US parents of children with autism spectrum disorders: A systematic review and meta-synthesis. Journal of Intellectual Disabilities, 19, 356-366. doi: 10.1177/1744629515577876

Courchesne, E., Campbell, K., \& Solso, S. (2011). Brain growth across the life span in autism: Age-specific changes in anatomical pathology. Brain Research, 1380, 138-145. doi: 10.1016/j.brainres.2010.09.101

Crabtree, B. F., \& Miller, W. L. (Eds.). (1992). Doing qualitative research: Research methods for primary care. Newbury Park, CA: Sage.

Cresswell, J.W. (2005). Educational research: Planning, conducting, and evaluating. Upper Saddle River, NJ: Pearson.

Cridland, E.K., Jones, S.C., Caputi, P., \& Magee, C.A. (2014). Qualitative research with families living with autism spectrum disorder: Recommendations for conducting semi-structured interviews. Journal of Intellectual and Developmental Disability, 40, 78-91. doi: 10.3109/13668250.2014.964191

Cunningham, C. C. (1987). Early intervention in Down's syndrome. In G. Hoskins \& G. Murphy (Eds.), Prevention of mental handicap: A world view (pp. 169-182). London: Royal Society of Medicine Services.

Dahlgren, S. O., \& Gillberg, C. (1989). Symptoms in the first two years of life. European Archives of Psychiatry and Neurological Science, 238, 169-174. doi: 10.1007/BF00451006

Dalton, K. M., Nacewicz, B. M., Johnstone, T., Schaefer, H. S., Gernsbacher, M. A., Goldsmith, H. H., ... \& Davidson, R. J. (2005). Gaze fixation and the neural circuitry of face processing in autism. Nature Neuroscience, $8,519-526$. doi: $10.1038 / \mathrm{nn} 1421$

Davis, N. O., \& Carter, A. S. (2008). Parenting stress in mothers and fathers of toddlers with autism spectrum disorders: Associations with child characteristics. Journal of Autism and Developmental Disorders, 38, 1278-1291. doi: 10.1007/s10803007-0512-zdoi: 10.1007/s10803-007-0512-z

Dawson, G. (2008). Early behavioral intervention, brain plasticity, and the prevention of autism spectrum disorder. Development and Psychopathology, 20, 775-803. doi: $10.1017 / \mathrm{S} 0954579408000370$

Dawson, G., Webb, S. J., Wijsman, E., Schellenberg, G., Estes, A., Munson, J., \& Faja, S. (2005). Neurocognitive and electrophysiological evidence of altered face processing in parents of children with autism: Implications for a model of 
abnormal development of social brain circuitry in autism. Development and Psychopathology, 17, 679-697. doi: 10.1017/S0954579405050327

De Chesnay, M. (2014). Nursing research using participatory action research: Qualitative designs and methods in nursing. New York, NY: Springer.

De Giacomo, A., \& Fombonne, E. (1998). Parental recognition of developmental abnormalities in autism. European Child \& Adolescent Psychiatry, 7, 131-136. doi: $10.1007 / \mathrm{s} 007870050058$

Dellenmark-Blom, M., \& Wigert, H. (2014). Parents' experiences with neonatal home care following initial care in the neonatal intensive care unit: A phenomenological hermeneutical interview study. Journal of Advanced Nursing, 70, 575-586. doi: $10.1111 /$ jan. 12218

Dennis, S. F., Gaulocher, S., Carpiano, R. M., \& Brown, D. (2009). Participatory photo mapping (PPM): Exploring an integrated method for health and place research with young people. Health \& Place, 15, 466-473. doi: 10.1016/j.healthplace.2008.08.004

Denzin, N. K. (1973). The research act: A theoretical introduction to sociological methods. Piscataway, NJ: Transaction.

DePape, A.M. \& Lindsay, S. (2015). Parents' experiences of caring for a child with autism spectrum disorder. Qualitative Health Research, 25, 569-583. doi: $10.1177 / 1049732314552455$

Dew, A., Balandin, S., \& Llewellyn, G. (2008). The psychosocial impact on siblings of people with lifelong physical disability: A review of the literature. Journal of Developmental and Physical Disabilities, 20, 485-507. doi: 10.1007/s10882-0089109-5

Dickie, V. A., Baranek, G. T., Schultz, B., Watson, L. R., \& McComish, C. S. (2009). Parent reports of sensory experiences of preschool children with and without autism: A qualitative study. American Journal of Occupational Therapy, 63, 172181. doi: 10.5014/ajot.63.2.172

Dillenburger, K., Keenan, M., Doherty, A., Byrne, T., \& Gallagher, S. (2010). FOCUS ON PRACTICE: Living with children diagnosed with autistic spectrum disorder: Parental and professional views. British Journal of Special Education, 37, 13-23. doi: 10.1111/j.1467-8578.2010.00455.x

Division for Early Childhood [DEC]. (2014). DEC recommended practices in early intervention/early childhood special education. Retrieved from http://www.decsped.org/recommendedpractices

Dockett, S., Einarsdottir, J., \& Perry, B. (2009). Researching with children: Ethical tensions. Journal of Early Childhood Research, 7, 283-298. doi: 10.1177/1476718X09336971

Drew, A., Baird, G., Baron-Cohen, S., Cox, A., Slonims, V., Wheelwright, S., ... \& Charman, T. (2002). A pilot randomised control trial of a parent training intervention for pre-school children with autism. European Child \& Adolescent Psychiatry, 11, 266-272. doi: 10.1007/s00787-002-0299-6

Dunn, W. (1997). The impact of sensory processing abilities on the daily lives of young children and their families: A conceptual model. Infants \& Young Children, 9(4), 
23-35 Retrieved from: https://search.library.pdx.edu/primo-

explore/fulldisplay?docid=TN_ericEJ546008\&context=PC\&vid=PSU\&search_sc ope $=$ all\&tab $=$ default tab\&lang $=$ en_US

Dunst, C. J. (2000). Revisiting" Rethinking Early Intervention". Topics in Early Childhood Special Education, 20, 95-104. doi: 10.1177/027112140002000205

Dunst, C. J., \& Trivette, C. M. (1989). An enablement and empowerment perspective of case management. Topics in Early Childhood Special Education, 8, 87-102. doi: $10.1177 / 027112148900800407$

Dunst, C. J., \& Trivette, C. M. (2009). Capacity-building family-systems intervention practices. Journal of Family Social Work, 12, 119-143. doi: 10.1080/10522150802713322

Dunst, C.J., Trivette, C.M., \& Deal, A.G. (1994). Resource-based family-centered intervention practices. In C.J. Dunst, C.M. Trivette, \& A.G. Deal (Eds.).

Supporting and strengthening families: Methods, strategies \& practices (pp. 140151). Cambridge, MA: Brookline Books.

Dunst, C. J., Trivette, C. M., \& Hamby, D. W. (2007). Meta-analysis of family-centered helpgiving practices research. Mental Retardation and Developmental Disabilities Research Reviews, 13, 370-378. doi: 10.1002/mrdd.20176

Eatough, V., \& Smith, J.A. (2008). Phenomenological psychology. In A.P. Giorgi \& B. Giorgi, (Eds). The SAGE handbook of qualitative research in psychology, (pp. 165-179). Thousand Oaks, CA: Sage.

Egeland, B., \& Farber, E. A. (1984). Infant-mother attachment: Factors related to its development and changes over time. Child development, 753-771. doi: $10.2307 / 1130127$

Eldevik, S., Hastings, R. P., Hughes, J. C., Jahr, E., Eikeseth, S., \& Cross, S. (2009). Meta-analysis of early intensive behavioral intervention for children with autism. Journal of Clinical Child \& Adolescent Psychology, 38, 439-450. doi: 10.1080/15374410902851739

Elsabbagh, M., \& Johnson, M. H. (2007). Infancy and autism: Progress, prospects, and challenges. Progress in Brain Research, 164, 355-383. doi: 10.1016/S00796123(07)64020-5

Elsabbagh, M., \& Johnson, M. H. (2010). Getting answers from babies about autism. Trends in Cognitive Sciences, 14, 81-87. doi: 10.1016/j.tics.2009.12.005

Epley, P. H., Summers, J. A., \& Turnbull, A. P. (2011). Family outcomes of early intervention: Families' perceptions of need, services, and outcomes. Journal of Early Intervention, 33, 201-219. doi: 10.1177/1053815111425929

Erikson, E. H. (1993). Childhood and society. New York, NY: Norton \& Company.

Estes, A., Munson, J., Dawson, G., Koehler, E., Zhou, X. H., \& Abbott, R. (2009). Parenting stress and psychological functioning among mothers of preschool children with autism and developmental delay. Autism, 13, 375-387. doi: $10.1177 / 1362361309105658$

Farrell, A. F. (2009). Validating family-centeredness in early intervention evaluation reports. Infants \& Young Children, 22, 238-252. doi:10.1097/IYC.0b013e3181b8b1db 
Fegran, L., Fagermoen, M. S., \& Helseth, S. (2008). Development of parent-nurse relationships in neonatal intensive care units-From closeness to detachment. Journal of Advanced Nursing, 64, 363-371. doi: 10.1111/j.13652648.2008.04777.x

Fenton, A., \& Krahn, T. (2009). Autism, neurodiversity and equality beyond the'normal'. Journal of Ethics in Mental Health, 2, 2-11. Retrieved from: http://philpapers.org.proxy.lib.pdx.edu/rec/FENANA.

Flanagan, H. E., Smith, I. M., Vaillancourt, T., Duku, E., Szatmari, P., Bryson, S., ... \& Waddell, C. (2015). Stability and change in the cognitive and adaptive behaviour scores of preschoolers with autism spectrum disorder. Journal of Autism and Developmental Disorders, 45, 2691-2703. doi: 10.1007/s10803-015-2433-6 doi: 10.1007/s10803-015-2433-6doi: 10.1007/s10803-015-2433-6doi: $10.1007 / \mathrm{s} 10803-015-2433-6$

Foucault, M. (1988). Practicing criticism. In L. Kritzman (ed.), Michel Foucault: Politics, philosophy, culture, (pp. 154-162). New York, NY: Routledge.

Fouche, F. (1993). Phenomenological theory of human science. In J. Snyman (Ed.), Conceptions of social inquiry (pp. 87-112). Pretoria, South Africa: Human Science Research Council.

Gearing, R. E. (2004). Bracketing in research: A typology. Qualitative Health Research, 14, 1429-1452. doi: $10.1177 / 1049732304270394$

Given, L. M. (2008). The SAGE encyclopedia of qualitative research methods. Thousand Oaks, CA: SAGE Publications Ltd

Glynne-Owen, R. (2010). Early intervention and autism: The impact of positivism and the call for change. The International Journal of Children's Rights, 18, 405-416. doi: $10.1163 / 157181810 X 497431$

Goin, R. P., \& Myers, B. J. (2004). Characteristics of infantile autism moving toward earlier detection. Focus on Autism and Other Developmental Disabilities, 19, 5 12. doi: 10.1177/10883576040190010201

Goin-Kochel, R. P., Mackintosh, V. H., \& Myers, B. J. (2006). How many doctors does it take to make an autism spectrum diagnosis?. Autism, 10, 439-451. doi: $10.1177 / 1362361306066601$

Gorgi, A. (2009). The descriptive phenomenological method in psychology: A modified Husserlian approach. Pittsburg, PA: Duquesne University.

Giorgi, A. (2012). Difficulties encountered in the application of the phenomenological method in the social sciences. Análise Psicológica, 24, 353-361. doi:10.14417/ap.175

Green, S. E. (2007). "We're tired, not sad": Benefits and burdens of mothering a child with a disability. Social Science \& Medicine, 64, 150-163. doi: 10.1016/j.socscimed.2006.08.025

Grafanaki, S. (1996). How research can change the researcher: The need for sensitivity, flexibility and ethical boundaries in conducting qualitative research in counselling/psychotherapy. British Journal of Guidance and Counselling, 24, 329-338. doi: 10.1080/03069889608253017 
Green, S. A., Ben-Sasson, A., Soto, T. W., \& Carter, A. S. (2012). Anxiety and sensory over-responsivity in toddlers with autism spectrum disorders: Bidirectional effects across time. Journal of Autism and Developmental Disorders, 42, 1112-1119. doi: 10.1007/s10803-011-1361-3

Green, J., Charman, T., Pickles, A., Wan, M. W., Elsabbagh, M., Slonims, V., ... \& Jones, E. J. (2015). Parent-mediated intervention versus no intervention for infants at high risk of autism: A parallel, single-blind, randomised trial. The Lancet Psychiatry, 2, 133-140. doi: 10.1016/S2215-0366(14)00091-1

Griffith, G. M., Hastings, R. P., Nash, S., \& Hill, C. (2010). Using matched groups to explore child behavior problems and maternal well-being in children with Down syndrome and autism. Journal of Autism and Developmental Disorders, 40, 610619. doi: 10.1007/s10803-009-0906-1

Grindle, C. F., Kovshoff, H., Hastings, R. P., \& Remington, B. (2009). Parents' experiences of home-based applied behavior analysis programs for young children with autism. Journal of Autism and Developmental Disorders, 39, 42-56. doi: $10.1007 / \mathrm{s} 10803-008-0597-\mathrm{z}$

Groenewald, T. (2004). A phenomenological research design illustrated. International Journal of Qualitative Methods, 3, 42-55. doi: 10.1177/160940690400300104

Gubrium J. F., \& Holstein J. A. (2000). Analyzing interpretive practice. In N.K. Denzin, \& Y.S. Lincoln (Eds.), Handbook of qualitative research (2nd ed.,) (pp. 487508). Thousand Oaks, CA: Sage.

Hamlyn-Wright, S., Draghi-Lorenz, R., \& Ellis, J. (2007). Locus of control fails to mediate between stress and anxiety and depression in parents of children with a developmental disorder. Autism, 11, 489-501. doi: 10.1177/1362361307083258

Haring, K. A., \& Lovett, D. L. (2010). Early intervention and early childhood services for families in rural settings. Rural Special Education Quarterly, 29, 16. doi: $10.1177 / 875687050102000302$

Harper, D. (2002). Talking about pictures: A case for photo elicitation. Visual Studies, 17, 13-26. doi: 10.1080/14725860220137345

Hart, B., \& Risley, T. R. (1995). Meaningful differences in the everyday experience of young American children. Baltimore, MD: Brookes Publishing.

Hayes, S. A., \& Watson, S. L. (2013). The impact of parenting stress: A meta-analysis of studies comparing the experience of parenting stress in parents of children with and without autism spectrum disorder. Journal of Autism and Developmental Disorders, 43, 629-642. doi: 10.1007/s10803-012-1604-y

Hoffman, C. D., Sweeney, D. P., Hodge, D., Lopez-Wagner, M. C., \& Looney, L. (2009). Parenting stress and closeness mothers of typically developing children and mothers of children with autism. Focus on Autism and Other Developmental Disabilities, 24, 178-187. doi: 10.1177/1088357609338715

Hoogsteen, L., \& Woodgate, R. L. (2013). Centering autism within the family: A qualitative approach to autism and the family. Journal of Pediatric Nursing, 28, 135-140. doi: 10.1016/j.pedn.2012.06.002

Howard, J. S., Sparkman, C. R., Cohen, H. G., Green, G., \& Stanislaw, H. (2005). A comparison of intensive behavior analytic and eclectic treatments for young 
children with autism. Research in Developmental Disabilities, 26, 359-383. doi: 10.1016/j.ridd.2004.09.005

Howlin, P., Magiati, I., \& Charman, T. (2009). Systematic review of early intensive behavioral interventions for children with autism. American Journal on Intellectual and Developmental Disabilities, 114, 23-41. doi: 10.1352/2009.114:23-41

Hunt-Jackson, J. L. (2007). Finding fathers' voices: Exploring life experiences of fathers of children with autistic spectrum disorders (Doctoral dissertation), Retrieved from: ProQuest dissertations and theses global. (304767555)

Hurtubise, K., \& Carpenter, C. (2011). Parents' experiences in role negotiation within an infant services program. Infants \& Young Children, 24, 75-86. doi: 10.1097/IYC.0b013e3182008b39

Husserl, E. (1970). The crisis of European sciences and transcendental phenomenology: An introduction to phenomenological philosophy. Evanston, IL: Northwestern University Press.

Hutton, A. M., \& Caron, S. L. (2005). Experiences of families with children with autism in rural New England. Focus on Autism and other Developmental Disabilities, 20, 180-189. doi: 10.1177/10883576050200030601

Individuals with Disabilities Education Act, 20 U.S.C. § 1400 (2004)

Ingersoll, B., \& Schreibman, L. (2006). Teaching reciprocal imitation skills to young children with autism using a naturalistic behavioral approach: Effects on language, pretend play, and joint attention. Journal of Autism and Developmental Disorders, 36, 487-505. doi: 10.1007/s10803-006-0089-y

Jackson, C. W., Traub, R. J., \& Turnbull, A. P. (2008). Parents' experiences with childhood deafness implications for family-centered services. Communication Disorders Quarterly, 29, 82-98. doi: 10.1177/1525740108314865

Johnson C.P., Myers S.M. (2007). American Academy of pediatrics council on children with disabilities: identification and evaluation of children with autism spectrum disorders. Pediatrics, (5), 1183-1215. doi: 10.1542/peds.2007-2361

Kalash, L. A., \& Olson, M. R. (2012). Perspectives of parents who have a child diagnosed with an autism spectrum disorder. Journal of the American Academy of Special Education Professionals, 93, 121.

Kalyanpur, M., Harry, B., \& Skrtic, T. (2000). Equity and advocacy expectations of culturally diverse families' participation in special education. International Journal of Disability, Development and Education, 47, 119-136. doi: 10.1080/713671106

Karst, J. S., \& Van Hecke, A. V. (2012). Parent and family impact of autism spectrum disorders: A review and proposed model for intervention evaluation. Clinical Child and Family Psychology Review, 15, 247-277. doi: 10.1007/s10567-0120119-6

Kasari, C., Freeman, S., \& Paparella, T. (2006). Joint attention and symbolic play in young children with autism: A randomized controlled intervention study. Journal of Child Psychology and Psychiatry, 47, 611-620. doi: 10.1111/j.14697610.2005.01567.x 
Kasari, C., Gulsrud, A. C., Wong, C., Kwon, S., \& Locke, J. (2010). Randomized controlled caregiver mediated joint engagement intervention for toddlers with autism. Journal of Autism and Developmental Disorders, 40, 1045-1056. doi: 10.1007/s10803-010-0955-5

Kasari, C., \& Sigman, M. (1997). Linking parental perceptions to interactions in young children with autism. Journal of Autism and Developmental Disorders, 27, 39-57. doi: 10.1023/A:1025869105208

Keenan, M., Dillenburger, K., Doherty, A., Byrne, J. \& Gallagher, S. (2010). Experiences of parents during diagnosis and forward planning for children with autism spectrum disorder. Journal of Applied Research in Intellectual Disabilities, 23, 390-397. doi: 10.1111/j.1468-3148.2010.00555.x

Keilty, B. (Ed.). (2010). The early intervention guidebook for families and professionals: Partnering for success. New York, NY: Teachers College Press.

Ketelaar, M., Vermeer, A., Helders, P. J., \& Hart, H. T. (1998). Parental participation in intervention programs for children with cerebral palsy: A review of research. Topics in Early Childhood Special Education, 18, 108-117. doi: 10.1177/027112149801800206

Kim, S. H., Macari, S., Koller, J., \& Chawarska, K. (2016). Examining the phenotypic heterogeneity of early autism spectrum disorder: Subtypes and short-term outcomes. Journal of Child Psychology and Psychiatry, 57, 93-102. doi: $10.1111 /$ jcpp. 12448

King, G. A., Zwaigenbaum, L., King, S., Baxter, D., Rosenbaum, P., \& Bates, A. (2006). A qualitative investigation of changes in the belief systems of families of children with autism or Down syndrome. Child Care, Health and Development, 32, 353369. doi: 10.1111/j.1365-2214.2006.00571.x

Koegel, L. K., Koegel, R. L., Harrower, J. K., \& Carter, C. M. (1999). Pivotal response intervention I: Overview of approach. Research and Practice for Persons with Severe Disabilities, 24, 174-185. doi: 10.2511/rpsd.24.3.174

Landa, R., \& Garrett-Mayer, E. (2006). Development in infants with autism spectrum disorders: A prospective study. Journal of Child Psychology and Psychiatry, 47, 629-638. doi: 10.1111/j.1469-7610.2006.01531.x

Landa, R. J., Gross, A. L., Stuart, E. A., \& Faherty, A. (2013). Developmental trajectories in children with and without autism spectrum disorders: The first 3 years. Child Development, 84, 429-442. doi: 10.1111/j.1467-8624.2012.01870.x

Landgren, K., \& Hallström, I. (2011). Parents' experience of living with a baby with infantile colic-A phenomenological hermeneutic study. Scandinavian Journal of Caring Sciences, 25, 317-324. doi: 10.1111/j.1471-6712.2010.00829.x

Landry, S. H., Smith, K. E., \& Swank, P. R. (2006). Responsive parenting: Establishing early foundations for social, communication, and independent problem-solving skills. Developmental Psychology, 42, 627. doi: 10.1037/0012-1649.42.4.627

LaRossa, R., \& Wolf, J. H. (1985). On qualitative family research. Journal of Marriage and the Family, 531-541. doi: 10.1111/j.1741-3737.2005.00179.x

Latta, A., Rampton, T., Rosemann, J., Peterson, M., Mandleco, B., Dyches, T., \& Roper, S. (2014). Snapshots reflecting the lives of siblings of children with autism 
spectrum disorders. Child Care, Health and Development, 40, 515-524. doi: $10.1111 /$ cch. 12100

Lefkowitz, D. S., Baxt, C., \& Evans, J. R. (2010). Prevalence and correlates of posttraumatic stress and postpartum depression in parents of infants in the neonatal intensive care unit (NICU). Journal of Clinical Psychology in Medical Settings, 17, 230-237 doi: 10.1007/s10880-010-9202-7

Lester, S. (1999). An introduction to phenomenological research. Retrieved from: https://www.researchgate.net/publication/255647619_An_introduction_to_pheno menological research.

Lincoln, Y. S., \& Guba, E. G. (1985). Naturalistic inquiry (Vol. 75). Beverly Hills, CA: Sage.

Lindgren, S., \& Doobay, A. (2011). Evidence-based interventions for autism spectrum disorders. Retrieved from: https://pdfs.semanticscholar.org/5a39/5f50b01b60f8e0b03567acf95f9eb831bc03. pdf

Linton, S. (1998). Claiming disability: Knowledge and identity. New York, NY: University Press.

Loeffler, T. A. (2005). Looking deeply in: Using photo-elicitation to explore the meanings of outdoor education experiences. The Journal of Experiential Education, 27, 343.

Lord, C., \& McGee, J. P. (Eds.) (2001). Educating children with autism: Committee on educational interventions for children with autism. Washington, DC: National Academy Press.

Lord C, Rutter M, DiLavore PC, Risi S. (1999) Autism Diagnostic Observation Schedule (ADOS) [Measurement Instrument]. Los Angeles, CA: Western Psychological Services.

Lovaas, O.I. (1987). Behavioral treatment and normal educational and intellectual functioning in young autistic children. Journal of Consulting and Clinical Psychology, 55, 3-9. doi: 10.1037/0022-006X.55.1.3

Lutz, H. R., Patterson, B. J., \& Klein, J. (2012). Coping with autism: A journey toward adaptation. Journal of Pediatric Nursing, 27, 206-213. doi: 10.1016/j.pedn.2011.03.013

Maestro, S., Muratori, F., Cavallaro, M. C., Pei, F., Stern, D., Golse, B., \& PalacioEspasa, F. (2002). Attentional skills during the first 6 months of age in autism spectrum disorder. Journal of the American Academy of Child \& Adolescent Psychiatry, 41, 1239-1245. doi: 10.1097/00004583-200210000-00014

Mesibov, G. B., Shea, V., \& Schopler, E. (2005). The TEACCH approach to autism spectrum disorders. New York, NY: Springer Science \& Business Media.

Mahoney, G., Boyce, G., Fewell, R. R., Spiker, D., \& Wheeden, C. A. (1998). The relationship of parent-child interaction to the effectiveness of early intervention services for at-risk children and children with disabilities. Topics in Early Childhood Special Education, 18, 5-17. doi: 10.1177/027112149801800104

Mandleco, B. (2013). Research with children as participants: Photo elicitation. Journal for Specialists in Pediatric Nursing, 18, 78-82. doi: 10.1111/jspn.12012 
Mandell, D., Ittenbach, R. F., Levy, S. E., \& Pinto-Martin, J. A. (2007). Disparities in diagnoses received prior to a diagnosis of autism spectrum disorder. Journal of Autism and Developmental Disorders, 37, 1795-1802. doi:10.1007/s10803-0060314-8

Mandell, D. S., Listerud, J., Levy, S. E., \& Pinto-Martin, J. A. (2002). Race differences in the age at diagnosis among Medicaid-eligible children with autism. Journal of the American Academy of Child \& Adolescent Psychiatry, 41, 1447-1453. doi: 10.1097/00004583-200212000-00016

Mandell, D. S., Novak, M. M., \& Zubritsky, C. D. (2005). Factors associated with age of diagnosis among children with autism spectrum disorders. Pediatrics, 116, 14801486. doi: 10.1542/peds.2005-0185

Mandell, D. S., Wiggins, L. D., Carpenter, L. A., Daniels, J., DiGuiseppi, C., Durkin, M. S., ... \& Shattuck, P. T. (2009). Racial/ethnic disparities in the identification of children with autism spectrum disorders. American Journal of Public Health, 99, 493-498. doi: 10.2105/AJPH.2007.131243

Marfo, K. (1991). The maternal directiveness theme in mother-child interaction research: Implications for early intervention. In K. Marfo (Ed.), Early intervention in transition: Current perspectives on programs for handicapped infants (pp. 177203). New York, NY: Praege.

McGrath, J. M. (2001). Building relationships with families in the NICU: Exploring the guarded alliance. The Journal of perinatal \& neonatal nursing, 15, 74-83. doi: 10.1097/00005237-200112000-00007

McWilliam, R. A., Casey, A. M., \& Sims, J. (2009). The routines-based interview: A method for gathering information and assessing needs. Infants \& Young Children, 22, 224-233. doi: 10.1097/IYC.0b013e3181abe1dd

Meadan, H., Stoner, J. B., \& Angell, M. E. (2010). Review of literature related to the social, emotional, and behavioral adjustment of siblings of individuals with autism spectrum disorder. Journal of Developmental and Physical Disabilities, 22, 83-100. doi: 10.1007/s10882-009-9171-7

Meirsschaut, M., Roeyers, H., \& Warreyn, P. (2010). Parenting in families with a child with autism spectrum disorder and a typically developing child: Mothers' experiences and cognitions. Research in Autism Spectrum Disorders, 4, 661-669. doi: 10.1016/j.rasd.2010.01.002

Merriam, S.B. (2009). Qualitative research: A guide to design and implementation. San Francisco, CA: Jossey-Bass.

Mishna, F., Antle, B. J., \& Regehr, C. (2004). Tapping the perspectives of children: Emerging ethical issues in qualitative research. Qualitative Social Work, 3, 449468. doi: $10.1177 / 1473325004048025$

Mok, E., \& Leung, S. F. (2006). Nurses as providers of support for mothers of premature infants. Journal of Clinical Nursing, 15, 726-734. doi: 10.1111/j.13652702.2006.01359.x

Murdoch, M. R., \& Franck, L. S. (2012). Gaining confidence and perspective: A phenomenological study of mothers' lived experiences caring for infants at home 
after neonatal unit discharge. Journal of Advanced Nursing, 68, 2008-2020. doi: 10.1111/j.1365-2648.2011.05891.x

Murphy, D. L., Lee, I.M., Turnbull, A. P., \& Turnbull, V. (1995). The family-centered program rating scale an instrument for program evaluation and change. Journal of Early Intervention, 19, 24-42. doi: 10.1177/105381519501900104

National Research Council. (2001). Educating children with autism. Washington, DC: National Academy Press.

Neely-Barnes, S. L., Hall, H. R., Roberts, R. J. \& Graff, J. C. (2011). Parenting a child with an autism spectrum disorder: Public perceptions and parental conceptualizations. Journal of Family Social Work, 14, 208-225. doi: 10.1080/10522158.2011.571539

Nelson, A. M. (2002). A metasynthesis: Mothering other-than-normal children. Qualitative Health Research, 12, 515-530. doi: $10.1177 / 104973202129120043$

Nicolaou, M., Rosewell, R., Marlow, N., \& Glazebrook, C. (2009). Mothers' experiences of interacting with their premature infants. Journal of Reproductive and Infant Psychology, 27, 182-194. doi: 10.1080/02646830801922796

Noon, N., \& McCormick, L. (1993). Early intervention in a natural environment. Pacific Grove, CA: Brooks/Cole.

Odom, S. L., Brantlinger, E., Gersten, R., Horner, R. H., Thompson, B., \& Harris, K. R. (2005). Research in special education: Scientific methods and evidence-based practices. Exceptional children, 71, 137-148. doi: 10.1177/001440290507100201

Odom, S. L., Collet-Klingenberg, L., Rogers, S. J., \& Hatton, D. D. (2010). Evidencebased practices in interventions for children and youth with autism spectrum disorders. Preventing School Failure: Alternative Education for Children and yYouth, 54, 275-282. doi: 10.1080/10459881003785506

Onwuegbuzie, A. J., \& Leech, N. L. (2007). Validity and qualitative research: An oxymoron? Quality \& Quantity, 41, 233-249. doi: 10.1007/s11135-006-9000-3

Oregon Department of Education (2015). Oregon State Interagency Coordinating council early intervention / early childhood special education: Annual report. 2014-2015. Retrieved from: http://www.oregon.gov/ode/students-andfamily/SpecialEducation/GeneralSupervision/Documents/2016siccgovreport.pdf

Osborne, L. A., McHugh, L., Saunders, J., \& Reed, P. (2008). Parenting stress reduces the effectiveness of early teaching interventions for autistic spectrum disorders. Journal of Autism and Developmental Disorders, 38, 1092-1103. doi: 0.1007/s10803-007-0497-7

Osborne, L. A., \& Reed, P. (2008). Parents' perceptions of communication with professionals during the diagnosis of autism. Autism, 12, 309-324. doi: $10.1177 / 1362361307089517$

Osterling, J., \& Dawson, G. (1994). Early recognition of children with autism: A study of first birthday home videotapes. Journal of Autism and Developmental Disorders, 24, 247-257. doi: 10.1007/BF02172225 
Osterling, J. A., Dawson, G., \& Munson, J. A. (2002). Early recognition of 1-year-old infants with autism spectrum disorder versus mental retardation. Development and Psychopathology, 14, 239-251. doi: 10.1017/S0954579402002031

Osterling, I., Visser, J., Swinkels, S., Rommelse, N., Donders, R., Woudenberg, T., ... \& Buitelaar, J. (2010). Randomized controlled trial of the focus parent training for toddlers with autism: 1-year outcome. Journal of Autism and Developmental Disorders, 40, 1447-1458. doi: 10.1007/s10803-010-1004-0

Ozonoff, S., Young, G. S., Carter, A., Messinger, D., Yirmiya, N., Zwaigenbaum, L., ... $\&$ Hutman, T. (2011). Recurrence risk for autism spectrum disorders: a Baby Siblings Research Consortium study. Pediatrics, 128, 488-495. doi: 10.1542/peds.2010-2825

Ozonoff, S., Young, G. S., Steinfeld, M. B., Hill, M. M., Cook, I., Hutman, T., ... \& Sigman, M. (2009). How early do parent concerns predict later autism diagnosis? Journal of Developmental and Behavioral Pediatrics, 30, 367-375. Retrieved from: https://www-ncbi-nlm-nihgov.proxy.lib.pdx.edu/pmc/articles/PMC2919345/

Patterson, S. Y., \& Smith, V. (2011). The experience of parents of toddlers diagnosed with autism spectrum disorder in the More Than Words parent education program. Infants \& Young Children, 24, 329-343. doi: 10.1097/IYC.0b013e31822c10e4

Patton, M.Q. (1990). Qualitative evaluation and research methods (2nd ed.). Newbury Park, CA: Sage.

Peterson, C. A., Luze, G. J., Eshbaugh, E. M., Jeon, H. J., \& Kantz, K. R. (2007). Enhancing parent-child interactions through home visiting: Promising practice or unfulfilled promise? Journal of Early Intervention, 29, 119-140. doi: $10.1177 / 105381510702900205$

Perryman, T. Y., Carter, A. S., Messinger, D. S., Stone, W. L., Ivanescu, A. E., \& Yoder, P. J. (2013). Brief report: Parental child-directed speech as a predictor of receptive language in children with autism symptomatology. Journal of Autism and Developmental Disorders, 43, 1983-1987. doi: 10.1007/s10803-012-1725-3

Phelps, K. W., Hodgson, J. L., McCammon, S. L., \& Lamson, A. L. (2009). Caring for an individual with autism disorder: A qualitative analysis. Journal of Intellectual and Developmental Disability, 34, 27-35. doi: 10.1080/13668250802690930

Phelps, K. W., McCammon, S. L., Wuensch, K. L., \& Golden, J. A. (2009). Enrichment, stress, and growth from parenting an individual with an autism spectrum disorder. Journal of Intellectual and Developmental Disability, 34, 133-141. doi: 10.1080/13668250902845236

Phillips, D. A., \& Shonkoff, J. P. (Eds.). (2000). From neurons to neighborhoods: The science of early childhood development. Washington, DC: National Academies Press.

Piaget, J. (1929 / 1960). The child's conception of the world. London: Routlege \& Kegan Press.

Presmanes, A. G., Walden, T. A., Stone, W. L., \& Yoder, P. J. (2007). Effects of different attentional cues on responding to joint attention in younger siblings of children 
with autism spectrum disorders. Journal of Autism and Developmental Disorders, 37, 133-144. doi: 10.1007/s10803-006-0338-0

Prizant, B. M., Wetherby, A. M., Rubin, E., \& Laurent, A. C. (2003). The SCERTS Model: A transactional, family-centered approach to enhancing communication and socioemotional abilities of children with autism spectrum disorder. Infants \& Young Children, 16, 296-316. Retrieved from: http://ovidsp.tx.ovid.com.proxy.lib.pdx.edu/sp3.26.1a/ovidweb.cgi?\&S=KNNOFPHMFJDDAFNINCGKPALBKIBAAA00\&Li $\mathrm{nk}+$ Set $=$ S.sh. $22 \% 7 \mathrm{c} 1 \%$ csl 10

Rao, P. A., Raman, V., Thomas, T., \& Ashok, M. V. (2015). IQ in autism: Is there an alternative global cognitive index? Indian Journal of Psychological Medicine, 37, 48. doi: $10.4103 / 0253-7176.150819$

Reed, P., \& Osborne, L. A. (2014). Parenting and autism spectrum disorders. In V. Patel, V.R. Preedy, \& C.R. Martin (Eds.), Comprehensive guide to autism (pp. 185206). New York, NY: Springer.

Rehm, R. S., \& Bisgaard, R. (2008). Transition to home from the neonatal intensive care unit. Journal of Pediatric Nursing, 23, 20-21. doi: 10.1016/j.pedn.2008.01.066

Reichow, B., \& Wolery, M. (2009). Comprehensive synthesis of early intensive behavioral interventions for young children with autism based on the UCLA young autism project model. Journal of Autism and Developmental Disorders, 39, 23-41. doi: 10.1007/s10803-008-0596-0

Reiners, G. M. (2012). Understanding the differences between Husserl's (descriptive) and Heidegger's (interpretive) phenomenological research. Journal of Nursing \& Care, 1, 1-6. doi: 10.4172/2167-1168.1000119

Rhodes, S. (2013). Theories of child development [PowerPoint Slides]. Retrieved from http://uoit.blackboard.com.

Robertson, S. M. (2009). Neurodiversity, quality of life, and autistic adults: Shifting research and professional focuses onto real-life challenges. Disability Studies Quarterly, 30. .n.p. doi: 10.18061/dsq.v30i1.1069

Rodger, S., Keen, D., Braithwaite, M., \& Cook, S. (2008). Mothers' satisfaction with a home based early intervention programme for children with ASD. Journal of Applied Research in Intellectual Disabilities, 21, 174-182. doi: 10.1111/j.14683148.2007.00393.x

Rogers, S. J. (2004). Developmental regression in autism spectrum disorders. Mental Retardation and Developmental Disabilities Research Reviews, 10, 139-143. doi: 10.1002/mrdd.20027

Rogers, S. J. (2009). What are infant siblings teaching us about autism in infancy? Autism Research ,2, 125-137. doi: 10.1002/aur.81

Rogers, S.J. \& Dawson, G. (2010) Early Start Denver Model for Young Children with Autism: Promoting language, learning and engagement. New York, NY: Guilford Press.

Rogers, S. J., \& DiLalla, D. L. (1990). Age of symptom onset in young children with pervasive developmental disorders. Journal of the American Academy of Child \& Adolescent Psychiatry, 29, 863-872. doi:10.1097/00004583-199011000-00004 
Rogers, S. J., Estes, A., Lord, C., Vismara, L., Winter, J., Fitzpatrick, A., ... \& Dawson, G. (2012). Effects of a brief Early Start Denver Model (ESDM)-based parent intervention on toddlers at risk for autism spectrum disorders: A randomized controlled trial. Journal of the American Academy of Child \& Adolescent Psychiatry, 51, 1052-1065. doi: 10.1016/j.jaac.2012.08.003

Rogers, S.J. \& Vismara, L.A. (2014). Interventions for infants and toddlers at risk for autism spectrum disorder. In F.R. Volkmar, S.J. Rogers, R. Paul, \& K.A. Pelphrey (Eds.), Handbook of autism and pervasive developmental disorders, $\left(4^{\text {th }} \mathrm{Ed}\right.$.) (pp. 739-770). New York, NY: John Wiley \& Sons.

Rondeau, E., Klein, L. S., Masse, A., Bodeau, N., Cohen, D., \& Guilé, J. M. (2011). Is pervasive developmental disorder not otherwise specified less stable than autistic disorder? A meta-analysis. Journal of Autism and Developmental Disorders, 41, 1267-1276. doi: 10.1007/s10803-010-1155-Z

Rosemann, J., Palicharla, A., Rampton, T., Dyches, T., \& Freeborn, D. (2015). Sibling snapshots: Living with youth who have autism or Down syndrome. International Journal of Integrative Pediatrics and Environmental Medicine, 2, 13-30. doi: 10.6000\%2FIjipem\%2F $2015 \% 2 \mathrm{~F} 3$

Rozga, A., Hutman, T., Young, G. S., Rogers, S. J., Ozonoff, S., Dapretto, M., \& Sigman, M. (2011). Behavioral profiles of affected and unaffected siblings of children with autism: Contribution of measures of mother-infant interaction and nonverbal communication. Journal of Autism and Developmental Disorders, 41, 287-301. doi: 10.1007/s10803-010-1051-6

Ryan, S., \& Runswick - Cole, K. (2008). Repositioning mothers: Mothers, disabled children and disability studies. Disability \& Society, 23, 199-210. doi: $10.1080 / 09687590801953937$

Saint-Georges, C., Cassel, R. S., Cohen, D., Chetouani, M., Laznik, M. C., Maestro, S., \& Muratori, F. (2010). What studies of family home movies can teach us about autistic infants: A literature review. Research in Autism Spectrum Disorders, 4, 355-366. doi: 10.1016/j.rasd.2009.10.017

Salvatore, S., \& Valsiner, J. (2010). Between the general and the unique: Overcoming the nomothetic versus idiographic opposition. Theory \& Psychology, 20, 817-833. doi: $10.1177 / 0959354310381156$

Sameroff, A. J., Seifer, R., Baldwin, A., \& Baldwin, C. (1993). Stability of intelligence from preschool to adolescence: The influence of social and family risk factors. Child Development, 64, 80-97. doi: 10.1111/j.1467-8624.1993.tb02896.x

Sameroff, A. J., Seifer, R., Barocas, R., Zax, M., \& Greenspan, S. (1987). Intelligence quotient scores of 4-year-old children: Social-environmental risk factors. Pediatrics, 79 (3), 343-350.

Sandall, S., Hemmeter, M. L., Smith, B. J., \& McLean, M. E. (2005). DEC recommended practices: A comprehensive guide. Longmont, $\mathrm{CO}$ : Sopris West.

Sandall, S. R., Smith, B. J., Mclean, M. E., \& Ramsey, A. B. (2002). Qualitative research in early intervention/early childhood special education. Journal of Early Intervention, 25, 129-136. doi: 10.1177/105381510202500210 
Schertz, H. H., Odom, S. L., Baggett, K. M., \& Sideris, J. H. (2013). Effects of joint attention mediated learning for toddlers with autism spectrum disorders: An initial randomized controlled study. Early Childhood Research Quarterly, 28, 249-258. doi: 10.1016/j.ecresq.2012.06.006

Schieve, L. A., Blumberg, S. J., Rice, C., Visser, S. N., \& Boyle, C. (2007). The relationship between autism and parenting stress. Pediatrics, 119, 114-121. doi: 10.1542/peds.2006-2089Q

Schreibman, L. (2000). Intensive behavioral/psychoeducational treatments for autism: Research needs and future directions. Journal of Autism and Developmental Disorders, 30, 373-378. doi: 10.1023/A:1005535120023

Schreibman, L., Dawson, G., Stahmer, A. C., Landa, R., Rogers, S. J., McGee, G. G., ... \& McNerney, E. (2015). Naturalistic developmental behavioral interventions: Empirically validated treatments for autism spectrum disorder. Journal of Autism and Developmental Disorders, 45, 1-18. doi: 10.1007/s10803-015-2407-8

Schultz, R. T. (2005). Developmental deficits in social perception in autism: The role of the amygdala and fusiform face area. International Journal of Developmental Neuroscience, 23, 125-141. doi: 10.1016/j.ijdevneu.2004.12.012

Seidman, I. (2013). Interviewing as qualitative research: A guide for researchers in education and the social sciences $\left(4^{\text {th }}\right.$ ed). New York, NY: Teachers College Press.

Seligman M., \& Darling R. B. (2007). Ordinary families, special children; A systems approach to childhood disability. New York, NY: The Guildford Press.

Shakespeare, T. (2013). Disability rights and wrongs revisited. New York, NY: Routledge.

Shannon, P. (2004). Barriers to family-centered services for infants and toddlers with developmental delays. Social Work, 49, 301-308. doi: 10.1093/sw/49.2.301

Shattuck, P. T., Durkin, M., Maenner, M., Newschaffer, C., Mandell, D. S., Wiggins, L., ... \& Baio, J. (2009). Timing of identification among children with an autism spectrum disorder: Findings from a population-based surveillance study. Journal of the American Academy of Child \& Adolescent Psychiatry, 48, 474-483. doi: 10.1097/CHI.0b013e31819b3848

Shaw, R. (2001). Why use interpretative phenomenological analysis in health psychology? Health Psychology Update, 10, 48-52.

Shonkoff, J. P., \& Hauser-Cram, P. (1987). Early intervention for disabled infants and their families: a quantitative analysis. Pediatrics, 80, 650-658. Retrieved from: http://interventie-timpurie.ssbn.ro/wp content/uploads/2016/01/EarlyInterventionforDisabledInfantsandtheirFamiliesAQ uantitativeAnalysis.pdf

Siller, M., \& Sigman, M. (2002). The behaviors of parents of children with autism predict the subsequent development of their children's communication. Journal of Autism and Developmental Disorders, 32, 77-89. doi: 10.1023/A:1014884404276

Sokolowski, R. (2000). Introduction to phenomenology. London: Cambridge University Press. 
Sperry, L. A., Whaley, K. T., Shaw, E., \& Brame, K. (1999). Services for Young Children with Autism Spectrum Disorder: Voices of Parents and Providers. Infants \& Young Children, 11, 17-33.

Stahmer A.C., \& Gist K. (2001). The effects of an accelerated parent education program on technique mastery and child outcome. Journal of Positive Behavioral Intervention 3, 75-82. doi: 10.1177/109830070100300203

Stahmer, A. C., \& Ingersoll, B. (2004). Inclusive programming for toddlers with autism spectrum disorders: Outcomes from the children's toddler school. Journal of Positive Behavior Interventions, 6, 67-82. doi: 10.1177/10983007040060020201

Stahmer, A. C., \& Mandell, D. S. (2007). State infant/toddler program policies for eligibility and services provision for young children with autism. Administration and Policy in Mental Health and Mental Health Services Research, 34, 29-37. doi: 0.1007/s10488-006-0060-4

Stake, R. E. (1995). The art of case study research. Thousand Oaks, CA: Sage.

Steege, M. W., Mace, F. C., Perry, L., \& Longenecker, H. (2007). Applied behavior analysis: Beyond discrete trial teaching. Psychology in the Schools, 44, 91-99. doi: $10.1002 /$ pits. 20208

Steiner, A. M., Gengoux, G. W., Klin, A., \& Chawarska, K. (2013). Pivotal response treatment for infants at-risk for autism spectrum disorders: A pilot study. Journal of Autism and Developmental Disorders, 43, 91-102. doi: 10.1007/s10803-012$1542-8$

Stone, W. L., \& Lemanek, K. L. (1990). Parental report of social behaviors in autistic preschoolers. Journal of Autism and Developmental Disorders, 20, 513-522. doi: 10.1007/BF02216056

Stone, W. L., \& Rosenbaum, J. L. (1988). A comparison of teacher and parent views of autism. Journal of Autism and Developmental Disorders, 18, 403-414. doi: 10.1007/BF02212195

Strauss, A., \& Corbin, J. (1994). Grounded theory methodology. In Handbook of qualitative research (chapter 17). Retrieved from: http://www.depts.ttu.edu/education/ourpeople/Faculty/additional_pages/duemer/epsy_5382_class_materials/Groundedtheory-methodology.pdf

Stuart, M., \& McGrew, J. H. (2009). Caregiver burden after receiving a diagnosis of an autism spectrum disorder. Research in Autism Spectrum Disorders, 3, 86-97. doi: 10.1016/j.rasd.2008.04.006

Swanson-Kauffman, K.M., \& Schonwald, E. (1988). Phenomenology. In B. Sater (Ed.). Paths to knowledge: Innovative research methods for nursing. (pp. 97-105). New York, NY: National League for Nursing.

Tamis-LeMonda, C. S., Bornstein, M. H., \& Baumwell, L. (2001). Maternal responsiveness and children's achievement of language milestones. Child Development, 72, 748-767. doi: 10.1111/1467-8624.00313

Trivette, C. M., Dunst, C. J., \& Hamby, D. W. (2010). Influences of family-systems intervention practices on parent-child interactions and child development. Topics in Early Childhood Special Education, 30, 3-19. doi: 10.1177/0271121410364250 
U.S. Department of Education. (1999). Part II: Assistance to states for the education of children with disabilities and the early intervention program for infants and toddlers with disabilities; Final regulations. 64 FR 12406. 34 CFR $\S \S 300,303$.

U.S. Department of Education (2016). Non-regulatory guidance: Using evidence to strengthen education investment. Retrieved from:

https://www2.ed.gov/policy/elsec/leg/essa/guidanceuseseinvestment.pdf.

Vagle, M. D. (2014). Crafting phenomenological research. Walnut Creek, CA: Left Coast Press.

Van Auken, P. M., Frisvoll, S. J., \& Stewart, S. I. (2010). Visualising community: Using participant-driven photo-elicitation for research and application. Local Environment, 15, 373-388. doi: 10.1080/13549831003677670

Van Manen (1997). Researching lived experiences: Human science for an action sensitive pedagogy ( $2^{\text {nd }}$ ed.). Walnut Creek, CA: Left Coast Press.

Vernon, T. W., Koegel, R. L., Dauterman, H., \& Stolen, K. (2012). An early social engagement intervention for young children with autism and their parents. Journal of Autism and Developmental Disorders, 42, 2702-2717. doi: 10.1007/s10803-012-1535-7

Vismara, L. A., Colombi, C., \& Rogers, S. J. (2009). Can one hour per week of therapy lead to lasting changes in young children with autism? Autism, 13, 93-115. doi: $10.1177 / 1362361307098516$

Volkmar, F. R., Cicchetti, D. V., Bregman, J., \& Cohen, D. J. (1992). Three diagnostic systems for autism: DSM-III, DSM-III-R, and ICD-10. Journal of Autism and Developmental Disorders, 22, 483-492. doi: 10.1007/BF01046323

Wade, C. M., Mildon, R. L., \& Matthews, J. M. (2007). Service delivery to parents with an intellectual disability: Family-centred or professionally centred? Journal of Applied Research in Intellectual Disabilities, 20, 87-98. doi: 10.1111/j.14683148.2005.00297.x

Wagner, A. L., Wallace, K. S., \& Rogers, S. J. (2014). Developmental approaches to treatment of young children with autism spectrum disorder. In J. Tarbox, D.R. Discon, P. Sturmey, J.L. Matson (Eds.). Handbook of early intervention for autism spectrum disorders (pp. 393-427). New York, NY: Springer.

Wan, M. W., Green, J., Elsabbagh, M., Johnson, M., Charman, T., Plummer, F., \& BASIS Team. (2012). Parent-infant interaction in infant siblings at risk of autism. Research in Developmental Disabilities, 33, 924-932. doi: 10.1016/j.ridd.2011.12.011

Wan, M. W., Green, J., Elsabbagh, M., Johnson, M., Charman, T., \& Plummer, F. (2013). Quality of interaction between at-risk infants and caregiver at 12-15 months is associated with 3-year autism outcome. Journal of Child Psychology and Psychiatry, 54, 763-771. doi: 10.1111/jcpp.12032

Warren, S. F., \& Kaiser, A. P. (1986). Incidental language teaching: A critical review. Journal of Speech and Hearing Disorders, 51, 291-299. doi: 10.1044/jshd.5104.291

Warren, Z., McPheeters, M. L., Sathe, N., Foss-Feig, J. H., Glasser, A., \& VeenstraVanderWeele, J. (2011). A systematic review of early intensive intervention for 
autism spectrum disorders. Pediatrics, 127, 1303-1311. doi: 10.1542/peds.20110426

Watson, J., (2011). Nursing. Boulder, CO: University Press of Colorado.

Webster, A., Feiler, A., Webster, V., \& Lovell, C. (2004). Parental perspectives on early intensive intervention for children diagnosed with autistic spectrum disorder. Journal of Early Childhood Research, 2, 25-49. doi: 10.1177/1476718X0421002

Weisner, T. S. (1996). Why ethnography should be the most important method in the study of human development. In R. Jessor, A. Colby, \& R.A. Shweder (Eds.), (305-324). Chicago, IL: University of Chicago Press.

Wetherby, A. M., Guthrie, W., Woods, J., Schatschneider, C., Holland, R. D., Morgan, L., \& Lord, C. (2014). Parent-implemented social intervention for toddlers with autism: An RCT. Pediatrics, 134, 1084-1093. doi: 10.1542/peds.2014-0757

Wetherby, A. M., \& Woods, J. J. (2006). Early social interaction project for children with autism spectrum disorders beginning in the second year of life: A preliminary study. Topics in Early Childhood Special Education, 26, 67-82. doi: 10.1177/02711214060260020201

Wigham, S., Rodgers, J., South, M., McConachie, H., \& Freeston, M. (2015). The interplay between sensory processing abnormalities, intolerance of uncertainty, anxiety and restricted and repetitive behaviours in autism spectrum disorder. Journal of Autism and Developmental Disorders, 45, 943-952. doi: 10.1007/s 10803-014-2248-x

Wimpory, D. C., Hobson, R. P., Williams, J. M. G., \& Nash, S. (2000). Are infants with autism socially engaged? A study of recent retrospective parental reports. Journal of Autism and Developmental Disorders, 30, 525-536. doi: 10.1023/A:1005683209438

Wojnar, D.M. \& Swanson, K.M. (2007). Phenomenology as an exploration. Journal of Holistic Nursing, 25, 172-180. doi: 10.1177/0898010106295172

Woods, J. J., \& Brown, J. A. (2011). Integrating family capacity-building and child outcomes to support social communication development in young children with autism spectrum disorder. Topics in Language Disorders, 31, 235-246. doi: 10.1097/TLD.0b013e318227fde4

Wong, C., Odom, S. L., Hume, K. A., Cox, A. W., Fettig, A., Kucharczyk, S., ... \& Schultz, T. R. (2015). Evidence-based practices for children, youth, and young adults with autism spectrum disorder: A comprehensive review. Journal of Autism and Developmental Disorders, 45(7), 1951-1966. doi: 10.1007/s10803-014-2351$\mathrm{Z}$

Yamada, A., Suzuki, M., Kato, M., Suzuki, M., Tanaka, S., Shindo, T., ... \& Furukawa, T. A. (2007). Emotional distress and its correlates among parents of children with pervasive developmental disorders. Psychiatry and Clinical Neurosciences, 61, 651-657. doi: 10.1111/j.1440-1819.2007.01736.x

Yin, R.K. (2014). Case study research: Design and methods. Los Angeles, CA: Sage. Yirmiya, N., Gamliel, I., Pilowsky, T., Feldman, R., Baron-Cohen, S., \& Sigman, M. (2006). The development of siblings of children with autism at 4 and 14 months: 
Social engagement, communication, and cognition. Journal of Child Psychology and Psychiatry, 47, 511-523. doi: 10.1111/j.1469-7610.2005.01528.x

Zablotsky, B., Colpe, L. J., Pringle, B. A., Kogan, M. D., Rice, C., \& Blumberg, S. J. (2017). Age of parental concern, diagnosis, and service initiation among children with autism spectrum disorder. American Journal on Intellectual and Developmental Disabilities, 122, 49-61. doi: 0.1352/1944-7558-122.1.49

Zwaigenbaum, L., Bauman, M. L., Choueiri, R., Fein, D., Kasari, C., Pierce, K., ... \& McPartland, J. C. (2015). Early identification and interventions for autism spectrum disorder: Executive summary. Pediatrics, 136 (Supplement 1), 1-9. doi: 10.1542/peds.2014-3667B

Zwaigenbaum, L., Bryson, S., Rogers, T., Roberts, W., Brian, J., \& Szatmari, P. (2005). Behavioral manifestations of autism in the first year of life. International Journal of Developmental Neuroscience, 23, 143-152. doi:

10.1016/j.ijdevneu.2004.05.001 


\section{APPENDIX A}

\section{RECRUITMENT MATERIALS FOR SNOWBALL SAMPLE}

This phone script or email will be used with Education Service District managers, personnel, or referred contacts to share information about the study to potential participants. The participants will be sent an email (Appendix B) or a flyer (Appendix C). Interested participants will then contact the researcher (me) and I will conduct an initial phone screen (Appendix D).

\section{PHONE SCRIPT}

Hello, my name is Donna Barrow and I'm a doctoral student at Portland State University. I am contacting you to talk about a research study I'm conducting with parents with young children with autism. I will be conducting a qualitative study to talk with parents about their experiences as a parent receiving early intervention services. The study will involve two interviews and my goal is to understand the unique experience parenting a young child with autism.

I am looking for 5-7 parents who have a child between 24 and 36 months and who are receiving early intervention services for autism.

I will be recruiting families by distributing information about the study to potential parent participants. I have a flyer and an email that contacts that you can share with families. Families who are interested can contact me for more information and an initial phone screen to determine if they meet the criteria for the study.

Would it be all right if I sent you more information about my study and follow up with you next week to talk more about recruiting participants who may be interested?

\section{EMJL TO ESD'S OR OTHER CONTACTS}

Dear

My name is Donna Barrow and I am a student at PSU pursuing my doctoral degree. I am writing to you to see if you would be willing help me recruit participants for my research study. I have attached a flyer to this email with more detail about the study.

I will be conducting a qualitative study to talk with parents about their experiences as a parent receiving early intervention services. The study will involve two interviews and my goal is to understand the unique experience parenting a young child with autism. I am looking for 5-7 parents who have a child between 24 and 36 months and who are receiving early intervention services for autism.

I am contacting Education Service Districts to see if they would be willing to distribute the attached flyer to families or staff who work with parents of young children with autism. The potential parent participants may contact me if they are interested in 
participating in the interview. I also have an email with information that can be sent out to families. Families who are interested can contact me for more information and an initial phone screen to determine if they meet the criteria for the study.

The overall goal of this project to is to gain insight into the caregiving experiences of parents of infants and toddlers with autism who are receiving early intervention services. My study will involve two unstructured interviews. I am interested in talking parents with an infant or toddler with autism (between the ages of 24 and 36 months). My study will involve two conversations with parents about 'what it means to be a parent of a very young child with autism' and your experience with early intervention services.

I will call you next week to follow up on this email. You may also contact me at 503756-7006 if you'd like to talk before then.

Thank you,

Donna Barrow, M.S., Ed.D. Candidate 


\section{APPENDIX B}

\section{RECRUITMENT EMAIL TO POTENTIAL PARTICIPANT}

This email will be forwarded to families by the recruitment contact (i.e., ESD staff, families referring to other families, other individuals who know of a potential participant). If families are interested they will contact the researcher (me) to find out more information and participate in an initial screening for the study.

\section{Dear Parent,}

My name is Donna Barrow and I am currently a student at PSU pursuing my doctoral degree. I am writing to you to see if you would be willing to participate in my research study. I have attached a flyer to this email with more detail about the study.

The overall goal of this project to is to gain insight into the caregiving experiences of parents of infants and toddlers with autism who are receiving early intervention services. My study will involve two unstructured interviews. I am interested in talking parents with an infant or toddler with autism (between the ages of 24 and 36 months). My study will involve conversations with you about 'what it means to be a parent of a very young child with autism' and your experience with early intervention services.

Most children with autism are not diagnosed until close to age 3 yet research has found that infants display behaviors associated with autism sometime after their first birthday. I am interested in understanding what daily life is like during those early years. I would like my study to give a voice to parents about this unique parenting experience. I believe sharing your personal story is essential to making that happen.

If you have any questions about my study or if you are interested in participating, please contact me: donnab@pdx.edu / 503-756-7006.

Thank you in advance for your consideration, Donna 


\title{
\& Portland State
}

\section{RESEARCH STUDY SEEKING PARENTS WITH A VERY YOUNG CHILD WITH AUTISM}

A Phenomenological Study Lived experiences of Parents of Very Young Children with Autism
Receiving Early Intervention Services

\section{The Study}

Donna Barrow, M.S., a doctoral student at Portland State University is conducting a research study with parents of very young children with autism. The aim of the study is to gain understanding about the day-to-day lived experiences of parenting a very young child with autism who is receiving early intervention services.

\section{Participants}

Looking for participants that meet the following criteria:

- A parent over the age of 18

- A child between 24 and 36 months who is receiving early intervention services for autism

- Family with no other children with disabilities

\section{Why the Study is Being Conducted}

The aim of the study is to understand parent perspectives, and what it means to be a parent of a very young child with autism.

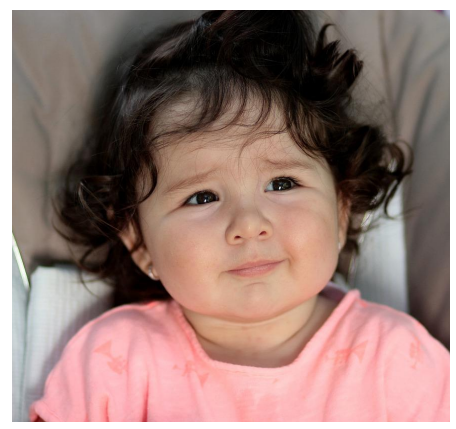

This study is important because while there has been a great deal of research about early intervention models, there has been very little investigations of a parent's point of view. This study is meant to give a voice to parents.

\section{What the Study Involves}

Parents will participate in two interviews ad reflect on parenting their child with autism. Interviews will last 60-90 minutes each. Interviews will take place in the family home or -if preferred-- a location convenient to the parent.

\section{How to Find Out More}

\author{
Contact: \\ Donna Barrow \\ 503-756-7006 \\ donnab@pdx.edu
}




\section{APPENDIX D \\ INITIAL PHONE SCREEN SCRIPT}

Interviewee:

Interview \#:

Time:

Date:

Location:

Thank you for calling to find out more about my research study. This call should take approximately 15 minutes. Before you decide to participate in this study, it is important to understand that Donna Barrow is a doctoral student at Portland State University. She is also an instructor in the Infant Toddler Mental Health program at Portland Sate. She is not affiliated with the Education Service Districts or early intervention services. There are no benefits or drawbacks to participating in this study connected ESDs or EI services. If you are interested in the EI or related programs, a contact will be provided to support you in navigating these programs.

The purpose of this research study to explore parent's experiences with their young children with autism and early intervention programs. The study involves two 60-90 minute interviews. During the first interview I will ask you to select 10 family photographs and reflect on your parenting experiences as we talk about the events and people in the photographs. The second interview will add to our discussion during the first interview and we will also talk about your experiences with early intervention for your child.

As part of the dissertation study, I am asking individuals interested in participating in the study to identify some basic personal screening information. Do you think you would be interested in in participating in this study?

\{If No: $\}$ Thank you very much for calling.

\{If Yes: $\}$ Before enrolling individuals in this study, I need to determine if you are eligible. I would now like to ask you a series of questions about your personal and family demographical information. There is a possibility that some of these questions might make you uncomfortable. If so, please let me know. You don't have to answer any of those questions if you do not want to. You also need to understand that all information I receive from you by phone, including your name and any other identifying information, will be strictly confidential and will be kept locked in a file cabinet in my office. The purpose of these questions is only to determine if you are eligible for the larger study. Remember your participation is voluntary; you do not have to complete these questions. Do I have your permission to ask you these questions?

\{If No: $\}$ Thank you very much for calling.

\{If Yes: $\}$ I have several of personal demographic questions to ask you. 
- Are you at least 18 years of age?

- Are you the parent with the primary caregiving responsibilities for your child?

- Do you have a young child between 24 and 36 months receiving EI services for ASD?

- Are there any other children in the household who are receiving services for disabilities?

Thank you. Additionally, I have a series of questions to ask you.

- Can you provide 10 family photographs of your choice that include the child. We will talk about the pictures during the first interview? These photographs will be used as part of the research study and any publications resulting from the study. Are you comfortable with identifiable information being used as part of the study? You may rescind your decision to consent to the use of family photographs at any time.

- Are you available for two 60-90 minute interviews?

- Are you comfortable with being audio during the interviews?

Once participants have met the study criteria and agreed to participate I will schedule the first interview session to take place in within one week. As part of the study, I will offer to meet participants in their home or a location convenient to them. I will ask them to bring 10 photographs of their family to the first interview. The pictures should include an image of their child but no other parameters for photo selection will be imposed.

\{If No to one or more questions: $\}$ Thank you very much for calling. I sincerely appreciate the time you took to talk with me today. Would you like phone number or website address to obtain additional information about organizations and services for parents of children with autism? (I will offer information using resource sheet). \{If Yes:\} Thank you for answering the questions. You are eligible to participate in the interview study in exploring parenting a young child with autism and their early intervention services.

This interview is designed in two parts (two interviews). The first interview format will include an introduction and a review of the Informed Consent document, telling you about the study and laying out guidelines of what to expect. During the first we will talk about family photographs and your experiences as a parent. Using questions I will share with you, you will have the opportunity to reflect on family life and share experiences about your perspective, thoughts, and feelings about "what it means to be a parent of a young child with autism." The purpose of talking about photographs in this research project is to support you in telling your story of your experiences as a parent. The photographs are a tool for telling your story. There are no right or wrong answers. The second part of the study is a second interview. I will scan the photographs you share with me at our first meeting. The second interview will involve reviewing my notes from the first interview and a conversation about parenting and early intervention services using prompts that I will share with you before we start the interview. 
Are you interested in participant in the study?

\{If No: \} Thank you very much for calling. I sincerely appreciate the time you took to talk with me today. Would you like phone number or website address to obtain additional information about autism services for parents in Oregon?

\{If Yes: \} The study is conducted during the months September 2016 through February 2017 at your home or an agreed upon location that is convenient to you. [schedule interview]

Thank you for agreeing to participate in this study. I will contact you within 48-hours with the times and locations of you interview. My contact information is Donna Barrow donnab@pdx.edu or you can call me at 503-756-7006.

\section{Phone Script Notes:}

Age of child

Autism Eligibility \& EI services $\mathbf{Y}$ or $\mathbf{N}$

Age of parent

Primary caregiver $\mathbf{Y}$ or $\mathbf{N}$

Able to collect 10 photographs with child? $\mathbf{Y}$ or $\mathbf{N}$ 
APPENDIX E

RESOURCE LIST

\section{GENERAL SUPPORT TO FAMILIES IN OREGON}

211 info:

For people living in Oregon and Southwest Washington seeking information on

affordable housing, healthcare, food assistance, and clinics. Access on-line or by phone by dialing 211

\section{OREGON EDUCATION SERVICE DISTRICTS}

$$
\begin{aligned}
& \text { Service Area 1 } \\
& \text { Baker County -- 800-927-5847 } \\
& \text { Grant County -- 800-927-5847 } \\
& \text { Malheur County -- 541-372-2214 } \\
& \text { Morrow County -- 800-927-5847 } \\
& \text { Umatilla County -- 800-927-5847 } \\
& \text { Union County -- 800-927-5847 } \\
& \text { Wallowa County -- 800-927-5847 } \\
& \text { Service Area 2 } \\
& \text { Crook County -- 541-693-5630 } \\
& \text { Deschutes County -- 541-312-1195 } \\
& \text { Gilliam County --541-565-3600 } \\
& \text { Harney County -- 541-573-6461 } \\
& \text { Jefferson County -- 541-693-5740 } \\
& \text { Sherman County -- 541-565-3600 } \\
& \text { Warm Springs -- 541-553-3241 } \\
& \text { Wheeler County -- 541-565-3600 } \\
& \text { Service Area 3 } \\
& \text { Douglas County -- 541-440-4794 } \\
& \text { Jackson County -- 541-494-7800 } \\
& \text { Josephine County -- 541-956-2059 } \\
& \text { Klamath County -- 541-883-4748 } \\
& \text { Lake County -- 541-947-3371 }
\end{aligned}
$$

\author{
Service Area 4 \\ Toll Free -- 877-589-9751 \\ Benton County -- 541-753-1202 x106 \\ Coos County -- 541-269-4524 \\ Curry County -- 541-269-4524 \\ Lincoln County -- 541-574-2240 x100 \\ Linn County -- 541-753-1202 x106

\section{Service Area 5} \\ Toll Free -- 888-560-4666 \\ Marion County -- 503-385-4714 \\ Polk County -- 503-435-5918 \\ Yamhill County -- 503-435-5918

\section{Service Area 6} \\ Multnomah County -- 503-261-5535 \\ Hood River County -- 541-386-4919 \\ Wasco County -- 541-296-1478

\section{Service Area 7} \\ Lane County -- 800-925-8694 \\ 541-346-2578

\section{Service Area 8} \\ Clatsop County -- 503-338-3368 \\ Columbia County -- 503-366-4141 \\ Tillamook County -- 503-842-8423 \\ Washington County \\ English -- 503-614-1446 \\ Spanish -- 503-614-1263

\section{Service Area 9} \\ Clackamas County -- 503-675-4097
}




\title{
HEALTH AND COUNSELING SERVICES FOR FAMILIES WITH CHILDREN WITH SPECIAL NEEDS
}

\author{
Albertina Kerr's Children's Developmental Health Services Center (formerly: Artz \\ Center) \\ 1675 SW Marlow Rd. Suite 200 \\ Portland, OR 97225 \\ 503-228-6479 \\ website: www.AlbertinaKerr.org \\ Autism Treatment and Research Program; Speech and Language Therapy \\ Building Bridges \\ 4724 SW Macadam Ave \\ Portland \\ 503 235-3122 \\ Email: beth@bridgespdx.com \\ Website: bridgespdx.com \\ Our multi-disciplinary team offers complete developmental assessment and medical \\ diagnosis for children up to age 5 years old. Our clinic provides ABA services and \\ biomedical treatment for children with autism.

\section{Children's Program} \\ 7707 SW Capitol Hwy \\ Portland, OR 97219 \\ 503-452-8002, press 2 for information \\ email: info@childrensprogram.com \\ website: www.thechildrensprogram.com \\ "A team of experienced clinicians who provide diagnostic, therapeutic and educational \\ services to children, adolescents, and families."

\section{OHSU at Portland - Autism Program, Child Development \& Rehabilitation Center} \\ (CDRC) \\ 707 SW Gaines Rd \\ Portland, OR 97210 \\ 503-346-0640 \\ 877-346-0640 \\ Diagnosis, monitoring and consultation \\ Providence Neurodevelopment Center for Children (PNCC) \\ 830 N.E. 47th Ave \\ Portland, OR 97213 \\ 503-215-2233 \\ website: www.Providence.org/ChildCenter
}




\section{OREGON AUTISM ORGANIZATIONS - ONLINE}

\section{Portland Oregon Autism Resources - Facebook Group}

A place for autistics, parents, therapists, teachers and caregivers to find information and to advocate for disability rights along with acceptance.

WEBSITE: https://www.facebook.com/Portland-Oregon-Autism-Resource-Guide$\underline{104080276308678 / h o m e}$

\section{Autism Society of Oregon}

Local chapter of Autism Society website provides information about autism, advocacy and attorney list, groups and events in all regions of Oregon, and other resources related to autism for individuals and families.

WEBSITE: http://autismsocietyoregon.org/

\section{Autism Oregon: Connecting Parents and Information}

Website providing lists of providers, support groups, resources and information for parents with children with autism in Oregon.

WEBSITE: http://autismoregon.org/pages/about

\section{NATIONAL AUTISM ORGANIZATION - ONLINE}

\section{UC San Diego}

Information on Autism and typical toddler development, early warning signs, and autism resources:

WEBSITE: https://autism-center.ucsd.edu/autisminformation/Pages/development.aspx

\section{Autism Society - National}

Website with resources for people with autism. Includes advocacy, information, conferences, and research.

WEBSITE: http://www.autism-society.org/ 


\section{APPENDIX F \\ FIRST INTERVIEW SCRIPT \& PROTOCOL}

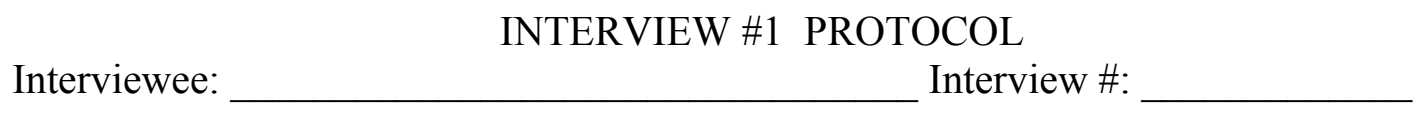

Time: Date: Location:

\section{Introduction to Interview and Photo-Elicitation Activity}

My name is Donna Barrow and I am a doctoral student at Portland State University in the Graduate School of Education and am an instructor for the Infant Toddler Mental Health Program at Portland State University. It is good to meet you in person. Here is my contact information if you need to get ahold of me during the process. My contact information is

Donna Barrow donnab@pdx.edu or you can call me at 503-756-7006. Thank you for your interest participating in the first interview for the research study. We will spend 60-90 minutes together today. We will schedule a second, follow-up interview at the end of this meeting. The purpose of this research study is gain understanding of what having an infant or toddler with autism means from a parent's perspective. This study will explore your day to day experiences with your child and early intervention services for autism. Today's is the first of two interviews. Today's interview will be guided by the family photographs you gathered in preparation for today's meeting. Using the photographs, you will reflect on parenting and experiences with your child. I will ask you questions about the photographs and events depicted in order to get to know what your experiences are like.

I would like to start our time together by reviewing the Informed Consent Document. An informed consent document provides you with information as to what will happen during the interviews, and will provide information about what you can expect during our time together, and speak to the collection and use of data. I brought a copy for you to keep for your records today. At any point that you feel uncomfortable, then please feel free to let me know. If you wish to end our interview at any time, please feel free to do so.

What questions do you have?

Let us review the informed consent form. Review Form.

Do you have any questions?

Thank you for participating in this study.

\section{Introduction to Interviews with Photographs}

Researchers use photographs during interviews when they would like to gather information that cannot be captured by questions alone. Your family photographs offer stories of your experiences The purpose of using photographs is to support you in telling your story of your experiences with your child. There are no expectations about the right way to answer the questions. There is no right way. The photographs are tools for telling 
your story. Your personal story can be defined in may ways...what it means to be a parent to you.... special moment... stories about family life...how your child has changed your family. An interview that uses photographs can help you remember those times or help you show me in addition to tell me. You may rescind consent for use of photographs of your family at any point in the research study for any reason.

I have an audio recorder set-up to capture our conversation. Also, during the interview, you might see me take notes from time-to-time, which is just to help me remember when I look at our interview later.

Would it be all right to have the photographs here next to us? I would like to start by taking photos of the artifacts you selected for today's interview. Is that all right with you? Once you are ready to start, let me know.

On the table, I have left several pieces of paper, each with a prompt about parenting your child. Take a look at the questions and when you are ready, please select photographs to tell me about.

- Pick a photograph and tell me what's happening in the picture.

- Select one of the photographs and tell me why you brought it to talk about during our interview.

- Select a photograph and tell me about the people in it and their relationship with your child.

- Select a photograph that you remember well, tell me about that day and what your family was doing.

You can take as much or little time as you need with each photograph and to talk about the picture.

(After 30 minutes or sooner if participants need) Thank you, would you like to take a break?

When you are ready we can continue the interview.

(At the end of the interview). Thank you for sharing your experiences with me, before we finish for today, is there anything else you would like to share? Can we schedule our next meeting for approximately two weeks from now?

Between now and our second interview, I will be reviewing our conversation, my notes, and the photographs. I may have additional questions before we meet, would it be all right if I called or emailed you. At our second interview, we will talk more about your family experiences and early intervention services.

I want to express my appreciation for your participation in this study and sharing your family experiences with me today. You have my contact information and, if I have any questions, may I follow-up with you? 


\title{
APPENDIX G \\ INFORMED CONSENT \& ASSENT FORMS
}

\author{
Study title: \\ A Phenomenological Study Lived experiences of Parents of Very Young Children \\ with Autism Receiving Early Intervention Services \\ Principal investigator: \\ Randall De Pry \\ Graduate School of Education \\ Special Education Dept. \\ PO Box 751 \\ Portland, OR 97207-0751 \\ 503-725-4493 \\ rdepry@pdx.edu \\ Co-Investigator: \\ Donna Barrow \\ Graduate School of Education \\ Special Education Dept. \\ PO Box 751 \\ Portland, OR 97207-0751 \\ 503-756-7006 \\ donnab@pdx.edu
}

\section{Introduction to the study:}

You are being invited to take part in a research study. Before you decide to participate in this study, it is important to understand that Donna Barrow (me) is a doctoral student at Portland State University and not affiliated with the education service districts (ESDs) or early intervention service providers. There are no benefits or drawbacks to participating in this study as it relates to services or programs associated with Portland State University. The purpose of this research study is to explore parents' experiences caring for a young child with autism. The study involves two 60-90 minute interviews. In the first interview, you will be asked to share photographs of your child and family and reflect on experiences with your child and early intervention programs. Approximately one week later, during a second interview, you will sit with the researcher and talk in more detail about your family life and experiences with early intervention.

It is important that you understand why the research is being done and what it will involve. Please take the time to read the following information carefully. Please ask me if there is anything that is not clear or if you need more information.

\section{What will happen during the study?}

The study will require you to agree to an initial phone interview. The initial phone interview will be approximately 15 minutes. You will also participate in two interviews, each ranging from 60-90 minutes. The study will be conducted during the months of November 2016 through February 2017 at your home or an agreed upon location convenient to you in the Portland metro area. The first interview is a reflective process between you and the researcher using family photographs. During the second interview you will be asked about your experiences as parents and with early intervention programs, at times referring to notes and photographs from the first interview. Data will 
be gathered from your photographs and interviews. The data collected includes an audio recording of our conversation, scanned copies of family photographs, interview notes, and research reflections following the interviews.

The audio will be used to transcribe the audio portion of the conversation. The audio recording will be used to check for concepts and themes from the interview. A copy of your interview transcription, photographic data, and final dissertation will be available on request.

At the end of data-analysis (March - April 2017), you will be invited to participate in a group meeting with other participants. During this meeting we will discuss general themes (no personal or family-specific information will be shared by the researcher). However, as part of the meeting you may discuss personal experiences with the group if you wish. The purpose of this meeting is to "member check" or make sure the researcher has accurately interpreted the themes from the data and to give participants an opportunity to add to the interpretation of the results. The meeting will be approximately 1.5 hours. The group meeting will be held at a private location acceptable to all participants. The final group meeting is not required and you may withdraw consent at any time or opt out for any reason.

Prior to the publication of the final dissertation I will ask you if any or all of your family photographs may be included in the published paper. Photographs will be altered (i.e., blurred, darkened, or otherwise digitally altered) to protect your family's identity. The purpose of using the photographs is to enhance the findings of the study. We will discuss each photograph individually and you will have the opportunity to accept or decline the use of photographs in the final published paper. Further, if you accept use of the photographs I will contact you (a) after 48 hours of acceptance to confirm your consent, and (b) prior to the publication to confirm consent. You may decline use of photographs in the final paper for any reason. Once the paper is published photographs may not be removed.

\section{Risks:}

The risks of participating in the data collection for study are minimal. Data collection activities include interviews, talking about photographs, and a universal group member check meeting. These risks are similar to those you experience when you share family experiences with service providers for your child. If the topics challenge you beyond your comfort level, you may choose to decline to answer any questions in the study and you may terminate your involvement at any time if you choose. As the researcher I will check in with you throughout the interview process and will have resources should you have questions or would like support beyond the scope of the study.

If you consent (and your child assents) to use of photographs in the final dissertation, the risk of using photographs in the final published dissertation include risk of invasion of privacy and breech of confidentiality. While every effort will be taken to obscure your identity by digitally altering photographs and allowing you to choose which (if any) are 
acceptable to use, there is still a risk that your family or members may be identified. By signing this form YOU ARE AGREEING TO WAIVE YOUR CONFIDENTIALITY.

\section{Benefits:}

There will be no direct benefit to you from participating in this study beyond the pleasure of sharing your experiences as a parent. However, I hope that the information from this study will bring parent's voices to the field of autism early intervention research.

\section{How is the participant's (your) privacy protected?}

Every effort will be made by the researcher to preserve your confidentiality including the following: Assigning code names and numbers to all participating individuals that will be used on all researcher photographs, notes, and documents:

- Notes, audio, transcriptions, transcribed notes, and any other identifying participant information will be kept in a locked file cabinet in the personal possession of the researcher. When no longer necessary, all materials will be destroyed.

- Information from this research study will be used solely for the purpose of this study and any publications that may result from this study.

- You have the opportunity to obtain a transcribed copy of the interviews and photographic data. Please tell the researcher if a copy of the interview and photos are desired. You may rescind your consent for participation in the study at any time. If you rescind consent all information gathered during your participation will be immediately destroyed.

You should be aware that the researcher is a mandated reporter by Oregon law and confidentiality is not protected if the researcher is legally required to make a report on an incident involving a child.

\section{Contact:}

Should you have any questions about the research or any related matters, please contact co-investigator Donna Barrow at donnab@pdx.edu or at 503-756-7006. If you have any questions about the co-investigator, please contact principle investigator, Dr. Randall De Pry at 503-725-4493 or rdepry@pdx.edu.

\section{Institutional Review Board:}

If you have any questions regarding your rights as a research subject, or if problem arise which you do not feel like you can discuss with the principle investigator, please contact the Institutional Review Board at hsrrc@pdx.edu_or (503) 725-2227.

\section{Voluntary Participation:}


Your participation in this study is voluntary. It is up to you to decide whether or not to take part in this study. If you decide to take part in this study, you will be asked to sign a consent form. If you decide to take part in this study, you are still free to withdraw at any time and without giving a reason. You are free to answer or not answer any question or questions if you choose. This will not affect any relationship you have with the researcher or have any impact on your participation in any programs related to early intervention services.

\section{Unforeseen risk:}

There may be risks that are not anticipated. However, every effort will be made to minimize any risks.

\section{Cost to participate:}

There is no cost for your participation in this study.

\section{Compensation:}

There is no compensation for this study.

\section{PLEASE READ THE FOLLOWING STATEMENT AND SIGN BELOW IF YOU AGREE.}

I have had the chance to ask any questions I have about this study and my questions have been answered. I have read the information in this consent form and I agree to participate. Select One: YES $\mathrm{NO}$

Signature of the participant

\section{Date}

A copy of this consent form has been given to me for my records: (Initial).

Consent for Use of Individual Photographs

\begin{tabular}{|l|l|l|}
\hline Photograph & $\begin{array}{l}\text { Participant Consent (Y or } \\
\text { N) INITIAL (further } \\
\text { alterations of image?) }\end{array}$ & $\begin{array}{l}\text { Child(ren) Assent } \\
\text { (Y or N) INITIAL }\end{array}$ \\
\hline [participant photograph here] & & \\
\hline [participant photograph here] & & \\
\hline
\end{tabular}




\begin{tabular}{|l|l|l|}
\hline Photograph & $\begin{array}{l}\text { Participant Consent (Y or } \\
\text { N) INITIAL (further } \\
\text { alterations of image? }\end{array}$ & $\begin{array}{l}\text { Child(ren) Assent } \\
\text { (Y or N) INITIAL }\end{array}$ \\
\hline [participant photograph here] & & \\
\hline [participant photograph here] & & \\
\hline [participant photograph here] & & \\
& & \\
\hline
\end{tabular}

Consent Confirmed 48 hours after Original Consent (date) Consent Confirmed prior to publication (date 


\section{Child Assent (Age 12 or Under)}

\section{Study title:}

A Phenomenological Study Lived experiences of Parents of Very Young Children with Autism Receiving Early Intervention Services (IRB163940)

Principal investigator:

Randall De Pry

Graduate School of Education

Special Education Dept.

PO Box 751

Portland, OR 97207-0751

503-725-4493

rdepry@pdx.edu
Co-Investigator:

Donna Barrow

Graduate School of Education

Special Education Dept.

PO Box 751

Portland, OR 97207-0751

503-756-7006

donnab@pdx.edu

\section{Child's name}

Your parents (or guardian) have said that it is okay for you to take part in a project about your family and the things you do together. If you choose to do it, you will be asked if it's ok to use your pictures (show each pictures). They will be changed so it will be hard to see what you look like but other people will be able to see pictures of you and your family.

If do not want me to use your pictures just say no-you won't get into any trouble! In fact, if you don't want to do it at all, you don't have to. Just say so. Also, if you have any questions about the research study, just ask me to explain.

If you do want me to use your pictures, please sign your name on the line below. Remember-you can say no at any time. I will call your parent in a few days and make sure you still are ok with using your pictures.

Child's Signature

Date

Child's Printed Name

Parent's Signature

Date

Parent's Printed Name 


\section{APPENDIX H \\ SECOND INTERVIEW SCRIPT \& PROTOCOL}

Interviewee:

Time: Date: Location:

Interview \#:

\section{Semi-Structured Interview Question}

It is nice to see you again. Here is my contact information again if you need to get ahold of me during the process. My contact information is Donna Barrow donnab@pdx.edu or you can call me at 503-756-700

The purpose of this research study is gain understanding of what having an infant or toddler with autism means from a parent's perspective. This study will explore your day to day experiences with your child and early intervention services for autism.

This is the second interview for the study. There will be two parts to this interview. During the first part we will review the notes from our first interview and take a second look at the photographs you shared. You will have an opportunity to correct, clarify, or add to the summaries from the first interview. During the second portion of the interview, I will ask you questions to gain a better, deeper understanding of your experiences parenting your child and also to understand your experiences and perspectives on early intervention services. We will spend 60-90 minutes together today.

I would like to start our time together by giving you a second copy of the informed consent document and give you an opportunity to ask any questions about your participation in the study. At any point that you feel uncomfortable, then please feel free to let me know. If you wish to end our interview at any time, please feel free to do so. You may rescind your permission for use of photographs at anytime during the study for any reason. What questions do you have?

I have a audio recorder set-up to capture our conversation. Also, during the interview, you might see me take notes from time-to-time, which is just to help me remember when I look at our interview later.

Is that all right with you? Once you are ready to start, let me know.

I'm going to show you the photographs we talked about during our first interview (on my computer). Let's look at these for a moment together. I have a few questions: 1. How was the process of talking about family photographs during the first interview for you?

2. Were there other thoughts about the photographs that came up since we last spoke, that we missed in the first interview?

3. Let's look at the photographs again on my computer

- Can you share what made you choose these particular photographs?

- What feelings did recalling the experiences in the photos bring up for you?

4. I'd like to review the transcript summaries I compiled since we last spoke.

- Can you tell me if I captured what you meant when we discussed these topics?

- Do you have any clarifications, corrections?

- Is there any more information you would like to add to the summaries of the conversations from out last interview? 
(After 30 minutes or sooner if participants need) Thank you, would you like to take a break?

When you are ready we can continue the interview.

\section{SECOND INTERVIEW - PART 2}

The second part of this interview will involve another discussion about your experience as the primary caregiver for your child. We'll also talk about early intervention services. On the table, I have left several pieces of paper, each with a prompt about parenting your child or early intervention services. Take a look at the questions and when you are ready, please one of the questions to talk with me about.

You can take as much or little time as you need with each talk about the with me about the question. The prompts will focus on the following three themes:

Elaborations on family events or experiences (e.g., At our first interview we discussed can you tell me more about that experience).

How EI services impacted your family life. (e.g., The last time we spoke you told me a story about when your child was diagnosed with ASD can you tell me more about that event?)

What it means to have a child with ASD. (e.g., You have mentioned several times that parenting a child with ASD is a journey. Can you tell me more about that?)

(At the end of the interview). Thank you for sharing your experiences with me, before we finish for today, is there anything else you would like to share? Can we schedule our next meeting for approximately two weeks from now?

Over the next few weeks I will be reviewing our conversation, my notes, and the photographs. I may have additional questions, would it be all right if I called or emailed you?

In about a month I will contact you and ask you to read over a summary of our interviews and my conclusions. You will have a chance to correct, clarify, or add to this information either by telephone or email.

I want to express my appreciation for your participation in this study and sharing your family experiences with me today. You have my contact information. Feel free to contact me with any questions. 
PARENTING YOUNG CHILDREN WITH AUTISM

APPENDIX I

ARTIFACT CATALOGUE

Artifact:

Date Received:

Number:

Artifact Type:

- Photo

- Other:

Location:

Description of Artifact:

Event or Contact Associated with Artifact:

Presentation of Artifact:

Brief Summary of Artifact: 
APPENDIX J

AUDIT TRAIL AND AUDITOR ATTESTATION

\begin{tabular}{lll}
\hline Dates of & Activity & Data Collected \\
Project & &
\end{tabular}

\begin{tabular}{|c|c|c|}
\hline August 2016 & $\begin{array}{l}\text { Piloted photo elicitation } \\
\text { interviews (non-participant) }\end{array}$ & $\begin{array}{l}\text { Blog articles with } \\
\text { photo elicitation } \\
\text { interviews }\end{array}$ \\
\hline $\begin{array}{l}\text { October to } \\
\text { November } \\
2016\end{array}$ & $\begin{array}{l}\text { Participant Recruitment at } \\
\text { Columbia Regional, NW Regional } \\
\text { ESD, MESD. Autism Society of } \\
\text { Oregon, early childhood } \\
\text { professionals. } \\
\text { Phone \& in person meetings with } \\
\text { district managers \& directors } \\
\text { Emails to early childhood } \\
\text { professionals. } \\
\text { Posting web announcements. }\end{array}$ & $\begin{array}{l}\text { Activities entered into } \\
\text { research log }\end{array}$ \\
\hline $\begin{array}{l}\text { November } \\
2016\end{array}$ & Initial phone screen. & $\begin{array}{l}\text { Completed screening } \\
\text { instrument and notes } \\
\text { Research log }\end{array}$ \\
\hline $\begin{array}{l}\text { November - } \\
\text { December } 2016\end{array}$ & Email confirmation of interviews. & $\begin{array}{l}\text { Response } \\
\text { Bracketing journal }\end{array}$ \\
\hline $\begin{array}{l}\text { December } 2016 \\
\text { - February } \\
2017\end{array}$ & $\begin{array}{l}\text { First interviews using interview } \\
\text { script, participant photographs, } \\
\text { and prompts }\end{array}$ & $\begin{array}{l}\text { Audio recording } \\
\text { Transcripts } \\
\text { Artifacts } \\
\text { Email response } \\
\text { Researcher Log }\end{array}$ \\
\hline $\begin{array}{l}\text { December } 2016 \\
\text { - } \\
\text { January } 2017\end{array}$ & $\begin{array}{l}\text { Debriefing with } 2 \text { nd } \\
\text { phenomenological researcher } \\
\text { Reflective journaling following } \\
\text { first interviews }\end{array}$ & $\begin{array}{l}\text { Bracketing journal } \\
\text { Meeting Notes IA }\end{array}$ \\
\hline January 2017 & $\begin{array}{l}\text { Email confirmation of second } \\
\text { interviews. }\end{array}$ & $\begin{array}{l}\text { Response } \\
\text { Bracketing journal }\end{array}$ \\
\hline
\end{tabular}




\begin{tabular}{|c|c|c|}
\hline $\begin{array}{l}\text { January - } \\
\text { March } 2017\end{array}$ & $\begin{array}{l}\text { Second interviews using interview } \\
\text { script, participant photographs, } \\
\text { and individualized prompts }\end{array}$ & $\begin{array}{l}\text { Audio recording } \\
\text { Transcripts } \\
\text { Artifacts } \\
\text { Email response } \\
\text { Researcher log }\end{array}$ \\
\hline $\begin{array}{l}\text { January - } \\
\text { March } 2017\end{array}$ & $\begin{array}{l}\text { Member Checking (part } 1 \text { of } 2 \text { nd } \\
\text { interview) }\end{array}$ & $\begin{array}{l}\text { Transcripts } \\
\text { Audio Recording }\end{array}$ \\
\hline $\begin{array}{l}\text { February to } \\
\text { May } 2017\end{array}$ & $\begin{array}{l}\text { Debriefing with } 2 \text { nd } \\
\text { phenomenological researcher } \\
\text { Reflective journaling following } \\
\text { second interviews }\end{array}$ & $\begin{array}{l}\text { Meeting Notes IA } \\
\text { Bracketing journa }\end{array}$ \\
\hline May 2017 & Universal member check meeting & $\begin{array}{l}\text { Audio } \\
\text { recording } \\
\text { Transcript } \\
\text { Researcher } \\
\text { log } \\
\text { Handout } \\
\text { with } \\
\text { essential } \\
\text { themes }\end{array}$ \\
\hline $\begin{array}{l}\text { May - July } \\
2017\end{array}$ & $\begin{array}{l}\text { Narrative and photographic } \\
\text { analysis, reflective writing. }\end{array}$ & $\begin{array}{l}\text { Artifacts } \\
\text { Researcher } \\
\text { log } \\
\text { Reflection } \\
\text { journal }\end{array}$ \\
\hline August 2017 & $\begin{array}{l}\text { Independent audit of research } \\
\text { activities }\end{array}$ & $\begin{array}{l}\text { Auditor } \\
\text { letter }\end{array}$ \\
\hline
\end{tabular}




\title{
PARENTING YOUNG CHILDREN WITH AUTISM
}

\author{
Auditor Attestation \\ A Phenomenological Investigation of the Lived-Experiences \\ of Parents of Young Children with Autism Receiving Early Intervention \\ Doctoral Dissertation \\ Donna Barrow (Randall L. De Pry, Ph.D.) \\ Audited by Heather Bartos, Ed.D.
}

The audit was conducted using the criteria set forth by the researcher (i.e., a modified criteria from Guba and Lincoln's Naturalistic Inquiry (1985)). The researcher prepared an audit trail and samples of research activities upon which I based my attestation. The researcher and I engaged in discussions about data analysis and findings. The phenomenological method rendered attesting to the credibility / validity of the findings (grounded in the data) unwarranted. The findings of this study are based on the researcher's engagement with the data through phenomenological activities (bracketing, peer debriefing, and analysis). Several phenomenological experts weighed in and confirmed the acceptability of the researcher's data analysis and findings. As an independent auditor I reviewed the audit activities and researcher practices for this doctoral dissertation and found the following:

- The audit trail was clear, systematized, complete and comprehensible.

- The credibility of the study was achieved through implementation of member checking following each interview and a universal member check after completion of data collection to confirm essential themes.

- The process of inquiry attested to the dependability of your research. (e.g., interview protocols and sampling methods). Your methodological decisions were purposeful and relevant.

- Your study was conducted in a professional manner and adds to the research in the field of autism early intervention.

Please feel free to contact me if I can provide additional information about this audit.

Signed: Ahay ler Banter, Ed. D. Date 9-19-20/7 Heather Bartos, Ed.D.

Contact Information: $(503) 469-88 / 4$ 